\title{
CROSSTALK BETWEEN OPTICAL WAVEGUIDES WITH APPLICATIONS TO VISUAL PHOTORECEPTORS
}

\author{
PETER MCINTYRE \\ A thesis submitted for the degree of \\ Doctor of PhiZosophy \\ at the Australian National University \\ Canberra, \\ January, 1976
}




\section{PREFACE}

This dissertation is an account of work carried out in the Department of Applied Mathematics of the Institute of Advanced Studies at the Australian National University between March, 1972 and November, 1973 under the supervision of Dr Allan Snyder. Unless stated otherwise, all work is original.

None of the material contained in this thesis has been submitted to any other institution of learning for any degree.

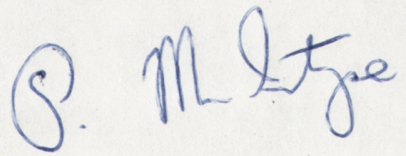

Peter McIntyre 


\section{PUBLICATIONS}

P. McIntyre (1975), "Crosstalk in absorbing optical fibers", J. Opt. Soc. Am. 65, 810.

P. MCIntyre and A.W. Snyder (1973), "Power transfer between optical fibers", J. Opt. Soc. Am. 63, 1518.

P. McIntyre and A.W. Snyder (1974), "Power transfer between non-parallel and tapered optical fịbers", J. Opt. Soc. Am. 64, 285 (1974).

A.W. Snyder and P. McIntyre, "Polarization sensitivity of twisted fused rhabdoms", in Photoreceptor Optics, A.W. Snyder and R. Menzel eds. (Springer-Verlag, Heidelberg, 1975).

A.W. Snyder and P. McIntyre, "Crosstalk between light pipes", J. Opt. Soc. Am. (in press).

P. MCIntyre and A.W. Snyder, "Properties of a twisted anisotropic medium", J. Opt. Soc. Am. (submitted).

A.W. Snyder and P. McIntyre, "Polarization sensitivity and birefringence of twisted photoreceptors", J.Comp.Physiol. (submitted).

P. McIntyre and A.W. Snyder, "Absorption in layered photoreceptors", J. Opt. Soc. Am. (submitted). 


\section{ACKNOWLEDGEMENTS}

It has been a privilege and a pleasure to work under the supervision of Dr Allan Snyder; his insight, enthusiasm and willingness to discuss anything at any time have been an invaluable aid and have also done much to fire my interest in things scientific.

Many thanks are also due to Barry Ninham who has created

a relaxed but stimulating environment which has proved ideal for research. Many other individuals, particularly those in the Vision Group, have contributed through discussions, suggestions, arguments and their uncanny ability to ask awkward questions. Of the others in the department, Derek Chan was always ready, even eager, to challenge everything I said and John Mitchell, the resident mathematician, always available to solve problems when everyone else had failed. I am particularly grateful to Professor Randolf Menzel for the opportunity to work in Darmstadt, West Germany for part of my studies.

Thanks to Pauline Wallace who carried out the mammoth task of transcribing an unreadable manuscript into the finished form. During my studies I was supported by an Australian Postgraduate Research Scholarship and by the Australian National University. 


\section{ABSTRACT}

The greater part of this thesis is concerned with an analysis of the interaction of the electromagnetic fields of neighbouring dielectric waveguides, using the method of coupled-mode theory. Such an interaction leads to an exchange of power or crosstalk between neighbouring waveguides. The concept of crosstalk and some of the necessary definitions are introduced in Chapter 1.

Chapter 2 provides a general introduction to coupled-mode theory, its validity, approximations and solutions of the coupledmode equations. The relationship with the normal-mode approach is examined.

In Chapter 3 we derive a set of degenerate modes for a dielectric waveguide of circular cross-section and use these modes to determine the power transfer between two or more neighbouring waveguides. The validity of the degenerate modes and of coupled-mode theory as applied to waveguides of circular cross-section is also examined.

The theory of Chapter 3 is extended in Chapters 4-6 to determine the crosstalk in several waveguide systems of practical interest. In Chapter 4 we calculate the crosstalk in systems which are not invariant in the longitudinal (z) direction. Two examples analysed in detail are tapered and non-parallel waveguides. Crosstalk between multimode waveguides in which many bound modes can propagate, is investigated in Chapter 5. A comparison of mode and ray theories for multimode waveguides is used to examine some of the difficulties associated with the two theories. Chapter 6 is devoted to crosstalk 
between absorbing waveguides. We show how loss can be included in the coupled-mode theory of crosstalk and determine the power absorbed by each of the waveguides.

In Chapter 7 we use the theory developed in Chapters 3,4 and 6 to analyse crosstalk between visual photoreceptors, in particular between the rhabdomeres of the Dipteran rhabdom. A discussion of modes on photoreceptors and of the implications for and limitations on the operation and evolution of visual systems due to crosstalk is also given.

In Chapter 8 we use coupled-mode theory to examine the propagation of light in a twisted, birefringent, dichroic medium. A simple theory is developed to determine the polarisation properties and absorption of light passing through a slowly twisting medium. The theory is then applied to twisted photoreceptors which have been observed in ants and bees. We determine the effect of the twisting on the absorption properties (polarisation and absolute sensitivities) of the photoreceptors and on the measurement of their birefringence. 
Notes on the text:

(i) References are listed alphabetically at the end of each chapter and are also collected together in the Bibliography:

(ii) The number at the bottom of pages preceding diagrams refers to the next page of text. 
2. COUPLED-MODE THEORY FOR NEIGHBOURING OPTICAL FIBRES

2.1 Introduction

2.2 Derivation of the Coupled-Mode Equations

2.3 Validity and Approximations of the Coupled-Mode Equations

2.3.1 Reduction to two-mode form

2.3.2 Power conservation

2.4 General Solution of Two Coupled Equations

2.5 Normal Modes - Formulation and Discussion

3. POWER TRANSFER BETWEEN CIRCULAR OPTICAL FIBRES

3.1 Outline

3.2. Introduction

3.3 Circular-Waveguide Modes

3.4 Derivation and Validity of the Degenerate $\mathrm{P}_{\mathrm{lm}}$ Waveguide Modes

3.5 Power Transfer between Two Optical Fibres $\quad 51$

3.5.1 Coupling coefficient

3.5.2 Validity of the formulation for two circular waveguides

3.5.3 Simplified theory of power transfer

3.6 Power Transfer in Optical-Fibre Arrays

Appendix: Validity of $\mathrm{P}_{\mathrm{lm}}$ Modes for Coupling between Two Fibres 
4. POWER TRANSFER BETWEEN OPTICAL FIBRES WITH VARIABLE COUPLING

4.1 Outline $\quad \therefore \quad 105$

$4: 2$ Introduction 105

4.3 Formulation $\quad \cdots 7$

4.4 Coupling Coefficients and Power Transfer . 108

4.4.1 Non-parallel fibres 108

4.4.2 Tapered fibres: (linear taper) $\quad 117$

4.5 Discussion 123

References $\quad 125$

5. CROSSTALK BETWEEN MULTIMODE OPTICAL FIBRES

5.1 Outline $\quad 126$

5.2 Introduction 126

5.3 Mathematical Analysis $\quad 128$

5.3.1 Two parallel identical fibres $\quad 128$

5.3.2 Lens excitation 131

5.3.3 Crosstalk power 135

5.3.4 Absorption losses 137

5.3.5 Two parallel fibres with unequal radii 139

5.3 .6 Arrays of fibres 140

5.4 Results $\quad \vdots \quad 140$

5.4.1 Identical fibres 140

5.4.2 Absorption losses 145

5.4 .3 Unequal-radius fibres $\quad 148$

5.4.4 Hexagonal array of fibres 148

5.5 , Discussion . . 150

References $\quad \cdots \quad 160$

6. CROSSTALK BETWEEN ABSORBING OPTICAL FIBRES

6.1 Outline $\quad \cdots \quad 162$

6.2 Introduction $\quad 162$

6.3 Theory $\quad 164$

6.4 Results $\quad 167$

6.5 Excitation and Mode Effects $\quad 176$

References $\quad 179$ 
7. CROSSTALK BETWEEN VISUAL PHOTORECEPTORS

7.1 Outline 180

7.2 Introduction . . . 181

7.3 Structure $\quad \cdots \quad 183$

7.4 Properties of Photoreceptor Modes 188

7.5 Theory of Crosstalk between Photoreceptors 197

7.6 Results 200

7.7 Consequences of Crosstalk $\quad 211$

References $\quad \therefore \quad 219$

8. PROPAGATION OF LIGHT IN TWISTED MEDIA: ABSORPTION AND BIREFRINGENCE IN TWISTED PHOTORECEPTORS

8.1 Outline $\quad 223$

8.2 Introduction 225

8.3 A Coupled-Mode Theory of Light Propagation in a
Twisted Anisotropic Medium

8.4 Properties of Light in a Twisted Medium 231

8.4.1 Polarisation parameters 231

8.4.2 Absorption $\quad 239$

8.5 Theory of Birefringence Measurement $\quad 242$

8.6 Application to Twisted Invertebrate
Photoreceptors

8.6.1 Absorption of light in twisted fused rhabdoms. 248

8.6.2 Absorption of light in twisted fly

8.6.3 Birefringence of twisted rhabdomeres $\quad 267$

References $\quad 270$

BIBLIOGRAPHY 273 


\section{CHAPTER 1}

AN INTRODUCTION

This thesis is concerned with an analysis of certain properties of electromagnetic waves at optical or near-optical frequencies propagating along a dielectric waveguide. The use of such waveguides as a basis for a long-distance communications system has been mooted for a decade or more and owing to recent rapid advances in the fabrication of dielectric waveguides with very low loss (Maurer, 1973), these predictions are likely to be realised in the not-too-distant future (Miller et al, 1973). This has given rise to a spectacular upsurge in both theoretical and experimental research in optical communications, and the field remains a rapidly changing one. We make no attempt here to review recent developments in the field but refer the reader to a number of excellent reviews - Kapany (1967), Kapany and Burke (1972), Marcuse (1972, 1973, 1974), Miller et al (1973), Maurer (1973), Gloge and Marcatili (1973), Gloge (1974), Taylor and Yariv (1974), Snyder (1974), Sandbank (1975) and Clarricoats (1975).

Our analysis is directed to the step-index dielectric waveguide of circular cross-section, although many of the results apply qualitatively to other types of dielectric waveguides. The circular step-index waveguide, also called the step-index optical fibre, is illustrated in Fig. 1.1. The core region of refractive index $n_{1}$ is surrounded by a cladding medium (assumed here to be infinite in extent) of refractive index $n_{2}<n_{1}$. The fact that the core has a higher 
refractive index than the cladding gives the fibre its light-guiding properties. The fibre is assumed to be invariant in the axial $(z)$ direction unless otherwise stated.
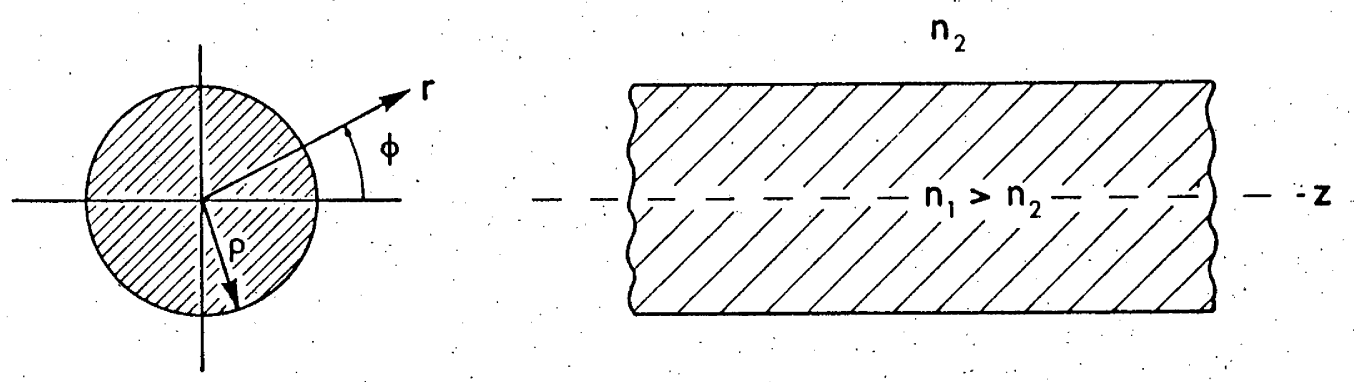

Fig. 1.1

The modal electric and magnetic fields of the optical fibre are solutions of the source-free Maxwell's equations which satisfy the boundary conditions including the radiation condition at infinite distance from the fibre. We assume a time dependence of $e^{-i \omega t}$ and solve the wave equation in the form of the scalar Helmholtz equation to find the components of the time-harmonic fields. The transverse components of the fields are deriveable from the longitudinal (z) components (Adler, 1949), so that we need only find two solutions. The field components in cylinarical geometry are of the form $f(r) e^{i(\beta z-\ell \phi)}$ where $r, \phi$ are the usual cylindrical coordinates and $\beta$ is the (axial) propagation constant of the mode. The functions $f(r)$ are Bessel functions (see Chapter 3):

The modal solutions fall into two categories: a finite set of discrete bound modes and an infinite set of unbound continuous modes. Together they form a complete set of modes for the dielectric waveguide (see for example, Marcuse, 1972). The continuous modes describe the radiation field which radiates from the (core of the) fibre as it 
propagates. An alternative formulation of the radiation field in the region of the fibre in terms of leaky modes is useful when the power inside the fibre is to be determined (Sammut et al, 1975;

Sammut, 1975). The bound modes, on the other hand, propagate along the fibre unchanged except in phase and are therefore present at any distance along the fibre. For the remainder of this thesis we assume that the radiation field has left the fibre and that only the bound modes are present. This approximation is discussed in Snyder (1974) and Sammut (1975). A detailed account of waveguide theory is given in several standard texts such as Stratton (1941), Collin (1961) and in several excellent books specifically on dielectric waveguides, including Kapany and Burke (1972) and Marcuse (1972, 1974).

Some of the properties of the bound modes that are of interest are:

(1) they exist only above a certain cutoff frequency (or below a certain cutoff wavelength), determined for circular optical fibres by the value of $v=\frac{2 \pi \rho}{\lambda}\left(n_{1}^{2}-n_{2}^{2}\right)^{\frac{1}{2}}$ where $\lambda$ is the wavelength in vacuum. When $V$ is below the cutoff value for a mode, the mode is leaky and radiates as it propagates along the fibre (Sammut, 1975).

(2) the propagation constant $\beta$ lies in the range $\mathrm{k}_{2}<\beta<\mathrm{k}_{1}$ where $\mathrm{k}_{1}, \mathrm{k}_{2}$ are the plane-wave propagation constants in the core and cladding media respectively i.e. $\mathrm{k}_{i}=\frac{2 \pi n_{i}}{\lambda}$. Continuous or leaky modes have $\beta<k_{2}, \quad \beta>0$ denotes forward modes (propagating in the $+z$ direction) and $\beta<0$ denotes backward modes.

(3) part of the field of a bound mode propagates along, but outside, the core region in the form of an evanescent field, i.e. the field decays exponentially with distance from the core boundary. 
Because of this property the assumption of an infinite cladding is an approximation to the practical situation of a finite cladding. The infinite cladding assumption is not particularly critical for the behaviour of the bound modes (Clarricoats and Chan, 1973) and this is probably true in many crosstalk problems, although not necessarily so (Marcuse, 1972). We assume an infinite cladding because it leads to simple forms for the crosstalk power; the validity of this approximation in all situations remains to be investigated.

Expressions for the bound-mode fields of an optical fibre are given in Chapter 3.

The greater part of this thesis is concerned with an analysis of a system of two or more neighbouring optical fibres. A phenomenon of such a system is the coupling of modes of the two fibres, which results in power transfer or crosstalk between the fibres. In dielectric waveguides this is due to the presence of the evanescent fields and can occur between two uniform waveguides. In metal waveguides crosstalk does not occur unless some non-uniformity, such as holes spaced along the waveguides, is introduced.

Our analysis is based on coupled-mode theory (CMT) which is introduced in Chapter 2. CMT has been used to predict many effects arising from the non-uniformity of waveguides (Section 2.2) and provides a useful tool for analysing crosstalk between neighbouring fibres. Although the formulation presented here is set up for neighbouring optical fibres some generality is maintained in Chapter 2, so that the theory may be applied to other types of waveguide and non-uniformities. A reading of Chapter 2 is not necessary for an understanding of the remaining chapters. The necessary equations are summarised in 
Section 2.4 and outlined in Section 3.1 .

In Chapter 3 we apply the CMT to optical fibres using a degenerate set of waveguide modes and determine the power transfer between two or more fibres. Chapter 3 also includes a discussion of the validity of CMT and of the degenerate modes as applied to this problem.

The theory of Chapters 2 and 3 is extended in Chapters 4-6 to determine the crosstalk in several fibre systems of practical interest. In Chapter 4 we calculate the crosstalk in systems which are not invariant in the $\mathrm{z}$ direction. Two examples analysed in detail are tapered and non-parallel fibres. Crosstalk between multimode optical fibres in which many bound modes can propagate, is investigated in Chapter 5. A comparison of mode theory with ray theory is used to examine some of the difficulties associated with the ray theory of multimode optical fibres. Chapter 6 is devoted to crosstalk between absorbing optical fibres. We show how loss can be included in the CMT of crosstalk and determine the power absorbed by each of the fibres.

It has been realised for many years that visual photoreceptors may act as dielectric waveguides (Toraldo di Francia, 1948) and this has been confirmed by direct observation (Enoch, 1963). Indeed there has been a great deal of experimental and theoretical research into the effects of confining the absorbing photopigment to a waveguide of diameter of the order of the wavelength of the light it absorbs (see the reviews in Snyder and Menzel, 1975). In many species, including most vertebrates and Dipteran flies, the individual photoreceptors are packed relatively close together (Walls, 1972), thereby allowing for the possibility of crosstalk between receptors. Chapter 7 provides an 
analysis of this crosstalk, and a discussion of the implications and limitations on the operation and the evolution of visual systems.

In Chapter 8 we use CMT to examine the propagation of light in a twisted, birefringent, dichroic medium. A simple theory is developed to determine the polarisation properties and the absorption of light passing through the medium. The theory is applied to twisted photoreceptors which have been observed in ants and bees. We determine the effect of the twisting on the absorption properties (polarisation and absolute sensitivities) of the photoreceptors and on the measurement of their birefringence.

An overall aim of this thesis is to provide a set of simple analytic solutions to a range of problems associated with optical fibres and visual photoreceptors. These simple forms allow the mathematical results to be interpreted in terms of the physical phenomena involved. We make extensive use throughout of the mathematical handbook of Abramowitz and Stegun (1965). References are cited at the end of each chapter and a complete bibliography is given at the end of the thesis. 
REFERENCES

Abramowitz, M. and Stegun, I.A. (1965), Handbook of Mathematical Functions (Dover Publications, New York).

Adler, R.B. (1949), "Properties of guided waves on inhomogeneous cylindrical structures", M.I.T. Technical Report No. 102.

Clarricoats, P.J.B. (1975), "Theory of optical fibre waveguides: a review", in Progress in Optics, E. Wolf, ed. (North Holland Publishing Co., Amsterdam).

Clarricoats, P.J.B. and Chan, K.B. (1973), "Propagation behaviour of cylindrical-dielectric-rod waveguides", Proc. I.E.E. $120,1371$.

Collin, R.E. (1961), Field Theory of Guided Waves (McGraw-Hill, New York).

Enoch, J.M. (1963), "Optical properties of retinal receptors", J. Opt. Soc. Am. 53, 71.

Gloge, D. (1974), "Optical fibers for communication", Appl. Optics . 13,249 .

Gloge, D. and Marcatili, E.A.J. (1973), "Multimode theory of gradedcore fibers", B.S.T.J. $52,1563$.

Kapany, N.S. (1967), Fiber Optics Principles and Applications. (Academic Press, New York).

Kapany, N.S. and Burke, J.J. (1972), Optical Waveguides (Academic Press, New York).

Marcuse, D. (1972), Light Transmission Optics (Van Nostrand Press, New York).

Marcuse, D. (1973), "Optical fibers for communications", Radio. Elec. Engineer 43,655 .

Marcuse, D. (1974), Theory of Dielectric Optical Waveguides (Academic Press, New York).

Maurer, R.D. (1973), "Glass fibers for optical communications", Proc. I.E.E.E. 61, 452 .

Miller, S.E., Marcatili, E.A.J. and Tingye Li (1973), "Research toward optical-fiber transmission systems", Proc. I.E.E.E. 61, 1703.

Sammut, R.A. (1975), "Theory of unbound modes on circular dielectric waveguides", Ph.D. Thesis, Australian National University, Canberra. 
Sammut, R.A., Pask, C. and Snyder, A.w. (1975), "Excitation and power of the unbound modes within a circular dielectric waveguide", PrOC. I.E.E.E. 122, 25.

Sandbank, C.P. (1975), "Fibre optic communications: a survey", Elec. Commun. 50, 20 .

Snyder, A.w. (1974), "Leaky-ray theory of optical waveguides of circular cross-section", Appl. Phys. $\underline{4}, 273$.

Snyder, A.W. and Menzel, R. (eds.) (1975), Photoreceptor Optics (Springer-Verlag, Heidelberg).

Stratton, J.A. (1941), Electromagnetic Theory (McGraw-Hill, New York).

Taylor, H.F. and Yariv, A. (1974), "Guided wave optics"; PrOC. I.E.E.E. 62, 1044.

Toraldo di Francia, G. (1948), "Per una teoria dell'effeto StilesCrawford", Il Nuovo Cimento $\underline{5}, 589$.

Walls, G. (1972), The Vertebrate Eye (Cranbrook Press, Michigan). 
CHAPTER 2

COUPLED-MODE THEORY FOR NEIGHBOURING

OPTICAL FIBRES

\subsection{INTRODUCTION}

The purpose of this chapter is threefold: (1) it provides an introduction to the notation, theory and physical concepts to be used in later chapters; (2) a simple method for the derivation of the coupled-mode equations is outlined following Snyder (1972), and some previous errors are corrected. The general solution to these equations is given; (3) we attempt to introduce the approximations and assumptions involved in the formulation of the coupled-mode equations in a logical and meaningful way, and to determine criteria for the validity of this approach in a general context. In Chapter 3 these are examined for circular dielectric waveguides. This chapter provides the mathematical basis for the theory to be used for later chapters, but need not be read to understand these chapters. The relevant equations are summarised in Section 2.4 and outlined in Section 3.1 .

A common method in electromagnetic theory is the modal approach in which the modes of the system - those fields which propagate unchanged except in phase - are found. This involves solving the wave equation adapted to the particular geometry of the system, and matching solutions at the boundaries to give the modes or eigensolutions. Any field of the system can then be expanded in terms of the modes, with the expansion coefficients determined by certain boundary conditions e.g. initial conditions. This modal-expansion or eigenvector method is physically 
intuitive and straightforward in principle, but modal solutions of the wave equation can only be found for a limited number of systems of relatively simple geometry, including slabs and circular cylinders (Moon and Spencer, 1971).

Coupled-mode theory attempts to preserve the concept of modes for systems in which an exact modal solution is not possible but where the modes of a subsystem are known. These modes, in general, form a complete set so that they can be used to expand the fields of the total system. Because they do not satisfy the boundary conditions of the total system, the modes couple or exchange power as they propagate. A reformulation of Maxwell's equations - the coupled-mode equations determines how the individual mode amplitudes vary as a function of the parameters of the system. The method of coupled modes is most useful when the deviation of the total system from the known subsystem is not too great e.g. small deviations in diameter or refractive index. Although the imperfections may be small they can still produce marked effects, such as total transfer of power from one waveguide to another. There have been many approaches to the problem of mode coupling other than the modal-expansion method used here. Following the early work using CMT by Pierce (1954), Miller (1954), Louisell (1955, 1960), Schelkunoff (1955), and Snitzer (1961), are papers on power transfer between waveguides by Jones (1965) who derives the coupled-mode equations using a Green's function method, Vanclooster and Phariseau (1970, 1970a) who use the reciprocity theorem, Marcuse (1971) with a perturbation approach, Matsuhara and Kumagai (1974) who use variational methods and Arnaud (1974). Several of the methods have been shown to be equivalent (Snyder, 1972; Arnaud, 1974) and all are in substantial agreement (Section 3.5.3). CMT has also been used to treat a variety of other 
problems, including the effect of radius variations and the presence of imperfections and scattering particles on radiation and mode conversion (Marcuse, 1973a, 1974). In many of the problems where the power transfer between modes is small, solutions can also be obtained by other techniques (Snyder, 1974).

\section{Power Transfer between Neighbouring Waveguides}

Coupled-mode theory has particular application to systems in which a large fraction of modal power may be transferred to other modes, as in the case of neighbouring waveguides in which complete transfer of power between waveguides can take place. The well-known phenomenon of frustrated total reflection (Born and Wolf, 1970) is responsible for the light leakage between waveguides, as shown by Kapany and Burke (1972) for slab waveguides. Trapped light rays, corresponding to the slab modes, are incident on the slab boundary at an angle less than the critical angle (measured with respect to the slab boundary) and are therefore totally internally reflected. However in the presence of a neighbouring slab some of the light leaks across the boundary into the neighbouring slab. The total crosstalk power may be found by adding up all rays, taking into account phase and the Goos-Haenchen shift at the slab boundary (see Kapany and Burke, 1972).

The modal explanation of coupling depends on the fact that part of the power of a dielectric waveguide mode propagates along but outside the waveguide in the form of an evanescent field. Part of this evanescent field overlaps the neighbouring waveguide, giving rise to power transfer between the modes of the two waveguides. There is quantitative agreement between the ray and mode approaches for slab waveguides (Kapany and Burke, 1972). 
The ray method because of its simplicity, provides much insight into the phenomenon of coupling between neighbouring slab waveguides, but is difficult to use for other waveguide geometries. In the slab all rays that leak across the waveguide boundary reach the neighbouring slab, whereas, in circular waveguides, only a fraction of these rays are. trapped in the neighbouring guide. The transmission coefficient is therefore difficult to determine (Kapany, 1967). Because of this we use coupled-mode theory rather than the ray approach for circular waveguides, having gained confidence in the knowledge that the two agree for slab waveguides.

In Section 2.2 we outline a derivation of the coupled-mode equations for neighbouring waveguides, followed by a discussion in Section 2.3 on the methods of simplifying the set of equations and on the validity of the simplified equations in representing power flow. The general solution of two coupled equations is given in Section 2.4, together with expressions for the power in each guide and a table summarizing the steps leading to the simplified coupled-mode solution. In Section 2.5 the relationship between the coupled-mode solutions and the normal-mode formulation is examined.

\subsection{DERIVATION OF THE COUPLED-MODE EQUATIONS}

Maxwell's equations for the dielectric media, assuming a time dependence of $\mathrm{e}^{-i \omega t}$ are

$$
\begin{aligned}
& \nabla \times \underline{H}=-i \omega \varepsilon \underline{E} \\
& \nabla \times \underline{E}=i \omega \mu \underline{H}
\end{aligned}
$$

where $\underline{E}, \underline{H}$ are the electric and magnetic field vectors respectively, $\varepsilon$ is the dielectric constant of the medium (may be a tensorial quantity) and $\mu$, the permeability of a vacuum, is assumed to be the same for all media. 
When $\varepsilon$ is a function of the transverse $(x, y)$ coordinates only (Fig. 2.1) the fields of a cylindrical (but not necessarily circular) waveguide, solutions of (2.1), (2.2), are modes of the form (Adler, 1949; Stratton, 1941)

$$
\begin{aligned}
& \underline{E}_{p}=\left(\underline{e}_{p}(x, y)+\underline{\hat{z}}_{p z}(x, y)\right) e^{i \beta_{p} z} \\
& \stackrel{H}{p}_{p}=\left(\underline{h}_{p}(x, y)+\underline{\hat{z}} h_{p z}(x, y)\right) e^{i \beta_{p} z}
\end{aligned}
$$

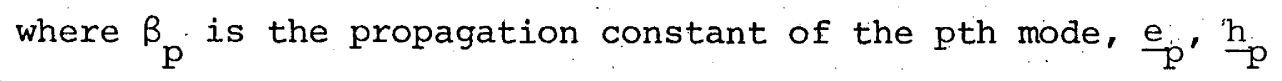
are the transverse modal electric and magnetic fields, and $e_{p z}{ }^{\prime} h_{p z}$ the longitudinal fields. $\hat{z}$ is a unit vector in the $z$ direction and $p$ is non-zero with $p>0(<0)$ representing modes propagating in the $+z(-z)$ direction. The expressions for forward and backward modes are related by

$$
\begin{aligned}
& \underline{e}_{-p}=\frac{e}{p} \\
& \underline{h}_{-p}=-h_{p} \\
& e_{-p z}=-e_{p z} \\
& h_{-p z}=h_{p z} \\
& \beta_{-p}=-\beta_{p}
\end{aligned}
$$

Expressions (2.5) - (2.9) are found by making the transformation $z \rightarrow-z$ in Maxwell's equations.

The modal fields are normalised by

$$
\int_{A_{\infty}} e_{p} \times \underline{h}_{q}^{*} \cdot \hat{z} d A=\gamma_{q} \delta_{p q}
$$

where * denotes complex conjugate, $A_{b_{0}}$ is the infinite cross-sectional area, $\delta_{p q}$ is the Kronecker delta and $\gamma_{q}=1(-1)$ for $q>0(<0)$. 

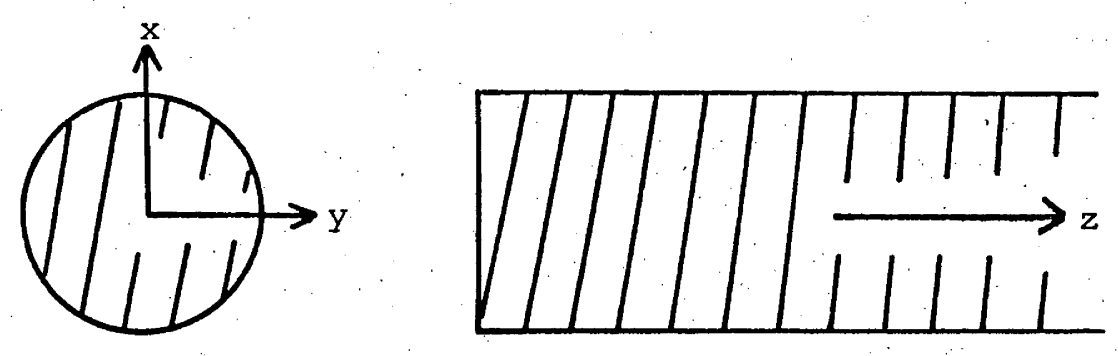

$\underline{E}, \underline{\mathrm{H}}, \varepsilon(\mathrm{x}, \mathrm{Y})$
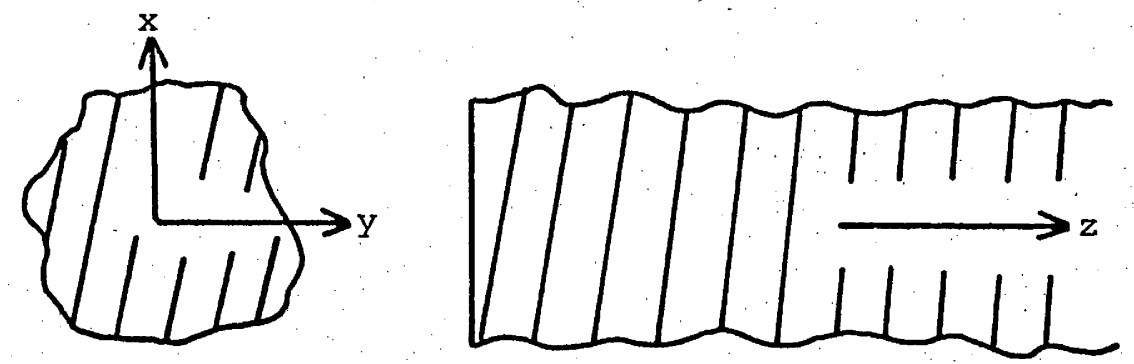

$\widetilde{\underline{E}} \widetilde{\tilde{H}}, \widetilde{\varepsilon}(x, y, z)$

Fig. 2.1

Corresponding expressions for the continuous modes are obtained by making $\mathrm{p}$ a continuous variable and changing $\delta_{\mathrm{pq}}$ to a Dirac delta function (Snyder, 1971). In using the conjugate form of the fields in (2.10), we have assumed the media are lossless. An equivalent formulation to the one presented here, but including loss, is found by using fields in which $w \rightarrow-\omega, \beta \rightarrow-\beta$ instead of the conjugate fields (Marcuse, 1971; Arnaud, 1974). Alternatively loss can be accounted for by the coupledmode formulation (Snyder, 1972 and Chapter 6). With these definitions we now derive the coupled-mode equations following snyder (1972).

$$
\text { Let } \widetilde{\mathrm{E}}, \widetilde{\mathrm{H}}, \widetilde{\varepsilon} \text { be the field vectors and dielectric constant of }
$$
the imperfect or perturbed waveguide (Fig. 2.1), related by (2.1) and (2.2); let $\underline{E}, \underline{H}, \underline{\varepsilon}$ be the corresponding quantities of the ideal waveguide which we take to be uniform and lossless. Define 


$$
\underline{E}=\underline{E} \times \underline{\tilde{H}}^{\star}+\widetilde{E}^{\star} \times \underline{H} .
$$

Application of the divergence theorem to $\underline{F}$ leads to

$$
\int_{A} \nabla \cdot \underline{F}=\frac{\partial}{\partial z} \int_{A} \underline{F} \cdot \underline{\underline{z}} d A+\oint_{L_{A}} \underline{F} \cdot \underline{\hat{n}} d l
$$

where $A$ is an area in the transverse $(x, y)$ plane, $L_{A}$ is a contour bounding this area, and $\underline{\hat{n}}$ is an outward normal unit vector from $L_{A}$. Snyder (1969) discusses the applicability of (2.12) to discontinuous $\underline{F}$ as in, for example, step-index optical fibres.

From (2.11) and a standard vector identity, we have

$$
\nabla \cdot \underline{F}=-i \omega\left(\widetilde{\varepsilon}^{*}-E\right) \underline{E} \cdot \underline{\tilde{E}}^{*}
$$

with

$$
\underline{F}=\underline{F}_{p} e^{i \beta_{p} z}
$$

and $(2.3),(2.4),(2.12),(2.13)$, we find

$$
\left(\frac{\partial}{\partial z}+i \beta_{p}\right) \int_{A_{\infty}} F_{p} \cdot \underline{\hat{z}} d A=-i \omega \int_{A_{\infty}}\left(\widetilde{\varepsilon}^{*}-\varepsilon\right)\left(\underline{e}_{p}+\underline{\hat{z}} e_{p z}\right) \cdot \widetilde{\underline{E}}^{*} d A
$$

The line integral vanishes at infinity because the discrete modes decay exponentially at large distances from the cylinder and the continuous modes vanish due to the limit-point-at-infinity property of the transverse wave equation (Snyder, 1971).

The transverse parts of $\underline{\widetilde{E}}, \underline{\widetilde{H}}$ can be expanded in terms of the complete set of transverse modal fields of the ideal waveguide, giving

$$
\begin{aligned}
& \widetilde{E}_{t}^{*}=\sum_{p} a_{p}(z){\underset{p}{p}}_{p}^{*}(x, y) \\
& \widetilde{H}_{t}^{*}=\sum_{p} a_{p}(z){ }_{p}^{h_{p}^{*}}(x, y)
\end{aligned}
$$

where the summation is understood to extend to an integral over the 
continuous modes. Substitution of (2.16), (2.17) into (2.15) and use of (2.10) gives the general form of the coupled-mode equations

$$
\frac{d a_{p}}{d z}+i \beta_{p} a_{p}=\frac{-i \omega}{2} \gamma_{p} \int_{A_{\infty}}\left(\widetilde{\varepsilon}^{*}-\varepsilon\right)\left(e_{p}+\underline{\hat{z}} e_{p z}\right) \cdot \underline{E}^{*} d A
$$

where $\gamma_{p}$ is defined after $(2.10)$.

The infinite set of equations (2.18). (including the continuous modes) is a reformulation of Maxwell's equations, and as such is exact. In practice, solutions of the equations are obtained by perturbation methods, so that the method is most useful when the fields of the imperfect or perturbed waveguide are not greatly different from those of the ideal waveguide. If this is not the case, the modal expansions (2.16), (2.17) converge slowly.

The $\widetilde{E}^{*}$ in the right-hand side of $(2.18)$ is to be expanded in terms of the fields best suited to the perturbation region (i.e. where the imperfect waveguide differs from the ideal waveguide). These fields may be the modes of the ideal waveguide as in the case of slightly lossy or irregular waveguides, or the modes of a neighbouring waveguide (Snyder, 1972). The expansion of $\underline{E}^{*}$ in terms of the appropriate modes (denoted $e_{q}, e_{q z}$ ) is of the form

$$
\underline{\underline{E}}^{*}=\sum_{q} a_{q^{\prime}}(z)\left[e_{q}^{*}+\underline{\underline{z}} \frac{\varepsilon}{\widetilde{\varepsilon}^{*}} e_{q z}^{*}\right] \text {, }
$$

which when substituted into $(2.18)$ gives

$$
\frac{d a p}{d z}+i \beta_{p} a_{p}=i \sum_{q} C_{p q} a_{q}
$$

where

$$
c_{p q}=-\frac{\gamma_{p} \omega}{2} \int_{A_{\infty}}\left(\widetilde{\varepsilon}^{*}-\varepsilon\right)\left[\frac{e}{p} \cdot e_{q}^{*}+\frac{\varepsilon}{\widetilde{\varepsilon}^{*}} e_{p z} e_{q z}^{*}\right] d A
$$


In terms of the forward mode quantities we can write, using $(2.5)-(2.8)$

$$
C_{p q}=-\frac{\omega}{2} \int_{A_{\infty}}\left(\widetilde{\varepsilon}^{\star}-\varepsilon\right)\left[\gamma_{p}-\frac{e}{p} \cdot e_{-q}^{*}+\frac{\varepsilon}{\widetilde{\varepsilon}^{*}} \gamma_{q} e_{p z} e_{q z}^{*}\right] d A
$$

Equations (2.20) and (2.21) or (2.22) can, in principle, be used to find the effect of any imperfection or perturbation on propagation in a waveguide. See Marcuse (1973a) for examples.

When applied to a system of two neighbouring uniform waveguides, the modes $e_{p}, e_{q}$ are the modes of the individual waveguides in isolation. If mode $\mathrm{p}$ is a forward mode of waveguide 1 and mode $\mathrm{q}$ of waveguide 2 , the coupling coefficient is of the form

$$
C_{p q}^{12}=-\frac{\omega}{2} \int_{A_{2}}\left(\varepsilon_{2}^{*}-\varepsilon\right)\left[\gamma_{p} e_{p}^{1} \cdot e_{p}^{2 *}+\frac{\varepsilon}{\varepsilon_{2}^{*}} \gamma_{q} e_{p z}^{1} e_{q z}^{2 *}\right]
$$

where $A_{2}$ is the cross-sectional area of waveguide $2 ; \varepsilon_{2}$ is its dielectric constant and $\varepsilon$ is now the dielectric constant of the medium surrounding the waveguides.

(2.23) and (2.20) are the starting point for the greater part of the work presented in this thesis (Chapters $3-7$ ).

\subsection{VALIDITY AND APPROXIMATIONS OF THE COUPLED-MODE EQUATIONS}

Equation $(2.20)$ represents an infinite number of coupled differential equations, one for each discrete and continuous mode of the ideal waveguide. Because such a system is not, in general, solvable, we look briefly in this section at the approximations necessary to simplify the set of coupled equations, and at the validity of the equations in representing power flow in the waveguide (s). 
2.3.1 Reduction to Two-Mode Form

Consider first a simple system in which there are only two forward modes and the corresponding backward modes. The system is taken to be lossless so that the propagation constants $\beta_{i}$ are real, and for algebraic simplicity we assume real coupling coefficients, although this is not necessary for our conclusions. Such a system is described by

$$
\begin{aligned}
& \frac{d a_{1}}{d z}+i \beta_{1} a_{1}=i c_{12} a_{2}+i c_{11}^{\dagger} a_{1}^{+}+i c_{12}^{\dagger} a_{2}^{\dagger} \\
& \frac{d a_{2}}{d z}+i \beta_{2} a_{2}=i c_{21} a_{1}+i c_{21}^{\dagger} a_{1}^{\dagger}+i c_{22}^{\dagger} a_{2}^{\dagger} \\
& \frac{d a_{1}^{\dagger}}{d z}-i \beta_{1} a_{1}^{\dagger}=-i c_{11}^{\dagger} a_{1}-i c_{12}^{\dagger} a_{2}-i c_{12} a_{2}^{\dagger} \\
& \frac{d a_{2}^{\dagger}}{d z}-i \beta_{2} a_{2}^{\dagger}=-i c_{21}^{\dagger} a_{1}-i c_{22}^{\dagger} a_{2}-i c_{21} a_{1}^{\dagger},
\end{aligned}
$$

where $a_{i}^{\dagger}$ indicates a backward mode amplitude and $c_{i j}^{\dagger}$ a coupling coefficient between a forward and a backward mode. Self-coupling coefficients are included in the $\beta$ 's.

When

$$
\left|c_{i j}\right| \ll\left|\beta_{i}\right|,\left|\beta_{j}\right|
$$

and

$$
\beta_{1} \approx \beta_{2}
$$

the set of four equations $(2.24)-(2,27)$ decouple into two sets of two equations, one set each for the forward and backward modes; i.e. the eigenvalues found from the four equations are the same as those from the two sets of two equations. As $\beta_{f d}=-\beta_{b d^{\prime}}$ this is consistent with our finding in Section 2.4 that only modes with approximately equal 
$\beta^{\prime}$ 's couple significantly when $(2.28)$ is satisfied.

The equations for the forward modes are

$$
\begin{aligned}
& \frac{d a_{1}}{d z}+i \beta_{1} a_{1}=i c_{12} a_{2} \\
& \frac{d a_{2}}{d z}+i \beta_{2} a_{2}=i c_{21} a_{1}
\end{aligned}
$$

\subsubsection{Power Conservation}

The transverse electric field of the system described by $(2.30),(2.31)$ is given approximately by the sum over the modal fields

$$
\underline{E}_{t} \approx a_{1}^{*} \underline{e}_{1}+a_{2}^{*} \underline{e}_{2}
$$

If $\underline{e}_{1}, \underline{e}_{2}$ are modes of different waveguides, some distortion or overlap of the fields is to be expected. We assume (2.32) and look for conditions under which it provides a consistent description of power flow. The only proper check on the validity of this assumption is provided by solving the exact boundary-value problem for neighbouring waveguides, as has been done numerically by wijngaard (1973) for circular dielectric waveguides. He finds that (2.32) is a reasonable approximation even for touching waveguides (Section 3.5 .3 and Fig. 3.27).

The total power propagating in the $\mathrm{z}$ direction is given by

$$
P(z)=\frac{z}{2} \operatorname{Re} \int_{A_{\infty}} E_{t} \times \underline{H}_{t}^{*} \cdot \underline{\underline{z}} d A,
$$

where $\underline{H}$ is given by a similar expansion to (2.32). For the purposes of this section we assume real fields and also that the magnetic field 
vector is given by $\underline{H}_{t}=\left(\frac{\varepsilon_{1}}{\mu}\right)^{\frac{1}{2}} \hat{}_{\underline{z}} \times \underline{E}_{t}$. This is found to be the case when $\varepsilon_{1} \approx \varepsilon$ (Section 3.3). The power is then given by

$$
P(z) \approx \frac{I}{2}\left\{\left|a_{1}(z)\right|^{2}+\left|a_{2}(z)\right|^{2}\right\}+\operatorname{Re}\left[a_{1}(z) a_{2}^{*}(z)\right] I_{12}
$$

where

$$
I_{12}=\left(\frac{\varepsilon_{1}}{\mu}\right)^{\frac{1}{2}} \int_{A_{\infty}} e_{1} \cdot e_{2} d A
$$

and we have used $(2.10)$. The $\approx$ in $(2.34)$ comes from using (2.32). In most cases $\mathrm{P} \approx \mathrm{P}_{\mathrm{M}^{\prime}}$, where

$$
P_{M}=\frac{1}{2}\left[\left|a_{1}\right|^{2}+\left|a_{2}\right|^{2}\right]
$$

is the sum of the modal powers. We assume a lossless system so that the total power is conserved and is equal to the input power 1 . The small amount of power required for power conservation is given by

$$
P_{D}=1-P_{M}
$$

$\mathrm{P}_{\mathrm{D}}$ consists of two terms: the cross-term in (2.34), arising from the non-orthogonality of the modes $\underline{e}_{1}, \underline{e}_{2}$, and a term which may be thought of as representing the power in the coupling mechanism, by analogy with the stored energy in the spring coupling two pendulums (Louisell, 1960). Multiplication of $(2.30)$ by $a_{1}^{*},(2.30) *$ by $a_{1}$ and similarly with (2.31), and adding gives

$$
\frac{d P}{d z}=\frac{i}{2} \frac{d}{d z}\left[\left|a_{1}\right|^{2}+\left|a_{2}\right|^{2}\right]=\left(C_{12}-C_{21}\right) \operatorname{Im}\left(a_{1} a_{2}^{*}\right)
$$

A similar result is found for the backward modes. Therefore the condition

$$
C_{12}=C_{21}
$$

implies that power as given by the sum of the modal powers (2.36) is 
conserved (by the forward and backward modes separately). An identical calculation with the four coupled equations $(2.24)-(2.27)$ shows that (2.39) is also the condition for power conservation by the sum of forward and backward mode powers as given by the solution of these equations. Therefore (a) (2.39) is a general condition for conservation of the sum of modal powers as given by the coupled-mode formulation (the method leading to $(2.38)$ can be extended to any number of modes), and (b) because $\beta_{1}=\beta_{2}$ (condition $(2.29)$ ) is consistent with $C_{12}=C_{21}$ ' the decoupling of four equations into forward and backward modes remains valid when power considerations are included.

We note at this point that $(2.39)$ is always satisfied for coupling between modes of the same waveguide because of symmetry; $P_{D}$ and the non-conservation of power do not arise in this case. It is only when the modes are of different waveguides, are therefore not orthogonal and (2.32) is an approximation, that we need examine the question of power conservation. The following discussion applies to this case.

Substitution of the solutions for $a_{1}, a_{2}$ given in Section 2.5, into (2.37) leads to

$$
\begin{aligned}
& P_{D}=\left(T+2 X I_{12}\right) F \sin ^{2} \beta_{b} z \\
& T=\frac{C_{12}-C_{21}}{C_{12}},
\end{aligned}
$$

where $F, x, B_{b}$ are defined by $(2.51),(2.50)$ and (2.53) respectively. The quantity $\mathrm{T}$ represents the power in the coupling mechanism and the second term in (2.40) arises from the cross-term.

When $\beta_{1}=\beta_{2}, T$ and $\mathrm{x}$ and therefore $\mathrm{P}_{\mathrm{D}}$ are zero. In this case the only approximations in using the two-equation form and (2.36) for 
the power are $|C| \ll|\beta|$ and the assumptions of (2.32). An approximate calculation of $I_{12}$ gives

$$
2 \times I_{12} \approx \frac{4 \Delta \beta}{\beta \delta}
$$

and the coupling coefficients do not vary greatly with $\Delta \beta$ (see Section 3.5 .2 ), so that, provided $F$ falls off rapidly enough with $\Delta \beta$, ${ }_{D}$ remains small for all $z$ whenever coupling is significant. The condition for rapid fall-off of $F$ with $\Delta \beta$ is $|C| \ll|\beta|$. We conclude that this condition is necessary for power given by (2.36) to be approximately conserved, and is therefore a necessary condition for the coupled-mode formulation to be useful in describing power flow in neighbouring waveguides.

The power in the non-excited waveguide is given by $(2.64)$ as

$$
P_{2}(z)=F \sin ^{2} \beta_{b} z
$$

Comparison of $(2.43)$ with $(2.40)$ shows that $\mathrm{P}_{\mathrm{D}}$ may be a significant fraction of $\mathrm{P}_{2}$. However this only occurs when $\mathrm{F}$ is very small and little power transfer takes place. The significance of $\mathrm{P}_{\mathrm{D}}$ for circular dielectric waveguides is investigated quantitatively in Section 3.5.2.

When there are more than two modes propagating, we follow similar reasoning to that above to separate the coupled-mode equations into sets of equations (usually two) describing coupling between modes with similar $\beta^{\prime}$ s. The effect of a third mode $\left(\beta_{3}\right)$ on mode 1 above is of the order $\frac{C_{13}}{\beta_{1}-\beta_{3}}$ (Johnson, 1965), so that with the assumption $\left|c_{i j}\right| \ll\left|\beta_{i}\right|,\left|\beta_{j}\right|$, unless $\beta_{3} \approx \beta_{1}$ the third mode may be neglected. This allows us in most cases to neglect coupling between the discrete 
and continuous modes because $\beta_{\text {cont. }} \ll \beta_{\text {disc. }}^{\prime}$ except when the discrete mode is near cutoff (Snyder, 1971).

The weak coupling assumption does not imply small total coupling but rather small coupling per unit length. Total power transfer is still possible when $|C| \ll|\beta|$.

\subsection{GENERAL SOLUTION OF TWO COUPLED EQUATIONS}

In 2.3 , we show that in most cases the infinite set of coupled-mode equations $(2.20)$ can be reduced to pairs of coupled equations involving modes with nearly equal propagation constants, i.e. equations of the form

$$
\begin{aligned}
& \frac{d a_{1}}{d z}+i \beta_{1}^{\prime} a_{1}=i c_{12} a_{2} \\
& \frac{d a_{2}}{d z}+i \beta_{2}^{\prime} a_{2}=i c_{21} a_{1},
\end{aligned}
$$

where $\beta_{i}^{\prime}=\beta_{i}-C_{i i}, \beta_{1} \approx \beta_{2}$, the $C_{i i}$ are self-coupling coefficients and $\left|c_{i j}\right| \ll\left|\beta_{i}\right|,\left|\beta_{j}\right|$.

With initial conditions $a_{1}(0)=p, a_{2}(0)=q$, the solution to $(2.44),(2.45)$ is given by

$$
\begin{gathered}
a_{1}(z)=e^{-i \beta_{s}^{z}}\left\{p \cos \beta_{b} z+i \sqrt{F}\left(\frac{C_{12}}{\bar{C}} q-x p\right) \sin \beta_{b} z\right\} \\
a_{2}(z)=e^{-i \beta_{s} z}\left\{q \cos \beta_{b} z+i \sqrt{F}\left(\frac{C_{21}}{\bar{C}} p+x q\right) \sin \beta_{b} z\right\},
\end{gathered}
$$

where

$$
\begin{aligned}
& \beta_{S}=\frac{1}{2}\left(\beta_{1}+\beta_{2}^{\prime}\right) \\
& \bar{C}=\left|C_{12} C_{21}\right|^{\frac{1}{2}}
\end{aligned}
$$




$$
\begin{aligned}
& x=\frac{\beta_{1}^{\prime}-\beta_{2}^{\prime}}{2 \bar{C}} \\
& F=\left(\gamma_{c}+x^{2}\right)^{-1} \\
& \gamma_{c}=\operatorname{sign}\left(C_{12} C_{21}\right) \\
& \beta_{b}=\bar{C} / \sqrt{F}
\end{aligned}
$$

When only two modes are considered (and $|C| \ll|\beta|$ ), the transverse fields are given by

$$
\begin{aligned}
& \underline{E}_{t}=a_{1}^{*} \underline{e}_{1}+a_{2}^{*} \underline{e}_{2} \\
& \underline{H}_{t}=a_{1}^{*} \underline{h}_{1}+a_{2}^{*} \underline{h}_{2}
\end{aligned}
$$

where $\underline{e}_{i}, \underline{h}_{i}$ are the transverse modal electric and magnetic fields respectively. The power is given by

$$
\begin{aligned}
P(z) & =\frac{1}{2} \operatorname{Re} \int_{A_{\infty}} E_{t} \times \underline{H}_{t}^{*} \cdot \underline{\underline{z}} d A \\
& \simeq P_{M}+P_{C}
\end{aligned}
$$

where, using (2.10),

$$
\begin{aligned}
P_{M}(z) & =\frac{1}{2}\left\{\left|a_{1}\right|^{2}+\left|a_{2}\right|^{2}\right\} \\
P_{C}(z) & =\frac{1}{2} \operatorname{Re}\left\{a_{1} a_{2}^{*} \int_{A_{\infty}} e_{1} \times h_{2}^{*}=\underline{z} d A+a_{1}^{*} a_{2} \int_{A_{\infty}} e_{2} \times h_{1}^{*} \cdot \hat{z} d A\right\} \\
& =\frac{1}{2} \operatorname{Re}\left\{a_{1} a_{2}^{*} I_{12}+a_{1}^{*} a_{2} I_{21}\right\}
\end{aligned}
$$

The right-hand side of $(2.58)$ defines $I_{12}$. If the modes are the modes of a single waveguide, $P_{C}=0$ because of mode orthogonality. If they are modes of different waveguides as in a two-waveguide system, $\mathrm{P}_{C}$ is generally small compared with $\mathrm{P}_{\mathrm{M}^{\prime}}$ especially when the waveguides 
are not touching (see sections $3.5 .2,2.3$ ). In most of the following work, we take

$$
P(z) \approx \frac{1}{2}\left\{\left|a_{1}\right|^{2}+\left|a_{2}\right|^{2}\right\},
$$

and normalise to unity initial power, so that

$$
\begin{aligned}
& \frac{1}{2}\left\{|p|^{2}+|q|^{2}\right\}=1 \\
& \text { In many cases of interest, the initial power is all }
\end{aligned}
$$

in one mode (mode 1 say), giving

$$
\begin{aligned}
& a_{1}(z)=\sqrt{2} e^{-i \beta_{s} z}\left\{\cos \beta_{b} z-i x \sqrt{F} \sin \beta_{b} z\right\} \\
& a_{2}(z)=i \sqrt{2} e^{-i \beta_{s} z} \frac{C_{21}}{\bar{C}} \sqrt{F} \sin \beta_{b} z
\end{aligned}
$$

and powers, assuming $\beta_{i}^{\prime}$ real

$$
\begin{aligned}
P_{1}(z) & =\frac{1}{2}\left|a_{1}\right|^{2} \\
& =1-F \sin ^{2} \beta_{b} z \\
P_{2}(z) & =\frac{3}{2}\left|a_{2}\right|^{2} \\
& =\left|\frac{C_{21}}{C_{12}}\right| F \sin ^{2} \beta_{b} z
\end{aligned}
$$

Significant power transfer between the modes will only occur when $F \sim 1$ i.e. $x \leqslant 1$, which requires $\beta_{1} \approx \beta_{2}$ because $|C| \ll|\beta|$. Forward-tobackward-mode coupling and coupling between discrete and continuous modes is therefore very small. The term $\left|\frac{C_{21}}{C_{12}}\right|$ in $(2.64)$ is a consequence of the fact that power given by $(2.59)$ is not conserved unless $C_{12}=C_{21}$ (Section 2.3). The coupling coefficients however are not very sensitive to changes in $\Delta \beta$ and the ratio is very close to 1 whenever power transfer is significant (Section 3.5.2) : 
Table 2.1 summarises the steps and assumptions leading to the decoupled, two-mode form of the coupled-mode equations. It is straightforward in principle to solve more than two equations in the same way as two are solved. The solution of $\mathrm{N}$ equations requires the roots of a polynomial of order $\mathrm{N}$, and for $\mathrm{N}>2$ the resultant expressions are much more complicated than those for $\mathrm{N}=2$ found here. 


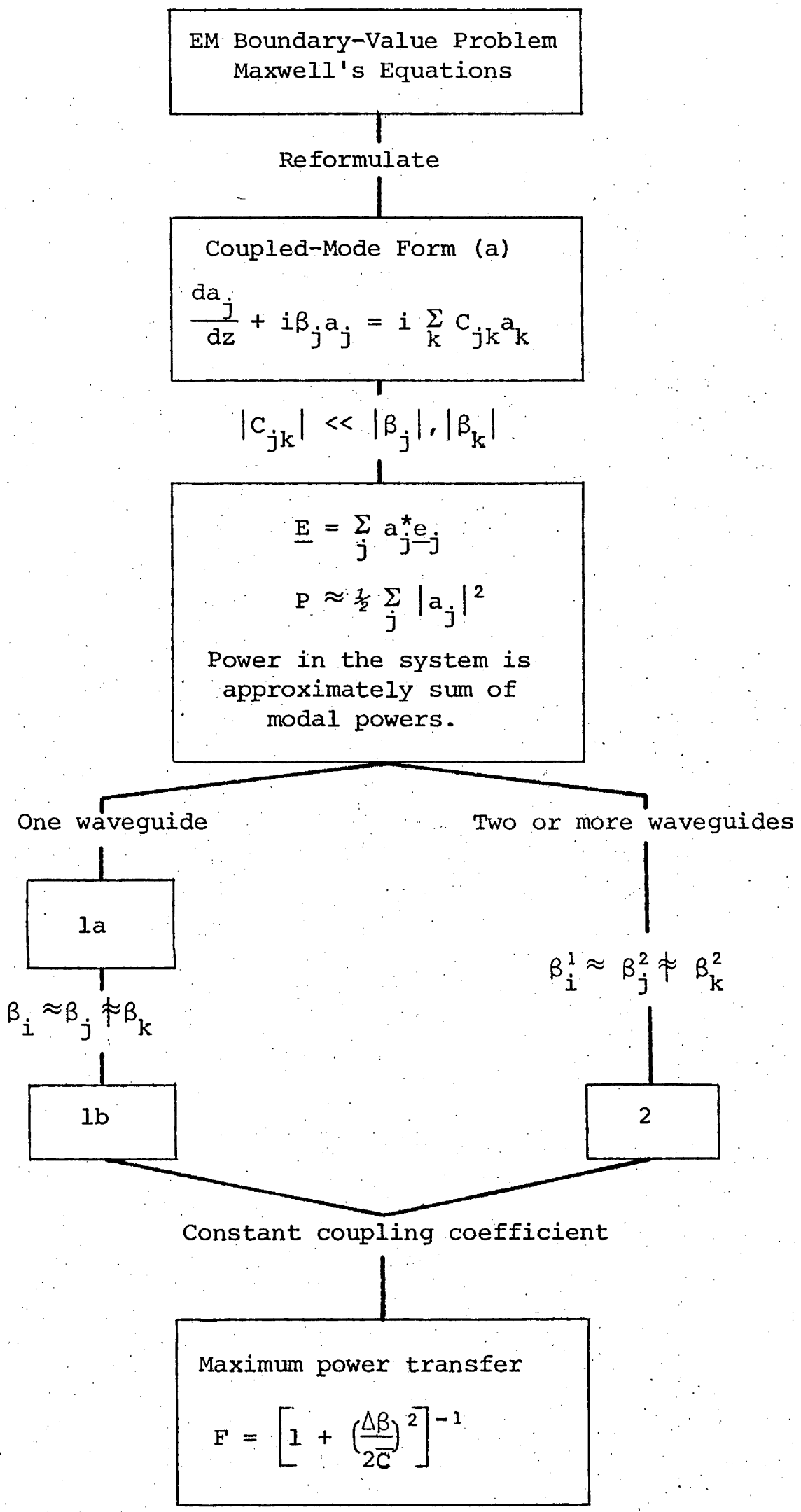

Table 2.1 
Table 2.1 A summary of the steps leading to the decoupled, two-mode equations $(2.44),(2.45)$

(a) The coupled-mode equations are an exact reformulation of Maxwell's equations. The radiation field (continuous modes) is included by extending all sums to integrals over the continuous mode parameters.

(b) The fields of a system can always be expanded in terms of the modes of the ideal waveguide(s) even if the condition $|c|<|\beta|$ is not satisfied, but the field and power expressions are complicated and the coupled-mode formalism is probably not very useful. This condition indicates that it is valid to approximate the total field. as a superposition of the fields of the ideal waveguide(s).

1a. If the system is only one waveguide, the fields at the perturbation e.g. Ioss or irregularity of the waveguide, are expanded in terms of the modes of the ideal waveguide. This gives rise to selfcoupling terms which modify the propagation constants of the modes, and to coupling between modes. On one waveguide, by symmetry, $C_{i j}=C_{j i}$ so that power $(2.59)$ is conserved by forward and backward modes separately (assuming constant $\mathrm{C}_{i j}$ ).

1b. Mode $i$ only couples strongly to mode $j$, giving a twomode form. Self-coupling may be important as in the case of a lossy waveguide. If $N$ B's are approximately equal, $N$ equations must be solved.

2. Waveguide 2 is a perturbation on the fields of waveguide 1. The fields in the perturbation region are therefore expanded in terms of the modes of waveguide 2 . Mode $i$ on waveguide 1 only couples strongly with mode $j$ on waveguide $2 \cdot c_{i j} \approx_{C_{j i}}$ so that power (2.59) is approximately conserved by the forward and backward modes separately. The coupled-mode equations reduce to the two-mode form: 
We have assumed that the coupling coefficients are not functions of $z$. If the waveguide is inhomogeneous in the $z$ direction, this is not the case and forward-to-backward-mode coupling may occur. 


\subsection{NORMAL MODES - FORMULATION AND DISCUSSION}

Coupled-mode theory solves the problem of electromagneticwave propagation in a system using the modes of a simpler subsystem e.g. of individual waveguides in a two-waveguide system. These modes do not satisfy the boundary conditions of the system and therefore couple or exchange power as they propagate. The normal modes on the other hand do satisfy the boundary conditions and therefore propagate unchanged except in phase. Visualising coupling, especially coupling between neighbouring waveguides, in terms of the normal modes is a useful alternative means of relating the theory to the physics of the coupling problem. A third method using ray concepts is outlined in Section 2.1 and analysed in Kapany and Burke (1972).

We consider a system of two parallel waveguides described by (2.44), (2.45). In terms of the individual-waveguide modes $\underline{e}_{1}, \underline{e}_{2}$ the field due to modes 1,2 is given by

$$
\underline{E}(z)=a_{1}^{*}(z) \underline{e}_{1}+a_{2}^{*}(z) \underline{e}_{2}
$$

In terms of the normal modes $\Psi_{1}, \Psi_{2}$, the field is given by an expression of the form

$$
E(z)=A_{1} \Psi_{1} e^{i \lambda_{1} z}+A_{2} \Psi_{2} e^{i \lambda_{2} z}
$$

where $A_{1}, A_{2}$ are independent of $z, \quad \psi_{1}, \psi_{2}, \underline{e}_{1}, \underline{e}_{2}$ are all functions of $x, y$ only. The two normal modes propagate with constant amplitudes but interfere, i.e. beat together, to produce an intensity pattern that varies with $z$. This changing pattern gives the appearance of the individual-waveguide modes exchanging power.

The normal modes can be found from the coupled-mode formulation (Snyder, 1974a; Friedman, 1956) giving 


$$
\begin{aligned}
& \Psi_{1}=\left[\underline{e}_{1}+(\gamma+\eta) \underline{e}_{2}\right] / \sqrt{N_{1}} \\
& \Psi_{2}=\left[\underline{e}_{1}+(\gamma-\eta) \underline{e}_{2}\right] / \sqrt{N_{2}},
\end{aligned}
$$

where $\gamma=\frac{\bar{C} X}{C_{12}}, \eta=\frac{\bar{C} \sqrt{1+X^{2}}}{C_{12}}\left(\gamma_{C}=1\right)$ and the normalisation constants by

$$
\begin{aligned}
N_{1,2} & =1+(\gamma \pm \eta)^{2}+2(\gamma \pm \eta) I_{12} \\
I_{12} & =\left(\frac{\varepsilon_{1}}{\mu}\right)^{\frac{1}{2}} \int_{A_{\infty}} \underline{e}_{1} \cdot \underline{e}_{2} d A
\end{aligned}
$$

We have assumed for simplicity that $\varepsilon_{1}=\varepsilon_{2}$ and $\underline{h}_{i}=\left(\frac{\varepsilon_{1}}{\mu}\right)^{\frac{1}{2}} \underline{\underline{z}} \times \underline{e}_{i}$. The corresponding amplitudes are given by (Louisell, 1960)

$$
\begin{aligned}
& A_{1} e^{i \lambda_{1} z}=\frac{-\sqrt{N_{1}}}{2 \eta}\left[(\gamma-\eta) a_{1}-a_{2}\right] \\
& A_{2} e^{i \lambda_{2} z}=\frac{\sqrt{N_{2}}}{2 \eta}\left[(\gamma+n) a_{1}-a_{2}\right],
\end{aligned}
$$

where $\lambda_{1}, \lambda_{2}$ are the eigenvalues of the coupling matrix $\left(\begin{array}{cc}\beta_{1} & -C_{12} \\ -C_{21} & \beta_{2}\end{array}\right)$ i.e.

$$
\lambda_{1,2}=-\beta_{s} \pm \bar{C} / \sqrt{F}
$$

When $\beta_{1}=\beta_{2}, \gamma=0$ and $n=1$ so that the normal modes interfere to give zero amplitude first in one waveguide and then the other as they propagate. This corresponds to the waveguide modes exchanging power with transfer length $\frac{\pi}{\lambda_{1}-\lambda_{2}}=\frac{\pi}{2 \bar{C}}$, as found before. If $\beta_{1} \neq \beta_{2}$ complete interference cannot take place, resulting in an incomplete exchange of power between the waveguides.

The normal modes found here are only an approximation to the 
true normal modes of the system because we have used the approximate form of the coupled-mode equations to derive them, rather than the infinite set including the continuous modes. This formulation therefore provides no additional information. Wijngaard (1973) solves for the normal modes of a two-waveguide system numerically and finds that the approximate analysis presented here (in either the coupledmode or normal-mode form) provides a good description of the system even for touching waveguides (see Sections 3.5.2, 3.5.3). 


\section{REFERENCES}

Adler, R.B. (1949), "Properties of guided waves on inhomogeneous cylindrical structures", M.I.T. Technical Report No. 102.

Arnaud, J.A. (1974), "Transverse coupling in fiber optics Part I: Coupling between trapped modes", B.S.T.J. 53, 217.

Born, M. and Wolf, E. (1970), Principles of Optics (Pergamon Press, New York, 2nd edition).

Friedman, B. (1956), Principles of Applied Mathematics (Wiley, New York).

Johnson, C.C. (1965), Field and Wave Electrodynamics (McGraw-Hill, New York), p.314.

Jones, A.L. (1965), "Coupling of optical fibers and scattering in fibers", J. Opt. Soc. Am. 55, 261.

Kapany, N.S. (1967), Fiber Optics Principles and Applications (Academic Press, New York).

Kapany, N.S. and Burke, J.J. (1972), Optical Waveguides (Academic Press, New York).

Louisel1, W.H. (1955), "Analysis of the single tapered mode coupler", B.S.T.J. 34, 853 .

Louisell, W.H. (1960), Coupled Mode and Parametric Electronics (Wiley, New York):

Marcuse, D. (1971), "The coupling of degenerate modes in two parallel dielectric waveguides", B.S.T.J. 50. 1791.

Marcuse, D. (1973a), "Coupled mode theory of round optical fibers", B.S.T.J. 52, 817 .

Marcuse, D. (1974), Theory of Dielectric Optical Waveguides (Academic Press, New York).

Matsuhara, M. and Kumagai, N. (1974), "Theory of coupled open transmission lines and its applications", I.E.E.E. Microwave Theory Tech. MTT'-22, 378.

Miller, S.E. (1954), "Coupled wave theory and waveguide applications", B.S.T.J. 33,661 .

Moon, P.H. and Spencer, D.E. (1971), Field Theory Handbook (Springer-Verlag, Berlin). 
Pierce, J.L. (1954), "Coupling of modes of propagation", J. Appl. Phys. 25, 179.

Schelkunoff, S.A. (1955), "Conversion of Maxwell's equations into generalized telegraphist's equations", B.S.T.J. 34, 995.

Snyder, A.W. (1969), "Wave propagation along dielectric structures with application to retinal receptors", Ph.D. Thesis, University of London.

Snyder, A.W." (1971), "Continuous mode spectrum of a circular dielectric rod", I.E.E.E. Microwave Theory.Tech. MTT-19, 720.

Snyder, A.W. (1972), "Coupled-mode theory for optical fibers", J. Opt. Soc. Am. 62, 1267.

Snyder, A.W. (1974), "Leaky-ray theory of optical waveguides of circular cross section", Appl. Phys. ㄴ. 273.

Snyder, A.W. (1974a), "Light absorption in visual photoreceptors", J. Opt. Soc. Am. 64, 216.

Snitzer, E. (1961), in Advances in Quantum Electronics (Columbia University Press, New York), p.219.

Stratton, J.A. (1941), EZectromagnetic Theory (McGraw-Hill, New York).

Vanclooster, R, and Phariseau, P. (1970), "The coupling of two parallel dielectric fibers I. Basic equations", Physica 47, 485.

Vanclooster, R. and Phariseau, P. (1970a), "The coupling of two parallel dielectric fibers II. Characteristics of the coupling in two fibers", Physica 47, 501.

Wijngaard, W. (1973), "Guided normal modes of two parallel circular dielectric rods", J. Opt. Soc. Am. 63, 944. 
CHAPTER 3

POWER TRANSFER BETWEEN CIRCULAR OPTICAL FIBRES

\subsection{OUTLINE}

From Chapter 2, the power propagating along two neighbouring lossless dielectric waveguides when waveguide 1 is initially excited with unit power, is given by

$$
\begin{aligned}
& P_{1}(z)=1-F \sin ^{2} \beta_{b} z \\
& P_{2}(z)=\left|\frac{C_{21}}{C_{12}}\right| F \sin ^{2} \beta_{b} z
\end{aligned}
$$

where $C_{12}, C_{21}$ are the coupling coefficients

$$
c_{12}=-\frac{\omega}{2} \int_{A_{2}}\left(n_{2}^{2}-n^{2}\right)\left[\underline{e}_{1} \cdot \underline{e}_{2}^{*}+\left(\frac{n}{n_{2}}\right)^{2} e_{z}^{1} e_{z}^{2 *}\right] d A
$$

$\mathrm{n}_{2}, \mathrm{n}$ are the refractive indexes of waveguide 2 and the surrounding medium respectively, $\underline{e}_{i}, e_{z}^{i}$ are the transverse and longitudinal modal electric vectors and $A_{2}$ is the cross-section of waveguide 2 . The maximum power transfer $F=\left[1+\frac{(\Delta \beta)^{2}}{4 \mid C_{12} C_{21}}\right]^{-1}$ where $\Delta \beta$ is the difference between the propagation constants of the modes on the two waveguides, and $B_{b}=\left|C_{12} C_{21}\right|^{\frac{7}{2}} / \sqrt{F}$.

In this chapter we calculate values for the coupling coefficients for parallel waveguides of circular cross-section (optical fibres), using a set of degenerate waveguide modes. The validity of these modes as modes and for use in a coupled-mode theory of parallel optical fibres is examined and the validity of the coupled-mode formalism is also 
checked. We obtain expressions for the power transfer between any two modes on optical fibres of arbitrary radius and refractive index. Examples are given and the effect of varying the fibre parameters is examined. As found previously, total power transfer can only take place when the propagation constants of the two modes are equal e.g. like modes on identical fibres.

In the last section we look at power transfer in optical fibre arrays and compare it with power transfer between two fibres. We find that the greater the number of fibres in the array the greater the initial rate of power transfer but that in an array of identical fibres, incomplete power transfer takes place between the central fibre and the surrounding fibres.

The important results of this chapter are presented in Section 3.5.3 in which a general expression (3.56) for the coupling coefficient is given, and in section 3.6 on arrays.

\subsection{INTRODUCTION}

Chapter 2 and the references quoted there, especially Snyder (1972) and Marcuse $(1972,1974)$, provide a general coupledmode theory which may be applied to a variety of problems. However, to be of practical use, the theory requires two things: (1) a knowledge of the modes of the ideal waveguide, in order that the coupling coefficient may be calculated, and (2) use of approximations to reduce the coupled-mode equations to a more easily solvable form. The procedure in (2) has already been indicated in section 2.3. In this chapter, we use the known modes of a circular dielectric waveguide or optical fibre to calculate the power transfer between two or more neighbouring, parallel optical fibres. 
The circular waveguide and the slab waveguide with step refractive-index profiles are two of the geometries frequently used as optical waveguides and, fortunately for the theorist, are two systems for which the electromagnetic modes may be found. They therefore form an ideal basis for acoupled-mode theory of waveguide perturbations. Other promising fibres for communication purposes, such as the gradedindex fibre, require approximation techniques to find the fields (see Clarricoats, 1975 for a review). The slab waveguide in particular, because of its relative simplicity, has been analysed in great detail (review Taylor and Yariv, 1974; Marcuse, 1972; Wilson and Reinhart, 1974). In some cases the normal modes of more complicated systems involving slabs, e.g. two parallel slabs, can be found exactly or to a very good approximation (Marcatili, 1969). This provides a check on the accuracy of the coupled-mode formulation and gives a basis from which we can apply the theory to more complicated geometries. The ray theory of slab coupling is also relatively simple and provides further verification of the theory (Kapany and Burke, 1972). Papers using mode theory for circular optical fibres include Bracey et al (1959), Snitzer (1961, 1963), Jones (1965), Kapany et al (1965), Burke (1967), Vanclooster and Phariseau (1970, 1970a), Marcuse (1971), Snyder (1972) and Wijngaard (1973).

Two problems related to that of crosstalk between bound modes on optical fibres but not considered here are coupling between leaky modes on optical fibres and crosstalk between graded-refractive-index fibres. Both these problems deserve investigation.

In the limit $\delta=1-\left(\frac{n_{2}}{n_{1}}\right)^{2} \ll 1$, Snyder $(1969$, 1969a) has shown that the expressions for the modal fields of a dielectric circular waveguide simplify; we give these simplified modes in 
Section 3.3. The restriction $\delta \ll 1$ is satisfied in most optical communications fibres and visual photoreceptors, and is therefore not greatly restrictive. In fact, even when $\delta \sim 0.6$, the $\delta \ll 1$ approximate results are not greatly in error (Snyder, 1972). Although it is not necessary to assume $\delta<1$ to use coupled-mode theory for circular fibres, we do so here for two reasons: first, because it results in simple expressions for the modes and coupling parameters which can be readily interpreted and related to the physics of the system, and second, because there seems to be no reason to introduce unnecessary complexity into systems for which $\delta \ll 1$ in almost all cases. The reader is referred to Marcuse (1974) or Kapany and Burke (1972) for full modal expressions, and to Marcuse (1971) who analyses mode coupling between circular dielectric waveguides without imposing this restriction.

In Section 3.4 we present a brief derivation and discussion of the degenerate waveguide modes, valid in the limit $\delta \rightarrow 0$, followed by the theory of power transfer between two circular optical fibres in section 3.5. This includes a discussion of the validity of the approximations outlined in Section 2.3 , together with the derivation of expressions for the coupling coefficient. In Section 3.6, we generalise to coupling in fibre arrays and show how symmetries in the array geometry lead to simple expressions for the power transfer. In the Appendix we discuss the validity of using the $\mathrm{P}_{\ell m}$ modes $(\ell>0)$ for coupling between two fibres and relate this to the normal modes of a two-fibre system as calculated by Wijngaard (1973). Coupling coefficients for coupling between $\mathrm{HE}$ and $\mathrm{EH}$ modes and a summary of the approximations are also given.

For the remainder of this thesis we assume all modes to be propagating in the forward $(+z)$ direction. 


\subsection{CIRCULAR-WAVEGUIDE MODES}

The longitudinal ( $z$ ) components of the time-harmonic, modal fields (2.3), (2.4), of a circular, dielectric, step-index waveguide (Fig. 3.1) are solutions of the normalised transverse wave equation (Stratton, 1941; Adler, 1949)

$$
\left\{\rho^{2} \nabla_{t}^{2}+\left(\begin{array}{r}
U_{p}^{2} \\
-w_{p}^{2}
\end{array}\right\} \Phi_{p z}=0 \quad \begin{array}{l}
R>1 \\
R<1
\end{array}\right.
$$

where $U_{p}^{2}=\rho^{2}\left(k_{1}^{2}-\beta_{p}^{2}\right), w_{p}^{2}=\rho^{2}\left(\beta_{p}^{2}-k_{2}^{2}\right), k_{i}=\frac{2 \pi n}{\lambda}, R=r / \rho, \lambda$ is the wavelength in vacuum and $\nabla_{t}^{2}=\nabla^{2}-\frac{\partial^{2}}{\partial z^{2}} \cdot$ Subscript $p$ is the mode label. The transverse components of the field vectors are related to the longitudinal components by equations which, when $\delta=1-\left(\frac{n_{2}}{n_{1}}\right)^{2} \ll 1$, take the form (Snyder, 1969a)
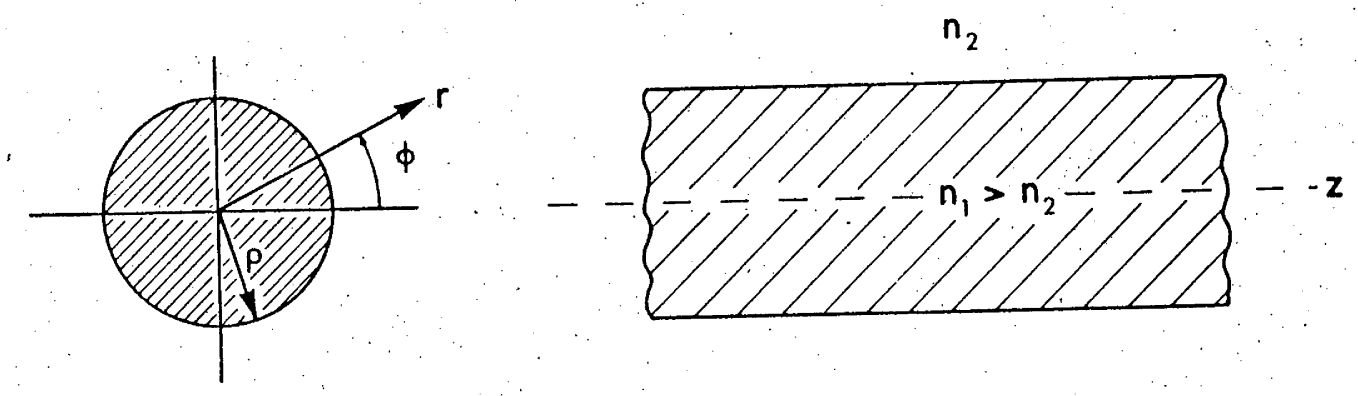

Fig. 3.1 


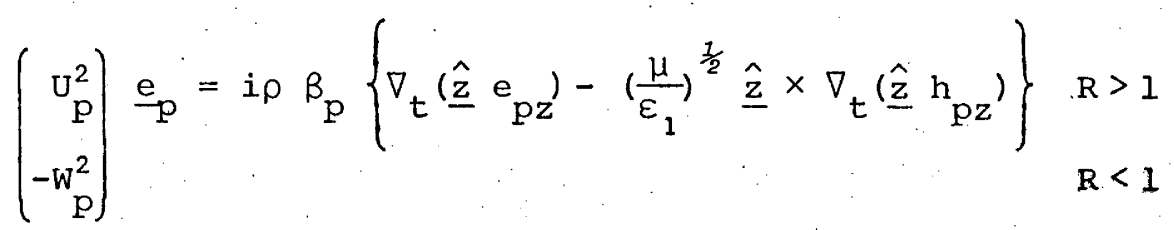

$$
\begin{aligned}
& \underline{h}_{p}=\left(\frac{\varepsilon_{1}}{\mu}\right)^{\frac{1}{2}} \underline{\hat{z}} \times \frac{\mathrm{e}}{\mathrm{p}} ;
\end{aligned}
$$

where $\underline{\hat{z}}$ is a unit vector in the $\mathrm{z}$ direction, $\mu$ is the relative permeability of a vacuum and $\varepsilon_{1}=n_{1}^{2}$. The solutions of the scalar Helmholtz equation (3.1) in cylindrical coordinates, are in terms of Bessel functions in the radial variable $r$ and trigonometric functions in $\phi$ (Stratton, 1941). Application of the boundary conditions at the core-cladding boundary $(R=1)$ and at infinity, gives the solutions as $\mathrm{HE}, \mathrm{EH}, \mathrm{TE}, \mathrm{TM}$ modes, with components

$$
\begin{aligned}
& e_{z}=i \sqrt{\delta} \frac{J_{\ell}(U)}{J_{\ell \mp 1}(U)} \quad\left\{\begin{array}{ll}
\sin \ell \phi \\
\cos \ell \phi
\end{array}\right\} f_{\ell \pm 1}(R) \\
& h_{z}=\mp i \sqrt{\delta}\left(\frac{\varepsilon_{1}}{\mu}\right)^{\frac{1}{2}} \frac{J_{\ell}(U)}{J_{\ell \mp 1}(U)}\left\{\begin{array}{l}
\cos \ell \phi \\
-\sin \ell \phi
\end{array}\right\} f_{\ell \pm 1}(R) \\
& e_{r}= \pm f_{\ell}^{(R)}\left\{\begin{array}{ll}
\sin \ell \phi \\
\cos \ell \phi
\end{array}\right\} \\
& e_{\phi}=f_{\ell}(R)\left\{\begin{aligned}
\cos \ell \phi \\
-\sin \ell \phi
\end{aligned}\right\}
\end{aligned}
$$

where

$$
\begin{aligned}
f_{\ell}(R) & =\frac{J_{\ell \mp 1}(U R)}{J_{\ell \mp 1}(U)} \quad R \leqslant 1 \\
& =\frac{K_{\ell \mp 1}(W R)}{K_{\ell \mp 1}(W)} \quad R \geqslant 1
\end{aligned}
$$

The upper (lower) signs refer to the HE(EH) modes. The TE, TM modes are $\mathrm{EH}_{0 \mathrm{~m}}$ modes.

The transverse eigenvalues $U, W$ of the waveguide are related by the eigenvalue equation

$$
\frac{U_{\ell} J_{\ell}(U)}{J_{\ell \mp 1}(U)}= \pm \frac{W \cdot K_{\ell}(W)}{K_{\ell \mp, 1}(W)}
$$


and by

$$
\mathrm{v}^{2}=\mathrm{u}^{2}+\mathrm{w}^{2}
$$

where

$$
\begin{aligned}
\mathrm{V} & =\frac{2 \pi \rho}{\lambda} \mathrm{n}_{1} \sqrt{\delta} \\
& =\rho \omega \mathrm{n}_{1}(\mu \delta)^{\frac{1}{2}},
\end{aligned}
$$

is a characteristic parameter of the waveguide. $\delta \ll 1$ implies $\beta \approx \mathrm{k}_{2} \approx \mathrm{k}_{1}=\frac{\mathrm{v}}{\rho \sqrt{\delta}}$

The modes are labelled by two positive integer indexes $l, m$ where $U_{\ell m}$ is the $m$ th root of $(3.10)$, lis related to the angular variations in the fields and $m$ to the radial variations. The general mode index $\mathrm{p}$ is understood to range over all possible values of $\ell, m$, the two angular symmetries, and both $\mathrm{HE}$ and $\mathrm{EH}$ modes. Equations (3.4)-(3.13) are valid in the limit $\delta \rightarrow 0$ except for the $\mathrm{TE}\left(\mathrm{e}_{Y}=0\right)$ mode expressions which are valid for arbitrary $\delta$. The $z$ components of the fields are of order $\sqrt{\delta}$ smaller than the transverse components, and can be neglected for the purposes of calculating power flow along the fibre. They are of importance in radiation problems and coupling caused by some irregularities (see Marcuse, 1974).

On transforming from a cylindrical to a rectangular coordinate system, the transverse fields are given by (Snyder, '1969a)

$$
\begin{aligned}
& \underline{e}_{p}=\left[ \pm \hat{x}\left\{\begin{array}{l}
\sin (\ell \overline{+} 1) \phi \\
\cos (\ell \overline{+} 1) \phi
\end{array}\right\}+\hat{\underline{y}}\left\{\begin{array}{c}
\cos (\ell \overline{+} 1) \phi \\
-\sin (\ell \overline{+}) \phi
\end{array}\right\} \quad \mathrm{f}_{\ell}(\mathrm{R})\right. \\
& =\left[\begin{array}{l}
\hat{x} \\
\hat{\underline{y}}
\end{array}\right] \quad f_{1}(R) \quad \mathrm{HE}_{1 \mathrm{~m}} \\
& =[-\underline{\hat{x}} \cos \phi-\hat{\underline{y}} \sin \phi] f_{0}(R) \quad T M M_{0 m} \\
& {[-\hat{x} \sin \phi+\hat{\underline{y}} \cos \phi] f_{0}^{\prime}(R), \quad T E_{0 m},}
\end{aligned}
$$


where $\underline{\hat{x}}, \hat{\underline{y}}$ are unit vectors. Because we have neglected the $z$ components, the fields are transversely linearly polarised, and the direction of polarisation depends, with the exception of the $\mathrm{HE}{ }_{1 \mathrm{~m}}$ modes, on the angular position $\phi$.

A full discussion of the modes of a step-index dielectric waveguide may be found in Marcuse (1974), Kapany and Burke (1972) and, for $\delta \ll 1$, in Snyder (1969, 1969a).

\subsection{DERIVATION AND VALIDITY OF THE DEGENERATE $\mathrm{P}_{\ell m}$ WAVEGUIDE MODES} The eigenvalue equation $(3.10)$ is valid in the limit $\delta \rightarrow 0$. From (3.10) and standard Bessel function recurrence relations, we find that the $\mathrm{HE}_{\ell m}$ and the $\mathrm{EH}_{\ell-2, \mathrm{~m}}$ modes are degenerate in this limit i.e. they have the same eigenvalues $U, W$ and therefore the same propagation constant $\beta$. Any linear combination of these two degenerate modes is then also a mode in that the transverse field pattern of the combination is preserved as it propagates along the waveguide. In general the $\mathrm{HE}_{\ell \mathrm{m}}$ and the $\mathrm{EH}_{\ell-2, \mathrm{~m}}$ modes are excited together (Snyder, 1969b).

We choose to take the sum and difference of the $\mathrm{HE}$ and $\mathrm{EH}$ modes; this results in modes with a position-independent, linear polarisation in either the $\underline{\hat{x}}$ or $\underline{\hat{y}}$ direction. These mode combinations have been observed on both optical fibres (Kapany; 1967; Midwinter, 1975) and visual photoreceptors (Enoch, 1963).

With the change in notation $\ell \rightarrow \ell+1$ so that $l$ now represents the azimuthal variation of the cartesian field components rather than the cylindrical components, the fields of the linear combinations of the $\mathrm{HE}$ and $\mathrm{EH}$ modes are given by $\mathrm{x}$-polarised, even and odd modes 


$$
\left(\psi_{\ell m}\right)^{\frac{1}{2}} \underline{e}^{x}(R, \phi)=\underline{\hat{x}} \gamma_{\ell} f_{\ell m}(R) \quad\left\{\begin{array}{l}
\cos \ell \phi \\
\sin \ell \phi
\end{array}\right\}
$$

and $\mathrm{y}$-polarised, even and odd modes

$$
\left(\psi_{\ell m}\right)^{\frac{i}{2}} \underline{e}^{y}(R, \phi)=\hat{\underline{y}} \gamma_{\ell}{ }^{f_{l m}}(R) \quad\left\{\begin{array}{l}
\cos \ell \phi \\
\sin \ell \phi
\end{array}\right\},
$$

where $\gamma_{\ell}=1$ for $\ell=0$ and $\sqrt{2}$ for $\ell \geqslant 1$. Gloge (1971) denotes these modes as $\mathrm{LP} \mathrm{lm}^{\prime}$, the LP standing for linearly-polarised. However this is to some extent misleading because the original $\mathrm{HE}, \mathrm{EH}$ modes are also linearly polarised; we use the notation $\mathrm{P}_{\ell m}$ for these modes.

The $\mathrm{m}$-dependence has been explicitly included in the expressions for $\mathrm{f}_{\ell \mathrm{m}}$ and the normalisation constant $\psi_{\ell \mathrm{m}}$. For the $\mathrm{P}_{\ell \mathrm{m}}$ modes

$$
\begin{aligned}
f_{\ell m}(R) & =\frac{J_{\ell}\left(U_{\ell m}{ }^{R}\right)}{J_{\ell}{ }^{\left(U_{\ell m}\right)}} \quad, R \leqslant 1 \\
& =\frac{K_{\ell}\left(W_{\ell m} R\right)}{K_{\ell}{ }^{\left(W_{\ell m}\right)}}, \quad R \geqslant 1 .
\end{aligned}
$$

where

$$
\mathrm{v}^{2}=\mathrm{U}_{l \mathrm{~m}}^{2}+\mathrm{W}_{\ell \mathrm{m}}^{2}
$$

and

$$
\frac{U_{\ell m} J_{\ell+1}\left(U_{\ell m}\right)}{J_{\ell}{ }^{\left(U_{\ell m}\right)}}=\frac{W_{\ell m} K_{\ell+1}\left(W_{\ell m}\right)}{K_{\ell}{ }^{\left(W_{\ell m}\right)}}
$$

is the eigenvalue equation. The normalisation constant, $\psi_{\ell m}$ ' defined so that

$$
\begin{aligned}
\int_{A_{\infty}} \underline{e}_{p} \times \underline{h}_{-q}^{*} \cdot \hat{z} d A & =\left(\frac{\varepsilon_{1}}{\mu}\right)^{\frac{3}{2}} \int_{A_{\infty}} e_{p} \cdot e_{q}^{*} d A \\
& =\delta p q
\end{aligned}
$$

is given by 


$$
\psi_{\ell m}=\pi \rho^{2}\left(\frac{\varepsilon_{1}}{\mu}\right)^{\frac{1}{2}}\left(\frac{\mathrm{V}}{\mathrm{U}_{\ell m}}\right)^{2} \frac{\mathrm{K}_{\ell-1}\left(\mathrm{~W}_{\ell \mathrm{m}}\right) \mathrm{K}_{\ell+1}\left(\mathrm{w}_{\ell \mathrm{m}}\right)}{\mathrm{K}_{\ell}^{2}\left(\mathrm{~W}_{\ell \mathrm{m}}\right)}
$$

Mode patterns for the first few ${ }{ }_{\ell m}$ modes are shown in Fig. 3.2. The propagation constant $\beta_{\ell m}$ for the $\mathrm{P}_{\ell m}$ modes is given by

$$
\begin{aligned}
\left(\rho \beta_{l m}\right)^{2} & =\left(\rho \mathrm{k}_{1}\right)^{2}-\mathrm{U}_{l \mathrm{~m}}^{2} \\
& =\frac{v^{2}}{\delta}\left[1-\delta\left(\frac{\mathrm{U}_{\ell \mathrm{m}}}{\mathrm{V}}\right)^{2}\right] \\
& \approx \frac{\mathrm{v}^{2}}{\delta}
\end{aligned}
$$

The transverse eigenvalue $U_{\ell m}$ is plotted as a function of $V$ in Fig. 3.3, from which can be found $\beta_{\ell m}$ using $(3.26)$ and $w_{\ell m}$ using $(3.22)$.

In summary, the $\mathrm{P}_{\ell m}$ modes:

(1) have a simple form which leads to an analytic expression for the coupling coefficient (2.23);

(2) have real field vectors given by (3.18), (3.19), with $\underline{h}_{\mathrm{p}}=\left(\frac{\varepsilon_{1}}{\mu}\right)^{\frac{1}{2}} \hat{\mathrm{z}} \times \mathrm{e}_{\mathrm{p}}$. The $\mathrm{z}$ components are much smaller than the transverse components and are neglected;

(3) are linearly polarised in either the $\hat{\underline{x}}$ or $\hat{\underline{y}}$ directions; the polarisation is independent of position;

(4) form an orthonormal set of bound modes;

(5) have only one l,m pair for each cutoff value, compared with two $\left(\mathrm{HE}_{\ell+1, \mathrm{~m}^{\prime}} \mathrm{EH}_{\ell-1, \mathrm{~m}}\right.$ ) for the usual waveguide modes;

(6) have four possible configurations $(\underline{\hat{x}}$ or $\underline{\hat{y}}$, sin $\ell \phi$ or $\cos \ell \phi)$ for each $\ell, m$ pair, except for $\ell=0$ which has two $(\underline{\hat{x}}$ or $\underline{\hat{y}}$ ).

(7) The $\mathrm{HE}_{1 \mathrm{~m}}$ modes are already degenerate and become the Pom modes. 


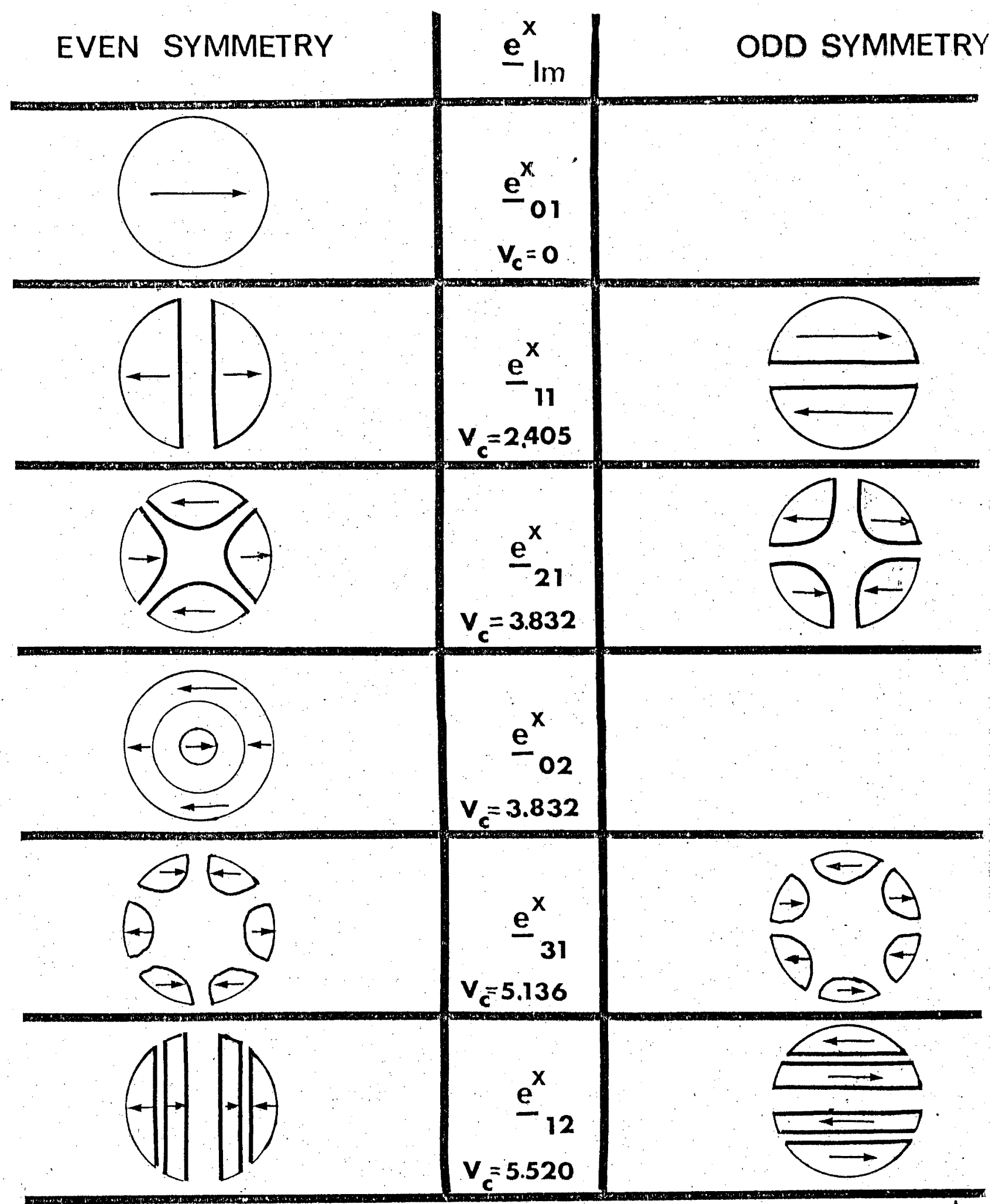

Fig. 3.2. Modal patterns for the first $\operatorname{six} \mathrm{P}_{\ell_{m}}$ modes.

$\mathrm{V}_{\mathrm{C}}$ is the cutoff value of $\mathrm{V}$. The $\mathrm{x}$ polarisation

is shown: to obtain the $\mathrm{y}$-polarisation rotate the

electric vectors through $90^{\circ}$. 


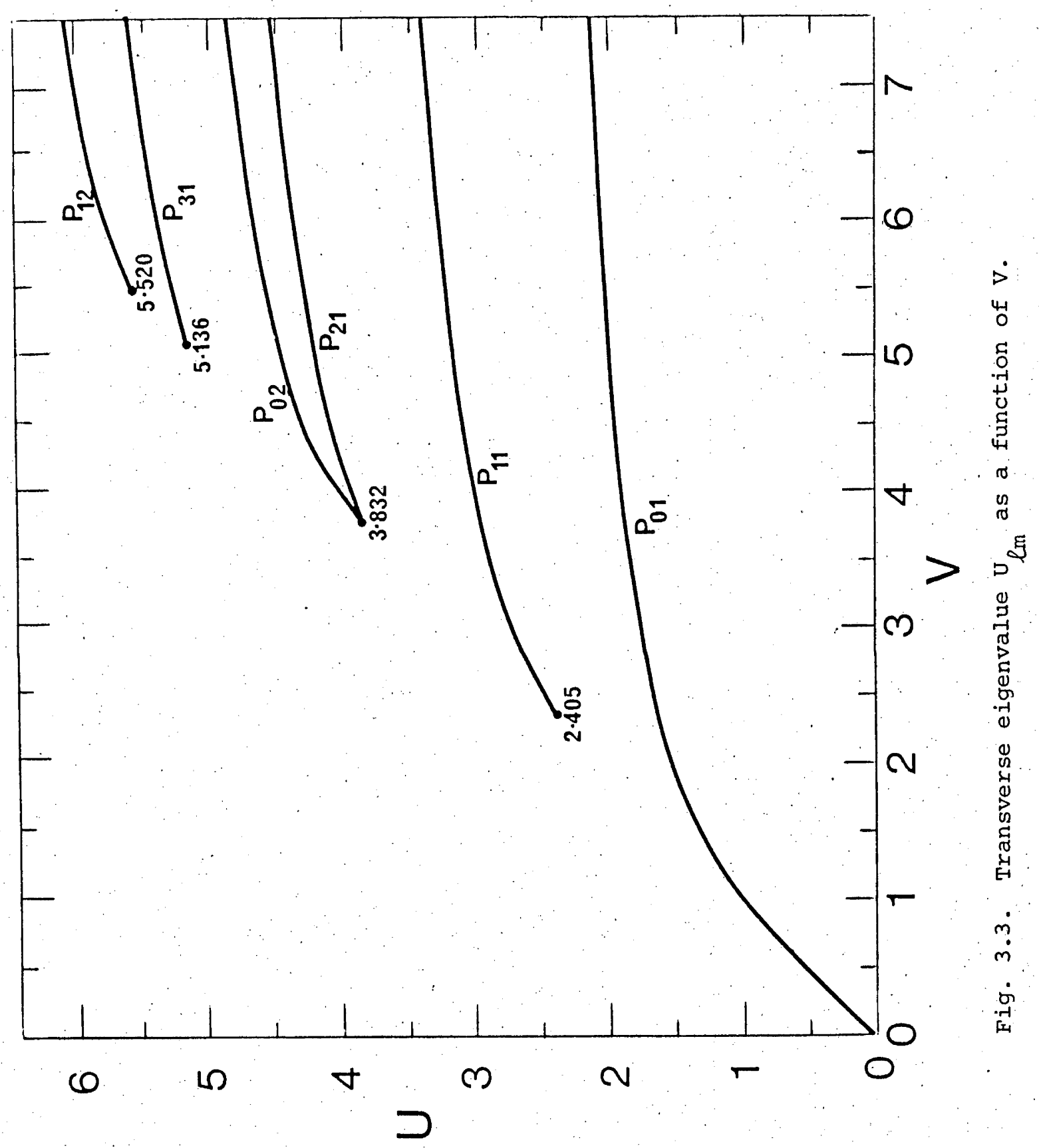


In the limit $\delta \rightarrow 0$, results for the horizontal and vertical polarisations are identical. For arbitrary $\delta$, this is not the case (see Jones, 1965; Marcuse, 1971).

In deriving the ${ }^{P_{l m}}$ modes, we assume that the propagation constants of the component $\mathrm{HE}_{\ell+1, \mathrm{~m}}$ and $\mathrm{EH}_{\ell-1, \mathrm{~m}}$ modes are identical. If $\delta \neq 0$ there is a small difference in the propagation constants, so that the $\mathrm{HE}$ and $\mathrm{EH}$ modes do not remain in phase throughout the length of a long waveguide; under these conditions the $\mathrm{P}_{\text {lm }}$ mode is no longer a true mode (Snyder and Pask, 1972; Wijngaard, 1971). We can of course always express the waveguide field in terms of $P$ modes even when $\delta \neq 0$, because (together with the continuous modes) they form a complete, orthogonal set of modes (Marcuse, 1973). However this introduces a complexity into the coupling problem which is unnecessary. for our purposes. Instead we find over what length of waveguide the $P$ modes are valid.

We have (for one angular symmetry),

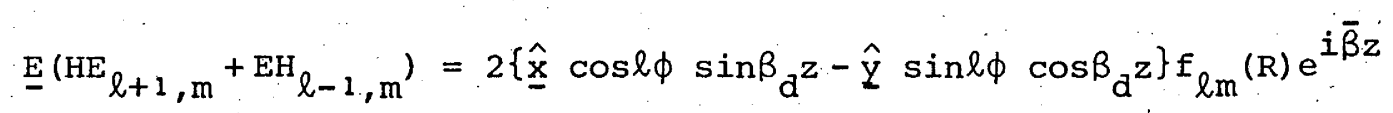

where

$$
\begin{aligned}
\bar{\beta} & =\frac{\beta_{\mathrm{HE}}+\beta_{\mathrm{EH}}}{2} \approx \beta_{\mathrm{HE}}, \\
\beta_{\mathrm{d}} & =\frac{\beta_{\mathrm{HE}}-\beta_{\mathrm{EH}}}{2}
\end{aligned}
$$

depend on $l$ and $m$. Similar expressions are found for the difference and for the other angular symmetry. Inspection of (3.28) shows that unless $\beta_{d}=0(\delta=0$ is a sufficient condition), there is a beating between the $\mathrm{x}$ - and $\mathrm{y}$ - polarised modes corresponding to the non-degeneracy of the 
$\mathrm{HE}$ and $\mathrm{EH}$ modes. However, for a waveguide of length $\ell$, the $\mathrm{HE}_{\ell+1, \mathrm{~m}}$ and $\mathrm{EH}_{\ell-1, \mathrm{~m}}$ modes are effectively degenerate if $\left|\beta_{d}\right| \ell<\frac{\pi}{2}$.

Therefore the $P$ modes remain valid for those waveguides for which

$$
\frac{\ell}{\rho} \ll \bar{L}=\frac{\pi}{2 \rho\left|\beta_{\mathrm{d}}\right|}
$$

$\overline{\mathrm{I}}$ as a function of $\mathrm{V}$ for several values of $\delta$, is plotted in Fig. 3.4 for the modes $P_{11}, P_{21}$. $\beta_{d}$ is found by solving the full eigenvalue equation $(\delta \neq 0)$ (Collin, 1961) numerically for $\mathrm{U}_{\mathrm{HE}}(\delta), \mathrm{U}_{\mathrm{EH}}(\delta)$, and using the relationship (3.26) between $U$ and $\beta$.

The mode $\mathrm{P}_{11}$ is formed from combinations of $\mathrm{HE}_{21}$ and $\mathrm{TE}_{01}$ or $\mathrm{TM}_{01}$ modes. These two combinations show different behaviour because the TM eigenvalues are functions of $\delta$, whereas the TE eigenvalues are not. The $\mathrm{P}_{11}$ curves in Fig. 3.4 are the $\mathrm{HE} \pm \mathrm{TM}$ combinations which have $\overline{\mathrm{L}} \rightarrow \infty$ at $\mathrm{V} \approx 3.6$, where the curves for $\mathrm{U}_{\mathrm{HE}}(\delta), \mathrm{U}_{\mathrm{TM}}(\delta)$ cross (Snyder, 1969). The HE $\pm T E$ combinations have $\bar{L}$ curves similar to those for the $P_{21}$ modes. A useful upper bound for $\beta_{d}$ can be found by observing that for the first few modes, $\left|\mathrm{U}_{\mathrm{HE}}^{2}-\mathrm{U}_{\mathrm{EH}}^{2}\right| \lesssim\left|\mathrm{U}_{\mathrm{TM}}^{2}-\mathrm{U}_{\mathrm{TE}}^{2}\right| \cdot$ Using the asymptotic expansions for $\mathrm{U}_{\mathrm{TM}^{\prime}} \mathrm{U}_{\mathrm{TE}}$ in Snyder (1971a), we find

$$
\mathrm{U}_{\mathrm{TM}}^{2}-\mathrm{U}_{\mathrm{TE}}^{2} \approx \frac{2 \delta}{\mathrm{V}} \mathrm{U}^{2}
$$

Then

$$
\begin{aligned}
2 \rho\left|\beta_{\mathrm{d}}\right| & \approx \frac{\sqrt{\delta}}{2 \mathrm{~V}}\left|\mathrm{U}_{\mathrm{HE}}^{2}-\mathrm{U}_{\mathrm{EH}}^{2}\right| \\
& <\delta^{3 / 2}\left(\frac{\mathrm{U}}{\mathrm{V}}\right)^{2} \\
& <\delta^{3 / 2}
\end{aligned}
$$

where $U=U_{T E}=U(\delta=0)$. Equation (3.34) provides an upper bound for most modes but (3.33) is not valid for $\mathrm{P}_{31}$. Some exact curves for $\overline{\mathrm{L}}$ as a function of $\delta$, together with the approximations, are plotted in Fig. 3.5. 


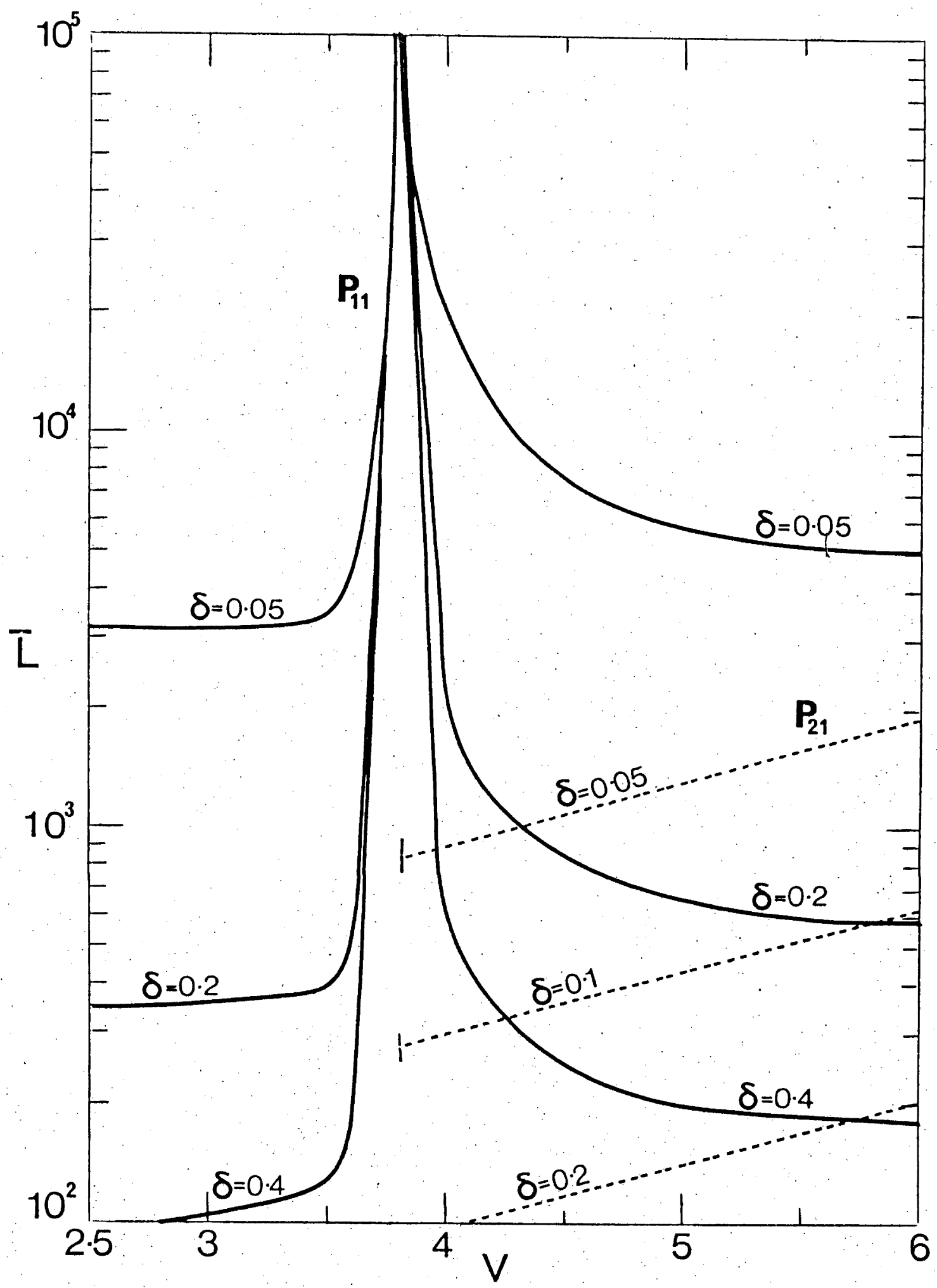

Fig. 3.4. Upper bound $\bar{L}$ on the length of fibre for which the $P_{11}$ (solid line) and $P_{21}$ (dashed line) modes are valid, as a function of $\mathrm{V}$ and the dielectric difference $\delta$. The modes are valid along a fibre of length $l$ and radius $\rho$ if $\ell / \rho \ll \bar{L}$. 


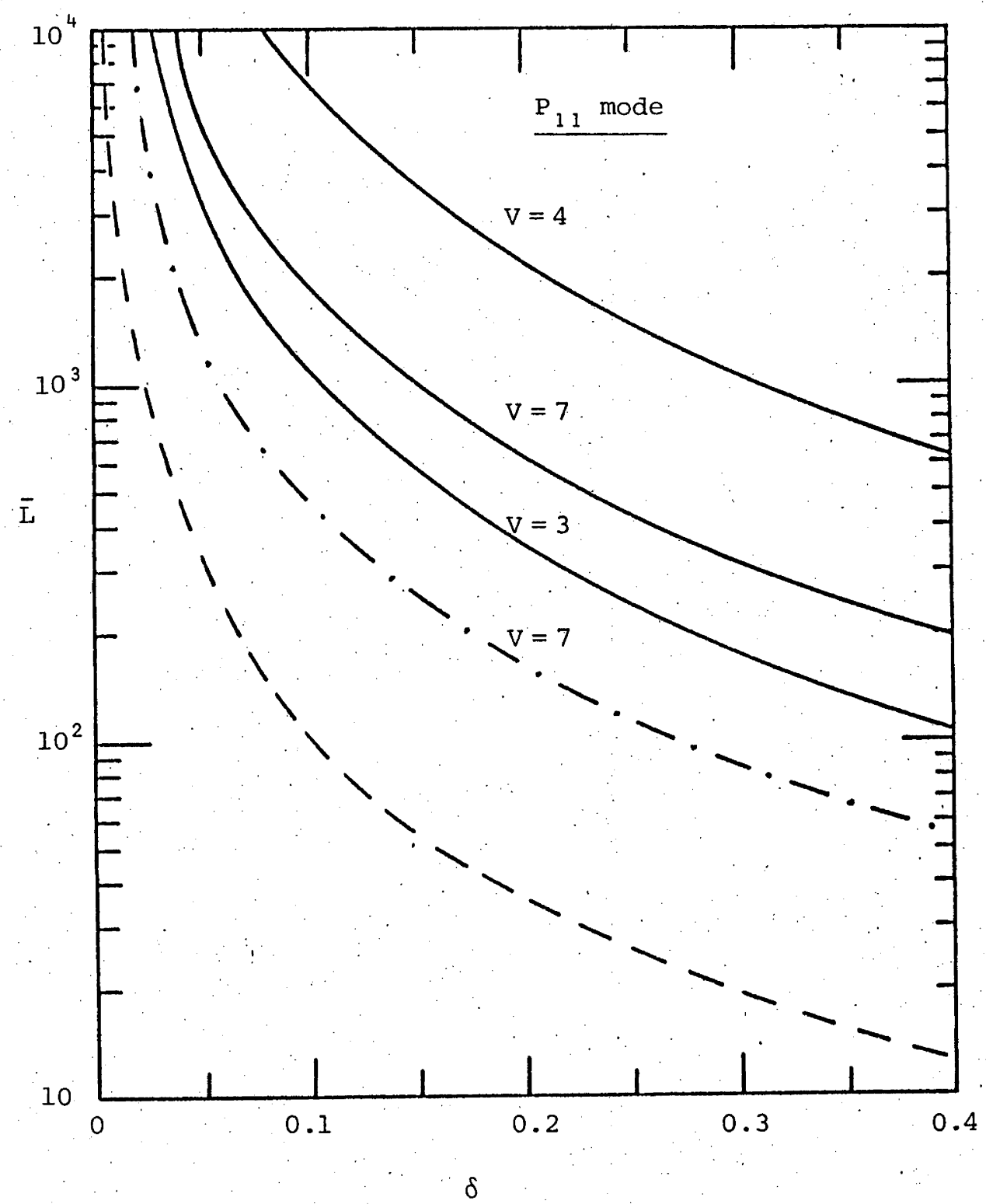

Fig. 3.5. Normalised length of validity $\bar{L}$ as a function of the dielectric difference $\delta$, for a $\mathrm{P}_{11}$ $\left(\mathrm{HE}_{21} \pm \mathrm{TM}_{01}\right)$ mode. Also shown are the approximations $\pi / \delta^{3 / 2}(\div--\longrightarrow$ and $\left(\frac{V}{U}\right)^{2} \frac{\pi}{\delta^{3 / 2}}$ with $\mathrm{V}=7$ (- - - $\rightarrow$.

The curves with $\mathrm{V}=3,4$ for this approximation lie below the $V=7$ curve. 


\subsection{POWER TRANSFER BETWEEN TWO OPTICAL FIBRES}

The coupled-mode theory developed in Chapter 2 can now be applied to coupling or crosstalk between neighbouring dielectric waveguides or optical fibres, using the simplified circular-waveguide modes derived in Section 3.4. We consider first two fibres assumed to be lossless, parallel and uniform in the z-direction. The geometry of the two-fibre system is given in Fig. 3.6.

\subsubsection{Coupling Coefficient}

The coupling coefficient between mode 1 on fibre 1 and mode 2 on fibre 2 is given by $(2.23)$ as

$$
c_{12}=-\frac{\omega}{2}\left(n_{2}^{2}-n^{2}\right) \int_{A_{2}} e_{1} \cdot e_{2} d A,
$$

where the (real) $\mathrm{P}_{\ell \mathrm{m}}$ mode fields (3.18), (3.19) are to be used. It can be seen from the form of the fields that, with the scalar dielectric constant used here, the $\underline{x}$ and $\underline{y}$ modes do not couple, and when $\alpha=0$, the sine and cosine modes also do not couple.

We allow for arbitrary radii and refractive indexes (subject to the restraint $n_{1}, n_{2} \approx_{n} ; n_{1}, n_{2}>_{n}$ ) so that the parameters $\mathrm{v}_{1}, \mathrm{v}_{2}$ are related by

$$
\frac{v_{1}}{v_{2}}=\frac{n_{1} \rho_{1}}{n_{2} \rho_{2}}\left(\frac{\delta}{\delta_{2}}\right)^{\frac{1}{2}}
$$

Substitution of the fields (3.18), using the cosine modes, into (3.35) leads to

$$
\begin{aligned}
C_{12}=- & \frac{\omega}{2} \frac{\left(n_{2}^{2}-n^{2}\right) \gamma_{1} \gamma_{2} \rho_{2}^{2}}{\sqrt{\psi_{1} \psi_{2}} K_{\ell_{1}}\left(W_{1}\right) J_{\ell_{2}}\left(U_{2}\right)} \int_{0}^{1} R_{2} d R_{2} J_{\ell_{2}}\left(U_{2} R_{2}\right) k_{\ell_{1}}\left(w_{1} R_{1}\right) \\
& \times \int_{0}^{2 \pi} d \phi_{2} \cos \ell_{1} \phi_{1} \cos \ell_{2} \phi_{2}
\end{aligned}
$$




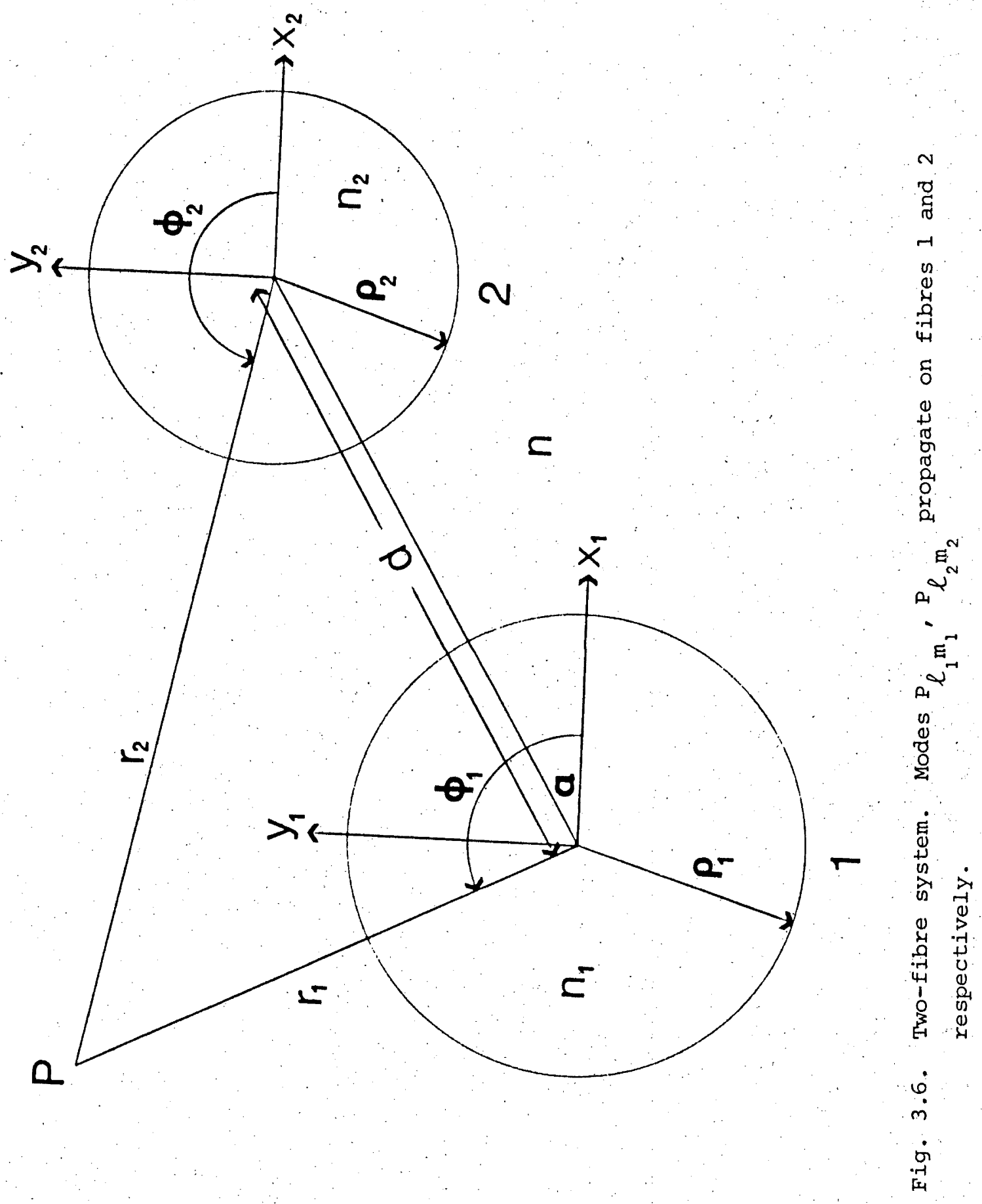


where mode 1 is characterised by $\ell_{1}, m_{1}, U_{1}, W_{1}$, mode 2 by $\ell_{2}, m_{2}, U_{2}, w_{2}$ and $R_{i}=\frac{r_{i}}{\rho_{i}}$. We use the Bessel function addition formula

$$
K_{\ell_{1}}\left(W_{1} R_{1}\right) e^{i \ell_{1} \phi_{1}^{\prime}}=\sum_{j=-\infty}^{\infty} K_{\ell_{i}+j}\left(W_{1} D_{1}\right) I_{j}\left(\frac{W_{1} \rho_{2} R_{2}}{\rho_{1}}\right) e^{i j \phi_{2}^{\prime}},
$$

which relates the coordinates $r_{1}, \phi_{1}^{\prime}=\phi_{1}+\alpha$ to $r_{2}, \phi_{2}^{\prime}=\pi-\left(\phi_{2}+\alpha\right)$, to write the evanescent field of fibre 1 in terms of the coordinates of fibre 2. Here $D_{1}=d / \rho_{1}$. Under this translation

$$
\begin{aligned}
\cos \ell_{1} \phi_{1} & \rightarrow \cos \left(j\left[\pi-\left(\phi_{2}+\alpha\right)\right]-\ell_{1} \alpha\right) \\
& =(-1)^{j} \cos \left[j \phi_{2}+\left(\ell_{1}+j\right) \alpha\right],
\end{aligned}
$$

which gives the angle integration in (3.37) as

$$
\begin{aligned}
& (-1)^{j} \int_{0}^{2 \pi} \cos \ell_{2} \phi_{2} \cos \left[j \phi_{2}+\left(\ell_{1}+j\right) \alpha\right] d \phi_{2} \\
& \quad=(-1)^{\ell_{2}} \pi\left[\delta_{j, l_{2}} \cos \left(l_{1}+l_{2}\right) \alpha+\delta_{j,-l_{2}} \cos \left(l_{1}-l_{2}\right) \alpha\right]
\end{aligned}
$$

where $\delta_{i, j}$ is the Kronecker delta. Putting (3.39) back into (3.37) and using $I_{-\ell}=I_{\ell}$, we have

$$
\begin{aligned}
& C_{12}=-\frac{\pi \omega}{2} \frac{n_{2}^{2}-n^{2}}{\sqrt{\psi_{1} \psi_{2}}} \frac{\gamma_{1} \gamma_{2} \rho_{2}^{2}}{K_{\ell_{1}}\left(w_{1}\right) J_{\ell_{2}}\left(U_{2}\right)}(-1)^{\ell_{2}} \\
& \times\left[K_{\ell_{1}+l_{2}}\left(w_{1} D_{1}\right) \cos \left(l_{1}+l_{2}\right) \alpha+K_{\ell_{1}-l_{2}}\left(w_{1} D_{1}\right) \cos \left(\ell_{1}-\ell_{2}\right) \alpha\right] I,
\end{aligned}
$$

where

$$
\begin{gathered}
I=\int_{0}^{I} R_{2} d R_{2} J_{\ell_{2}}\left(U_{2} R_{2}\right) I_{\ell_{2}}\left(\frac{W_{1} \rho_{2} R_{2}}{\rho_{1}}\right) \\
=\left(\frac{1}{U_{2}^{2}+\bar{W}_{2}^{2}}\right) \frac{J_{l_{2}}\left(U_{2}\right)}{K_{\ell_{2}}\left(W_{2}\right)}\left\{\bar{W}_{2} I_{\ell_{2}+1}\left(\bar{W}_{2}\right) K_{\ell_{2}}\left(W_{2}\right)+W_{2} I_{\ell_{2}}\left(\bar{W}_{2}\right) K_{\ell_{2}+1}\left(W_{2}\right)\right\}
\end{gathered}
$$

Here 


$$
\overline{\mathrm{W}}_{2}=\frac{\mathrm{W}_{1} \rho_{2}}{\rho_{1}} \text {, }
$$

and we have used the eigenvalue equation (3.23) and a standard Bessel function integration formula.

$$
\kappa_{12}=-\frac{\left(\rho_{1} \rho_{2}\right)^{\frac{1}{2}} C_{12}}{\left(\delta_{1} \delta_{2}\right)^{\frac{1}{4}}}
$$

Using $(3.25),(3.40),(3.42)$ we find

$$
\begin{aligned}
& K_{12}=(-1)^{\ell_{2}}\left(\frac{\gamma_{1} \gamma_{2}}{2}\right) \frac{U_{1} U_{2}}{V_{1}}\left(\frac{V_{2}}{V_{1}}\right)^{\frac{1}{2}} \\
& \times \frac{\left[K_{\ell_{1}+l_{2}}\left(W_{1} D_{1}\right) \cos \left(l_{1}+l_{2}\right) \alpha+K_{\ell_{1}-\ell_{2}}\left(W_{1} D_{1}\right) \cos \left(\ell_{1}-\ell_{2}\right) \alpha\right]}{\left[K_{\ell_{1}-1}\left(W_{1}\right) K_{\ell_{1}+1}\left(W_{1}\right) K_{\ell_{2}-1}\left(W_{2}\right) K_{\ell_{2}+1}\left(W_{2}\right)\right]^{\frac{1}{2}}} \\
& \times \frac{\left[\bar{W}_{2} I_{\ell_{2}+1}\left(\bar{W}_{2}\right) K_{\ell_{2}}\left(W_{2}\right)+W_{2} I_{\ell_{2}}\left(\bar{W}_{2}\right) K_{\ell_{2}+1}\left(W_{2}\right)\right]}{\left[U_{2}^{2}+\bar{W}_{2}^{2}\right]}
\end{aligned}
$$

To obtain $k_{21}$, exchange the subscripts 1 and 2 except in the $(-1)^{l_{2}}$. This form of the coupling coefficient is given for the sake of generality, as it can be used whenever the $\mathrm{P}_{\ell m}$ modes are valid. Further simplification will be given in Section 3.5 .3 after we have used (3.45) to investigate the validity of the coupled-mode formalism of Chapter 2 as applied to two circular dielectric waveguides.

\subsubsection{Validity of the Formulation for Two Circular Waveguides}

In Section 2.3, we introduced the expression

$$
\mathrm{P}_{\mathrm{D}} \approx\left(\mathrm{T}+2 \mathrm{XI_{12 }}\right) \mathrm{F} \sin ^{2} B_{\mathrm{b}} \mathrm{z}
$$

as the term necessary to ensure conservation of power by the coupled mode equations. 


$$
\begin{aligned}
T & =\frac{C_{12}-C_{21}}{C_{12}} \\
& =\frac{K_{12}-K_{21}}{K_{12}},
\end{aligned}
$$

is a measure of the power in the coupling mechanism, and

$$
2 X I_{12} \approx \frac{4 \Delta \beta}{\delta \beta}
$$

is a measure of the power in the cross-terms, which arise because of the non-orthogonality of the modes. We required that $P_{D} \ll 1$ for the power in the system to be given by

$$
\mathrm{P}=\frac{1}{2}\left\{\left|\mathrm{a}_{1}\right|^{2}+\left|\mathrm{a}_{2}\right|^{2}\right\}
$$

and that $T+2 X I_{12} \ll I$ for $P_{D} \ll P_{2}$, the power in the unexcited fibre. Using (3.45), we have calculated $T$ and $F$ as a function of the normalised difference between the propagation constants $\Delta \beta^{\prime}=\frac{\rho_{1} \Delta \beta}{\sqrt{\delta_{1}}}$. We present curves for $T$ and $F$ in Figs: 3.7-3.10. We can rewrite (3.49) as

$$
2 \times I_{12} \approx \frac{4 \Delta \beta^{\prime}}{V_{1}}
$$

Changes in $\Delta \beta^{\prime}$ are generated in two ways: (a) by varying the ratio $\rho_{1} / \rho_{2}$ and (b) by varying $\delta_{2}$, given $\delta_{1}$. We find here that the functions are, to a good approximation, independent of $\delta_{1}$ and therefore depend only on $\delta_{2} / \delta_{1}$. Curves are plotted to cover only the range of parameters for which the error is significant and the coupling not negligible i.e. small $\mathrm{V}_{1}$ and/or small separation. For larger values of these parameters, the power transfer $F$ falls off very rapidly with $\Delta \beta^{\prime}$, and consequently the error is negligible over the range in which coupling is significant. In Fig. 3.11, we plot $T+2 X I_{12}$ and $P_{D}$ as a function of $\Delta \beta^{\prime}$ 


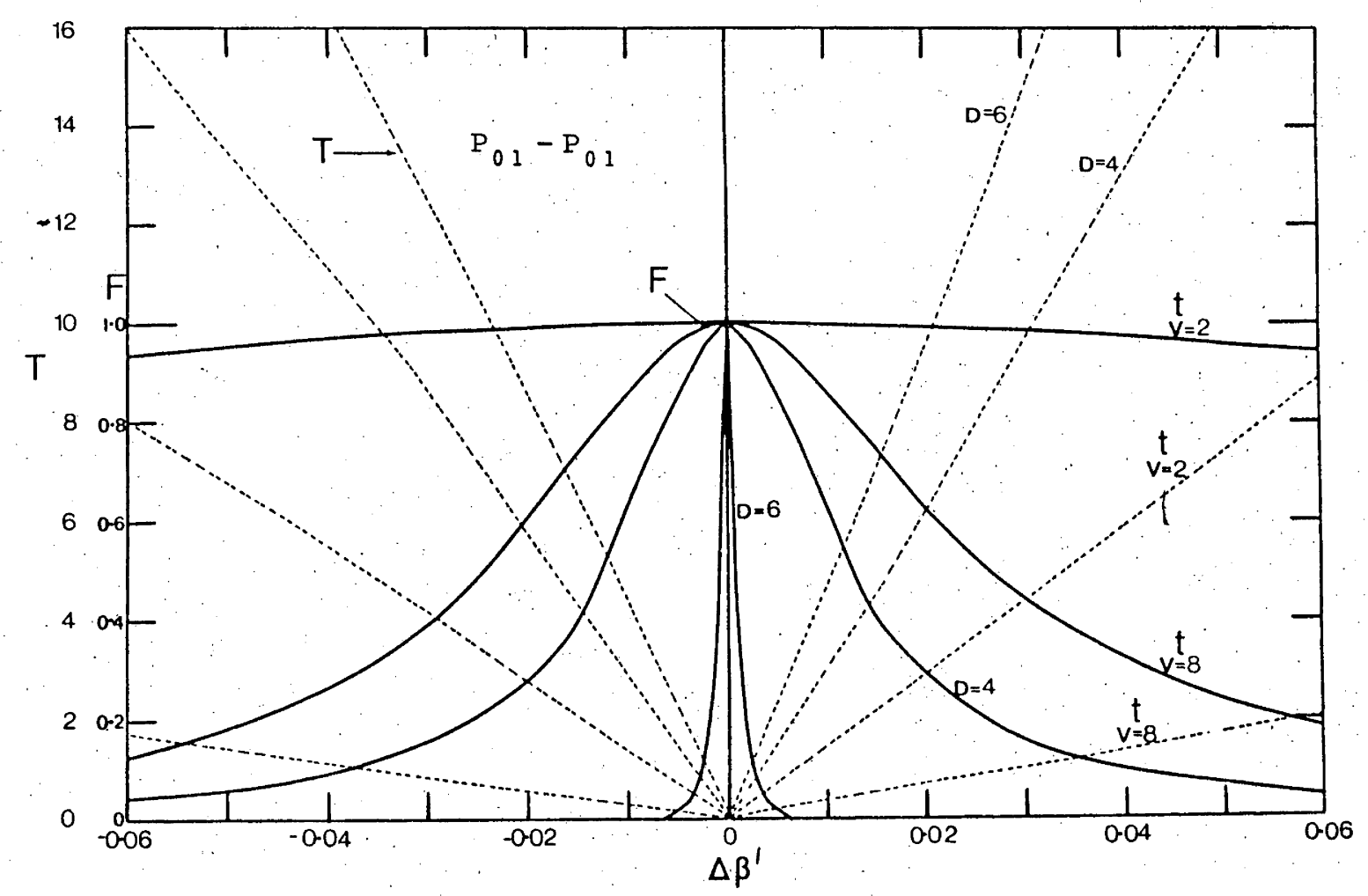

Fig. 3.7. The maximum power transfer $\mathrm{F}$ (solid line) and the error $\mathrm{T}$ in percent (dashed line) as a function of $\Delta \beta^{\prime}=\rho_{1}\left(\beta_{1}-\beta_{2}\right) / \sqrt{\delta_{1}}$ for coupling between two $\mathrm{P}_{01}$ modes. Unless stated otherwise $\mathrm{V}\left(=\mathrm{V}_{1}\right)=2$. For coupling between like modes, the two ways of varying $\Delta \beta^{\prime}$ (see text) give almost identical curves. The changes in $\rho_{1} / \rho_{2}$ or $\delta_{1} / \delta_{2}$ corresponding to the changes in $\Delta \beta^{\prime}$ and expressed as a percentage of their values when $\Delta \beta^{\prime}=0$, are $\Delta\left(\rho_{1} / \rho_{2}\right)=80 \Delta \beta^{\prime} \%, \Delta\left(\delta_{2} / \delta_{1}\right)=-67 \Delta \beta^{\prime} \%$. 


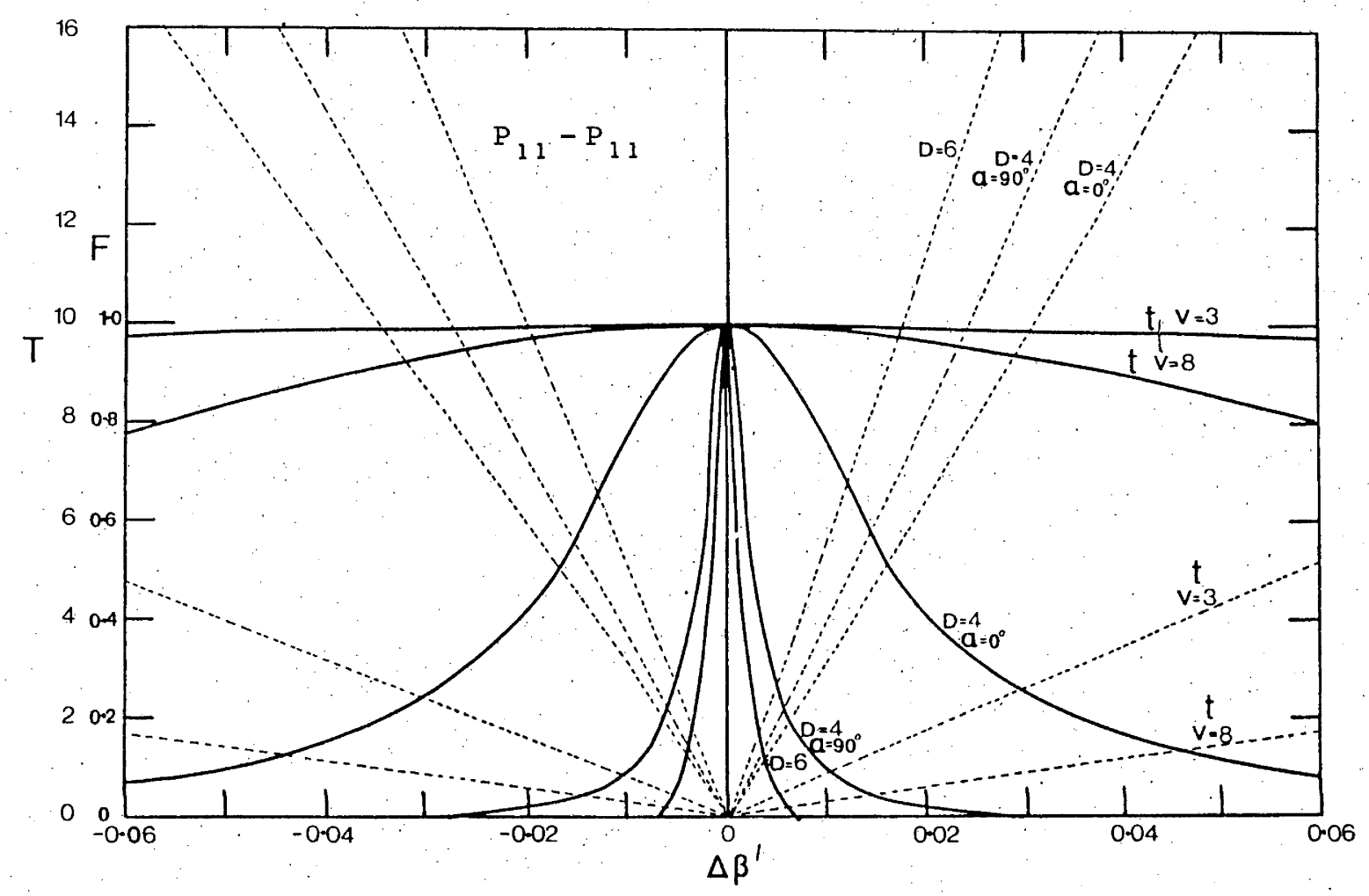

Fig. 3.8. As for Fig. 3.7, but for coupling between $\mathrm{P}_{11}$ modes. $\mathrm{v}_{1}=3$ and $\alpha=0^{\circ}$ unless otherwise indicated. Here $\Delta\left(\rho_{1} / \rho_{2}\right)=34 \Delta \beta ! \%, \Delta\left(\delta_{2} / \delta_{1}\right)=-50 \Delta \beta^{\prime}$ \% . 


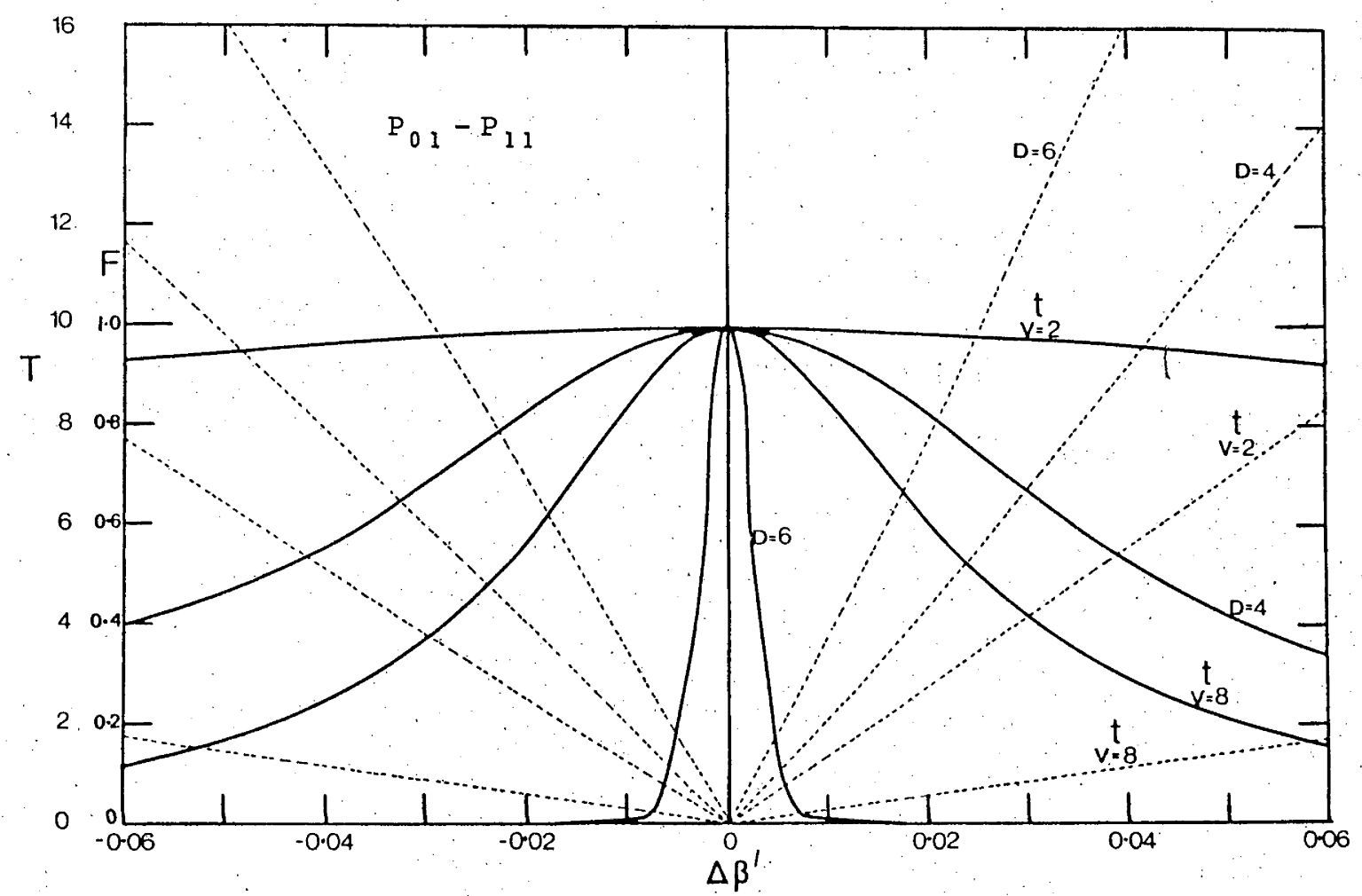

Fig. 3.9. As for Fig. 3.7, but for coupling between a $P_{01}$ mode (fibre 1) and $a P_{11}$ mode (fibre 2).. For coupling between unilike modes the two ways of varying $\Delta \beta^{\prime}$ give different curves. Here $n_{1}=n_{2}\left(\delta_{1}=\delta_{2}\right)$ and the ratio $\rho_{1} / \rho_{2}$ is varied. $\Delta\left(\rho_{1} / \rho_{2}\right)=60 \Delta \beta^{\prime} \%$. 


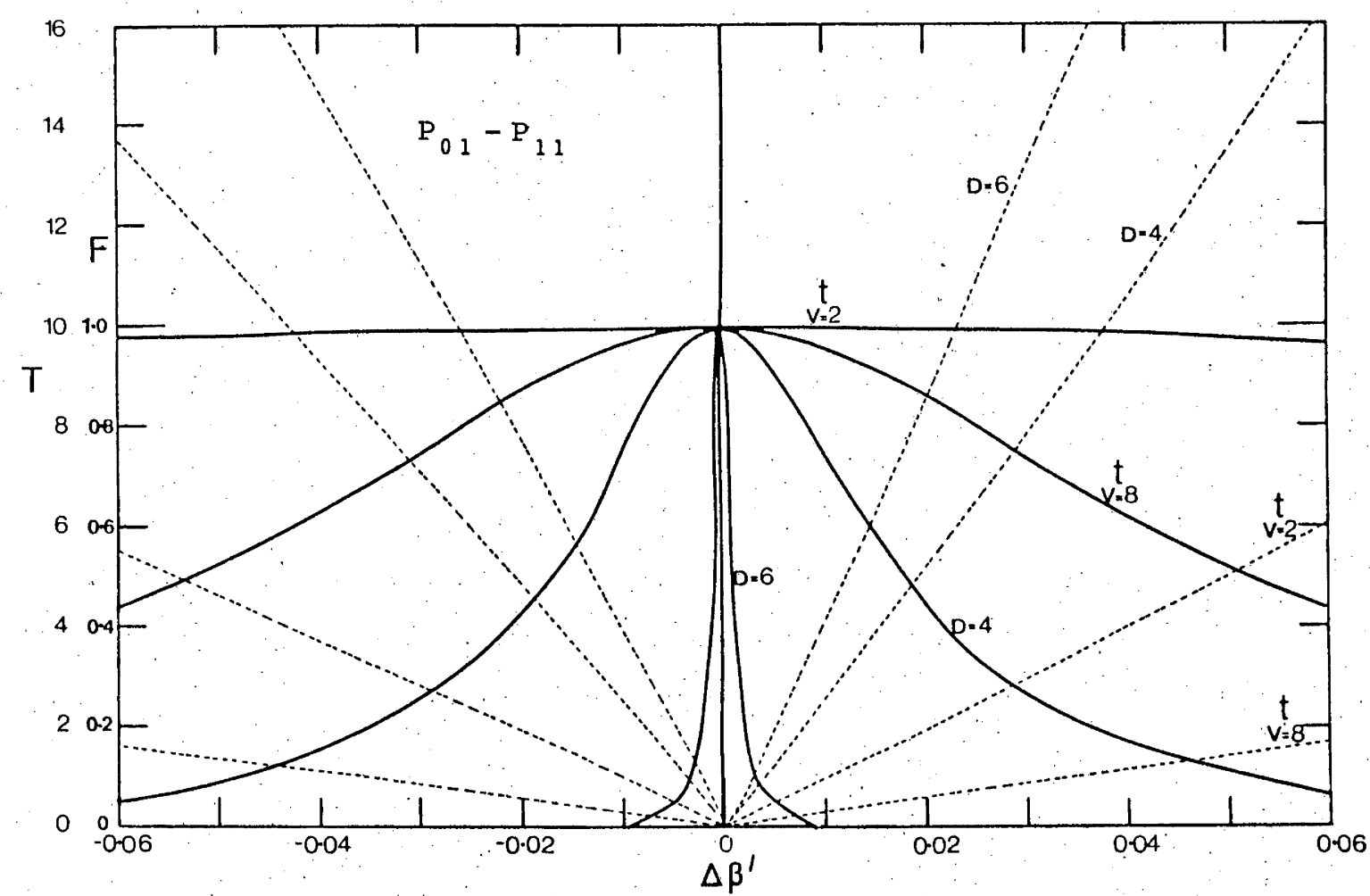

Fig. 3.10. As for Fig. 3.9, but $\rho_{1}=\rho_{2}$ and $\delta_{2}$ is varied. To a good approximation the curves are independent of the value of $\delta_{1}$ used. $\Delta\left(\delta_{2} / \delta_{1}\right)=-34 \Delta \beta ! \%$. 


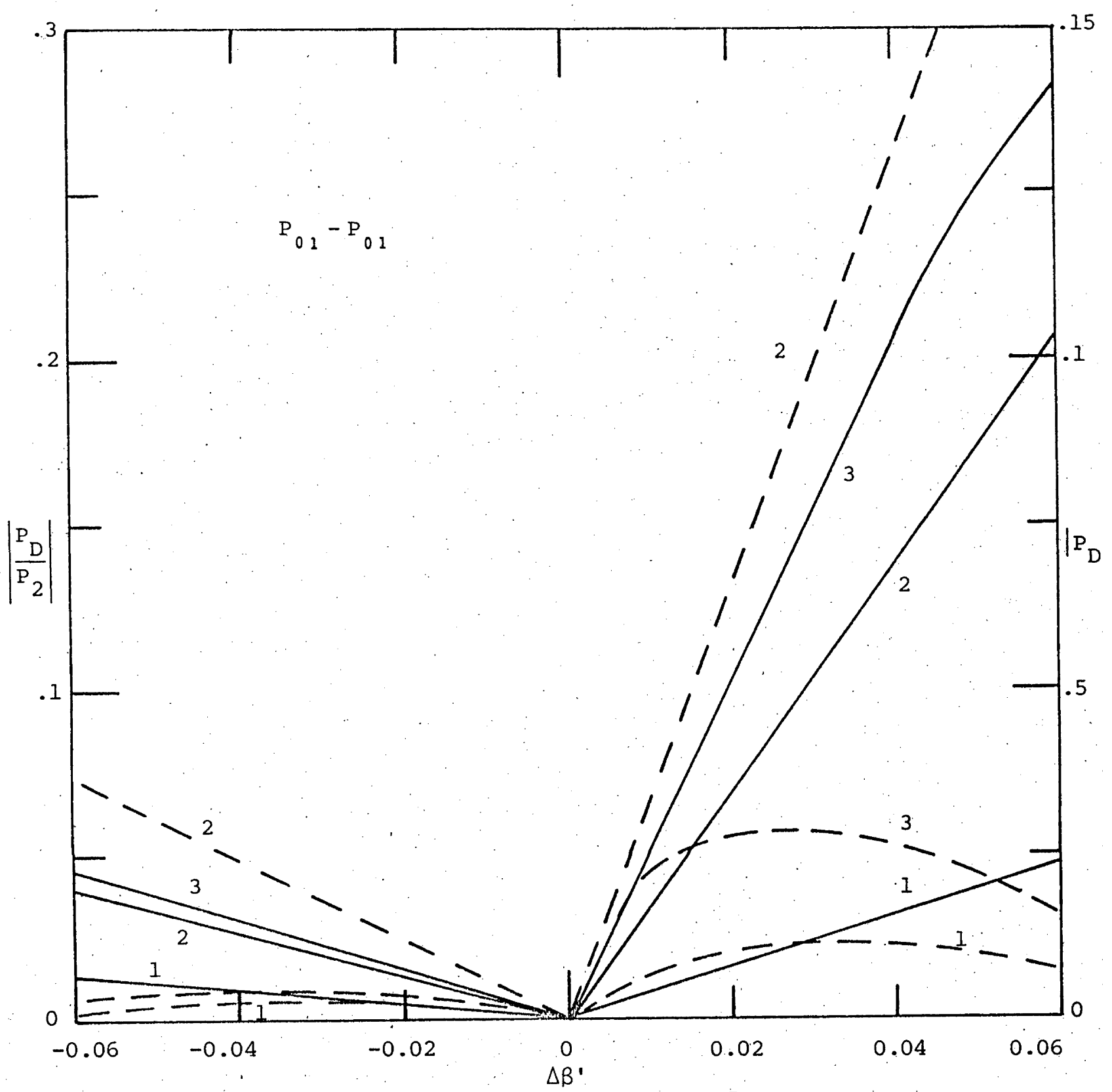
Fig. 3.11. $\left|\mathrm{P}_{\mathrm{D}}\right|(--$ right-hand scale $)$ and $\left|\frac{\mathrm{P}_{\mathrm{D}}}{\mathrm{P}_{2}}\right|(-$ $\Delta \beta^{\prime}=\frac{\rho_{1} \Delta \beta}{\sqrt{\delta_{1}}}$ for $P_{01}$ modes. We have taken $\sin ^{2} \beta_{b} z=1$.

The curves are

1. $\quad v_{1}=8$ and touching fibres

$2 \quad v_{1}=2$ and touching fibres

3. $V_{1}=2$ and $D_{1}=d / \rho_{1}=4$.

The initial power in fibre 1 is 1 . 
for the case shown in Fig. 3.7. This shows over which regions the ratio $\mathrm{P}_{\mathrm{D}} / \mathrm{P}_{2}$ is significant, and explicitly when the term $\mathrm{P}_{\mathrm{D}} \ll 1$ (we take $\sin ^{2} \beta_{b} z=1$ to give maximum $P_{D}$ ).

We conclude that the two-equation form $(2.30),(2.31)$ of the coupled-mode equations is accurate whenever coupling between the modes is significant, except when $\beta_{1} \neq \beta_{2}$ and either of the modes is near cutoff with the fibres close together. The equations may still be used in these cases and the error determined from Figs. 3.7-3.11 (see also Section 3.5 .3 ).

A necessary assumption in our formulation is the weakcoupling assumption $|C| \ll|B|$. Breakdown of this condition means that the full normal-mode or some other approach must be used. To see that this is a reasonable assumption, we write

$$
\frac{\left|C_{i j}\right|}{\beta}=\frac{\sqrt{\delta}}{\rho \beta}\left|\kappa_{i j}\right| \leqslant \frac{\delta\left|\kappa_{i j}\right|}{v} .
$$

We find that $|\kappa| \lesssim 0.1$, so that if $\delta \sim 0.1,\left|C_{i j}\right| / \beta<\frac{0.01}{V}$. Therefore, for most practical purposes, the weak-coupling assumption is valid.

\subsubsection{Simplified Theory of Power Transfer}

The expression (3.45) for the coupling constant. $K$, although in a closed form, is cumbersome'. When $\beta_{1}=\beta_{2},(3.45)$ simplifies. Because

(1) coupling is significant oniy in a narrow range of $\Delta \beta$ about $\Delta \beta=0$, (Section 3.5.2),

(2) the coupling constant does not change greatly with $\Delta \beta$ in most cases (Figs. $3.7-3.10$ ), and 
(3) ratios of $\mathrm{C}^{\prime}$ 's and the geometric mean $\sqrt{\mathrm{C}_{12} \mathrm{C}_{21}}$ appear in $\beta_{b}$ and $F$, the simplified form may be used whenever coupling is significant.

Rewriting (3.26), we have

$$
\begin{aligned}
\beta^{2} & =\frac{V^{2}}{\rho^{2} \delta}-\frac{U^{2}}{\rho^{2}} \\
& =\frac{V^{2}}{\rho^{2} \delta}(1-\delta)+\frac{W^{2}}{\rho^{2}}
\end{aligned}
$$

where we have used (3.22). Then $\beta_{1}=\beta_{2}$ implies

$$
\begin{aligned}
\frac{w_{1}^{2}}{\rho_{1}^{2}}-\frac{w_{2}^{2}}{\rho_{2}^{2}} & =w^{2} \mu\left[n_{2}^{2}\left(1-\delta_{2}\right)-n_{1}^{2}\left(1-\delta_{1}\right)\right] \\
& =0
\end{aligned}
$$

from (3.12) and the definition of $\delta$. Therefore $W_{1} D_{1}=W_{2} D_{2}$ and $\bar{w}_{2}=w_{2}$ in (3.45) when $\beta_{1}=\beta_{2}$, and $\kappa=\kappa_{12}=\kappa_{21}$ is given by

$$
\begin{gathered}
K=(-1)^{l_{2}}\left(\frac{\gamma_{1} \gamma_{2}}{2}\right) \frac{\mathrm{U}_{1} \mathrm{U}_{2}}{\left(\mathrm{~V}_{1} \mathrm{~V}_{2}\right)^{3 / 2}} \\
\times \frac{\left[\mathrm{K}_{\ell_{1}}+\ell_{2}\left(\mathrm{~W}_{1} \mathrm{D}_{1}\right) \cos \left(\ell_{1}+l_{2}\right) \alpha+\mathrm{K}_{\ell_{1}-\ell_{2}}\left(\mathrm{~W}_{1} \mathrm{D}_{1}\right) \cos \left(\ell_{1}-\ell_{2}\right) \alpha\right]}{\left[\mathrm{K}_{\ell_{1}-1}\left(\mathrm{~W}_{1}\right) \mathrm{K}_{\ell_{1}+1}\left(\mathrm{~W}_{1}\right) \mathrm{K}_{\ell_{2}-1}\left(\mathrm{~W}_{2}\right) \mathrm{K}_{\ell_{2}+1}\left(\mathrm{~W}_{2}\right)\right]^{\frac{1}{2}}}
\end{gathered}
$$

where we have used the Wronskian identity

$$
I_{\ell+1}(W) K_{\ell}(W)+I_{\ell}(W) K_{\ell+1}(W)=\frac{1}{W}
$$

If the two fibres are identical, significant coupling takes place only between like modes; the difference in the propagation constants of the dielectric waveguide modes is in general too great for large power transfer between unlike modes. For coupling between like modes 
on identical fibres, the coupling constant reduces to

$$
K=(-1)^{\ell}\left(\frac{\gamma_{l}^{2}}{2}\right) \frac{U^{2}}{V^{3}} \frac{\left[K_{2 \ell}(W D) \cos 2 \ell \alpha+K_{0}(W D)\right]}{K_{\ell-1}(W) K_{\ell+1}(W)}
$$

We give curves of $\kappa$, calculated from (3.58), as a function of normalised separation $D$ and for different $V$ values for modes $P_{01}, P_{11}, P_{21}, P_{02}$ in Figs. $3.12-3.16$.

If the fibres are not identical, there is the possibility of significant coupling between unlike modes. By varying the radii or the refractive indexes of the fibres it is possible to adjust the propagation constants of the modes so that they are approximately equal. Similarly, on fibres of different refractive index for example, it is possible to adjust the radii so that like modes still have identical propagation constants (i.e. the same v). The values of $v_{2}$ as a function of $v_{1}$ and $\rho_{1} / \rho_{2}$ for equal propagation constants for like- and unlike-mode coupling between $P_{01}$ and $P_{11}$ modes are shown in Figs. 3.17-3.19. The curves are calculated from (3.5.5) and (3.23); the corresponding refractive index ratios can be found from (3.36).

In Figs. 3.20-3.23, we give curves for the coupling constant for coupling between $P_{01}, P_{11}$ modes on fibres of different radii.

In calculating the coupling constant we have used the even (cosine) modes, so that even-even coupling (Fig. 3.24) corresponds to $\alpha=0^{\circ}$, and for $P_{11}$ modes, for example

$$
k=-\frac{U^{2}}{v^{3}}\left[\frac{K_{2}(W D)+K_{0}(W D)}{K_{0}(W) K_{2}(W)}\right]
$$

We can include coupling between the odd (sine) modes by changing the angle $\alpha$; odd-odd coupling (Fig. 3.25) corresponds to $\alpha=90^{\circ}$, and $\kappa$ for the $\mathrm{P}_{11}$ modes in this case is given by 


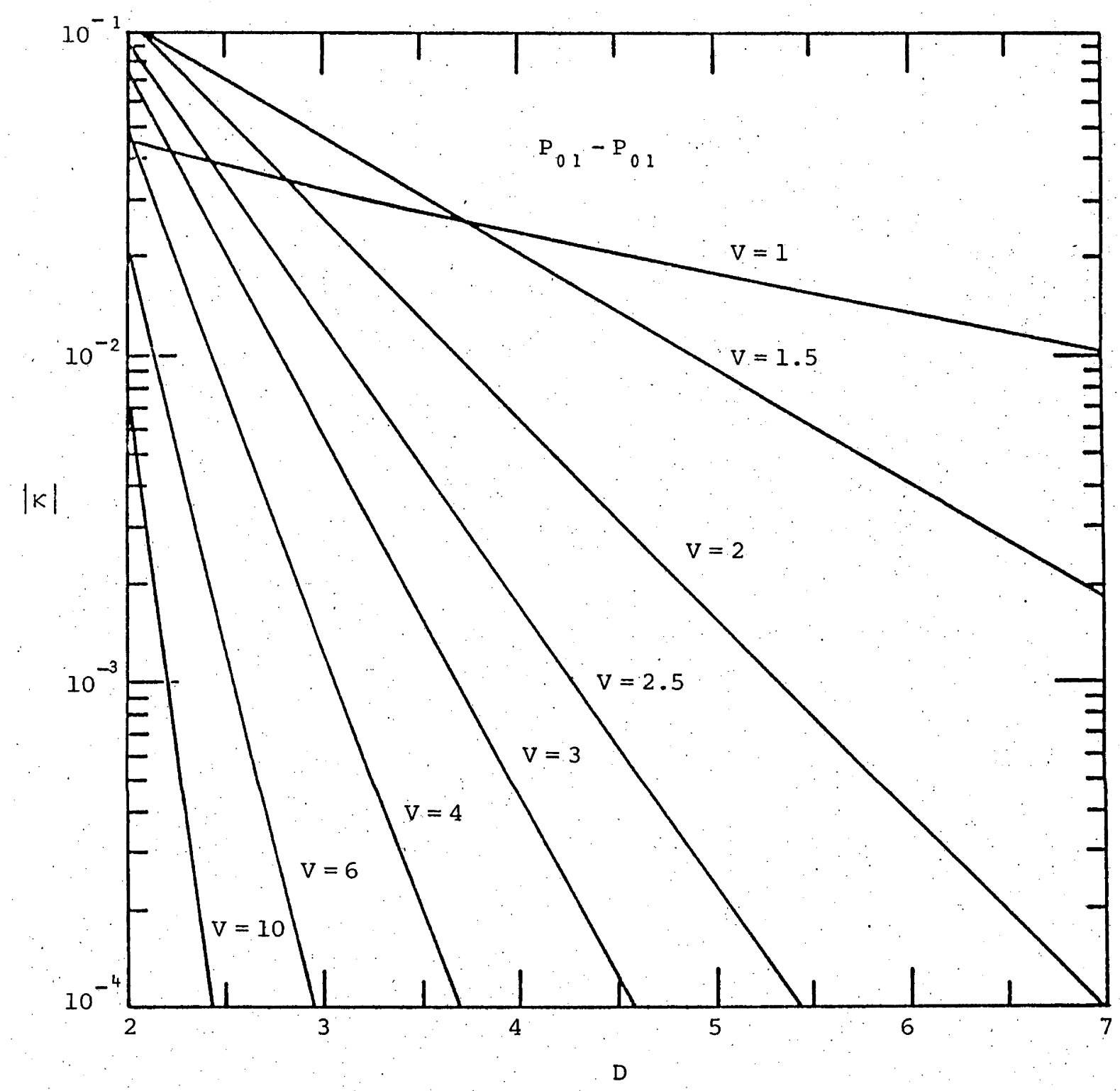

Fig. 3.12. The coupling constant $K$ as a function of normalised separation $D=d / \rho$, for $P_{01}$ modes on identical fibres. 


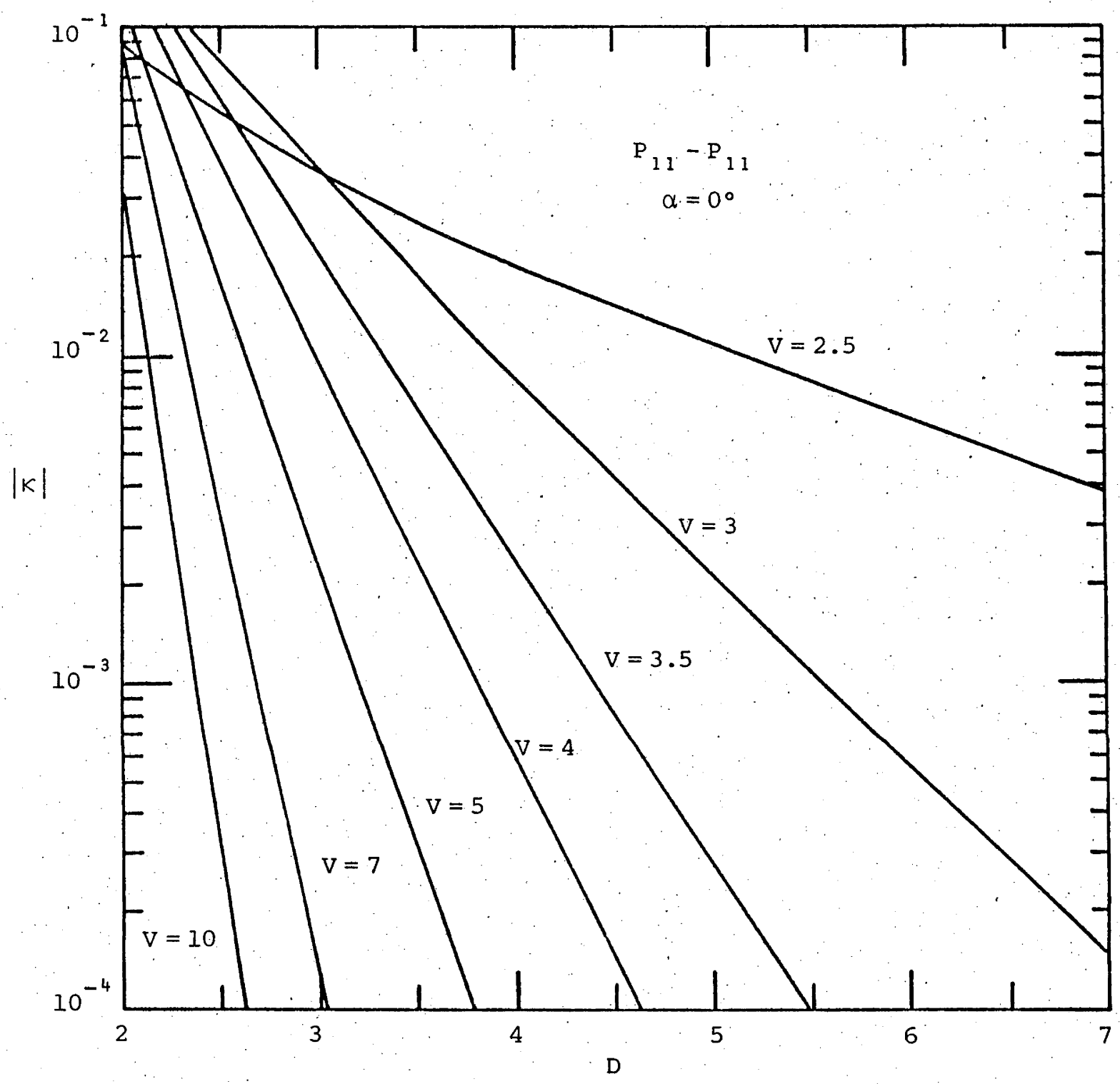

Fig. 3.13. The coupling constant $\kappa$ as a function of normalised separation $D=d / \rho$, for $P_{11}$ modes on identical fibres. $\alpha=0^{\circ}$ (even-even coupling). 


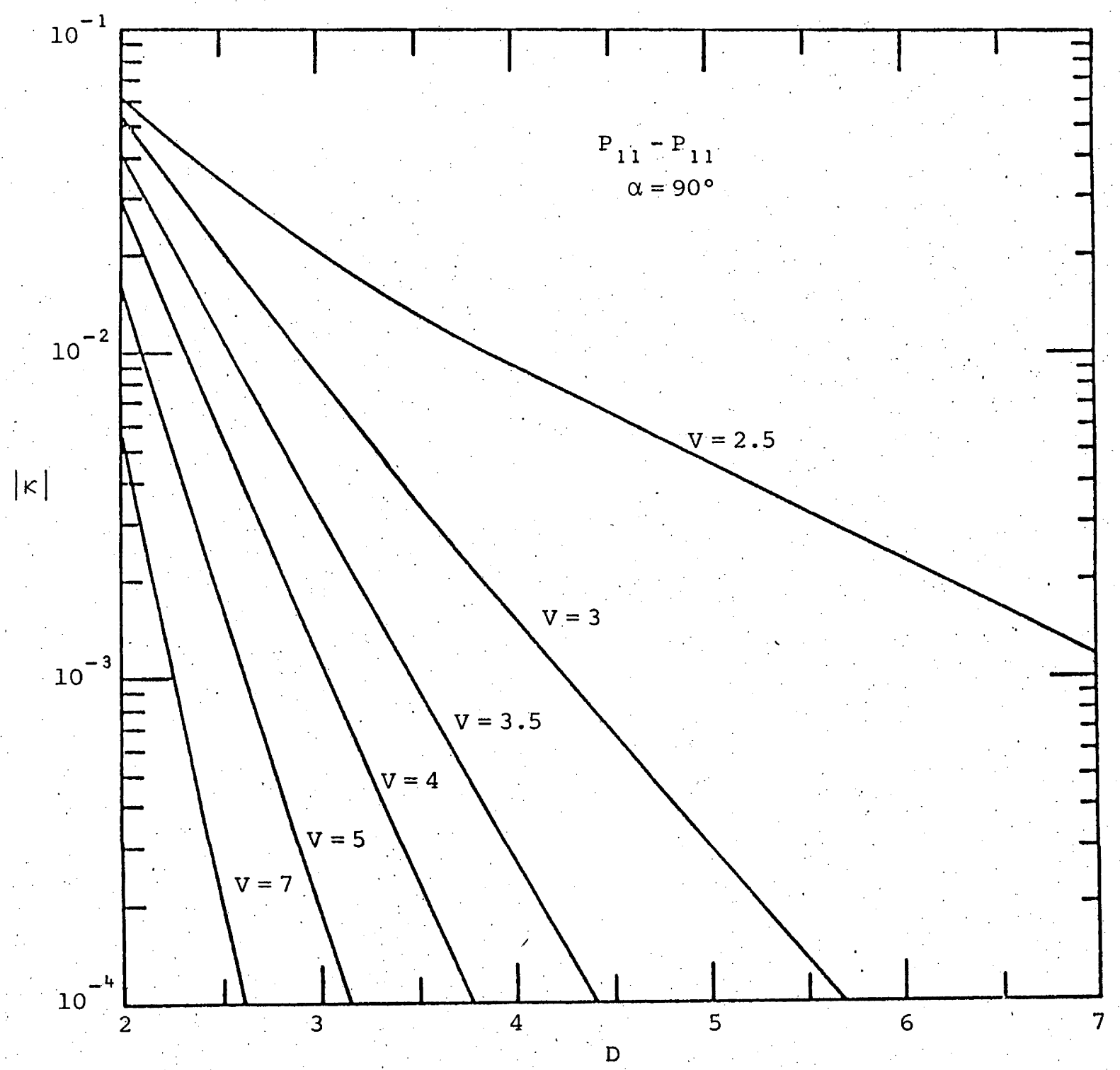

Fig. 3.14. The coupling constant $K$ as a function of normalised separation $D=d / \rho$, for $P_{11}$ modes on identical fibres. $\alpha=90^{\circ}$ (odd-odd coupling). 


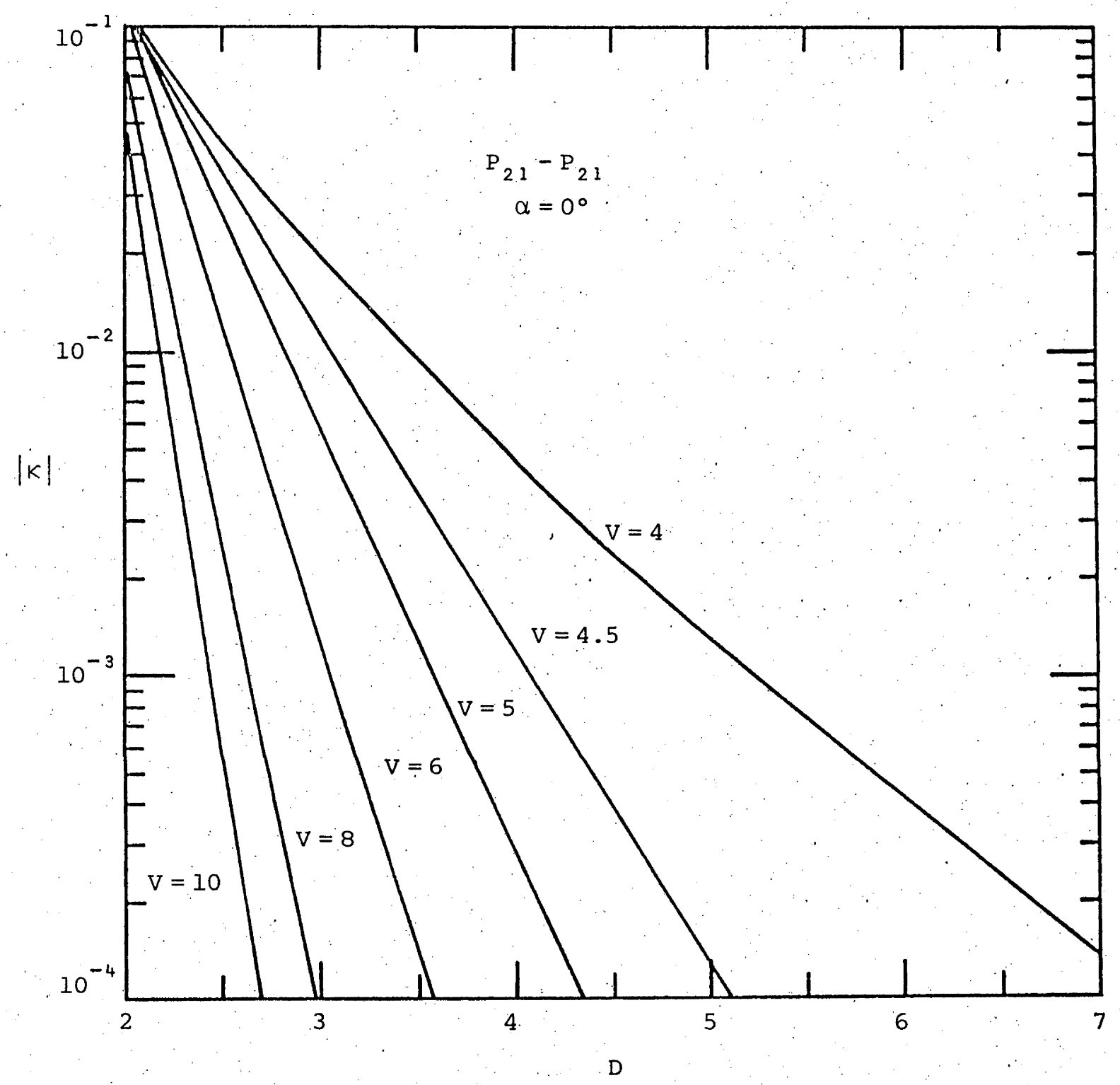

Fig. 3.15. The coupling constant $k$ as a function of normalised separation $D=d / \rho$, for $P_{21}$ modes on identical fibres. $\alpha=0^{\circ}$. 


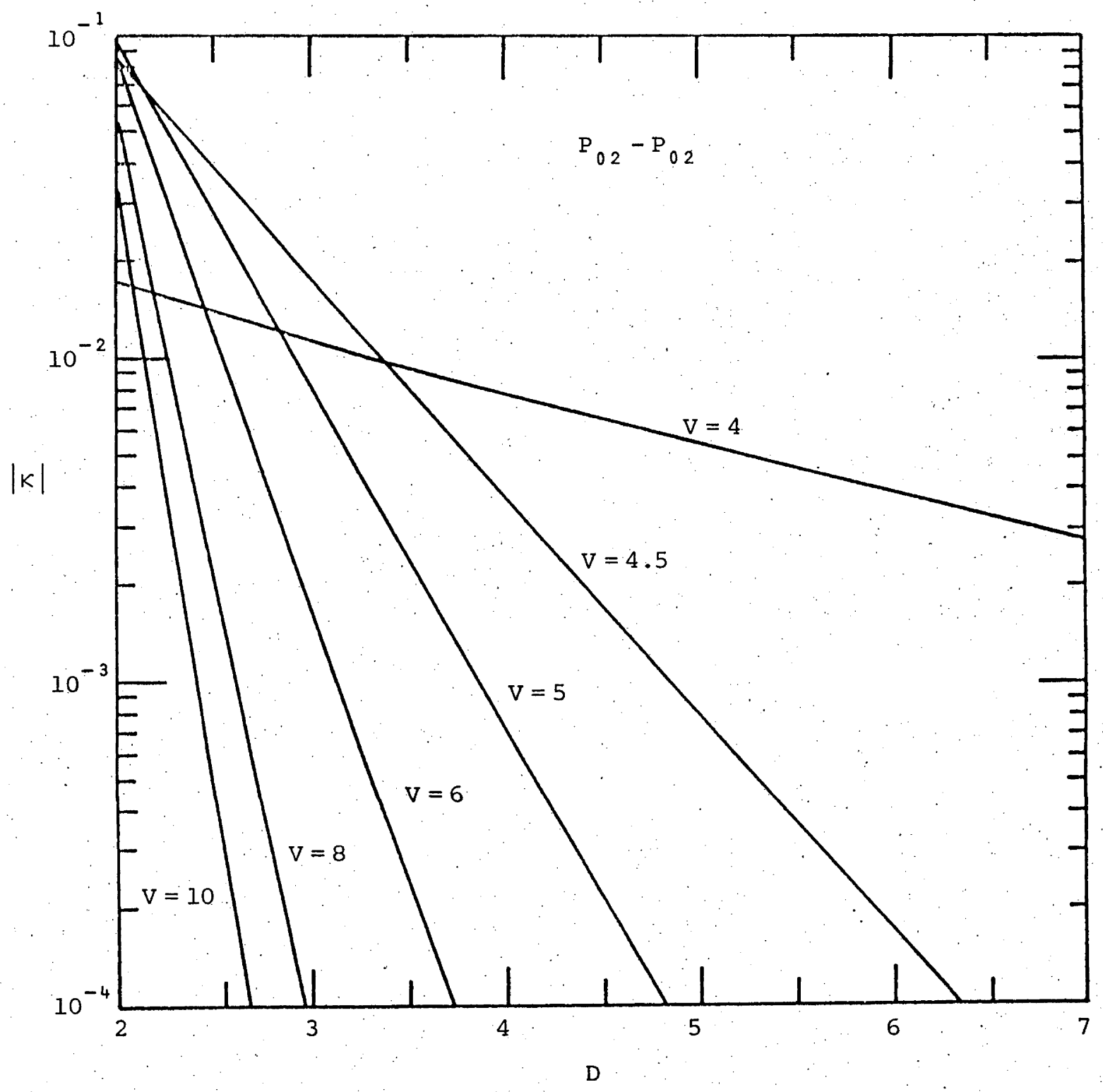

Fig. 3.16. The coupling constant $k$ as a function of normalised separation $D=d / \rho$, for $P_{02}$ modes on identical fibres. 


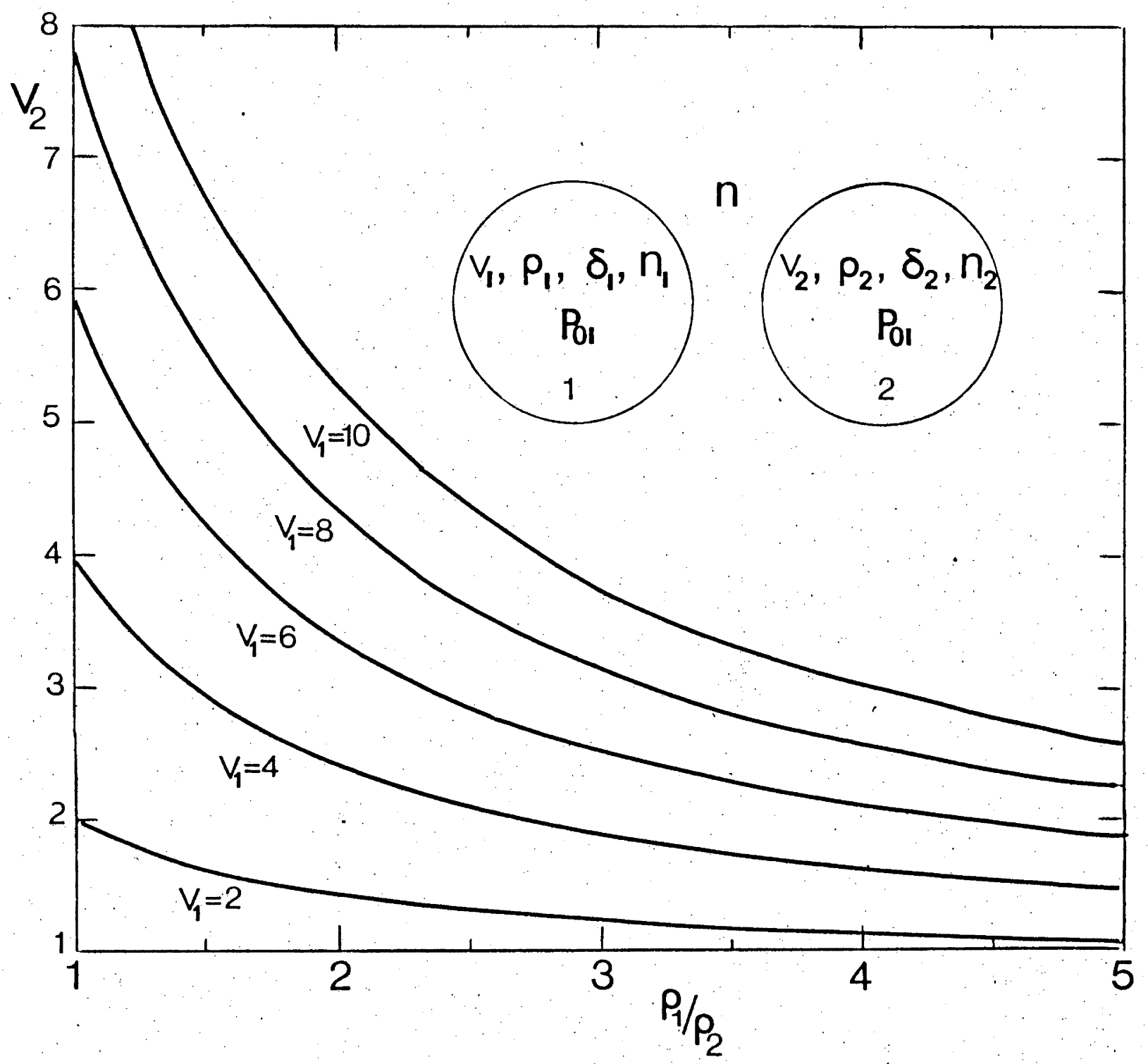

Fig. 3.17. Values of $v_{2}$ as a function of $v_{1}$ and $\rho_{1} / \rho_{2}$, that satisfy $\beta_{1}=\beta_{2}$ for the case of a: $P_{01}$ mode on each fibre. $v_{1}$ and $v_{2}$ are related by $v_{2} / v_{1}=\frac{\rho_{2}}{\rho_{1}}\left(\frac{\varepsilon_{2} \delta_{2}}{\varepsilon_{1} \delta_{1}}\right)^{\frac{1}{2}}$. 


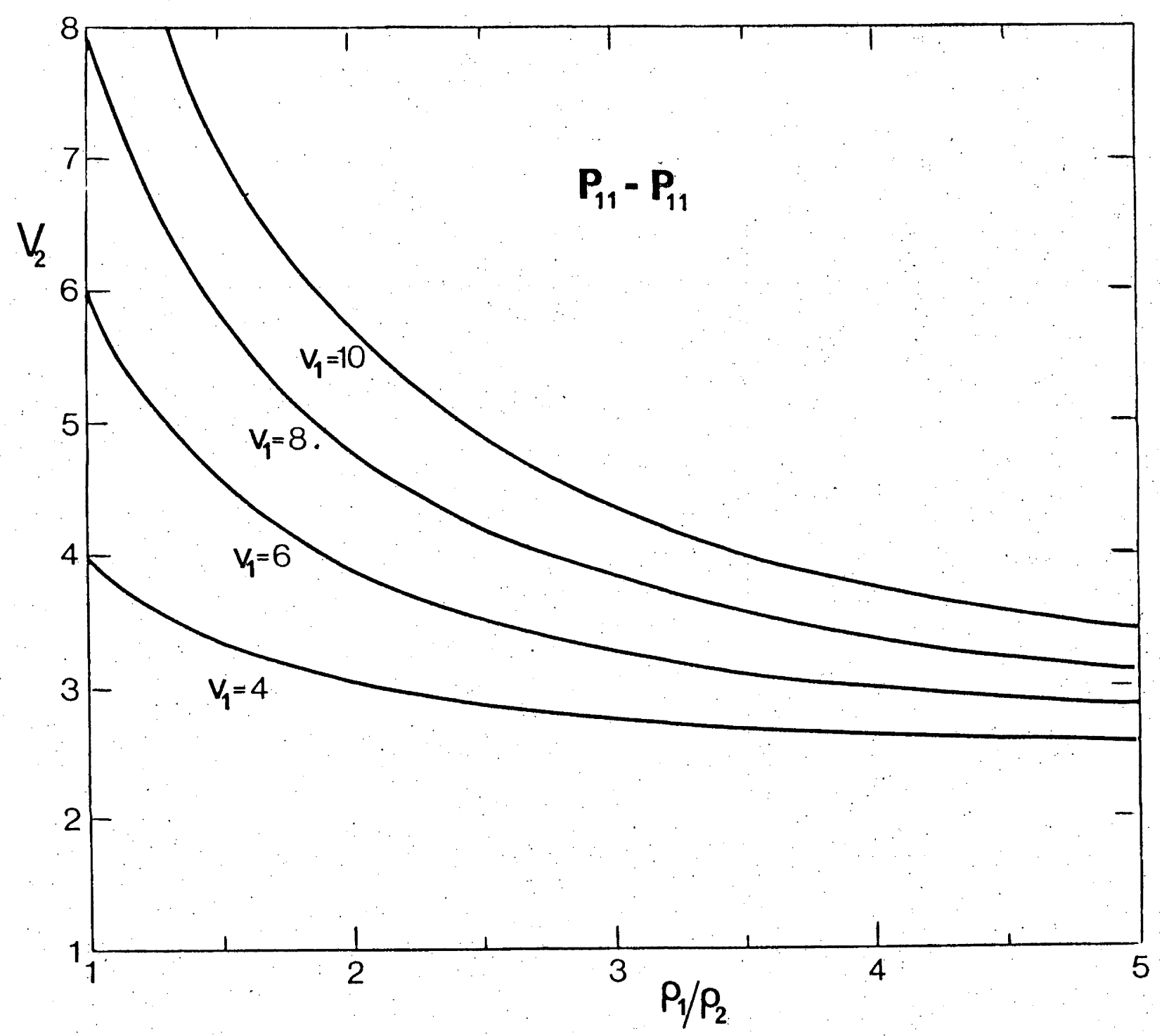

Fig. 3.18. As for Fig. 3.17, but with a $P_{11}$ mode on each fibre. 


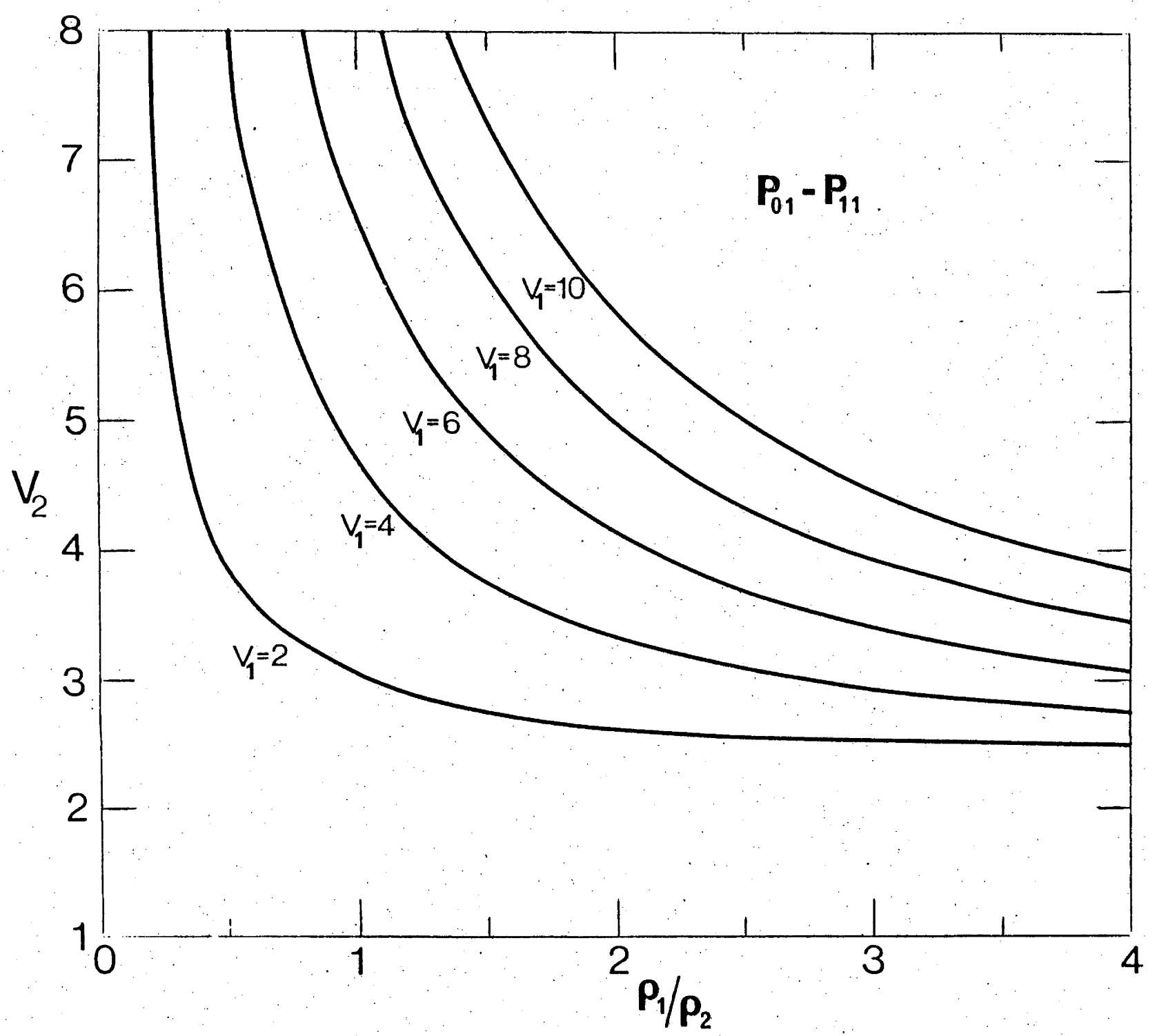

Fig. 3.19. As for Fig. 3.17, but with a $P_{0.1}$ mode on fibre 1 and $a P_{11}$ mode on fibre 2 . 


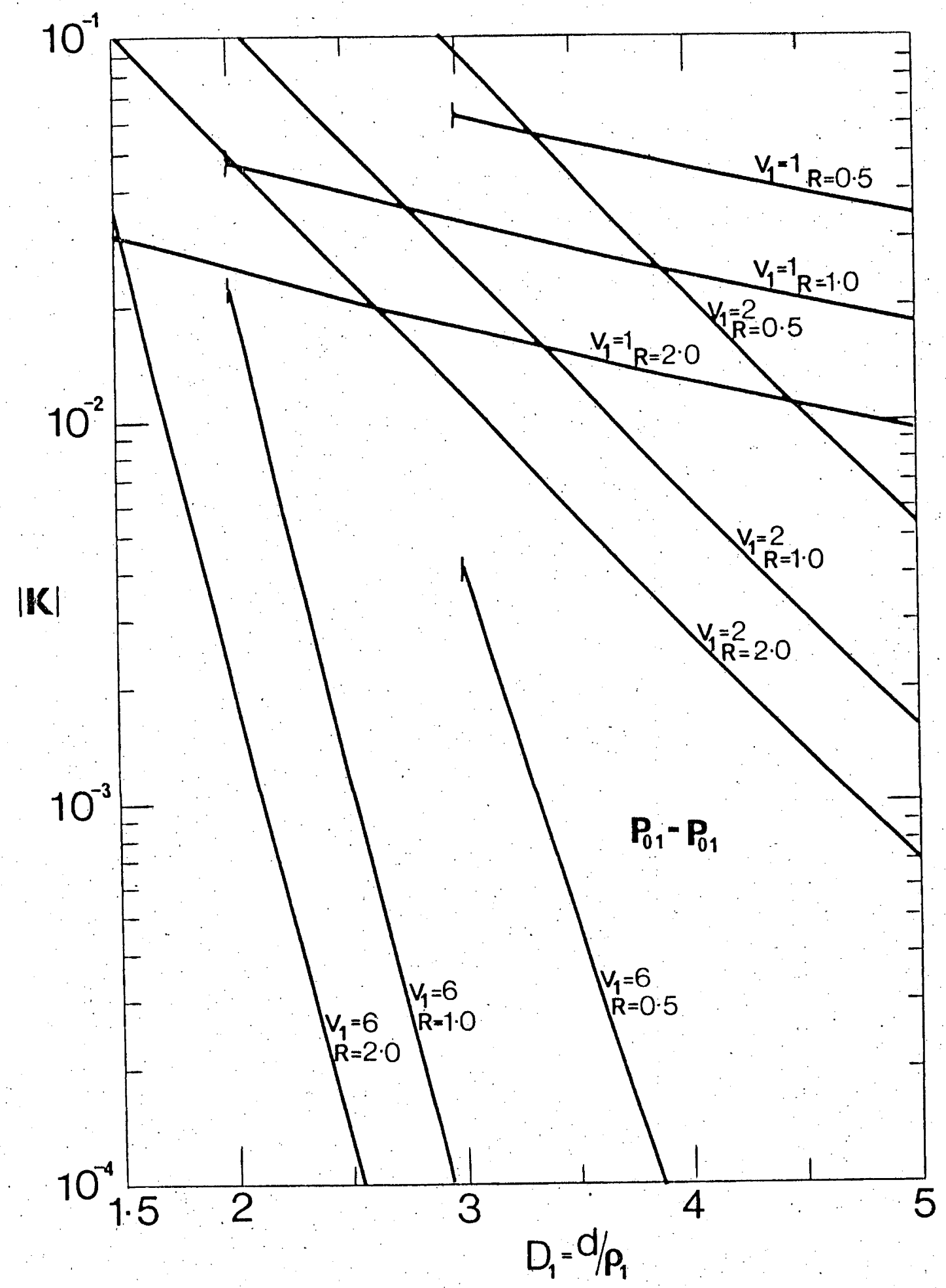

Fig. 3.20. The coupling constant $K$ as a function of $D_{1}=d / \rho_{1}, V_{1}$ and $R=\rho_{1} / \rho_{2}$ for $P_{01}-P_{0_{1}}$ coupling. The fibres are touching when $D_{1}=1+\rho_{2} / \rho_{1}$. 


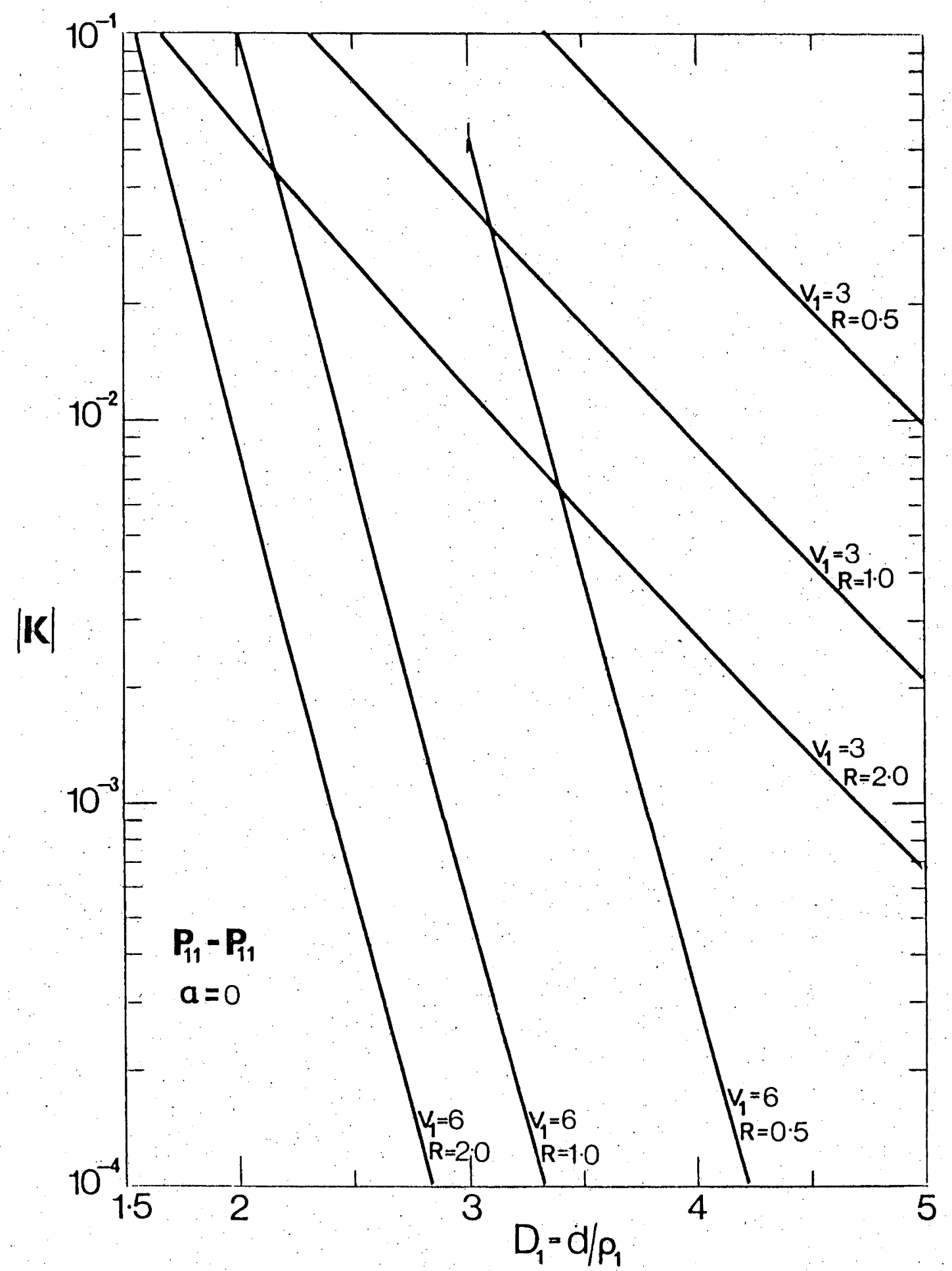

Fig. 3.21. As for $\mathrm{Fig}$ 3.20, but for $\mathrm{P}_{11}-\mathrm{P}_{11}$ coupling and $\alpha=0^{\circ}$. 


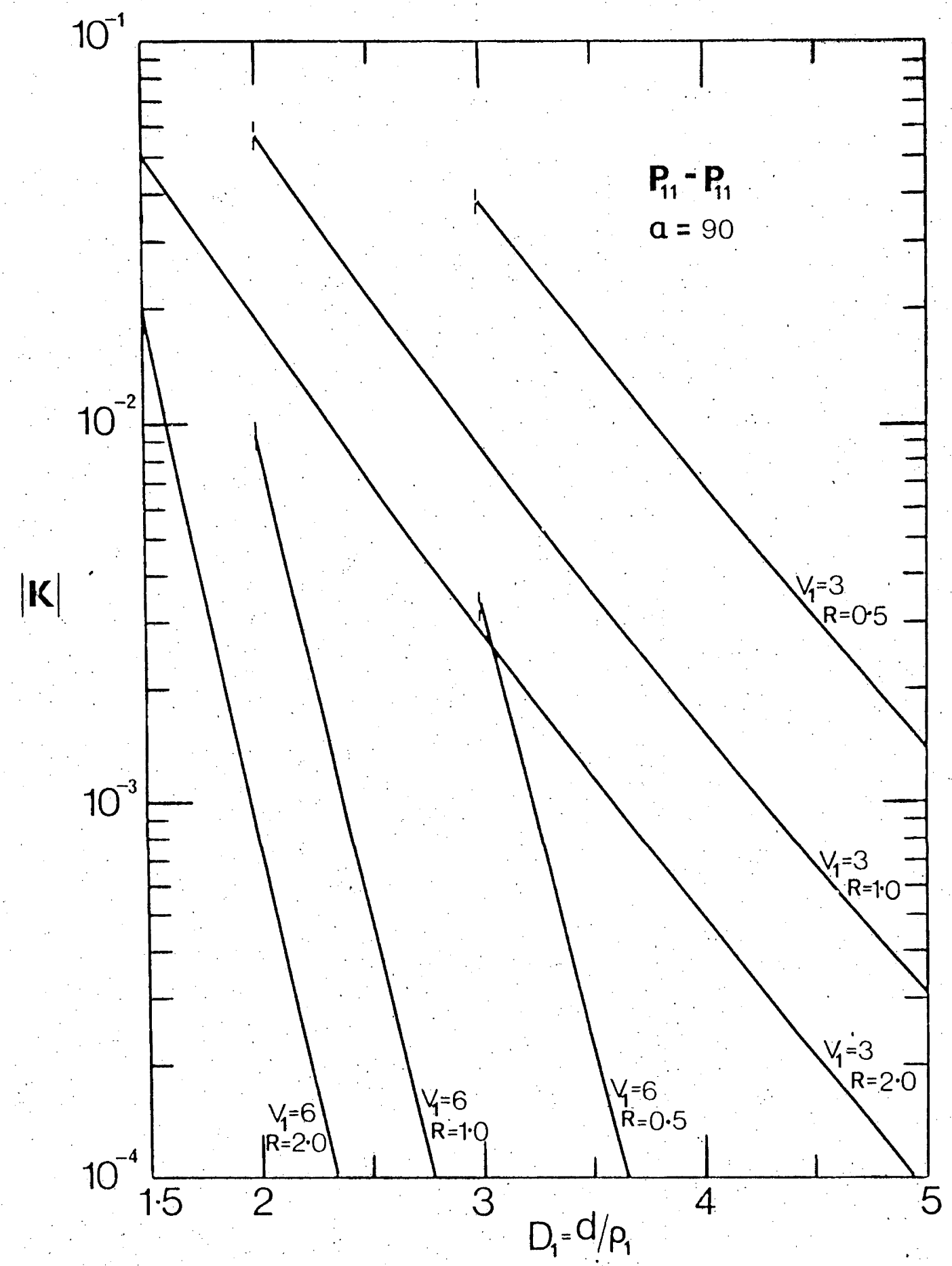

Fig. 3.22. As for Fig. 3.21, but with $\alpha=90^{\circ}$. 


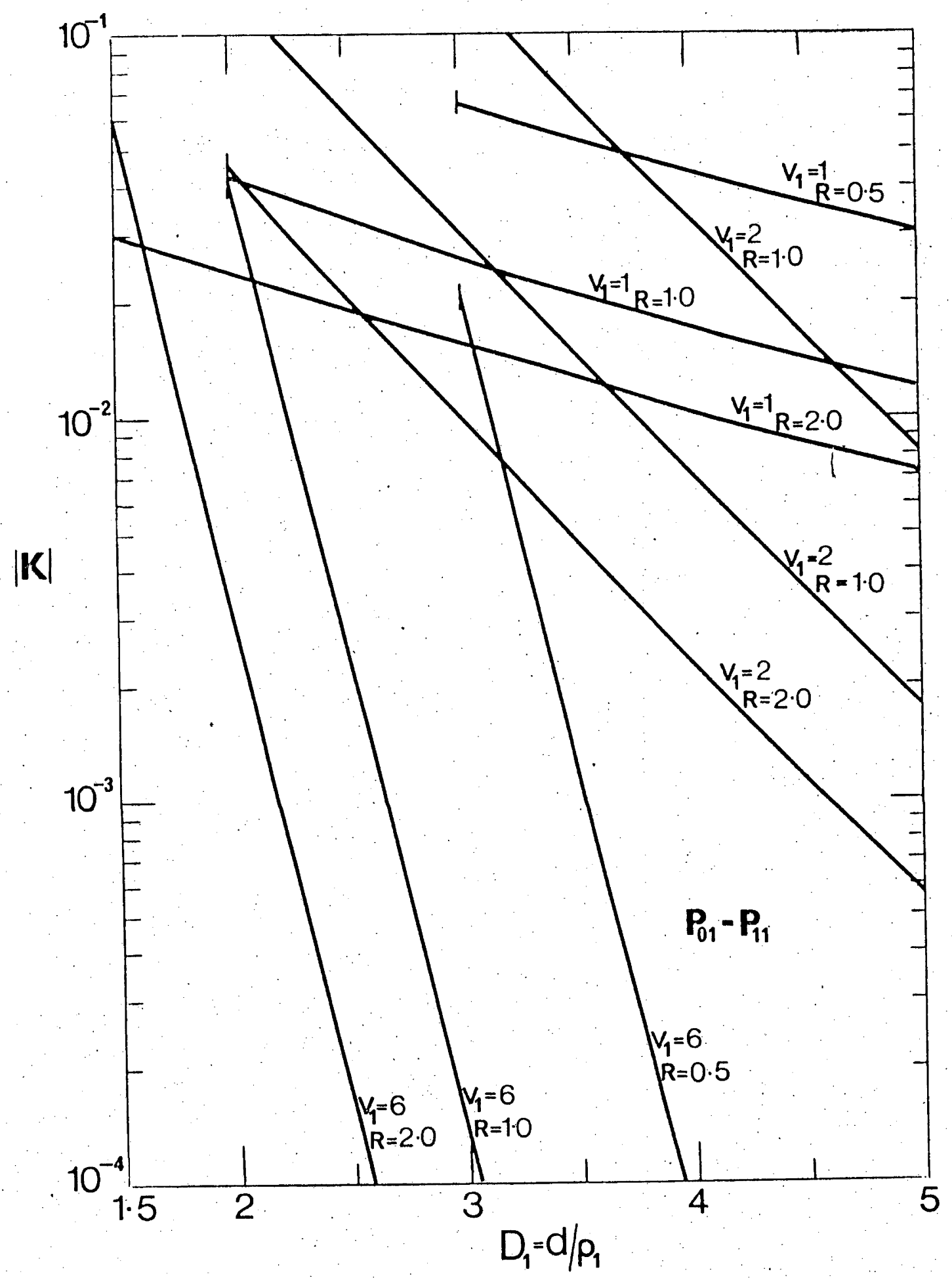

Fig. 3.23. As for Fig. 3.20, but for $\mathrm{P}_{01}-\mathrm{P}_{11}$ coupling and $\alpha=0^{\circ}$. For $\mathrm{P}_{01}-\mathrm{P}_{11}$ coupling at angle $\alpha$, $\kappa(\alpha)=\kappa(0) \cos \alpha$. 


$$
K=\frac{U^{2}}{V^{3}}\left[\frac{K_{2}(W D)-K_{0}(W D)}{K_{0}(W) K_{2}(W)}\right]
$$

Coupling between modes of either symmetry can then be found by varying the angle $\alpha$. Care must be taken in defining $\alpha$ when the coupling is between different modes.

The difference between the even-even and odd-odd coupling of $\mathrm{P}_{11}$ modes is analogous to the difference in the interaction energy of dipoles aligned along or perpendicular to the line joining the dipoles. Higher order modes correspond to higher multipoles. The coupling between the circularly symmetric $\mathrm{P}_{0 \mathrm{~m}}$ modes is of course independent of the angle $\alpha$ (corresponding to monopoles).

Qualitatively, the strength of coupling between two modes depends on the interaction or overlap of the modal fields. This can be seen from Figs. $3.24,3.25$; there is a greater overlap of the fields when $\alpha=0^{\circ}$ and therefore a larger coupling coefficient results. Similar reasoning partly explains why $P_{11}-P_{11}\left(\alpha=0^{\circ}\right)$ coupling is greater than $\mathrm{P}_{0_{1}}-\mathrm{P}_{01}$ coupling for given $\mathrm{V}_{1}$. The directional nature of the $P_{11}$ mode field leads to a greater interaction of the fields in the coupling region than for the circularly symmetric $P_{01}$ modes. The same effect occurs to a lesser extent in $\mathrm{P}_{0.1}-\mathrm{P}_{11}$ coupling. Coupling at an angle $\alpha$ for this case is given by

$$
\kappa(\alpha)=\kappa(0) \cos \alpha
$$

Here however, because of the opposite polarisation of the two halves of the $\mathrm{P}_{11}$ field, the $\mathrm{e} \cdot \mathrm{e}$ term in the coupling coefficient consists of two parts of opposite sign, and cancellation occurs. At $\alpha=90^{\circ}$ canceliation is exact and $k=0$. 

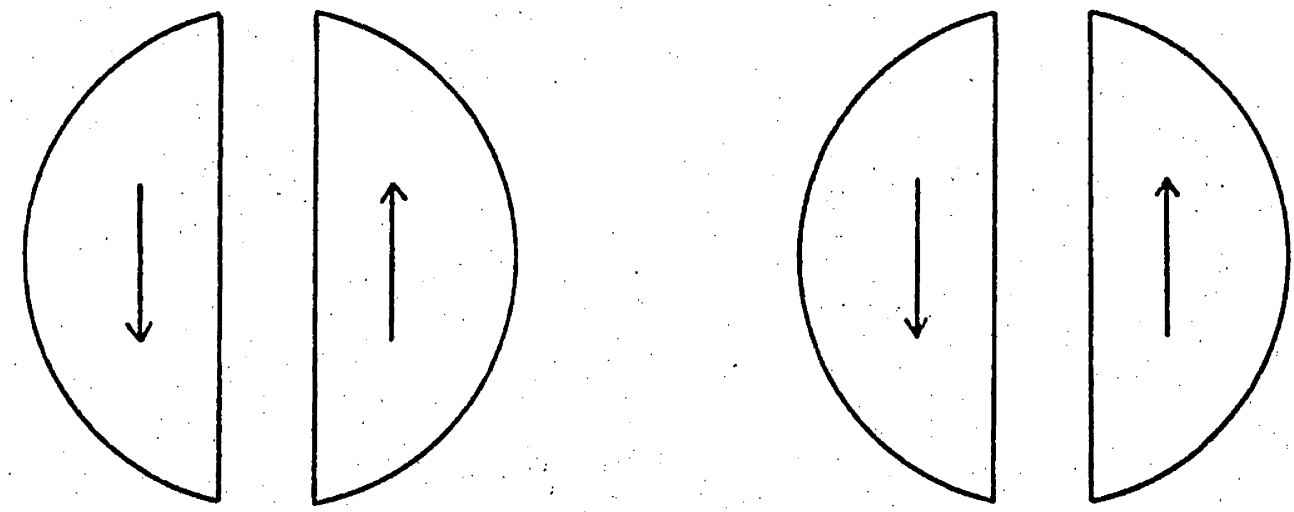

Fig. 3.24. Even-even coupling $\left(\alpha=0^{\circ}\right)$ between $P_{11}$ modes.
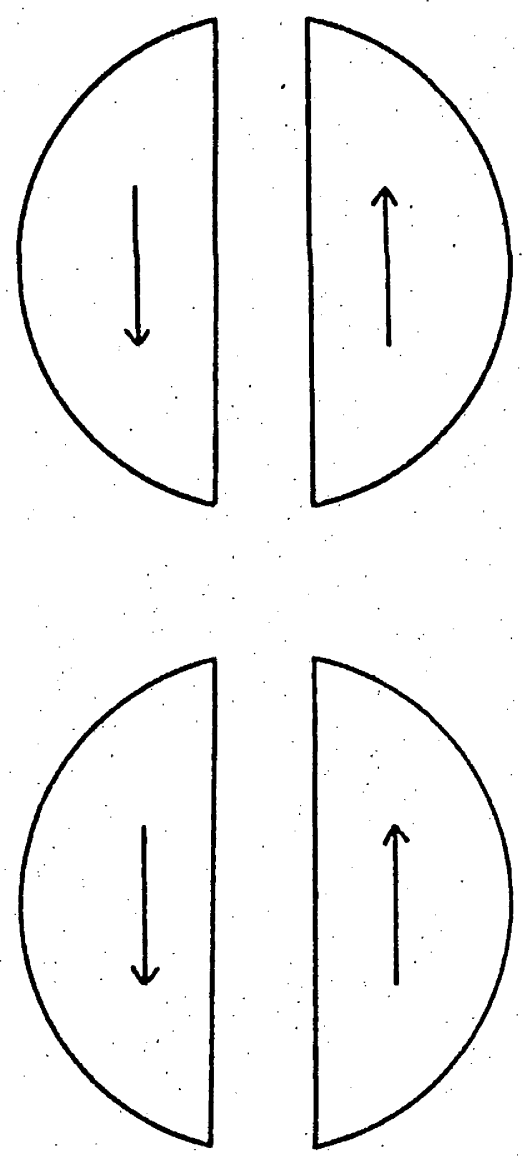

Fig. 3.25. Odd-odd coupling $\left(\alpha=90^{\circ}\right)$ between $P_{11}$ modes. 
The third factor to be taken into account is the fraction of the modal power that propagates outside the fibre in the evanescent field. The closer the mode is to cutoff (the smaller the V), the greater the fraction of power outside the fibre (Snyder, 1969a). Therefore by our qualitative argument, the coupling coefficient is in general larger, the smaller the $V$. Two trends arising from this effect are evident from the graphs: for given modes the coupling coefficient decreases with increasing $\mathrm{V}$, and for given $\mathrm{V}$, the coupling coefficient. increases with increasing mode order (ordered by cutoff). An apparent anomaly is that of $\mathrm{V}$ near the cutoff $(\mathrm{V}=0)$ of the $\mathrm{P}_{01}$ mode (Fig. 3.12), for which $K$ begins to decrease as $\mathrm{V}$ decreases. However this is explained by the fact that when $V$ is sufficiently close to cutoff, much of the modal power propagates outside the fibre and extends over a relatively large area, so that the field in the interaction region is actually smaller than for slightly larger values of V. As an indication of the spread of the field outside a fibre, we have plotted in Fig. 3.26 the penetration depth of the evanescent field of the $\mathrm{P}_{01}\left(\mathrm{HE}_{11}\right)$ mode as a function of $\mathrm{V}$. We define the penetration depth to be the radial distance (measured from the centre of the fibre) inside which is contained $90 \%$ of the power in the evanescent field. The fraction of the total modal power that is in the evanescent field is also shown. The curves demonstrate that for $\mathrm{V} \sim 1$, there is a large fraction of the modal power in the evanescent field and that this power is well spread-out. As $V$ is decreased below, 1 , the penetration depth increases rapidly. The dependence of $k$ on the separation $d$ of the two fibres is also due to the exponential fall-off of the fields with distance from the fibre. As the mode approaches cutoff, 


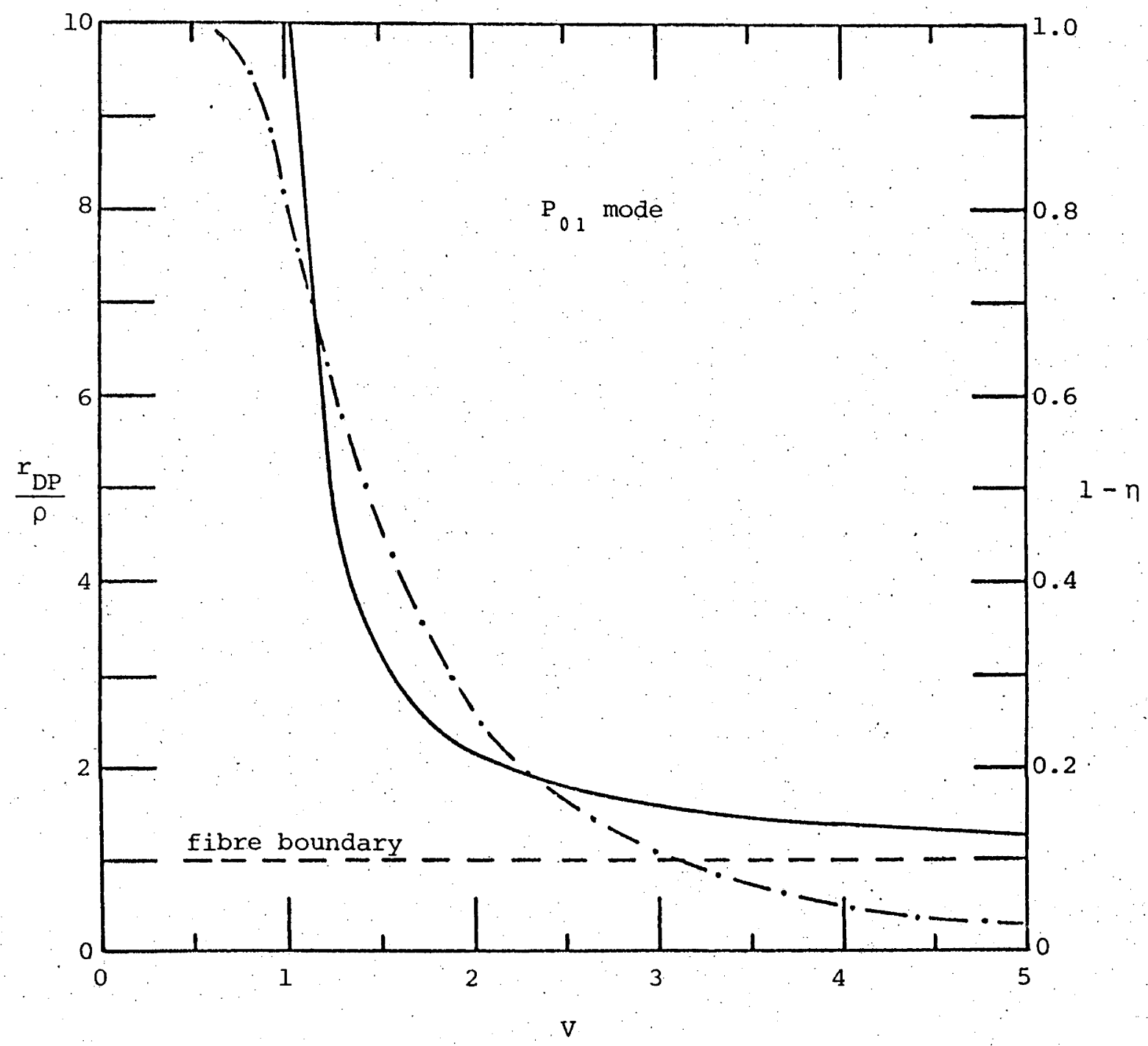

Fig. 3.26. The fraction $1-n$ of modal power that is outside the fibre (-.- - right-hand scale) and the normalised depth of penetration $r_{\mathrm{DP}} / \rho(-$ lefthand scale), as a function of $v$. See text for a definition of the depth of penetration. 
$K$ becomes less sensitive to changes in $\dot{d}$ because of the spread in the evanescent field.

A useful approximation for the coupling constant is given by using the asymptotic form of the Bessel function $\mathrm{K}_{\ell}$ for large argument

$$
K_{\ell}(z) \approx\left(\frac{\pi}{2 z}\right)^{\frac{3}{2}} e^{-z}
$$

giving for like modes

$$
\begin{aligned}
K & \approx \frac{\gamma_{\ell} U^{2}}{V^{3}} f(\alpha)\left(\frac{2 W}{\pi D}\right)^{\frac{1}{2}} e^{-W(D-2)} \\
& \approx \frac{\gamma_{\ell}^{2} U^{2}}{V^{5 / 2}} f(\alpha)\left(\frac{2}{\pi D}\right)^{\frac{1}{2}} e^{-V(D-2)}
\end{aligned}
$$

where $f(\alpha)=\cos ^{2} l \alpha\left(l_{1}=l_{2}\right)$. Equation (3.63) is a reasonable approximation when $W>1$, i.e. away from cutoff, and (3.64) is valid far from cutoff $\mathrm{W} \approx \mathrm{V} \gg \mathrm{U} . \gamma_{\ell}^{2}=1$ for $\mathrm{P}_{0 \mathrm{~m}}$ modes and 2 otherwise.

The power distribution in two neighbouring fibres when one mode of fibre 1 is initially excited, is given in terms of $k$ by

$$
\begin{aligned}
P_{1}(z) & =1-F \sin ^{2} \beta_{b} z \\
P_{2}(z) & =F \sin ^{2} \beta_{b} z \\
\beta_{b} & =\frac{\sqrt{\delta K}}{\rho \sqrt{F}} \\
F & =\left[1+\left(\frac{\rho \Delta \beta}{2 \sqrt{\delta} K}\right)^{2}\right]^{-1}
\end{aligned}
$$

The normalised transfer length $\mathrm{L}_{\mathrm{T}}\left(=\ell_{\mathrm{T}} / \rho\right)$ over which power $F$ is transferred from one fibre to the other is given by 


$$
\mathrm{L}_{\mathrm{T}}=\frac{\pi \sqrt{\mathrm{F}}}{2 \sqrt{\delta} \mathrm{K}}
$$

and the normalised beat length, the distance between successive maxima of power in one fibre, by $\mathrm{L}_{B}=2 \mathrm{~L}_{T}$. Total power transfer (to order $C / B$ ) takes place when $F=1$ i.e. $\Delta \beta=0$, but the total amount of power transferred is very sensitive to the difference in propagation constants. With $\delta=0.1$,

$$
\frac{\Delta \beta}{\beta}=\frac{\sqrt{\delta} \Delta \beta^{\prime}}{\rho \beta} \approx \frac{\delta \Delta \beta^{\prime}}{\mathrm{V}}=\frac{10 \Delta \beta^{\prime}}{\mathrm{V}}:
$$

A typical value of $\Delta \beta^{\prime}$ to reduce $F$ to 0.1 is about 0.1 when $v=2$ and the fibres are not touching (Figs. $3.7-3.10$ ). Half a percent difference is then all that is necessary to cut power transfer to $10 \%$. This corresponds in this case to a change in radius of about $7 \%$. With larger $\mathrm{V}$ (smaller $\mathrm{K}$ ), this difference is very much smaller. In Table 3.1 (page 82) we present two numerical examples of the effect of fibre parameters on the power transfer between two fibres.

As a further check on the accuracy of our results, particularly on the original assumption that the fields of a two-fibre system are superpositions of the individual-fibre modes (Section 2.3.2), we compare our results with those of Wijngaard (1973): He solves the full normal-mode boundary-value problem numerically by a method that is exact in principle, although some approximations are necessary to carry out the calculations. Wijngaard also takes the $\delta \rightarrow 0$ limit of his calculations and finds agreement with Vanclooster and Phariseau (1970, 1970a); Snyder (1972) has shown the equivalence of the 
(1)

\begin{tabular}{|l|l|l|l|l|}
\hline$D$ & $K$ & $l_{t} / \rho_{1}$ & $\frac{\Delta \beta}{\beta_{1}}$ & $\Delta\left(\rho_{1} / \rho_{2}\right) \%$ \\
\hline $2.94(t)$ & -0.14 & 49 & 4 & 54 \\
3 & -0.13 & 54 & 2 & 44 \\
4 & -0.031 & 226 & 0.7 & 8 \\
6 & -0.0019 & 3725 & 0.04 & 0.4 \\
\hline
\end{tabular}

(2)

\begin{tabular}{|c|c|c|c|c|}
\hline $\mathrm{D}$ & $\mathrm{K}$ & $\ell_{\mathrm{t}} / \rho_{1}$ & $\frac{\Delta \beta}{\beta_{1}}$ & $\Delta\left(\delta_{2} / \delta_{1}\right) \%$ \\
\hline $2(t)$ & -0.084 & 173 & 0.13 & 4 \\
3 & -0.002 & 7300 & $3 \times 10^{-3}$ & 0.3 \\
4 & $-5.1 \times 10^{-5}$ & $2.8 \times 10^{5}$ & $<10^{-3}$ & $<10^{-2}$ \\
6 & $-3.6 \times 10^{-8}$ & $4 \times 10^{8}$ & $<10^{-4}$ & $<10^{-3}$ \\
\hline
\end{tabular}

\section{Table 3.1}

Table 3.1 Effect of fibre parameters on power transfer. $\Delta \beta_{1} \beta_{1}$ gives the percent difference in the propagation constants necessary to reduce the maximum power transfer $F$ to $0.1 . \Delta\left(\rho_{1} / \rho_{2}\right)$ or $\Delta\left(\delta_{2} / \delta_{1}\right)$ are the corresponding variations in the fibre parameters, $\ell_{T}$ is the distance for total power transfer and $t$ indicates touching fibres. The two examples are

(1) $\mathrm{P}_{01}-\mathrm{P}_{11}$ coupling with $\mathrm{V}_{1}=2, \delta_{1}=\delta_{2}=0.1$. For $\beta_{1}=\beta_{2}, V_{2}=3.89$ and $\rho_{1} / \rho_{2}=0.515 . \alpha=0$.

(2) $\mathrm{P}_{01}-\mathrm{P}_{11}$ coupling with $\mathrm{V}_{1}=4, \rho_{1}=\rho_{2}$ and $\delta_{1}=0.01$. For $\beta_{1}=\beta_{2}, v_{2}=4.7$ and $\delta_{2} / \delta_{1}=1.374 . \alpha=0$. 
formulation given here with that of Vanclooster and Phariseau. To show agreement with other formulations we also compare our results with those of Burke (1967), who uses the earlier theories of Jones (1965) and Bracey et al (1959) to calculate a parameter $G(V)$ [in Burke's notation $C(V)$ ] which is related to $K$ by

$$
G(V)=\frac{n_{1} V}{4 K}
$$

and to Wijngaard's parameter $\gamma$ by $G(V)=n_{1} \gamma$. These two sets of results, together with ours calculated from (3.71), are presented in Fig. 3.27 for $P_{01}$ modes on identical fibres with $n_{1}=1.53$, for several values of $d / \rho$. Burke's calculations use the asymptotic form for the Bessel functions which give inaccurate answers below the negatively-sloped dashed line. Above this line, our results agree substantially with his. Wijngaard only lists numerical results for touching fibres, for which we could expect coupled-mode theory to be inaccurate. However his results indicate that even for touching fibres with relatively low values of $\mathrm{V}$, coupled-mode theory provides a good approximation. The error at $v=1.6$ is only about $4 \%$. Below the curve $C_{T^{\prime}}$, taken from Wijngaard (1973), the propagation constants $\gamma$ of the normal modes in the coupled-mode approximation $(\beta \pm C$ - Section 2.5), are outside the range $\mathrm{k}_{2}<\gamma<\mathrm{k}_{1}$, where $\mathrm{k}_{1,2}$ are the plane-wave propagation constants in the core and cladding media respectively. The curves in Fig. 3.27 demonstrate the equivalence of the various methods in determining the transfer length for neighbouring optical fibres.

A possible application of fibre coupling is the use of pairs of fibres as a switching device (Kapany et al, 1965). By altering the difference $\Delta \beta$ in the propagation constants of the two fibres, e.g. by changing the radius, the transfer length may be 


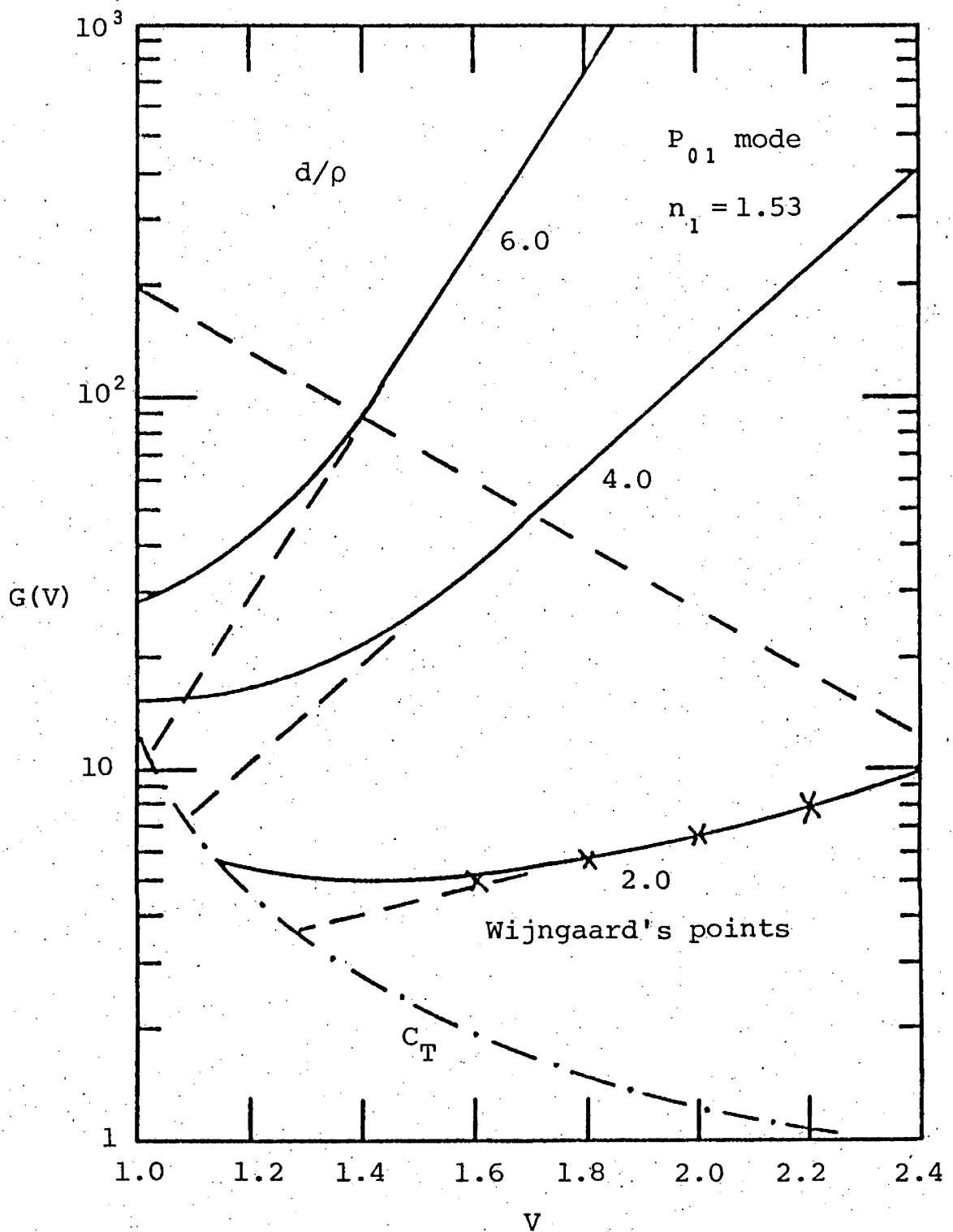

Fig. 3.27. Burke's parameter $G(V)=\frac{n_{1} V}{4|K|}$ calculated from (3.71) (-). Burke's results $(--)$ are inaccurate below the negatively sloped dashed line (see text) and are almost identical to our results above it. Also shown are values taken from the full normal-mode calculations of wijngaard. 
tuned to required values, hopefully without sacrificing too much power transfer (Reinhart and Wilson, 1974). In practice however this is difficult because the transfer length is less sensitive to changes in. $\Delta \beta$ than is the total power transfer $F$. This imposes very strict requirements on the tolerances in the manufacture of the fibre pairs. A more general and easily realisable application of fibre coupling is the use of a neighbouring fibre to sample a small part of the main signal (Kuwahara et al, 1975).

Comparison of theory with experiment is difficult because of the small size of fibre necessary for single-mode operation and the exact tolerance required in fabrication of fibres to duplicate the theoretical conditions. Experiments have been performed however and Bracey et al (1959), for example, find good agreement between their theory and experimental results with $\delta=0.61$. Kapany and Burke (1972 - Chapter 7) provide detailed experimental data and discuss at length their results and the limitations of the experiment. They conclude that there is reasonable agreement between experiment and theory.

The only more recent experimental results on power transfer are provided by Kuwahara et al (1975), who use a multimode fibre $(\mathrm{V}=25.58)$ : They assume only $\mathrm{HE}_{1 \mathrm{~m}}$ modes are excited (up to $\left.\mathrm{HE}_{18}\right)$, measure the power distribution in the modes of the excited fibre and calculate the amount of power transferred. They conclude that the observed power transfer is larger than that predicted by coupling theory. However they give no experimental results or measurements of the power distribution and give coupling coefficients which differ from those calculated using the theory presented here. Given the 
correct coupling coefficients (or even theirs), it seems that the only significant contribution to the coupling will come from the $\mathrm{HE}_{1 \mathrm{~g}}$ mode, which is presumably excited with the least power. Without a knowledge of the accuracy of the measurement of the power distribution in the modes and of the other assumptions, no conclusions can be drawn.

\subsection{POWER TRANSFER IN OPTICAL-FIBRE ARRAYS}

Of particular interest is coupling in fibre arrays which occur in fibre optics (Kapany, 1967), optical communications and in both vertebrate and invertebrate eyes. For example in the human retina the receptors are closely packed, with each receptor in the center of a hexagonal array of nearest neighbours.

Snyder (1972) shows how the coupled-mode equations for $\mathrm{HE}_{11}$ modes on identical-fibre arrays reduce to a two-mode form. In this section we generalise the procedure to higher-order modes and include cross-mode coupling as well. The arrays can contain any number of fibres, but in general ( $P_{0 m}$ modes excepted) the more fibres, the larger the number of equations to be solved. Because it is impossible to take account of arbitrary combinations of mode and array symmetries, we illustrate our approach with several examples.

Consider coupling of $\mathrm{P}_{11}$ modes (Fig. 3.2) on the array shown in Fig. 3.28. If the field of each fibre is small in the region of the other fibres, then $\left|C_{i j}\right| \ll\left|\beta_{i}\right|,\left|\beta_{j}\right|$ and the considerations of Chapter 2 apply. Coupling is described by seven coupled equations of the form (2.20). The form of the equations can be simplified if only nearestneighbour coupling is taken into account. Each fibre couples only with its two nearest neighbours and the centre fibre. Then iffibres 1-4 are 

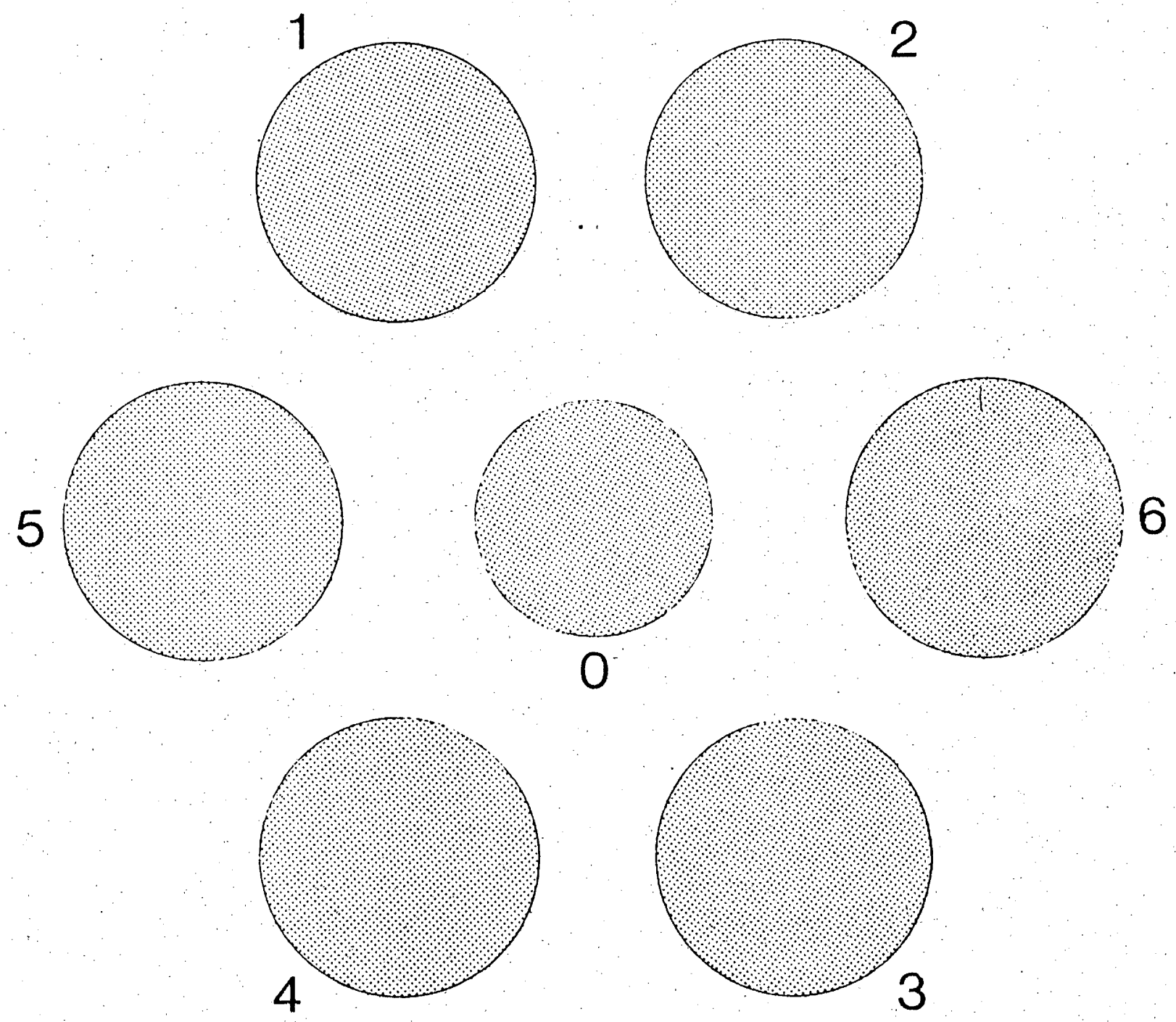

Fig. 3.28. Hexagonal array of fibres. 
identical and fibres 5, 6 are identical, the equations reduce to three equations

$$
\begin{aligned}
& \frac{d A_{1}}{d z}+i \beta_{1}^{A} A_{1}=i C_{12}^{A} A_{2}+i C_{13}^{A} A_{3} \\
& \frac{d A_{2}}{d z}+i \beta_{2}^{A} A_{2}=i C_{21}^{A} A_{1}+i C_{23}^{A} A_{3} \\
& \frac{d A_{3}}{d z}+i \beta_{3}^{A} A_{3}=i C_{31}^{A} A_{1}+i C_{32}^{A} A_{2}
\end{aligned}
$$

where the superscript A refers to an array parameter, and

$$
\begin{aligned}
A_{1}(z) & =a_{0}(z) \\
A_{2}(z) & =\frac{1}{2} \sum_{j=1}^{4} a_{j}(z) \\
A_{3}(z) & =\frac{1}{\sqrt{2}}\left[a_{5}(z)+a_{6}(z)\right] \\
\beta_{1}^{A} & =\beta_{0} \\
\beta_{2}^{A} & =\beta_{1}-C_{12} \\
\beta_{3}^{A} & =B_{5} \\
C_{12}^{A} & =2 C_{01} \\
C_{13}^{A} & =\sqrt{2} C_{05} \\
C_{23}^{A} & =\sqrt{2} C_{15}
\end{aligned}
$$

The quantities to the right of the $=$ signs in $(3.75)-(3.83)$ are individual-fibre variables, e.g. $C_{05}$ is the coupling coefficient between $P_{11}$ modes on fibre 0 and fibre 5. If the $B$ 's of the individual fibres $i, j$ are equal, then $C_{i j}=C_{j i}$ (see section 3.5.3). Coupling between all fibres rather than only nearest neighbours could have been included, 
but the resulting system of equations is more complicated (Snyder, 1972). In most cases we can neglect all but nearest neighbour coupling.

The use of (3.72) - (3.83) can be generalised. Provided that the fibres with each symmetry grouping $(0,1-4,5-6)$ are identical, the equations are valid; each symmetry group can however, differ from the others in both fibre properties and propagating modes (provided that this does not destroy the symmetry). Only the individual-fibre coupling coefficients $\left(C_{i j}\right)$ and propagation constants will change. This allows us to treat, for example, cross-mode coupling on this array with the same equations. With the appropriate coupling coefficients, the above equations can describe coupling between a $P_{01}$ mode on the centre fibre and $P_{11}$ modes on the surrounding fibres $1-6$. The point is that each set of equations such as (3.72)-(3.83) can describe a variety of situations, provided that the appropriate symmetry grouping is maintained. Breakdown of this symmetry will usually mean more equations and a resultant loss of simplicity.

For each particular combination of fibres and modes there is a minimum number of equations needed to describe power transfer in the array; the minimum number of equations applies when there is maximum symmetry and is the same as the number of different symmetry groups. In Table 3.2 we give the minimum number of equations necessary to describe coupling between like modes on arrays with from two to ten surrounding fibres. 


\begin{tabular}{|c|c|c|c|c|}
\hline $\begin{array}{r}\text { Number of } \\
\text { surrounding fibres }\end{array}$ & $\mathrm{P}_{0 \mathrm{~m}}$ & $\mathrm{P}_{1 \mathrm{~m}}$ & $\mathrm{P}_{2 \mathrm{~m}}$ & $\mathrm{P}_{3 \mathrm{~m}}$ \\
\hline 2 & 2 & 2 & 2 & 2 \\
3 & 2 & 3 & 3 & 2 \\
$4 \mathrm{a}$ & 2 & 3 & 2 & 3 \\
$4 \mathrm{~b}$ & 2 & 2 & 2 & 2 \\
5 & 2 & 4 & 4 & 4 \\
6 & 2 & 3 & 3 & 2 \\
7 & 2 & 5 & 5 & 5 \\
$8 \mathrm{a}$ & 2 & 4 & 3 & 4 \\
$8 \mathrm{~b}$ & 2 & 3 & 2 & 3 \\
9 & 2 & 6 & 6 & 3 \\
10 & 2 & 4 & 4 & 4 \\
\hline
\end{tabular}

a. Surrounding fibres on the $x$ and $y$ axes of the central fibre.

b. No surrounding fibres on the $x$ or $y$ axes of the central fibre.

\section{Table 3.2}

As a further example of how symmetry can reduce the number of equations, consider coupling between $P_{31}$ modes (Fig. 3.2) on the array of Fig. 3.28, with the six surrounding fibres identical (the centre fibre can be different). Because the surrounding fibres are symmetricaliy placed, this system is described by two equations, with

$$
\begin{aligned}
& A_{1}(z)=a_{0}(z) \\
& A_{2}(z)=\frac{1}{\sqrt{6}} \sum_{j=1}^{6} a_{j}(z)
\end{aligned}
$$

and

$$
\begin{aligned}
& \dot{\beta}_{1}^{A}=\beta_{0} \\
& \beta_{2}^{A}=\beta_{1}-2 c_{12} \\
& c_{12}^{A}=\sqrt{6} c_{01} \approx c_{21}^{A}
\end{aligned}
$$


This result is the same as that found for $P_{01}$ coupling by Snyder (1972), and the discussion there is relevant to this situation.

Having found the minimum number of coupled-mode equations, we proceed exactly as for the two-fibre case. Equations with approximately equal $\beta_{i}^{A}$ can be solved separately or decoupled from the other equations. If, in the first example above, only two of the three $\beta_{i}^{A}$ are approximately equal, $\beta_{1}^{A} \approx \beta_{2}^{A} \approx \beta_{3}^{A}$ as given by $(3.78)-$ (3.80), then coupling between fibres $0-4$ and 5,6 can be neglected, leaving only two equations to be solved; fibres 5 and 6 are effectively isolated. The shift in propagation constants e.g. (3.87) allows for the possibility of coupling between modes that would not couple in a two-fibre system, a fact which must be taken into account when formulating the array equations.

In the two-equation case, the maximum power transferred from the centre fibre to the surrounding fibres is given by $F=\left[1+\left(\frac{\Delta \beta^{A}}{2 C_{12}^{A}}\right)^{2}\right]^{-1}$ as before. However, because the array propagation constants are not necessarily equal to the fibre propagation constants, in general total power transfer will not take place in an array of identical fibres. This is discussed in greater detail (for $\mathrm{HE}_{11}$ modes) by Snyder (1972). We reproduce in Table 3.3 his results showing the percentage difference in radius between the centre fibre and the surrounding fibres necessary to achieve total power transfer in arrays of from 2 to 11 fibres. 


\begin{tabular}{|c|c|c|c|}
\hline $\mathrm{n}$ & $\mathrm{F}\left(\frac{8}{8}\right)$ & $\frac{\Delta \rho}{\rho}\left(\frac{8}{0}\right)$ & $\frac{\ell_{\mathrm{T}}}{\rho} \times 10^{-3}$ \\
\hline 1 & 100 & - & 8.7 \\
2 & 100 & - & 6.1 \\
3 & 100 & - & 5.0 \\
4 & 99.96 & $2.2 \times 10^{-3}$ & 4.3 \\
5 & 99.02 & $1.2 \times 10^{-2}$ & 3.9 \\
6 & 85.71 & 0.55 & 3.3 \\
7 & 44.75 & 1.6 & 2.2 \\
8 & 18.18 & 3.4 & 1.3 \\
9 & 6.17 & 6.5 & 0.75 \\
10 & 2.44 & 11.0 & 0.43 \\
\hline
\end{tabular}

Table 3.3

Table 3.3 The percent maximum power transfer between the centre and surrounding fibres of a regular array of identical fibres. The centre fibre is excited in the $P_{01}$ mode and $n$ is the number of surrounding fibres. $\Delta \rho / \rho$ is the increase in the radius of the central fibre required for total power transfer and $l_{T}$ is the length required for the transfer. $V=2.5, \delta=0.01$ and $d / \rho=4$ where $d$ is the distance between the centres of the central and surrounding fibres. From Snyder (1972).

In Fig. 3.29 we show the power transfer in a hexagonal array of identical fibres as a function of $\mathrm{Cz}$ and compare it with power transfer between two fibres. The central fibre of the array is excited in the $P_{01}$ mode. The six surrounding fibres of the array represent a greater perturbation than the one fibre of the two-fibre system and 


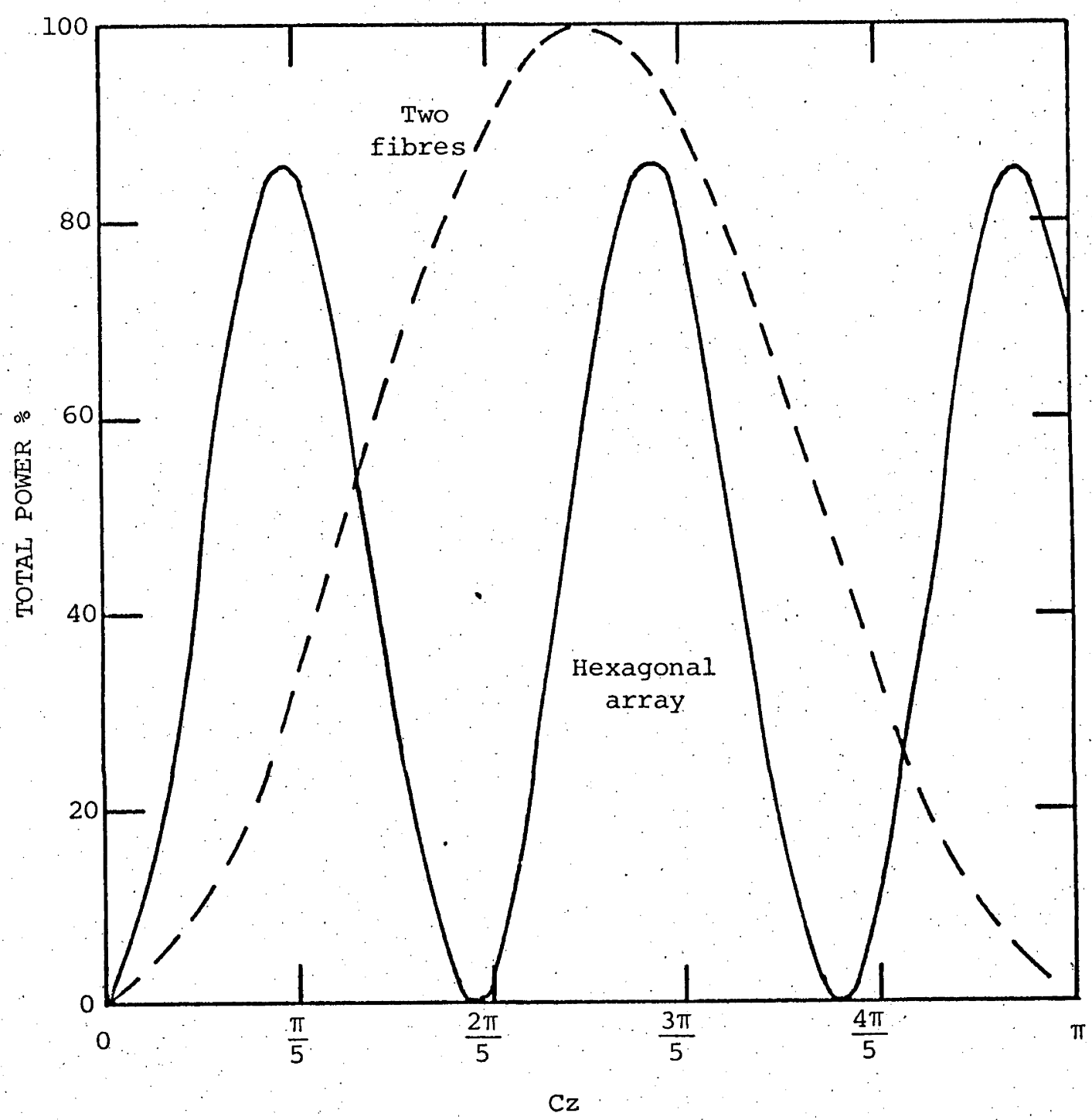

Fig. 3.29. Total power in the six surrounding fibres of an hexagonal array of identical fibres, when the centre fibre is excited in the $P_{0.1}$ mode, as a function of $\mathrm{Cz} . \mathrm{C}$ is the coupling coefficient between any two of the fibres. Also shown is the power in the unilluminated fibre of a two-fibre system with the same separation as in the array (- - $\rightarrow$ ) 
therefore the rate of power transfer in the array is faster. However incomplete power transfer takes place because the six surrounding fibres do not appear as an identical fibre to the central fibre.

Wijngaard (1974) has extended his numerical calculations on the normal modes of two fibres (Wijngaard; 1973) to an infinite hexagonal array of fibres. In the limit $\delta \rightarrow 0$ his formulation reduces to the one presented here. Other works include that of Jones (1965), who analyses an infinite linear array of fibres, and Vanclooster and Phariseau (1970b) who discuss diffraction of a plane wave by a fibre bundle:

\section{APPENDIX VALIDITY OF $\mathrm{P}_{\ell \mathrm{m}}$ MODES FOR COUPLING BETWEEN TWO FIBRES}

In Section 3.4 we examine the validity of the $P_{\ell m}$ modes for a single waveguide and obtain upper limits on the length of waveguide for which the modes are valid. In Section 3.5 we find that the modes which interact most strongly in a coupled-mode theory are those with approximately equal propagation constants. As the basis of using the $\mathrm{P}$ modes is the fact that the component $\mathrm{HE}$ and $\mathrm{EH}$ modes have the same propagation constant in the limit $\delta \rightarrow 0$, we might expect that the component modes will be coupled by the presence of a neighbouring waveguide, thereby causing a further breakdown of the $P$ modes. This of course does not apply to the $\mathrm{P}_{\text {om }}$ modes, which remain valid for any length waveguide and irrespective of whether or not there are neighbouring waveguides.

To examine the interaction of the component $\mathrm{HE}_{\ell+1, \mathrm{~m}}$ and $\mathrm{EH}_{\ell-1, \mathrm{~m}}$ modes of the $\mathrm{P}_{\ell \mathrm{m}}$ mode, we calculate the coupling coefficient, the power transfer between the component modes and the length of waveguide over which the two modes exchange power in the presence of a 
neighbouring identical waveguide. These parameters are functions of $v, \delta$ and the normalised separation $D\left(=d_{s} / \rho\right)$ of the waveguides. The coupling coefficient is calculated as in Section 3.5 i.e.

$$
C_{W}=-\frac{\omega}{2}\left(n_{2}^{2}-n^{2}\right) \int_{A_{2}} e_{-H E} \cdot-_{E H} d A,
$$

where the fields to be used are the $R \geqslant 1$ fields given by $(3.14)$ and (3.9). The subscript $w$ is used to distinguish the quantities here from those of previous sections. Defining a coupling constant $\kappa_{W}$ as before, and carrying out the integrations leads to

$$
\begin{aligned}
& K_{W}=\frac{U^{2}}{V} \frac{1}{K_{\ell+1}(W) K_{\ell-1}(W)} \\
& \times \sum_{j=0}^{\infty} K_{\ell+j}(W D) K_{\ell-j}(W D)\left[I_{j}^{2}(W)+I_{j-1}(W) I_{j+1}(W)\right],
\end{aligned}
$$

where the prime on the summation indicates that the $j=0$ term is to be multiplied by 0.5. Only the first few terms of the sum need be calculated, because it converges very rapidly.

The maximum power transfer $\mathrm{F}_{\mathrm{W}}$ between the $\mathrm{HE}$ and the $\mathrm{EH}$ mode is given by

$$
F_{W}=\left[1+\left(\frac{\rho \beta_{d}}{2 \sqrt{\delta k}}\right)^{2}\right]^{-1},
$$

where $\beta_{\mathrm{d}}$ is the difference between the propagation constants of the $\mathrm{HE}_{\ell+1, \mathrm{~m}}$ and $\mathrm{EH}_{\ell-1, \mathrm{~m}}$ modes, used to calculate $\overline{\mathrm{L}}$ in $(3.31)$, and found by solving the full $(\delta \neq 0)$ eigenvalue equation. The $\mathrm{HE}$ and $\mathrm{EH}$ modes exchange power $F_{W}$ in a length $\ell_{W}$ given by

$$
L_{W}=\frac{\ell}{\rho}=\frac{\pi \sqrt{F_{W}}}{2 \sqrt{\delta_{K_{W}}}} .
$$

In Figs. $3.30-3.32$ the quantities $\mathrm{I}_{\mathrm{W}}$ and $\mathrm{F}_{\mathrm{W}}$ are plotted as functions of 
$\mathrm{D}, \mathrm{V}$ and $\delta$ for $\mathrm{HE}_{21}-\mathrm{TM}_{01}$ coupling, together with the transfer length $\mathrm{L}_{\mathrm{T}}$ for power transfer between $\mathrm{P}_{11}$ modes on the two waveguides.

Also included in these figures is the upper limit $\bar{L}$ on the length of validity of the $\mathrm{P}$ modes. The curves show that when the waveguides are not touching or the modes too close to cutoff, the transfer length $L_{T}$ is generally much less than the length $L_{W}$ or $F_{W} \ll 1$, so that the $P_{11}$ mode propagates as an entity over this length and can be used to describe coupling. This is consistent with the findings of wijngaard (1973) who shows that when two waveguides are not touching, the normal modes of the two-waveguide system are approximately superpositions of the $\mathrm{HE}$ or $\mathrm{EH}$ modes of the individual waveguides. By taking the sum and difference of these normal modes, we recover $P$ mode coupling.

If the waveguides are touching or the modes close to cutoff, Figs.3.30-3.32 show that $\mathrm{I}_{\mathrm{W}}$ and $\mathrm{L}_{\mathrm{T}}$ are approximately equal (and $\mathrm{F}_{\mathrm{W}} \approx 1$ ). Therefore the coupling between modes on neighbouring waveguides and coupling between modes on the same waveguide are of the same magnitude, necessitating the solution of a 4-mode coupling problem (except for $\mathrm{P}_{01}$ modes). The normal mode approach of wijngaard shows that for this situation, the normal modes are superpositions of $\mathrm{HE}$ and $\mathrm{EH}$ modes (i.e. $\mathrm{HE}_{21}$ and $\mathrm{TM}_{01}, \mathrm{TE}_{01}$ on each waveguide). Because there are two such superpositions (sum and difference) with different phase velocities they beat together, giving the appearance of the $\mathrm{HE}$ and $\mathrm{EH}$ modes on each waveguide exchanging power or coupling, as well as coupling between the waveguides. This corresponds to our findings.

We conclude that for non-touching waveguides and modes not too close to cutoff, the $\mathrm{P}_{\ell m}$ modes may be used to treat coupling between neighbouring waveguides. The limitation on the length of the waveguide 


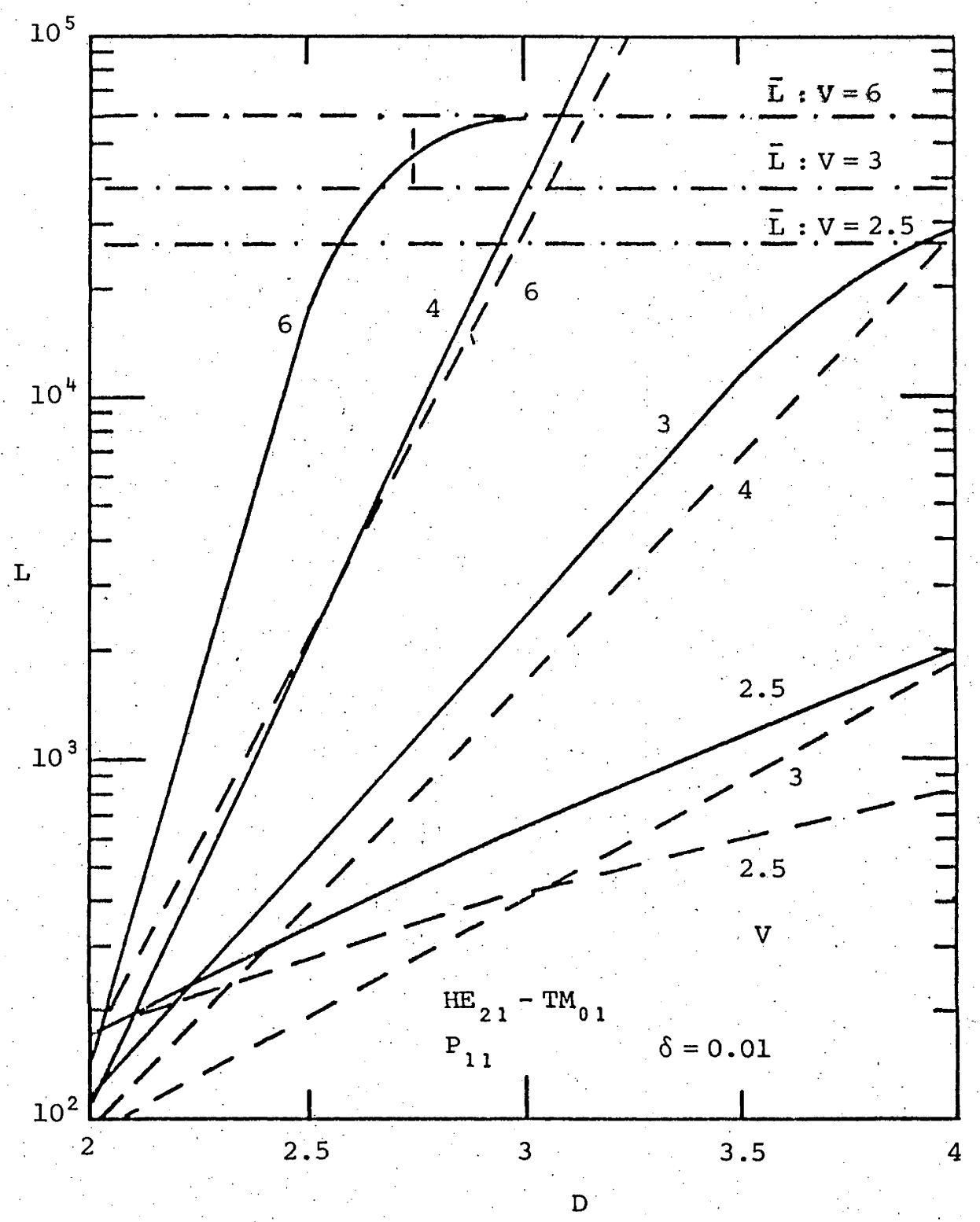

Fig. 3.30. The normalised length $L=\ell / \rho$ of fibre for total power transfer as a function of normalised separation $D=\frac{d}{\rho}$.

(a) $L_{W}$ the length for power transfer between the $\mathrm{HE}_{21}$ and $\mathrm{TM}_{01}$ modes on one fibre $(\longrightarrow$ ). The vertical bars indicate where $\mathrm{F}_{\mathrm{W}}=0.1$. For larger values of $\mathrm{D}, \mathrm{F}_{\mathrm{W}}<0.1$.

(b) $I_{T}$ the length for power transfer between $\mathrm{P}_{11}$ modes $\left(\mathrm{HE}_{21} \pm \mathrm{TM}_{01}\right)$ on the two fibres $(--\longrightarrow)$.

(c) $\bar{L}$ the length of a fibre in isolation for which the $P_{11}$ modes are valid $(-.--)$ ). $\overline{\mathrm{L}}$ for $\mathrm{V}=4$ is $2.5 \times 10^{5}$. 


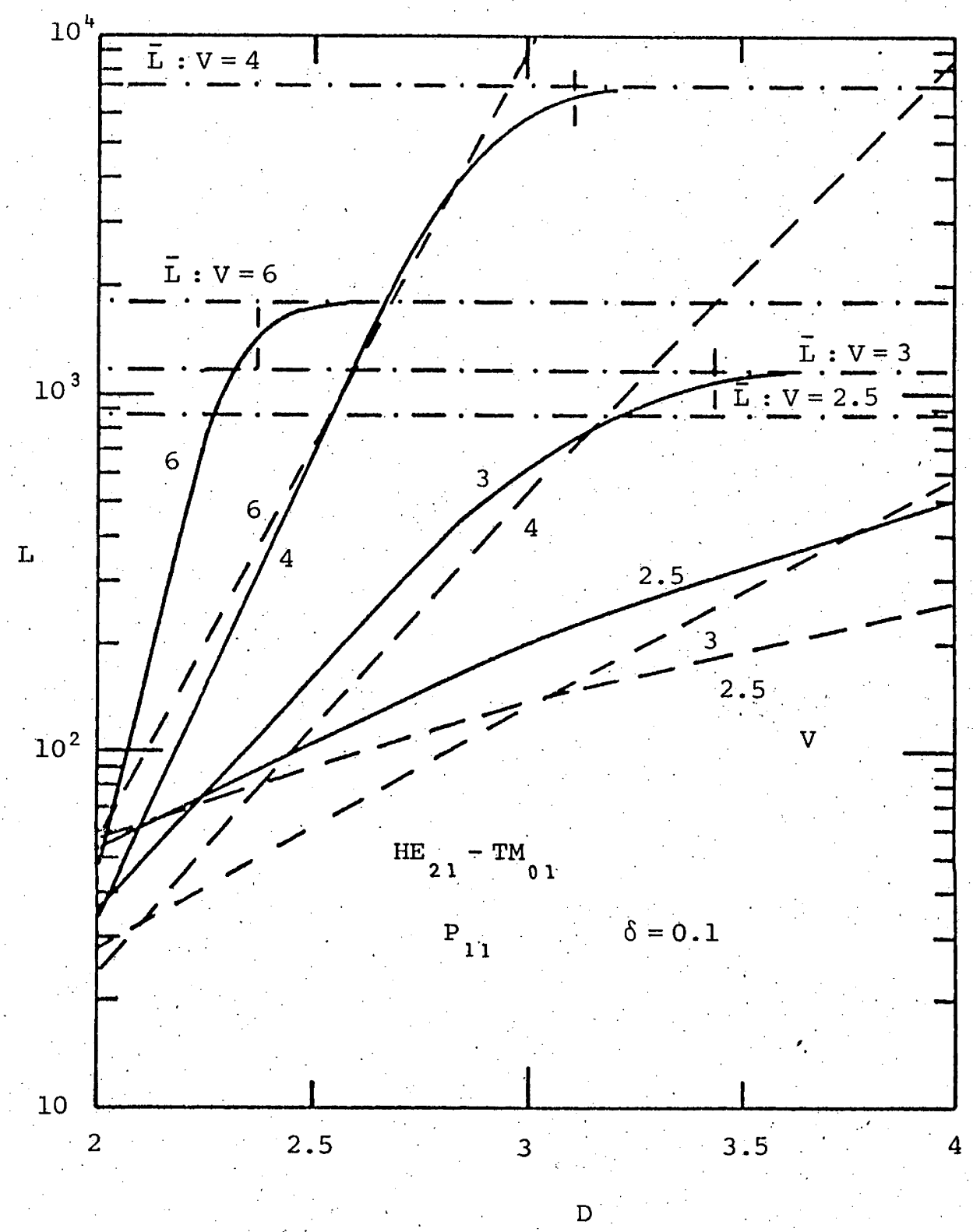

Fig. 3.31. As for Fig. 3.30, but with $\delta=0.1$. 

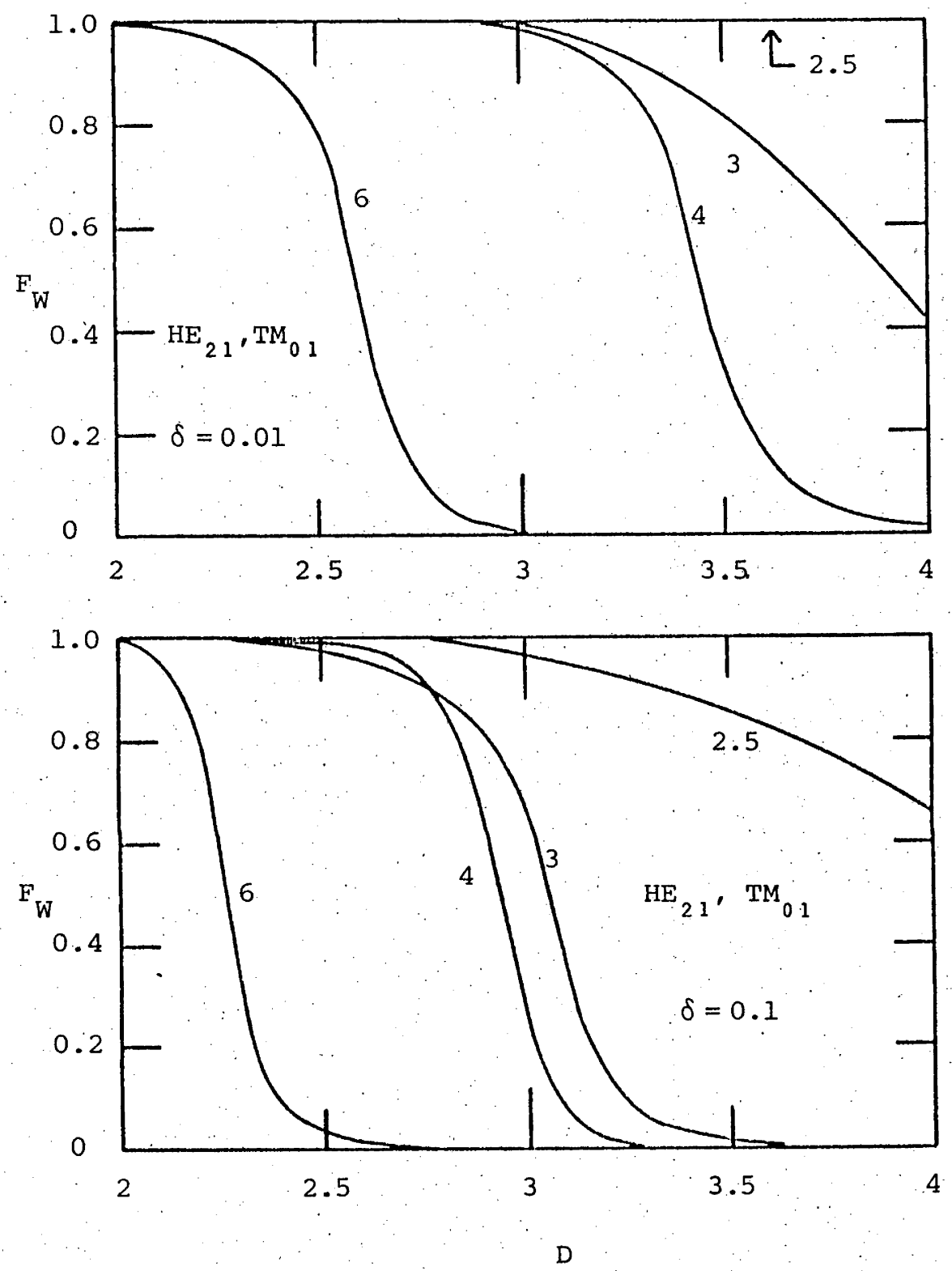

Fig. 3.32. Maximum power transfer $\mathrm{F}_{\mathrm{W}}$ between an $\mathrm{HE}_{2.1}$ and a $\mathrm{TM}_{01}$ mode on one fibre, in the presence of an identical neighbouring fibre at distance $D=d / \rho$ away. 
for which they may be used in such coupling problems is not the length $\overline{\mathrm{L}}$ given by (3.31), but the length $\mathrm{L}_{\mathrm{W}}$ given by (3A.4). In other words, the presence of a neighbouring waveguide speeds up the decomposition of the $\mathrm{P}$ modes. In the limit $\left|\frac{\rho \beta_{d}}{2 \sqrt{\delta K}}\right| \gg>1$, moving the waveguides apart for example, $F_{W} \rightarrow 0$ and $L_{W} \rightarrow \bar{L}$.

Because the $\mathrm{P}_{\ell \mathrm{m}}$ modes are not true modes, it is sometimes of no advantage to use them as a basis, for mode coupling between neighbouring fibres; this is outlined above. In these cases coupling between more than two modes must be considered and it is probably easier to use the true waveguide modes $\mathrm{HE}_{\ell \mathrm{m}^{\prime}} \mathrm{EH}_{\ell \mathrm{m}}$. We give below coupling coefficients for coupling between these modes on neighbouring fibres for the case $\beta_{1}=\beta_{2}$ (see Section 3.5 .3 )

$$
\begin{aligned}
& { }^{H E_{\ell_{1} m_{1}}-H E_{\ell_{2} m_{2}}} \\
& K_{12}=(-1)^{l_{2}-1} \frac{\mathrm{U}_{1} \mathrm{U}_{2}}{\left(\mathrm{~V}_{1} \mathrm{~V}_{2}\right)^{3 / 2}} \\
& \times \frac{\mathrm{k}_{\ell_{1}-\ell_{2}}(\mathrm{WD})}{\left[\mathrm{K}_{\ell_{1}}\left(\mathrm{w}_{1}\right) \mathrm{k}_{\ell_{1}-2}\left(\mathrm{w}_{1}\right) \mathrm{k}_{\ell_{2}}\left(\mathrm{w}_{2}\right) \mathrm{K}_{\ell_{2}-2}\left(\mathrm{w}_{2}\right)\right]^{\frac{1}{2}}} \quad\left\{\begin{array}{l}
\cos \left(\ell_{1}-\ell_{2}\right) \alpha \\
\sin \left(\ell_{1}-\ell_{2}\right) \alpha
\end{array}\right\} \\
& \mathrm{EH}_{\ell_{1} \mathrm{~m}_{1}-\mathrm{EH}_{\ell_{2} \mathrm{~m}_{2}}} \\
& K_{12}=(-1)^{l_{2}+1} \frac{U_{1} U_{2}}{\left(V_{1} V_{2}\right)^{3 / 2}} \\
& \times \frac{\mathrm{K}_{\ell_{1}-\ell_{2}}{ }^{(\mathrm{WD})}}{\left[\mathrm{K}_{\ell_{1}}\left(\mathrm{w}_{1}\right) \mathrm{K}_{\ell_{1}+2}\left(\mathrm{w}_{1}\right) \mathrm{K}_{\ell_{2}}\left(\mathrm{w}_{2}\right) \mathrm{K}_{\ell_{2}+2}\left(\mathrm{w}_{2}\right)\right]^{\frac{1}{2}}} \quad\left\{\begin{array}{l}
\cos \left(\ell_{1}-\ell_{2}\right) \alpha \\
\sin \left(\ell_{1}-\ell_{2}\right) \alpha
\end{array}\right\}
\end{aligned}
$$




$$
\begin{aligned}
& \frac{\mathrm{HE}_{\ell_{1} \mathrm{~m}_{1}}-\mathrm{EH}_{\ell_{2} \mathrm{~m}_{2}}}{\mathrm{~K}_{12}=(-1)^{\ell_{2}+1} \frac{\mathrm{U}_{1} \mathrm{U}_{2}}{\left(\mathrm{~V}_{1} \mathrm{~V}_{2}\right)^{3 / 2}}} \\
& \times \frac{\mathrm{K}_{\ell_{1}}+\ell_{2}(\mathrm{WD})}{\left[\mathrm{K}_{\ell_{1}}\left(\mathrm{~W}_{1}\right) \mathrm{K}_{\ell_{1}-2}{ }^{\left.\left(\mathrm{W}_{1}\right) \mathrm{K}_{\ell_{2}}\left(\mathrm{~W}_{2}\right) \mathrm{K}_{\ell_{2}+2}\left(\mathrm{~W}_{2}\right)\right]^{\frac{i}{2}}} \quad\left\{\begin{array}{c}
\cos \left(\ell_{1}+\ell_{2}\right) \alpha \\
-\sin \left(\ell_{1}+l_{2}\right) \alpha
\end{array}\right\}\right.}
\end{aligned}
$$

where in all cases, the cos $(\sin )$ symmetry arises when the two modes have the same (opposite) symmetry as given by (3.14).

Summary of Approximations and Assumptions

(1) We assume that the fields of a two-waveguide system are superpositions of the fields of the individual waveguides. This is introduced in Section 2.3 .2 and discussed in more detail in section 3.5.3. See Fig. 3.27 and wijngaard (1973).

(2) We assume that the total power is given by the sum of the modal powers: The conditions for power conservation are examined in Section 2.3.2 and in section 3.5.2.

(3). The $\mathrm{P}_{\text {lm }}$ modes, valid only over a limited length of waveguide (except $P_{0 m}$ modes), are used as waveguide modes. This is discussed in Section 3.4 .

(4) The $\mathrm{P}_{\ell \mathrm{m}}$ modes are used to treat coupling between. neighbouring waveguides. The presence of a neighbouring waveguide speeds up the decomposition of the $\mathrm{P}$ mode, as examined above in this Appendix. Again the $\mathrm{P}_{\text {om }}$ modes are an exception. 
REFERENCES

Adler, R.B. (1949), "Properties of guided waves on inhomogeneous cylindrical structures", M.I.T. Technical Report No. 102.

Bracey, M.F., Cullen, A.L., Gillespie, E.F.T. and Staniforth, J.A. (1959), "Surface wave research in Sheffield", I.R.E. Trans. Ant. Prop. AP-7, 5219.

Burke, J.J. (1967), "Switching with fiber-optical waveguides", J. Opt. Soc. Am. 57, 1056.

Clarricoats, P.J.B. (1975), "Theory of optical fibre waveguides a review", in Progress in Optics, E. Wolf ed. (North-Holland Publishing Co., Amsterdam).

Collin, R.E. (1961), Field Theory of Guided Waves (McGraw-Hill, New York).

Enoch, J.M. (1963), "Optical properties of retinal receptors", J. Opt. Soc. Am. 53, 71:

Gloge, D. (1971) "Weakly guiding fibers", Appl. Opt. 10, 2252.

Jones, A.I. (1965), "Coupling of optical fibers and scattering in fibers", J. Opt. Soc. Am. 55, 261.

Kapany, N.S. (1967), Fiber Optics Principles and Apptications (Academic Press, New York).

Kapany, N.S., Burgwald, G.M. and Burke, J.J. (1965), "Light amplification and switching using fiber optics and laser", in Optical and Electro-optical Information Processing, J.T. Tippett et al (eds.). (M.I.T. Press, Cambridge), Ch.17.

Kapany, N.S. and Burke, J.J. (1972), Optical Waveguides (Academic Press, New York).

Kuwahara, H., Hamasaki, J. and Saito, S. (1975), "Power transfer of a parallel optical fiber directional coupler", I.E.E.E. Trans. Microwave Theory Tech. MTT-23, 178.

Marcatili, E.A.J. (1969); "Dielectric rectangular waveguide and directional coupler for integrated optics", B.S.T.J. $\underline{48}, 2071$.

Marcuse, D. (1971), "The coupling of degenerate modes in two parallel dielectric waveguides", B.S.T.J. 50, 1791.

Marcuse, D. (1972), Light Transmission Optics (Van Nostrand Press, New York). 
Marcuse, D. (1973a), "Coupled mode theory of round optical fibers", B.S.T.J. $\underline{52}, 817$.

Marcuse, D. (1974), Theory of Dielectric Optical Waveguides (Academic Press, New York).

Midwinter, J. (1975), "The prism-taper coupler for the excitation of single modes in optical transmission fibres", Optical and Quantum Elect. I., 297.

Snitzer, E. (1961), in Advances in Quantum Electronics, J.R. Singer, ed. (Columbia University Press, New York).

Snitzer, E. (1963), in Optical Processing of Information, D.K. Pollock et al, eds. (Spartan Books, Baltimore).

Snyder, A.W. (1969), "Wave propagation along dielectric structures with application to retinal receptors", Ph.D. Thesis, University of London.

Snyder, A.W. (1969a), "Asymptotic expressions for eigenfunctions and eigenvalues of a dielectric or optical waveguide", I.E.E.E. Trans. Microwave Theory Tech. MTT-17, 1130.

Snyder, A.w. (1969b), "Excitation and scattering of modes on à dielectric or optical fiber", I.E.E.E. Trans. Microwave Theory Tech. MTT-17, 1138.

Snyder, A.W. (1971a) "Approximate eigenvalues for a circular rod of arbitrary relative permittivity", Electron. Letts. 7, 105 .

Snyder, A.W. (1972), "Coupled-mode theory for optical fibers", J. Opt. Soc. Am. 62, 1267.

Snyder, A.W. and Pask, C. (1972), "Light absorption in the bee photoreceptor", J. Opt. Soc. Am. 62, 998.

Stratton, J.A. (194I), Electromagnetic Theory (McGraw-Hill, New York).

Taylor, H.F. and Yariv, A. (1974), "Guided wave optics", PrOC. I.E.E.E. 62, 1044.

Vanclooster, R. and Phariseau, P. (1970), "The coupling of two parallel dielectric fibers I. Basic equations", Physica 47, 485.

Vanclooster, R. and Phariseau, P. (1970a), "The coupling of two parallel dielectric fibers II. Characteristics of the coupling in two fibers", Physica 47, 501.

Vanclooster, R. and Phariseau, P. (1970b), "Diffraction of an electromagnetic wave by a fiber bundle", Physica 50, 308. 
Wijngaard, W. (1971), "Depolarization of plane-polarized light by light-guiding frog rods", J. Opt. Soc. Am. 61, 1187.

Wijngaard, w. (1973), "Guided normal modes of two parallel circular dielectric rods", J. Opt. Soc. Am. 63, 944.

Wijngaard, W. (1974), "Some normal modes of an infinite hexagonal array of identical circular dielectric rods", J. Opt. Soc. Am. 64, 1136. 
CHAPTER 4

POWER TRANSFER BETWEEN OPTICAL FIBRES

WITH VARIABLE COUPLING

\subsection{OUTLINE}

Coupled-mode theory is used to find the power transfer between neighbouring identical fibres when the coupling is dependent on the position along the fibres as in non-parallel or tapered fibres. If the coupling coefficient and the propagation constant are replaced by averaged values, the usual coupled-mode solutions are found to apply (Section 4.3). The theory is applied to non-parallel and tapered fibres, and curves of the averaged coupling coefficient are presented in a length-independent form for $\mathrm{HE}_{11}$ modes on fibres of arbitrary physical parameters (Section 4.4). A discussion of the generalisations of the theory follows (Section 4.5 ).

Important results in this chapter are (4.3)-(4.8), the

equations for power transfer and Figs. 4.2, 4.3, 4.8,4.9 which give the coupling coefficients.

\subsection{INTRODUCTION}

In the previous chapter we have examined the power transfer between parallel uniform fibres in which the coupling coefficient is independent of the coordinate $z$, i.e. the coupling is constant along the length of the fibres. In this chapter we look at a general class of problems in which the coupling is z-dependent, and specifically at 
two examples:

(1) power transfer between identical, non-paxallel, circular optical fibres, and

(2) power transfer between identical, parallel, conical or tapered optical fibres.

The directional coupler, in which power is transferred from one waveguide to another, is used in integrated optics systems (Marcatili, 1969). To avoid unnecessary oscillation of the power between the two waveguides, the coupling region must be restricted so that only one-way power transfer takes place. One method is gradually to separate the waveguides i.e. a system of two non-parallel waveguides. Coupling only takes place over the region in which the waveguides are close together because of the exponential falloff of coupling with separation (Section 3.5.3). Another possible method of restricting power transfer two waveguides with different tapers - is discussed in Section $4: 5$ as a generalisation of our work. The theory presented here is also used to examine coupling between visual photoreceptors (Chapter 7); the retina of many vertebrates consists of parallel, conical photoreceptors (Walls, 1972), and in some insects neighbouring photoreceptors are known. to be tapered and non-parallel (Boschek, 1971).

A feature of this class of problems is that there may be no trapped modes, i.e. modes that propagate unattenuated, of the fibres in isolation. This presents a difficulty, because finding the power transfer between neighbouring fibres using coupled-mode theory requires a knowledge of the isolated-fibre modes. We circumvent this by allowing only small deviations from uniformity, so that the power lost to other modes or to radiation is not significant e.g. a slight taper or bent fibre with a large radius of curvature. The fields of these fibres, 
called quasi-modes, are then approximately the modes of the appropriate uniform circular fibres (Snyder, 1970, 1971b; Gloge, 1972; Marcuse, 1971a).

\subsection{FORMULATION}

Power transfer between modes on neighbouring waveguides is described by the coupled-mode equations $(2.44),(2.45)$. When a $z-$ dependence is present, such as with tapered or non-parallel waveguides, the coupling coefficient $C$ and the propagation constants $\beta$ may be functions of $z$. For two waveguides with identical propagation constants e.g. identical fibres, the coupled-mode equations for each pair of modes that exchange power become

$$
\begin{aligned}
& \frac{d a_{1}(z)}{d z}+i \beta(z) a_{1}(z)=i C(z) a_{2}(z) \\
& \frac{d a_{2}(z)}{d z}+i \beta(z) a_{2}(z)=i C(z) a_{1}(z)
\end{aligned}
$$

We have assumed in deriving (4.1), (4.2) that there is a plane of symmetry between the coordinate systems of the two waveguides, i.e. the z-axes of the fibres are mirror images in this plane. The distances measured along the axes of the waveguides are then equivalent and are written as $z$ in the equations, rather than $z_{1}, z_{2}$. The fibres need not be mirror images, provided that $\beta_{1}=\beta_{2}$. This concept can be generalised (see Section 4.5).

The solutions of $(4.1),(4.2)$ are given by

$$
\begin{aligned}
& a_{1}(z)=e^{-i \bar{\beta} z}\{p \cos (\bar{C} z)+i q \sin (\bar{C} z)\} \\
& a_{2}(z)=e^{-i \bar{B} z}\{q \cos (\bar{C} z)+i p \sin (\bar{C} z)\}
\end{aligned}
$$

where $p, q$ are the initial $(z=0)$ conditions and 


$$
\begin{aligned}
& \bar{B}(z)=\frac{1}{z} \int_{0}^{z} \beta(z) d z \\
& \bar{C}(z)=\frac{1}{z} \int_{0}^{z} C(z) d z
\end{aligned}
$$

When only mode 1 is excited (with unit power) at $z=0$, the power in the modes is given by

$$
\begin{aligned}
& P_{1}(z)=\cos ^{2}(\bar{C} z) \\
& P_{2}(z)=\sin ^{2}(\bar{C} z) .
\end{aligned}
$$

Complete power transfer takes place over a distance $z=\ell_{T}$ where $\ell_{T}\left|\bar{C}\left(\ell_{T}\right)\right|=\frac{\pi}{2}$.

Equations (4.1) $-(4.8)$ are applicable to any system of two waveguides in which the coupling is z-dependent (subject to certain constraints discussed in Sections $4.4,4.5)$. Unfortunately it is not possible to solve (4.1), (4.2) in general, exactly if the propagation constants are not identical; approximate methods of solution must be used (Rowe, 1962 and Section 4.5). If the propagation constants differ greatly everywhere along the waveguides there is little power transfer.

\subsection{COUPLING COEFFICIENTS AND POWER TRANSFER}

\subsubsection{Non-parallel Fibres}

We consider power transfer between $\mathrm{P}_{01}\left(\mathrm{HE}_{11}\right)$ modes on two: straight, identical, circularly cylindrical, uniform fibres inclined at an angle $\phi$ (Fig. 4.1) and assume that the fibre axes lie in a plane; if not coupling between $x-$ and $y$-polarised modes is possible. As the fibres are straight the uniform-fibre modes are exact. If the fibres were bent, we would use the quasi-modes. In fact, the only difference between using the theory for straight or bent fibres is in the variation of the separation with distance along the fibres. The theory may also be easily 


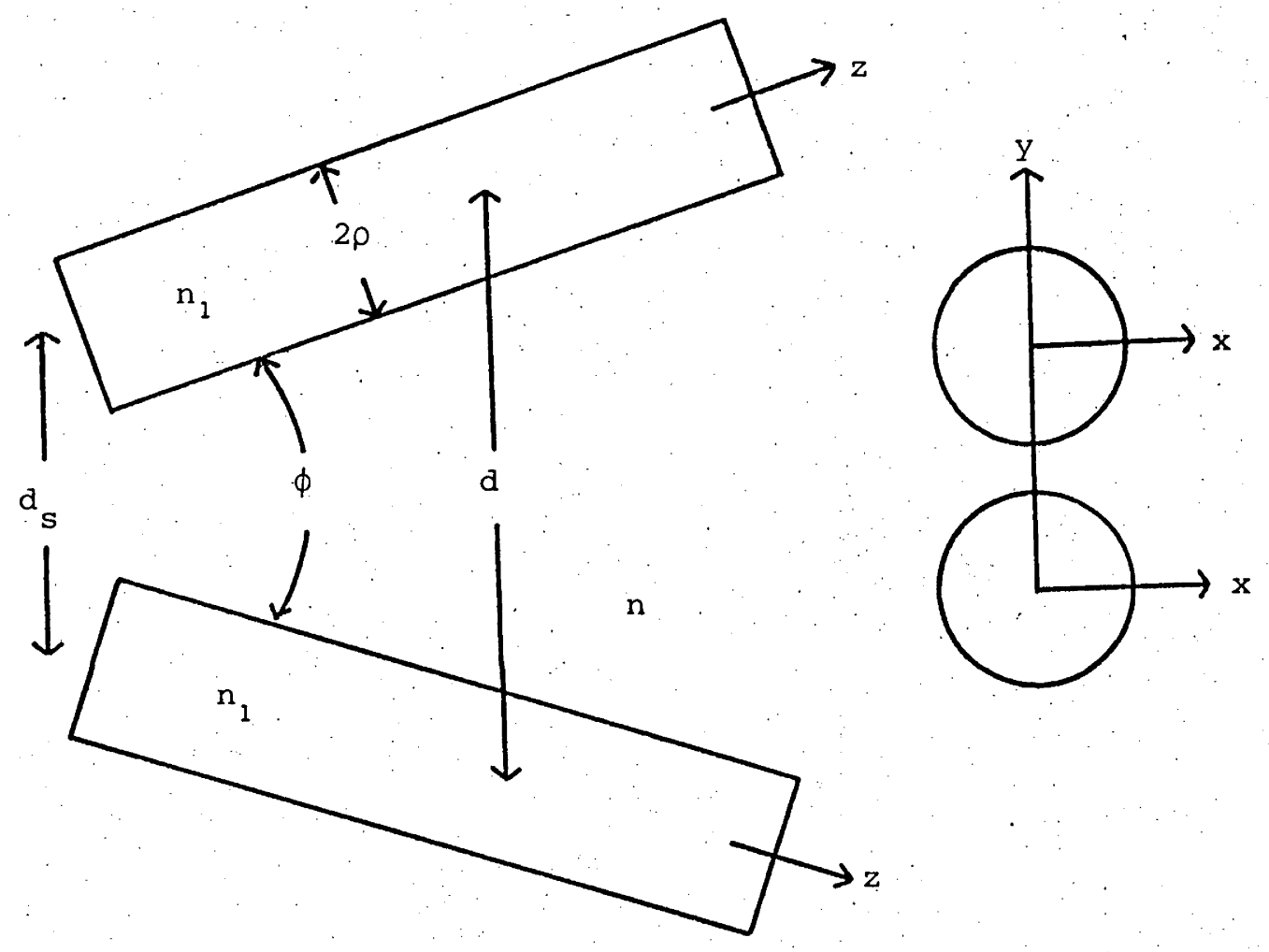

Fig. 4.1. Two identical non-parallel fibres. 
extended to higher-order modes using the P modes of Chapter 3 .

For uniform fibres $\bar{\beta}$ is constant and the coupling constant at a separation $d(z)$ of the fibre axes is given by (3.58) as

$$
K(z)=-\frac{\rho C(z)}{\sqrt{\delta}}=\frac{U^{2}}{v^{3}} \frac{K_{0}(W D)}{K_{1}^{2}(W)}
$$

where $D=d(z) / \rho$. $K$ for the $y$-polarised modes must be multiplied by $\cos \phi$

By changing variables from $z$ to $D=\frac{d(z)}{\rho}=\frac{d s}{\rho}+\frac{2 z}{\rho} \tan \frac{\phi}{2}$, we can write $(4.6)$ in a length-independent form

$$
\bar{K}=-\frac{\rho \bar{C}}{\sqrt{\delta}}=\frac{1}{D-D_{S}} \int_{D_{S}}^{D} K(D) d D
$$

where $D_{S}=d_{S} / \rho=D(z=0)$. The form (4.10) allows the presentation on one graph of curves of $\bar{k}$ for fibres of arbitrary length with a given initial separation. Substitution of (4.9) into (4.10) gives

$$
\bar{K}(D)=\frac{U^{2}}{V^{3}} \frac{1}{K_{1}^{2}(W)} \frac{1}{D-D_{S}} \int_{D_{S}}^{D} K_{0}(W D) d D
$$

The value of $D$ indicates position along the fibres because $D=D(z)$. Families of curves of $\bar{k}$ vs. $V$ for $P_{02}$ modes are presented for $D_{S}=2$ in Fig. 4.2 and for $D_{S}=4$ in Fig. 4.3. Figure 4.4 shows the effect of varying the initial separation $D_{S}$. Curves for parallel fibres are included on all figures for comparison. For touching fibres, the initial separation $D_{S}=\sqrt{2(1+\cos \phi)} \approx 2$ for $\operatorname{small} \phi$ :

The length for total power transfer $l_{T}$ is given by

$$
L_{T}=\frac{\ell_{T}}{\rho}=\frac{\pi}{2 \sqrt{\delta} \bar{K}}
$$

The parameters to be specified are $V, \delta, D_{S}$ and $\frac{D\left(l_{T}\right)}{D_{S}}$. This determines the following situation: given two fibres of length $l$ with normalised. 


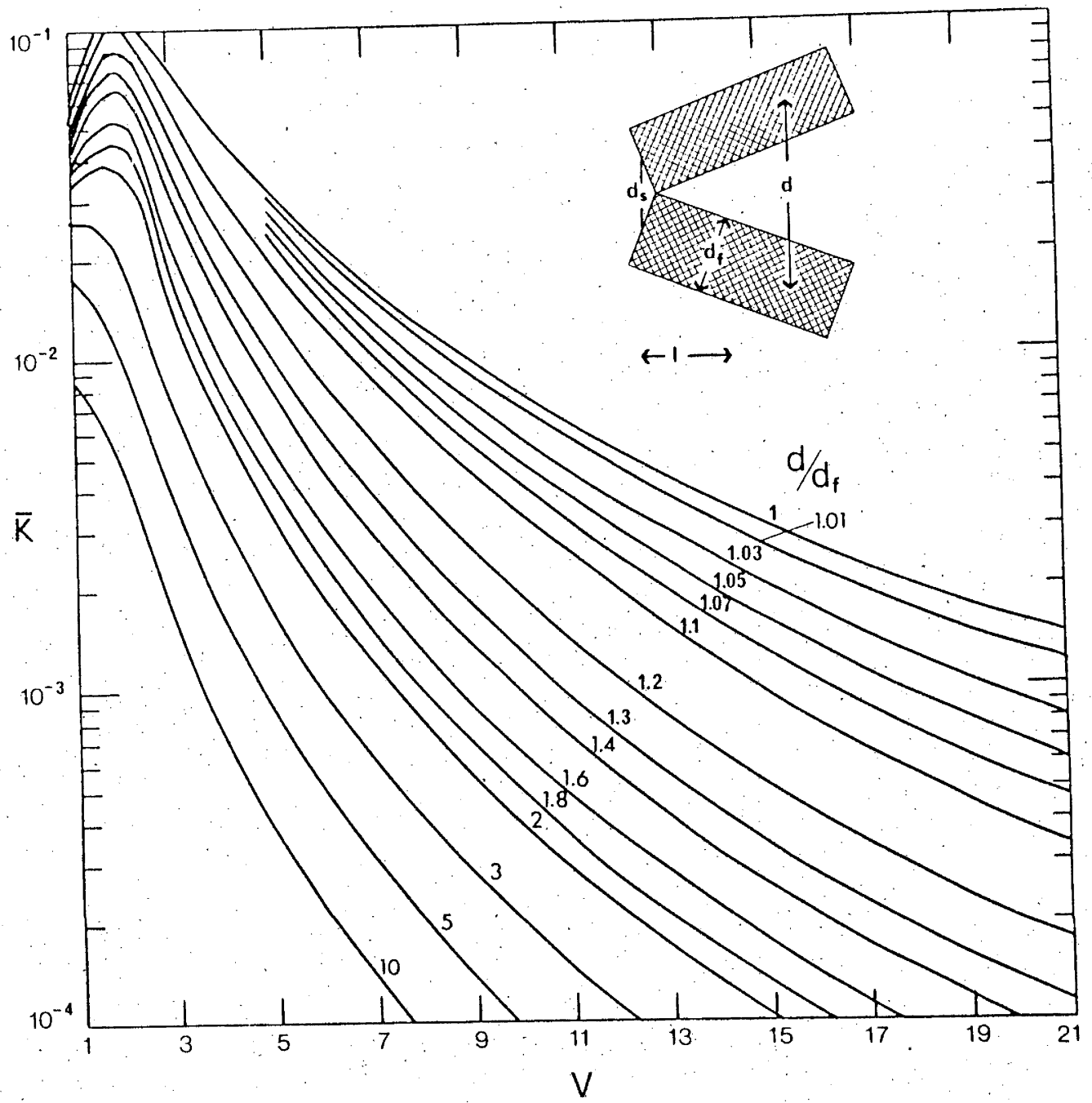

Fig. 4.2. Averaged coupling constant $\bar{K}$ vs. $V$ for $P_{0}$ modes on two identical non-paraliel fibres with initial separation equal to twice the fibre radius $\left(D_{s}=d_{s} / p=2\right) \ldots$ For small inclination $\phi$, the fibres are approximately touching. Complete power transfer takes place over a distance $z=\ell$, where $\ell / \rho=\pi / 2 \bar{\kappa} \sqrt{\delta}$. 


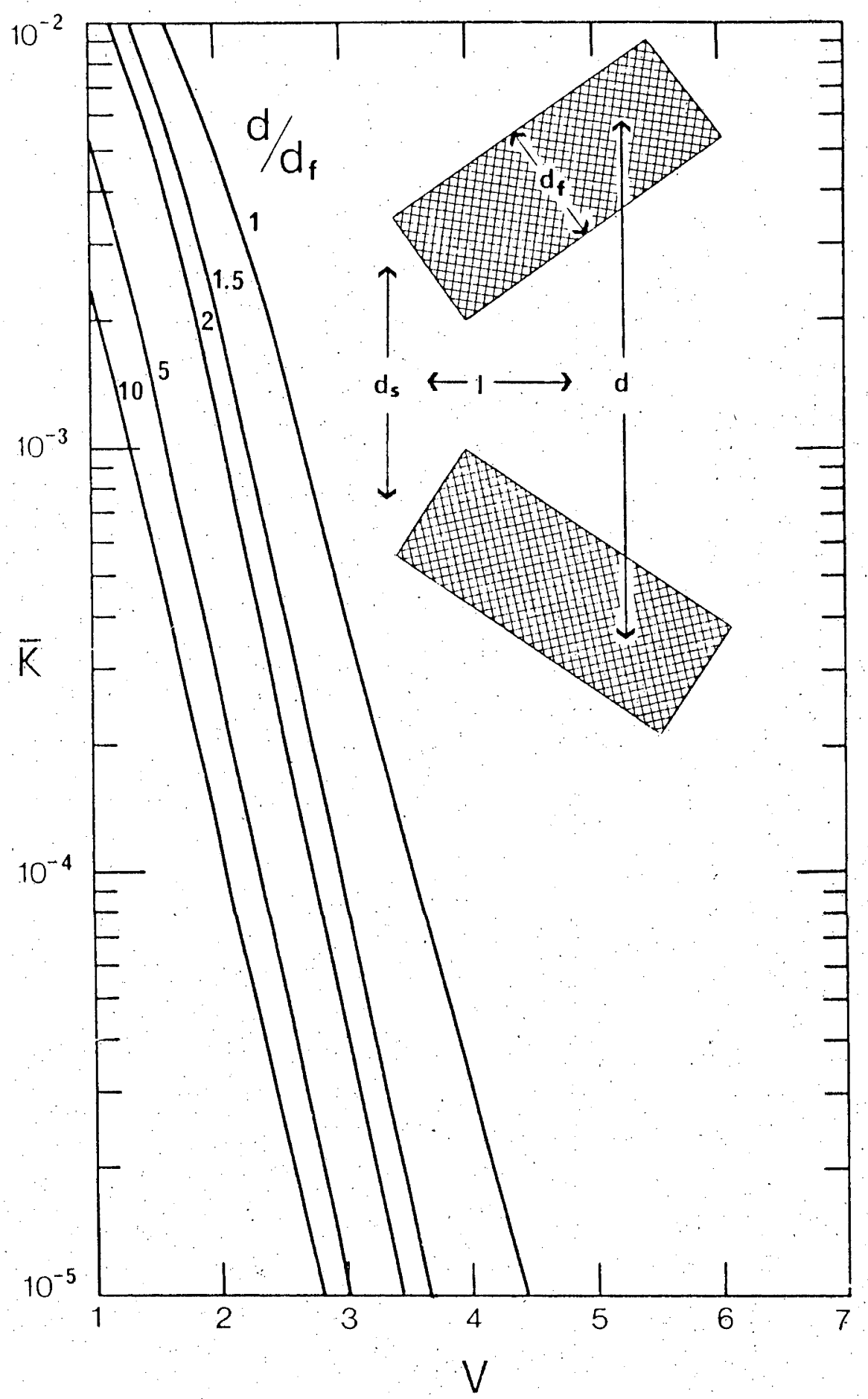

Fig. 4.3. As for Fig: 4.2, but with $D_{S}=4$. 


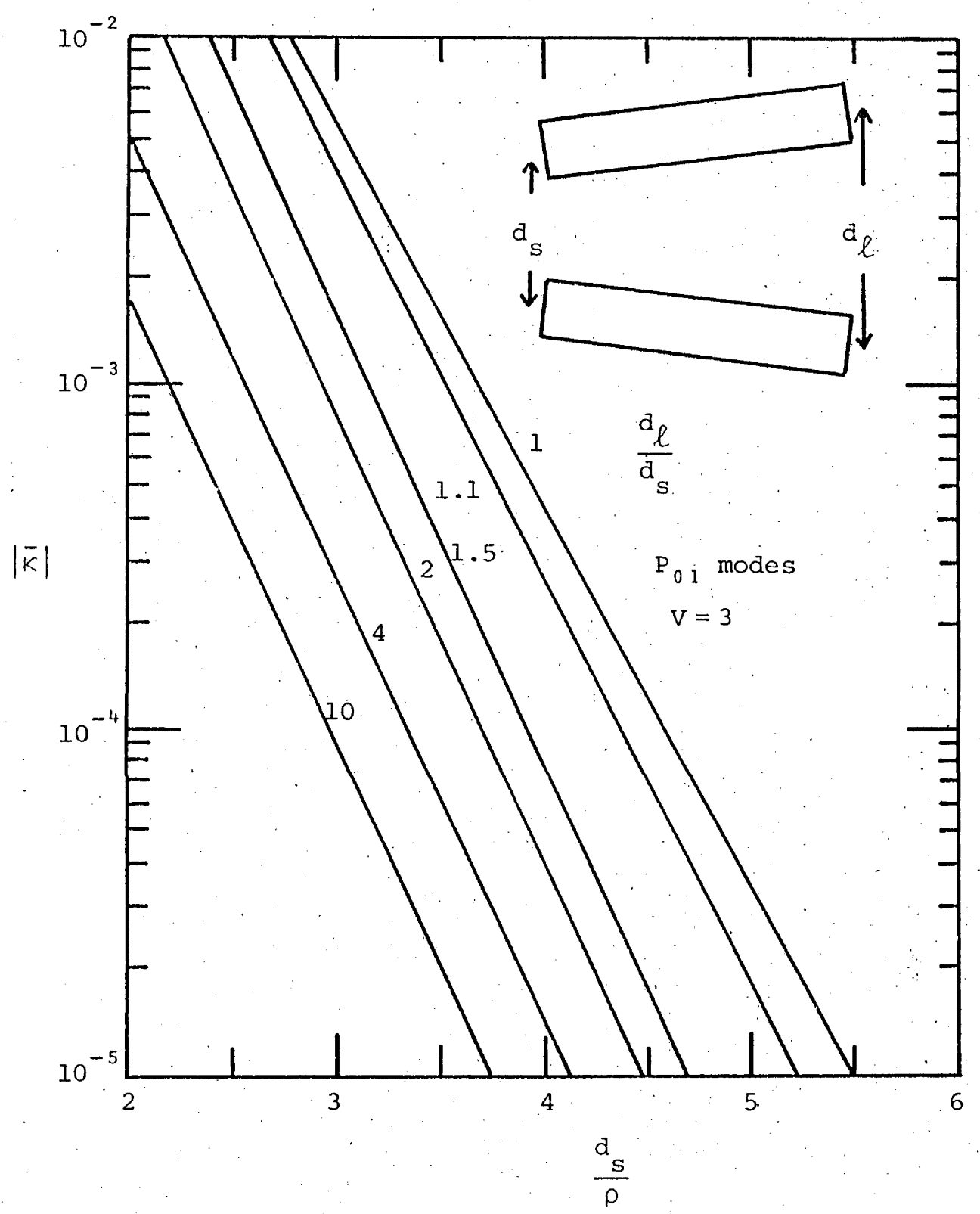

Fig. 4.4. Averaged coupling constant $\bar{K}$ as a function of $D_{S}=d_{S} / \rho$ for $P_{01}$ modes on two nonparallel identical fibres. $V=3$. 
initial separation $D_{S}$, how far apart must the ends be to give total power transfer - i.e. we vary $\frac{D}{D_{S}}$ until the right-hand side of (4.10) gives the required length $\ell$. Figure 4.5 shows the length of fibres required for total power transfer given $\mathrm{D}_{\mathrm{S}}=2, \mathrm{~V}=3$ and $\delta$ as a function of $\frac{D\left(l_{T}\right)}{D_{S}}$.

To find the length for total power transfer between two fibres inclined at an angle $\phi$ given $\mathrm{D}_{\mathbf{S}^{\prime}}$ we must solve the transcendental equation $\ell \bar{C}(\ell)=\frac{\ell \sqrt{\delta} \bar{K}(\ell)}{\rho}=\frac{\pi}{2}$.

In Fig. 4.6 we show the power distribution as a function of $\mathrm{z}$ in neighbouring non-parallel fibres with $\mathrm{V}=3, \delta=0.1, \mathrm{D}_{\mathrm{S}}=2$, where initially all the power is on one fibre. Curves for parallel fibres are given for comparison. This figure demonstrates the decrease in the rate of power transfer with increasing separation i.e. increasing $\frac{D}{D_{S}}$. In using the coupled-mode theory of Chapter 2 for nonparallel fibres, we need to look closely at some of the assumptions implicit in the formalism. The coupling coefficient is found from an integration over a cross-sectional plane at right angles to the fibre axes. This arises from the mode orthogonality condition $(2.10)$ and sets up a $1: 1$ correspondence between positions on the two fibres. When the fibre axes are not parallel we must modify this 1:1 correspondence and assume that the field at a position $\mathrm{z}$ on one fibre is perturbed by the field at the corresponding position $z$ on the other fibre. This may be generalised when there is no plane of symmetry between the fibres (Matsuhara and Watanabe, 1975). From the geometry of the system we expect this to be a good approximation when the angle of inclination $\phi$ of the fibres is small, but to break down when $\phi$ is large and the separation greater than several fibre radii. However if these conditions 


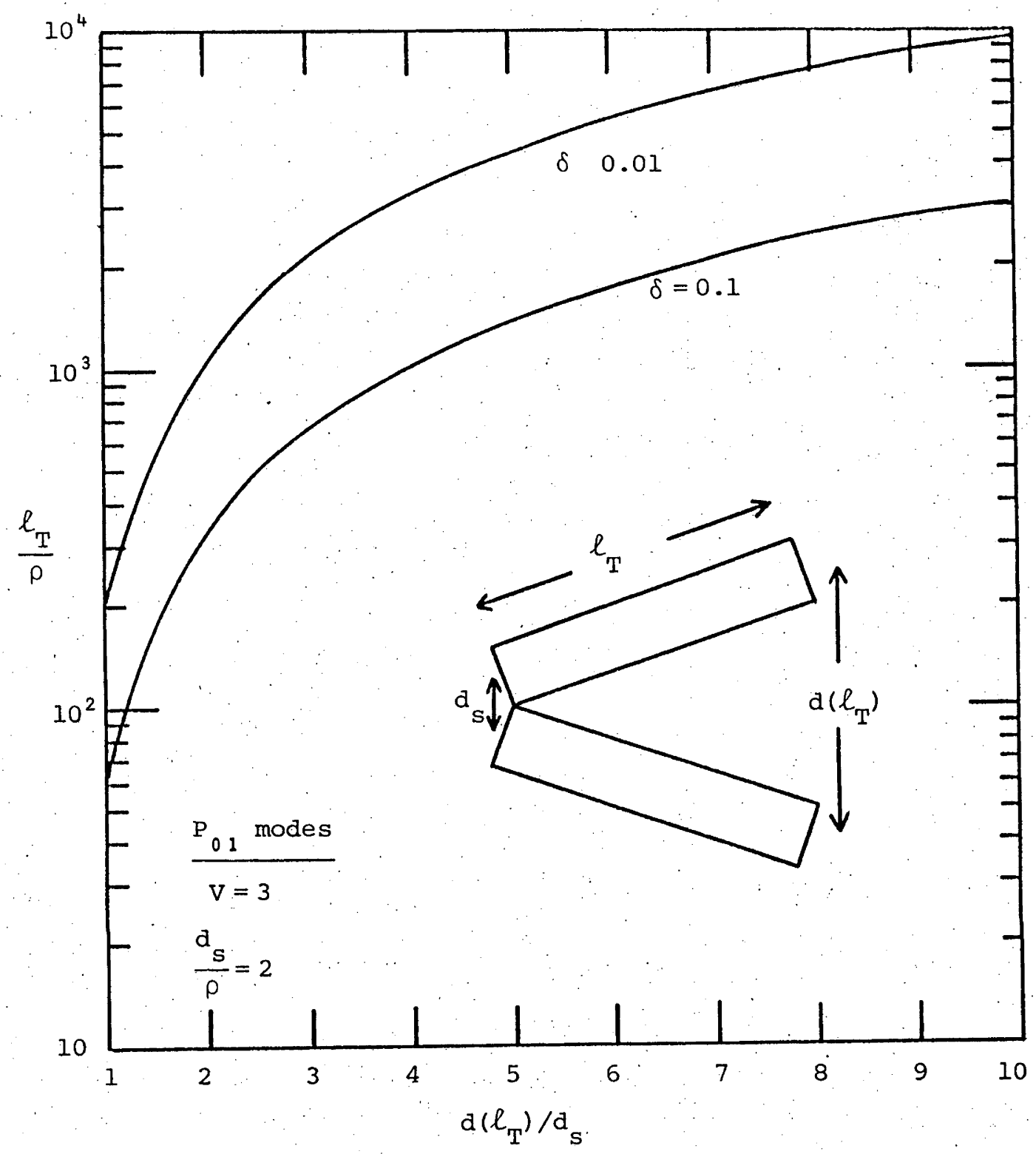

Fig. 4.5. Length $\ell_{T}$ for total power transfer between two identical non-paraliel fibres of radius $\rho$. $D_{S}=\frac{d_{S}}{\rho}=2$ and $\frac{D}{D_{S}}=\frac{d}{d_{s}}$. 


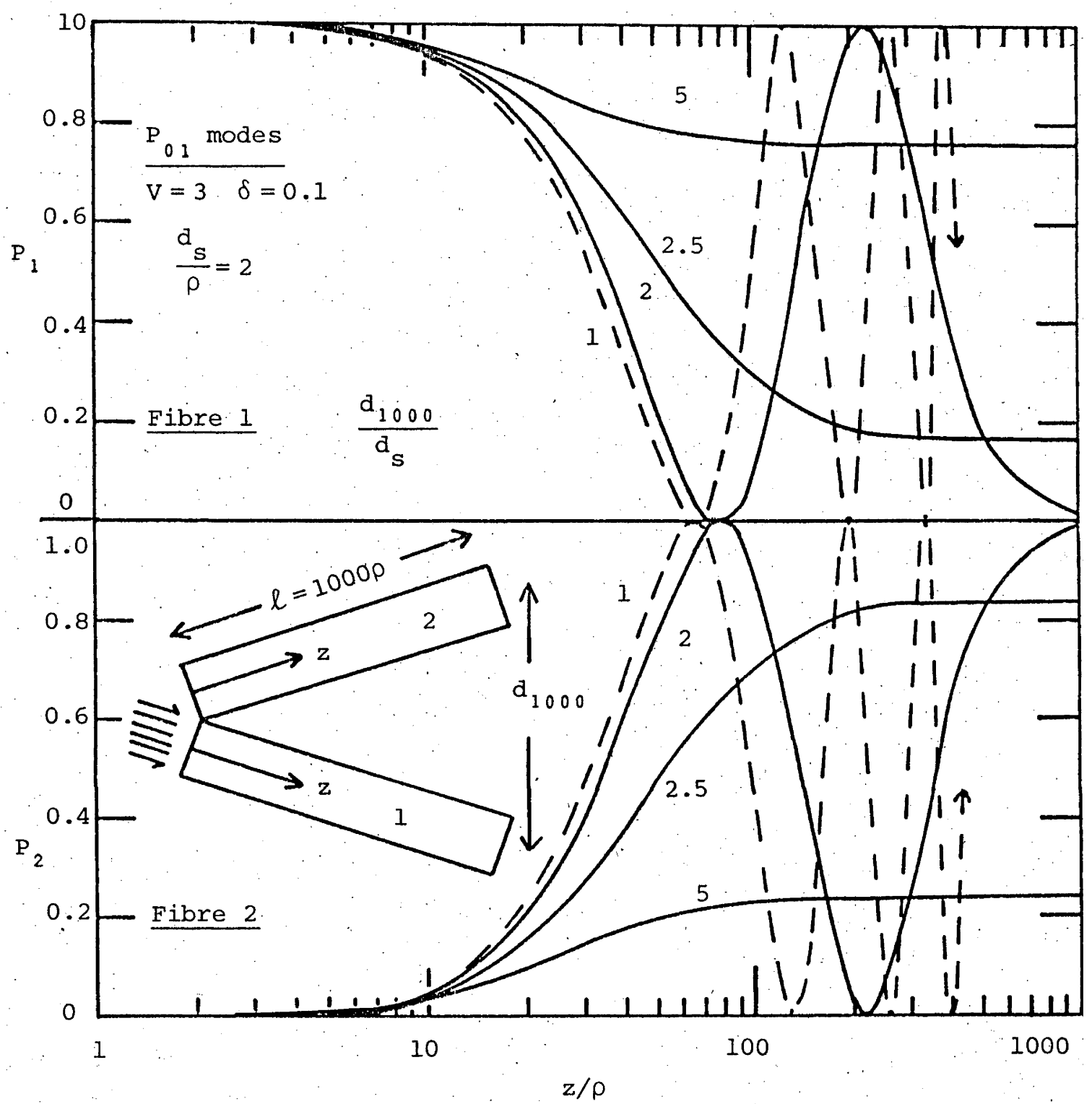

Fig. 4.6. Power in two coupled identical, non-parallel fibres as a function of distance along the fibres. Fibre 1 is excited with unity initial power. The dashed line is for parallel fibres $\left(\frac{d_{1000}}{d_{S}}=1\right)$ and is omitted for large $z / \rho$ because it oscillates too rapidly. 
are present little coupling generally takes place anyway. Provided that the fibre axes are approximately parallel when the fibres are close together, the theory should give a good approximation to the actual power transfer.

\subsubsection{Tapered Fibres (linear tapex)}

The concept of modes for a uniform cylindrical fibre may be retained for tapered fibres, provided that the taper is slight at every position $z$ along the fibre and $\delta \ll 1$. We assume that the "modes" of a conical fibre are what Snyder $(1970,1971 b)$ calls local modes. These local modes have the property that at any position $z$ along the fibre, they are the modes of a uniform fibre of radius $\rho(z)$. Clearly these modes do not exactly satisfy the conical boundary conditions and therefore couple to each other. However. Snyder $(1970,1971 b)$ has shown that the local $\mathrm{HE}_{11}$ mode propagates along the conical fibre with very. little loss to other modes or to radiation: Power transfer between local $\mathrm{HE}_{11}$ modes on neighbouring identical tapered fibres is therefore described by $(4.1)-(4.8)$. In general, provided the rate of change of radius is small compared with the radius, we can probably neglect coupling between local $P$ modes on short fibres as long as the mode does not pass through cutoff.

For tapered fibres $\rho, V, U, W$ are all functions of $z_{i}$ for a linear taper $\rho$ and $\mathrm{V}$ are linear functions of $\mathrm{z}$, whereas $\mathrm{U}$ and $\mathrm{W}$ must be found from the eigenvalue equation (3.23), and (3.22). The coupling coefficient is given by

$$
C(z)=-\frac{\sqrt{\delta} U^{2}(z)}{\rho(z) V^{3}(z)} \frac{K_{0}(W(z) D(z))}{K_{1}^{2}(W(z))}
$$

In order to define a length-independent coupling constant, we change variables from $z$ to $t=\rho_{i} / \rho=v_{i} / v$ where $\rho_{i}, v_{i}$ are the values at $z=0$ (Fig. 4.7). Then (4.6) becomes 


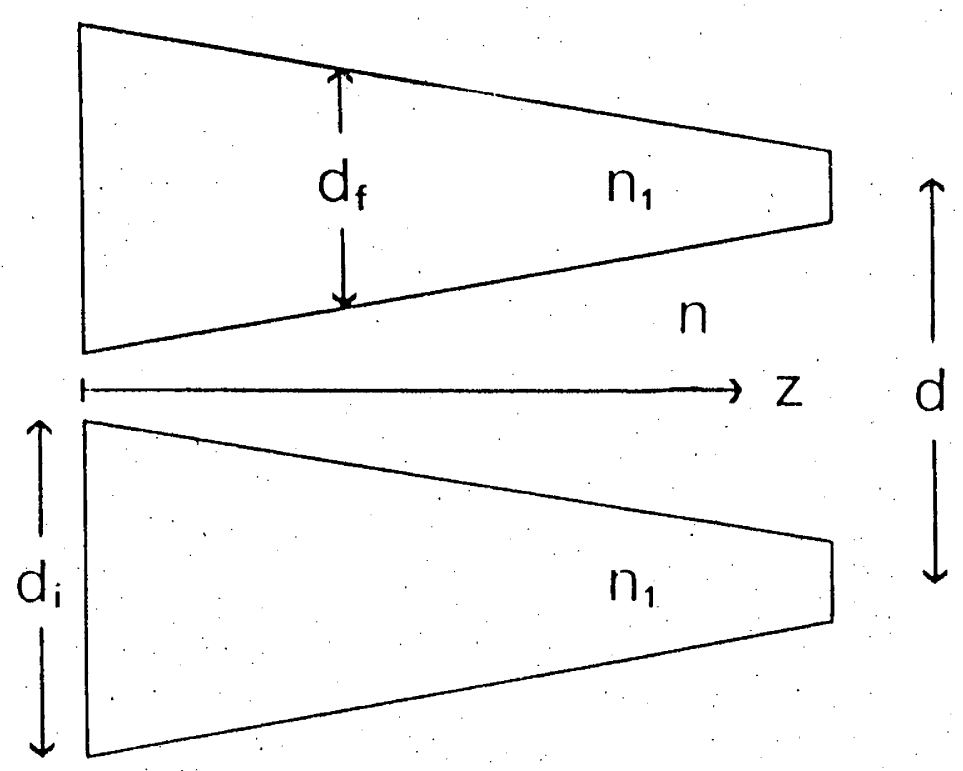

Fig. 4.7. Tapered fibres. $d_{f}=2 \rho_{i} d_{i}=2 p_{i}$.

$$
\bar{C}(R)=\frac{R}{R-1} \int_{1}^{R} d t \frac{C(t)}{t^{2}}
$$

where $R=\rho_{i} / \rho_{0}=v_{i} / V_{0}, V_{0}, \rho_{0}$ are the values of $v, \rho$ at the required length z. Using (4.13),

$$
\bar{K}(R)=-\frac{\rho_{i} \bar{C}(R)}{\sqrt{\delta}}=\frac{1}{V_{i}^{3}}\left(\frac{R}{R-1}\right) \int_{1}^{R} \frac{t^{2} d t U^{2} K_{0}\left(W D_{i} t\right)}{K_{1}^{2}(W)}
$$

where $U=U(V)=U\left(V_{i} / t\right), w^{2}=v^{2}-U^{2}, D_{i}=d / \rho_{i}$. The variable $R$ indicates position along the fibres because $R=R(z)$.

We present families of curves of $\bar{k}$ vs. $v_{i}$ for touching fibres $\left(D_{i}=2\right)$ in Fig. 4.8, and for $D_{i}=4$ in Fig. 4.9. Total power transfer takes place over a length $\ell_{T}$ where $\frac{l_{T}}{\rho_{i}}=\frac{\pi}{2 \sqrt{\delta} \bar{K}}$. The discussion on power transfer in Section 4.4 .1 is again relevant here.

Figure 4.10 shows the power distribution as a function of $z$ for two tapered fibres where initially all the power is in one 


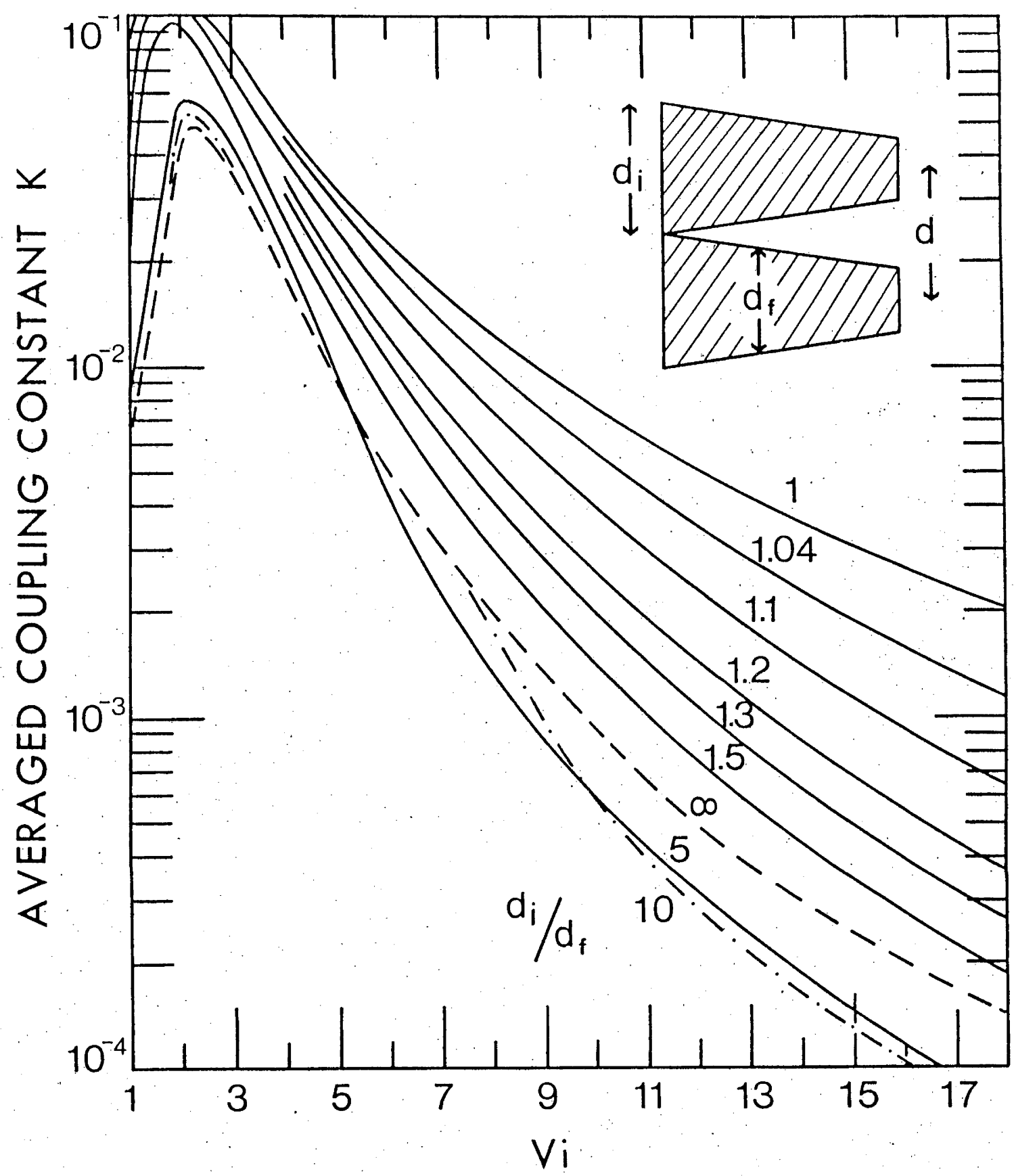

Fig. 4.8. Averaged coupling constant $\bar{k}$ vs. $v_{i}$, the initial $(z=0)$ value of $V$, for $P_{01}$ modes on two identical tapered fibres. The separation is equal to twice the initial radius $\left(D_{i}=d / \rho_{i}=2\right.$-touching fibres). $v_{i} / V=\rho_{i} / \rho=d_{i} / d_{f}$, where $\rho(z)$ is the fibre radius. Complete power transfer takes place over a distance $z=\ell$, where $\ell / \rho_{i} \doteq \pi / 2 \bar{K} \sqrt{\delta}$. 


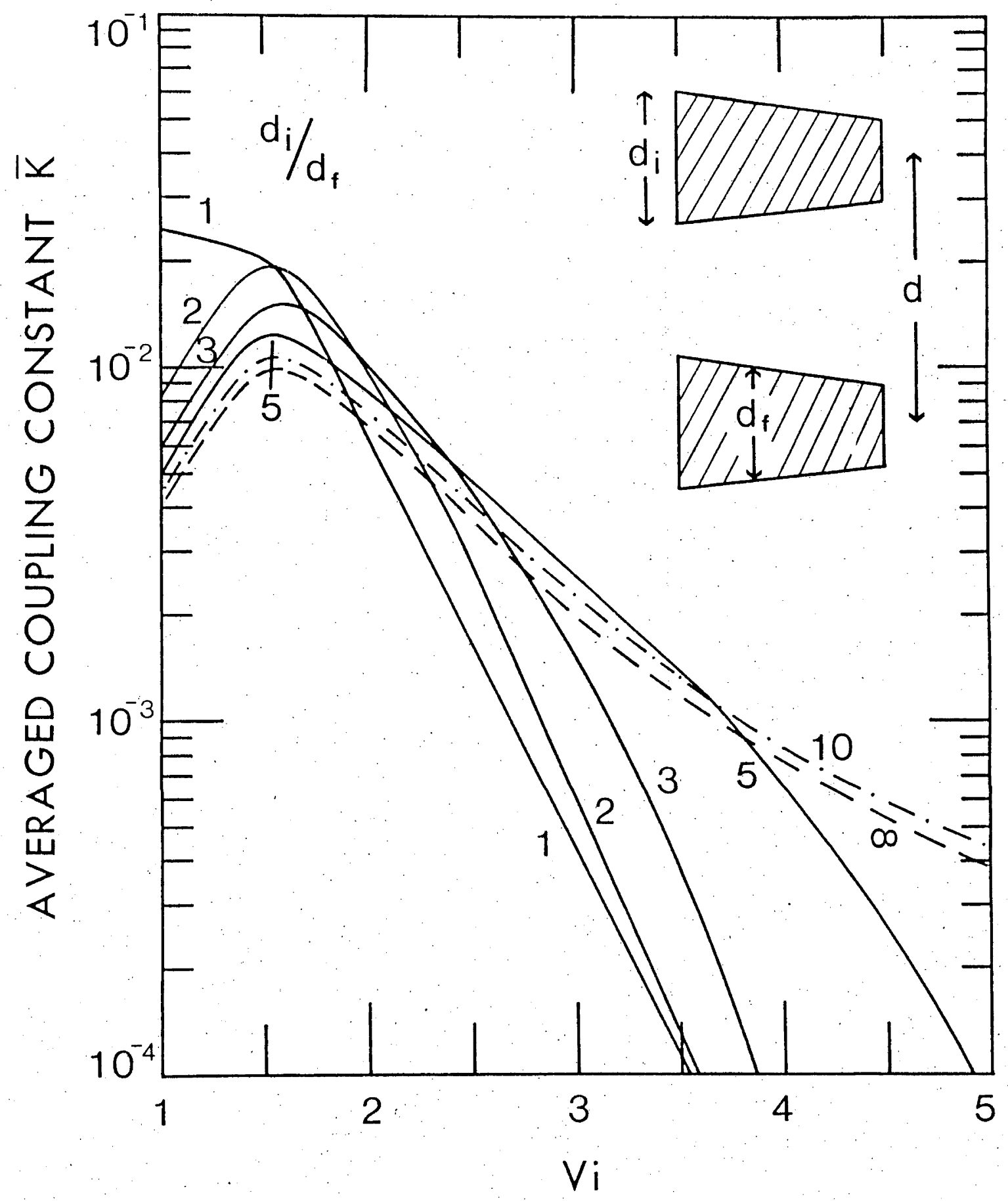

Fig. 4.9. As for Fig. 4.8, but with $D_{i}=4$. $d_{i} / d_{f}=\rho_{i} / \rho=v_{i} / v$. 


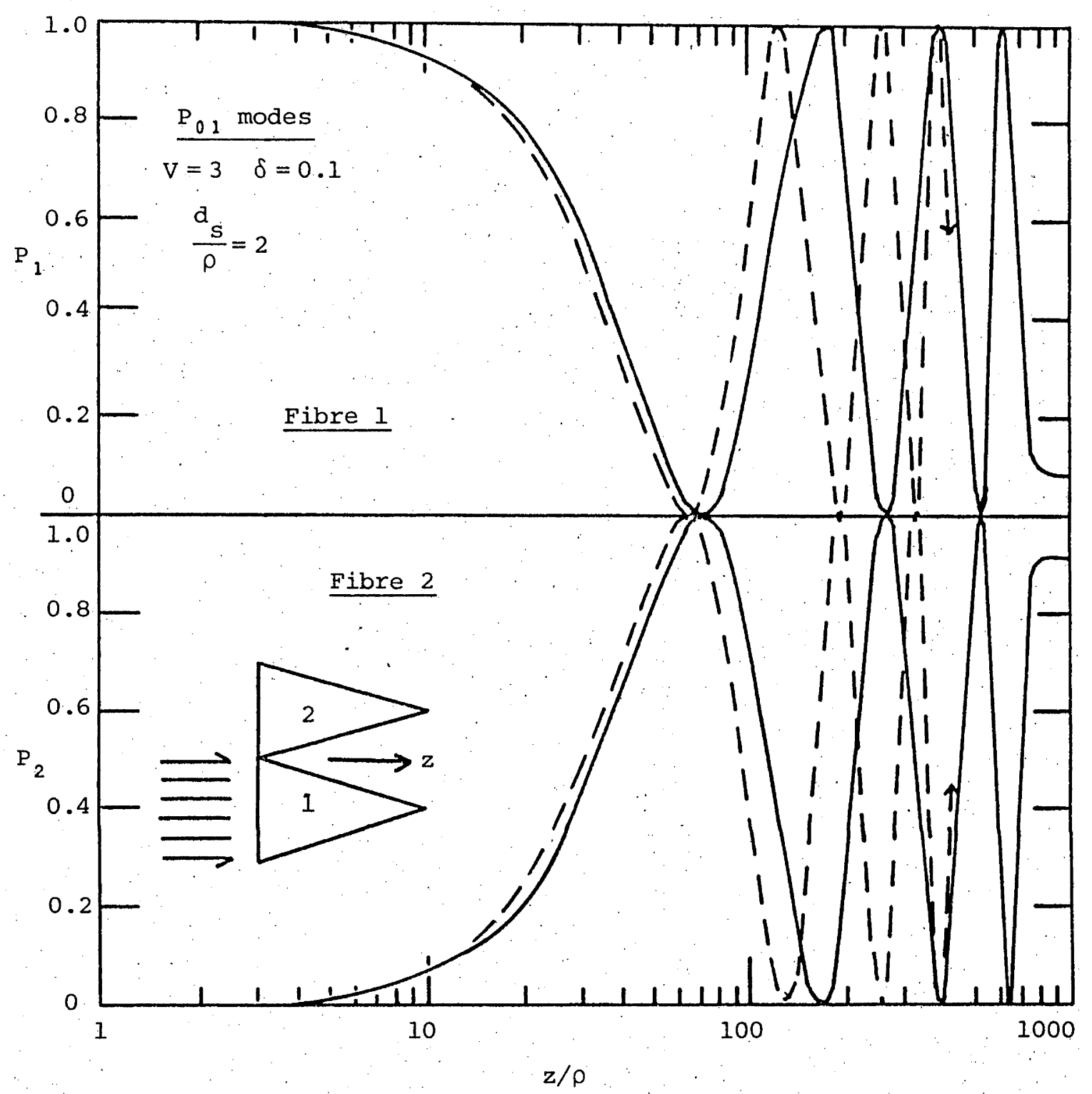

Fig. 4.10, Power in two coupled, identical, touching, tapered fibres as a function of distance along the fibres. Fibre 1 is excited with unity initial power. The case shown is $R=\infty$. (tapering to a point); for $l<R<\infty$, power transfer takes place faster than for $R=\infty$. The dashed line is for untapered fibres $(R=1)$, and, as in Fig. 4.6, is omitted for large $z / \rho$ to avoid confusion with the other curve. 
fibre. The case shown is for fibres tapering to a point with $v_{i}=3$; all other values of $d_{i} / d_{f}$ give greater coupling. The variation of the rate of power transfer with distance $z$ along tapered fibres depends on the diameter of the fibres through two parameters:

(1) the normalised separation $d / \rho$ of the fibres (Fig. 4.7);

(2) the fraction of the power in the mode that propagates inside the fibre (a function of $v$ ).

In the example, as $z$ increases, the fibre radius decreases so that

(1) the normalised separation increases, decreasing the rate of power transfer;

(2) $\mathrm{V}$ decreases so that more of the modal power propagates along but outside the fibre (Snyder, 1969a), increasing the interaction of the fields of the fibre modes, and therefore increasing the rate of power transfer.

The effect of this $V$ variation in compensating for the increased normalised separation can be seen by comparing Fig. 4.10 with the corresponding Fig. 4.6 for non-parallel fibres in which there is only a variation in separation. Mathematically, the dominant term in the coupling constant goes like $\mathrm{e}^{-\mathrm{Vd} / \rho}$ asymptotically; for linearly tapered fibres this is constant, whereas for non-parallel fibres it falls off exponentially with $d$.

For a fixed separation the greatest coupling between $\mathrm{P}_{01}$ modes occurs when $\mathrm{V} \sim 1$, and power transfer is therefore enhanced in the region of each curve of Figs. 4.8, 4.9 where this condition is satisfied. Compare the curves $d_{i} / d_{f}=5,10$ in the region $5<v_{i}<10$ of Fig. 4.8. For this reason $\bar{k}$ for $d_{i} / d_{f}=\infty$ (tapering to a point) is larger than $\bar{k}$ for smaller values of $d_{i} / d_{f}$ if $v_{i}$ is large enough. 


\subsection{DISCUSSION}

An essential feature of the theory presented in this chapter is that the quasi-modes of the individual fibres retain the characteristics of modes of individual fibres. Given this, the theory allows an arbitrary variation in the separation $d(z)$ between the fibre axes, provided that the system remains symmetrical (and subject to the considerations of Section 4.4.1), and a variation in the fibre parameters with $z$, provided that the resulting local modes remain quasi-modes. The method can be extended to a symmetrical linear array of three fibres (see Snyder, 1972) but not to more than three because the effective propagation constants are no longer identical (Section 3.6).

From both a mathematical and a practical point of view we would like to extend the theory to allow for fibres with different propagation constants, varying arbitrarily with $z$. In practice it is difficult to achieve the exact tolerances in the manufacture of directional couplers needed to reproduce theoretical results (Wilson and Teh, 1973, 1975). Unfortunately a general solution to (4.1), (4.2) when $\beta_{1}, \beta_{2}$ are different and different functions of $z$ is not possible, but an analytic solution in terms of known functions has been found for one particular case. This is where there is an arbitrary linear variation of $\beta_{1}, \beta_{2}$ with $z$ and constant coupling coefficients (Jones, 1965). The solution is in terms of parabolic cylinder functions and shows that significant power transfer occurs only when the propagation constants are approximately equal as we would predict from Chapter 2 . The idea of coupled tapered waveguides has been used by Wilson and Teh (1973, 1975), and subsequently by Smith (1975) and Arnaud (1975), to design a "crossedbeta" coupler in which $\beta_{1}, \beta_{2}$ vary in such a way that they become equal 
and crossover at some point within the tapered region of the coupler. Power transfer occurs only within a limited region about the crossover point and because the crossover can be anywhere within the tapered region, some of the manufacturing tolerance is eased. On the basis of the results of section $4: 4.2$ the assumption of a constant coupling coefficient, though not exact, is probably reasonable. Numerical calculations would be necessary to confirm this.

A generalisation of coupling between non-parallel waveguides. is provided by Matsuhara and Watanabe (1975) : who solve (4.1), (4.2) numerically for waveguides with different curvatures and propagation constants. They too assume a $1: 1$ correspondence between positions on the waveguides and calculate the coupling coefficients using the assumptions outlined in Section 4.4.1. Consequently for example, their theory may not be valid for coupling between the extremities of their semicircular-straight waveguide system (their Fig. 3) and in fact is probably only accurate for a small region about the point of closest approach of the waveguides where they are almost parallel. However according to their results it is only in this region where significant power transfer occurs.

$$
\text { In summary, it is possible to generalise our theory by }
$$

finding specific cases in which the coupled-mode equations (4.1), (4.2) can be solved, or by solving them numerically. In all cases this leads to solutions that are more complicated than (4.3), (4.4). Our solutions can be used to give an upper bound on the amount of power transfer in most cases. 
REFERENCES

Arnaud, J.A. (1975), "Transverse coupling in fiber optics part IV: Crosstalk", B.S.T.J. 54, 1431.

Boschek, C.B. (1971), "On the fine structure of the peripheral retina and lamina ganglionaris of the fly. Musca domestica", Z. Zeliforsch. $118,369$.

Gloge, D. (1972), "Bending loss in multimode fibers with graded and ungraded core index", Appl. Opt. 11, 2506.

Jones, A.L. (1965), "Coupling of optical fibers and scattering in fibers", J. Opt. Soc. Am. 55, 261.

Marcatili, E.A.J. (1969), "Dielectric rectangular waveguide and directional coupler for integrated optics", B.S.T.J. 48, 2071.

Marcuse, D. (197la), "Bending losses of the asymmetric slab waveguide", B.S.T.J. 50, 2551 .

Matsuhara, M. and Watanabe, A. (1975), "Coupling of curved transmission lines and application to optical directional couplers",

J. Opt. Soc. Am. 65, 163.

Rowe, H.E. (1962), "Approximate solution for the coupled line equations", B.S.T.J. 41,1011 .

Smith, R.B. (1975), "Coupling efficiency of the tapered coupler", Electron. Lett. 11, 204 .

Snyder, A.W. (1969a), "Asymptotic expressions for eigenfunctions and eigenvalues of a dielectric or optical waveguide", I.E.E.E. Trans. Microwave Theory Tech. MTT-17, 1130.

Snyder, A.W. (i970), "Coupling of modes on a tapered dielectric cylinder", I.E.E.E. Trans. Microwave Theory Tech. MTT-18, 383.

Snyder, A.W. (1971b), "Mode propagation in a nonuniform cylindrical medium", I.E.E.E. Trans. Microwave Theory Tech. MTT-19, 402.

Snyder, A.W. (1972), "Coupled-mode theory for optical fibers", J. Opt. Soc. Am. 62, 1267.

Walls, G. (1972), The Vertebrate Eye (Cranbrook Press, Michigan).

Wilson, M.G.F. and Teh, G.A. (1973), "Improved tolerance in optical directional couplers", Electron. Lett. $\underline{9}, 453$.

Wilson, M.G.F. and Teh, G.A. (1975), "Tapered optical directional coupler", I.E.E.E. Microwave Theory Tech. MTT-23, 85. 


\section{CHAPTER 5}

\section{CROSSTALK BETWEEN MULTIMODE OPTICAL FIBRES}

\subsection{OUTLINE}

Using the mode theory of Chapter 3 we calculate the crosstalk between multimode optical fibres, or light pipes, when one fibre is illuminated by a focussed collimated beam. The presence of many modes allows the summation of crosstalk power between individual modes to be converted to an integral expression. The result is applied to two parallel identical fibres, lossy fibres, fibres of unequal radius and arrays of fibres. We find that the light pipes must be nearly identical and touching for significant crosstalk.

The important results of this chapter are contained in Section 5.4 .

\subsection{INTRODUCTION}

The circular multimode optical fibre is a possibility for use in communications (Miller et al, 1973; Clarricoats, 1975). Multimode fibres, sometimes called light pipes, have $V \gg>1$ so that many bound modes can propagate, giving an almost uniform intensity within the fibre c.f. the modal patterns of small $V$ waveguides.

We have until now been concerned with coupling or crosstalk between a single pair of modes on neighbouring fibres. Our purpose in this chapter is to determine the total power transfer between 
neighbouring light pipes i.e. the summed effect of the coupling of many modes. The mathematical analysis is given in section 5.3 for two parallel light pipes and extended to include the effect of loss, fibres of unequal radius and arrays of fibres. In Section 5.4 we present results, including curves, showing the dependence of crosstalk on the system parameters.

There has been considerable recent success in using ray concepts to explain multimode-fibre phenomena (Kapany and Burke, 1972; Snyder, 1974). In the limit $V \gg 1$, modes can be thought of as being composed of families of rays all inclined at the same angle to the axis of the fibre. Ray theory has also been used to describe coupling between slab waveguides of arbitrary V (Kapany and Burke, 1972). We might expect therefore that it should be possible to analyse crosstalk between light pipes in terms of rays, and in fact several such analyses have appeared (Kapany, 1967; Cherin and Murphy, 1975). However these theories have several shortcomings in that they do not take account of phase or of cross-feedback between the waveguides, and difficulties arise in keeping track of ray paths, in incorporating the concept of total frustrated reflection at curved boundaries and in calculating the integrals. Because of these considerations, discussed in detail in Section 5.5, we choose to analyse the problem using mode theory; the basic theory is also given in terms of ray angles and parameters to enable geometric interpretations to be made.

When calculating the crosstalk due to more than one mode, we need to know the power distribution in the modes at the launching end of the fibre. We assume the fibre is excited by a focussed collimated beam of light. The analysis in section 5.3.2 shows that only. 
the cylindrically symmetric $\mathrm{HE}_{1 \mathrm{~m}}$ modes, formed by meridional rays only, are excited. Lens excitation is a common method of launching modes on multimode optical fibres. Possible extensions to incoherent and arbitrary excitations are discussed in section 5.5.

\subsection{MATHEMATICAL ANALYSIS}

\subsubsection{Two Parallel Identical Fibres}

We have shown in Chapter 3 that only coupling between like modes is significant when the fibres are identical (see also section 5.3.5). Therefore to determine total crosstalk power we need only sum the crosstalk power due to pairs of like modes. We assume that only one of the two light pipes in Fig. 5.Ia is illuminated. From (2.64), the crosstalk power $\mathrm{P}_{\mathrm{Ct}}^{\mathrm{q}}$ of the $\mathrm{q}^{\prime}$ th mode in the unilluminated light pipe is given by

$$
P_{C t}^{q}(z)=P_{i}^{q} \sin ^{2} \cdot C_{q} z
$$

where $P_{i}^{q}$ is the input $(z=0)$ power of the $q^{\prime}$ th mode in the illuminated light pipe, $z$ is the position along the axes of the light pipes and $\mathrm{C}_{\mathrm{q}}$ is the coupling coefficient between the $\mathrm{q}^{\prime}$ th modes. The total crosstalk power $\mathrm{P}_{\mathrm{Ct}}(z)$ in the unilluminated light pipe is

$$
P_{c t}(z)=2 \sum_{q=1}^{M} P_{i}^{q} \sin ^{2}\left(C_{q} z\right)
$$

and the total input power $P_{\text {in }}$ of the illuminated light pipe is

$$
P_{\text {in }}=2 \sum_{q=1}^{M} P_{i}^{q}
$$

where $M$ is the maximum number of bound $\mathrm{HE}_{1 \mathrm{~m}}$ modes that can propagate for given $V$. The factor 2 takes into account the two possible mode polarisations. When $V \gg 1$ we can use the asymptotic form (3.64) for 

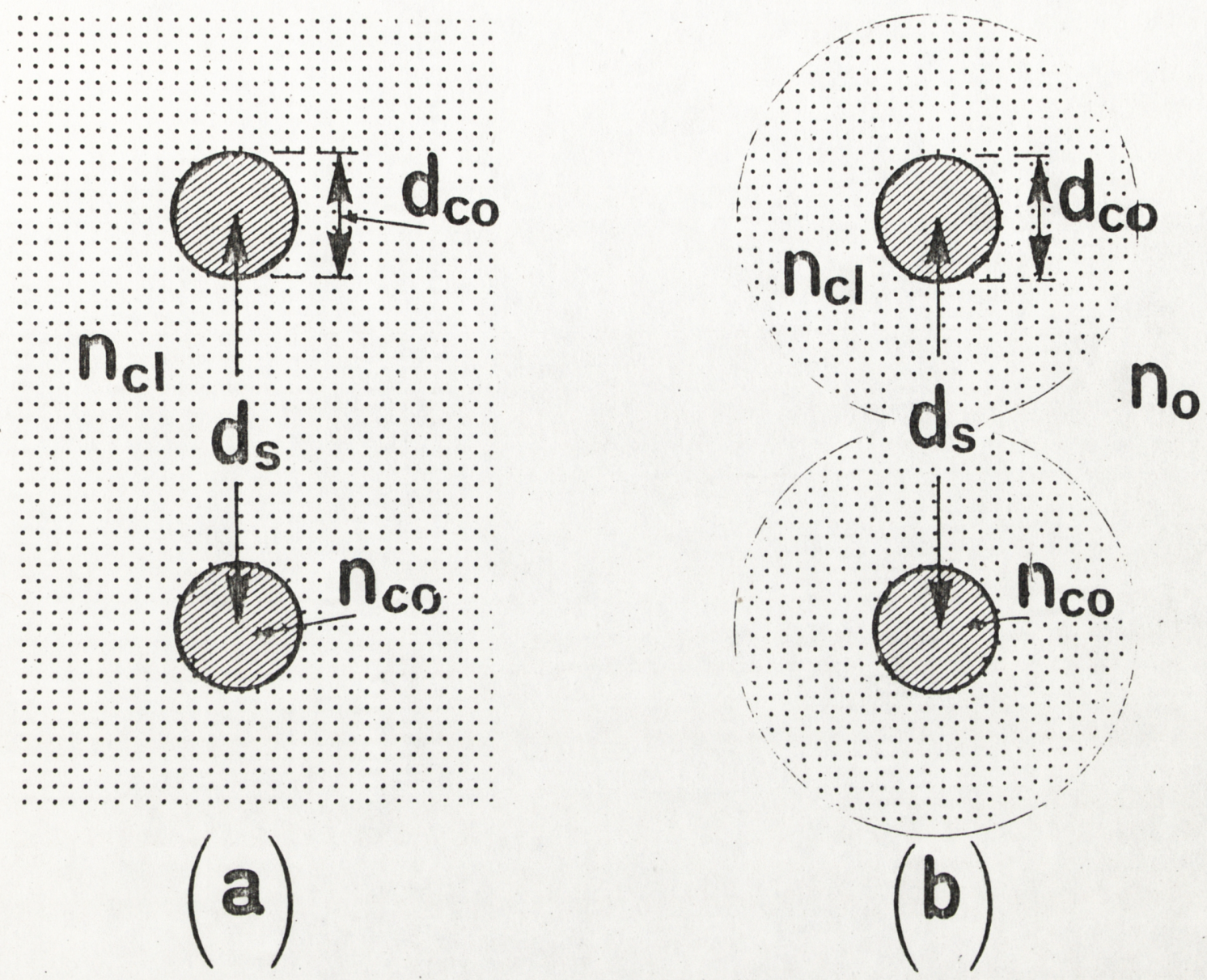

Fig. 5.1. (a) Two identical fibres embedded in an infinite medium of refractive index $\mathrm{n}_{\mathrm{Cl}}$, with a centre-tocentre separation $d_{S}$.

(b) Two cladded optical fibres in an external medium $n_{0}$. Because only coupling between meridional rays is important (see text), the situation in (a) represents a good approximation to that in (b), especially when $d_{S} \gg d_{C O}$. 
the coupling coefficient

$$
\left|C_{q}\right| \approx \frac{\sqrt{\delta}}{\rho} \frac{U_{q}^{2}}{v^{5 / 2}}\left(\frac{2}{\pi D}\right)^{\frac{3}{2}} e^{-V(D-2)}
$$

provided that the mode is not near cutoff. $D=d_{s} / \rho$ and the only mode dependence is in the factor $\mathrm{U}_{\mathrm{q}}$ i.e. $\mathrm{C}_{\mathrm{q}}=\mathrm{C}\left(\mathrm{U}_{\mathrm{q}}\right)$. Because $\mathrm{V} \gg \mathrm{I}, \mathrm{M} \gg \mathrm{I}$ and most of the eigenvalues $U_{q} \gg 1$. From the asymptotic expression for $\mathrm{J}_{0}(\mathrm{U})$, we find the $\mathrm{U}_{\mathrm{q}}$ 's are separated by approximately $\pi$ i.e. $\mathrm{U}_{\mathrm{n}}-\mathrm{U}_{\mathrm{n}-1} \approx \pi$. Therefore, provided that the modal input power is also only a function of mode through $U_{q}\left(P_{i}^{q}=P_{i}\left(U_{q}\right)\right)$, the sums $(5.2),(5.3)$ can be approximated as

$$
\begin{aligned}
P_{C t}(z) & \approx \frac{2}{\pi} \int_{0}^{U_{M}} P_{i}(U) \sin ^{2}[C(U) z] d U \\
P_{i n} & \approx \frac{2}{\pi} \int_{0}^{U_{M}} P_{i}(U) d U
\end{aligned}
$$

where $U_{M}$ is the maximum value of $U$, set by the excitation conditions. The assumption $p_{i}^{q}=P_{i}\left(U_{q}\right)$ is true for lens excitation and is probably a good approximation for any excitation where many bound modes are excited. If $\mathrm{P}_{i}(U) \approx 0$ for $U>U_{m}$, we can extend both integrals to $\infty$. We are justified in using ray concepts for waveguides when $\mathrm{V} \gg$ 1. In terms of the parameters of Fig. $5.1 \mathrm{a}$

$$
\begin{aligned}
& \mathrm{v}=\frac{\pi \mathrm{d}_{\mathrm{CO}} \mathrm{n}_{\mathrm{co}} \theta_{\mathrm{c}}}{\lambda} \\
& \theta_{\mathrm{c}} \approx \sin \theta_{\mathrm{C}}=\left[1-\mathrm{n}_{\mathrm{Cl}}^{2} / \mathrm{n}_{\mathrm{co}}^{2}\right]^{\frac{1}{2}}=\sqrt{\delta} .
\end{aligned}
$$

$\mathrm{n}_{\mathrm{Cl}} \approx \mathrm{n}_{\mathrm{CO}}$ is assumed so that $\theta_{\mathrm{C}} \ll 1, \theta_{\mathrm{C}}$ is the complement of the critical angle at the fibre boundary. We can relate $U$ to the angle $\theta$ between the ray direction and the axis of the light pipe by. (Snyder and Mitchell, 1974) 


$$
\begin{aligned}
\mathrm{U} & =\frac{\mathrm{V} \sin \theta}{\sin \theta_{\mathrm{C}}} \\
& \approx \frac{\mathrm{V} \theta}{\theta_{\mathrm{C}}} .
\end{aligned}
$$

Coupling between like $\mathrm{HE}_{\mathrm{lm}}$ modes only can be interpreted geometrically as meridional rays of one light guide coupling only to meridional rays of the other, where both rays have the same $\theta$. Therefore, to a good approximation we can replace the more realistic system of Fig. 5.lb by that in Fig. 5.1a.

\subsubsection{Lens Excitation}

The amount of crosstalk depends on the illumination conditions, which determine the distribution of power among the modes, and on the arrangement and physical parameters of the light pipes. We assume that a collimated beam of light is focussed onto the light pipe axis as shown in Fig. 5.2. We initially set $n_{0}=n_{C O}$ so that $\theta_{M}=\theta_{0}$. The electric field $\mathrm{E}$ at the focal plane is that due to Fraunhofer diffraction by a circular aperture of diameter $d_{\ell}$ (Jackson, 1962; Marcuse, 1972)

$$
\begin{aligned}
E & =E_{O} \frac{J_{1}(B R)}{B R} \\
B & =\frac{2 \pi \rho}{\lambda} \frac{d_{l}}{2 f} \\
& =\frac{V}{\sqrt{\delta}} \frac{d_{l}}{2 f} \\
& =\frac{V \sin \theta_{M}}{\sin \theta_{C}} \\
& =\frac{V \sin \theta_{M}}{\sin \theta_{C}} \\
& =U_{M},
\end{aligned}
$$

where $R=r / \rho, 2 \rho=d_{C O^{\prime}}\left|E_{O}\right|^{2}$ is proportional to the input power and $r$ is 


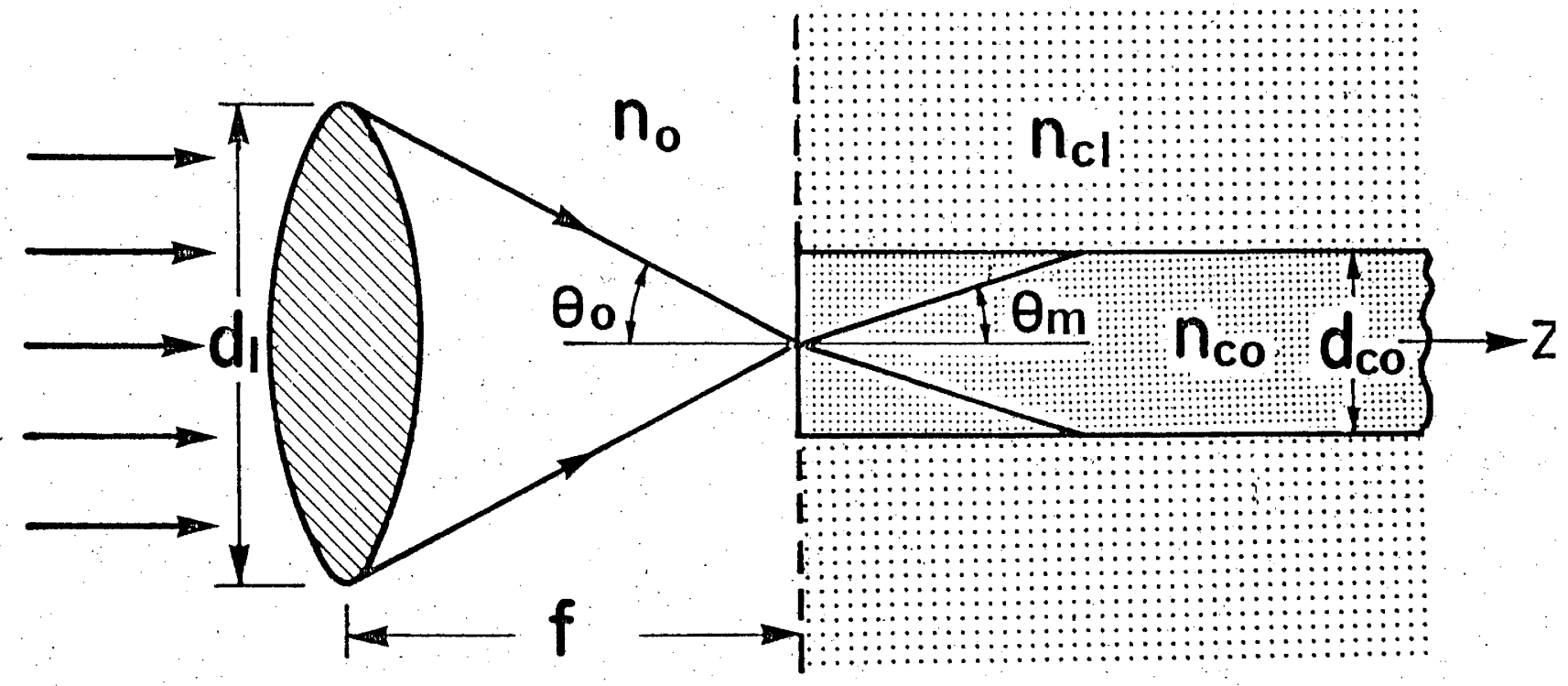

FP

Fig. 5.2. Illumination of an optical fibre. The core has a circular cross-section of diameter $\mathrm{d}_{\mathrm{CO}}(=2 \rho)$ and refractive index $\mathrm{n}_{\mathrm{CO}} \cdot \mathrm{n}_{\mathrm{Cl}}$ and $\mathrm{n}_{\mathrm{O}}$ are the refractive indexes of the cladding and of the medium external to the fibre. The lens of diameter $d_{1}$ and focal length $f$, is illuminated by a highly collimated on-axis beam with azimuthal symmetry. FP is the focal plane of the lens. 
the radial coordinate at $z=0$. We have neglected a quadratic phase term $\exp \left[2 \pi i r^{2} / 2 f \lambda\right]$ as it is nearly unity when $|\underline{E}|$ is significant (Goodman, 1968; Born and Wolf, 1970).

The power $P_{i}^{q}$ of the $q^{\prime}$ th mode is (Snyder, 1969b, 1974a)

$$
\mathrm{P}_{i}^{\mathrm{q}}=\left|\int_{\mathrm{A}_{C O}} \underline{E} \times \underline{\mathrm{h}}_{-\mathrm{q}}^{*} \cdot \underline{\hat{z}} \mathrm{dA}\right|^{2} /\left|\int_{A_{\infty}} \underline{\mathrm{e}}_{\mathrm{q}} \times \underline{\mathrm{h}}_{-\mathrm{q}}^{*} \cdot \underline{\hat{z}} \mathrm{dA}\right|^{2}
$$

where $e^{\prime} q^{\prime}-q$ are the modal electric and magnetic fields for the $q^{\prime}$ th mode, $\hat{z}$ is a unit vector, $A_{\infty}$ is the infinite cross-section and $A_{c o}$ the cross-sectional area of the core. Substituting the modal fields of Section 3.3 into (5.13) shows that only the HE ${ }_{1 \mathrm{~m}}$ modes are excited and that the power of these modes is

$$
\begin{aligned}
& P_{i}^{q} \approx 2 \pi^{2}\left(\frac{\varepsilon_{0}}{\mu}\right)^{\frac{1}{2}}\left(\frac{E_{0} \rho}{B}\right)^{2} U_{q} I_{q} \\
& I_{q}=B \int_{0}^{I} J_{0}\left(U_{q} R\right) J_{1}(B R) d R
\end{aligned}
$$

where $v \gg U_{q}$ is assumed, $\varepsilon_{0}$ is the dielectric constant of the medium to the left of the focal plane and $\mu$ is its permeability. This integral has no analytic solution; however as $\mathrm{V} \gg I_{1} \mathrm{~J}_{1}(\mathrm{~B})<<1$ so that the upper limit of integration can be taken to be infinite, giving $I_{q}=1$ for $\mathrm{B}>\mathrm{U}_{\mathrm{q}}$ and $\mathrm{I}_{\mathrm{q}}=0$ for $\mathrm{B}<\mathrm{U}_{\mathrm{q}}$. In deriving (5.14) we have used the $\mathrm{V}>\mathrm{U}_{\mathrm{q}}$ eigenvalue equation $\mathrm{V} \mathrm{J} \mathrm{J}_{0}\left(\mathrm{U}_{\mathrm{q}}\right) \approx \mathrm{U}_{\mathrm{q}} \mathrm{J}_{1}\left(\mathrm{U}_{\mathrm{q}}\right)$, and the fact that $J_{1}\left(U_{q}\right) \approx\left(\frac{2}{\pi U_{q}}\right)^{\frac{1}{2}}$ when $J_{0}\left(U_{q}\right) \approx 0$.

In summary, $P_{i}\left(U_{q}\right)=a U_{q}$ (a constant) when $U_{q}<U_{M}$ and $P_{i}\left(U_{q}\right)=0$ when $U_{q}>U_{M}$. These results assume that the mode is far from cutoff, which is satisfied approximately when $U_{M}<V / 3$.

In terms of rays, the amount of light that enters the light guide is characterised by the radiant intensity distribution $D(\theta)$ i.e. 
the power per unit solid angle. For the lens excitation discussed above, $D(\theta)=1$ for $0<\theta<\theta_{M}$ and 0 for $\theta>\theta_{M}$. The total power $P_{i}=2 \pi \int_{0}^{\pi / 2} D(\theta) \sin \theta \cdot d \theta$.

The above results have been derived assuming $\theta_{M}=\theta_{0}$ but can be generalised for the case $\theta_{0} \ll 1$ by using Snell's law, giving

$$
\theta_{\mathrm{M}} \approx \frac{\mathrm{n}_{\mathrm{O}} \theta_{\mathrm{O}}}{\mathrm{n}_{\mathrm{CO}}} \approx \frac{\mathrm{n}_{\mathrm{O}} \mathrm{d} \ell}{2 \mathrm{n}_{\mathrm{CO}} \mathrm{f}}
$$

where $\mathrm{n}_{\mathrm{O}}<\mathrm{n}_{\mathrm{CO}}$. Some of the incident energy is reflected from the $\mathrm{n}_{\mathrm{o}}-$ fibre interface but the reflected energy is generally small and when $\mathrm{n}_{\mathrm{o}} \approx_{\mathrm{n}} \mathrm{n}^{\prime}$ approximately independent of $\theta$. The reflection coefficient is then a common factor in the expressions for $\mathrm{P}_{\mathrm{Ct}} \mathrm{t} \mathrm{P}_{\text {in }}$ and is not required for the analysis (Snyder et al, 1973).

In practice $D(\theta)$ often has a gaussian shape due to the combined effect of imperfect optics and a non-uniform beam illuminating the lens i.e.

$$
D(\theta)=e^{-\left(\frac{\tau \theta}{\theta_{M}}\right)^{2}}
$$

where $\tau$ is a constant determined by the illumination conditions. We generalise our results to include this non-ideal situation by putting

$$
\mathrm{P}_{\mathrm{i}}(\mathrm{U})=\dot{\mathrm{U}} \mathrm{D}(\mathrm{U})
$$

For the ideal optical system

$$
D(U)= \begin{cases}D_{O} & U<U_{M} \\ 0 & \text { otherwise }\end{cases}
$$

For the imperfect optical system

$$
D(U)=D_{0} e^{-\left(\frac{T U}{U_{M}}\right)^{2}}
$$

where $D_{0}$ is a constant. 


\subsubsection{Crosstalk Power}

Substituting the expressions for the coupling coefficient (5.4) and the initial power distribution (5.18) into the integral approximations $(5.5),(5.6)$, we find

$$
\frac{P_{C t}}{P_{\text {in }}}=\frac{1}{2}\left[1-\frac{\sin L}{I}\right]
$$

for the ideal optical system characterised by $(5.19)$. If $\tau^{2}>1.5$ in $(5.20), \mathrm{D}(\mathrm{U}) \approx 0$ for $\mathrm{U}>_{\mathrm{U}_{\mathrm{M}}}$ so that the upper limit of the integrals in (5.5), (5.6) can be extended to infinity, giving for the imperfect optical system

$$
\frac{P_{\text {Ct }}}{P_{\text {in }}}=\frac{1}{2}\left[\frac{L^{2}}{L^{2}+\tau^{4}}\right]
$$

L is a normalised length given by

$$
\begin{aligned}
L & =\frac{2 \sqrt{\delta} z}{\rho_{C O}}\left(\frac{\mathrm{U}}{\mathrm{V}}\right)^{2}\left(\frac{2}{\pi V D}\right)^{\frac{1}{2}} \mathrm{e}^{-\mathrm{V}(\mathrm{D}-2)} \\
& =\frac{2 \theta_{C} z}{\rho_{C O}}\left(\frac{\theta_{\mathrm{M}}}{\theta_{C}}\right)^{2}\left(\frac{2}{\pi V D}\right)^{\frac{1}{2}} \mathrm{e}^{-\mathrm{V}(\mathrm{D}-2)}
\end{aligned}
$$

Equation (5.22) is an approximation to the exact $\int_{0}^{U_{m}}$, valid for large $\tau^{2}$. The integrals can however be calculated exactly for arbitrary. $\tau$ giving

$$
\frac{P_{\text {ct }}}{P_{\text {in }}}=\frac{1}{2}\left\{1-\frac{\tau^{2}}{L^{2}+\tau^{4}}\left[\frac{\tau^{2}+e^{-\tau^{2}}\left(L \sin L-\tau^{2} \cos L\right)}{1-e^{-\tau^{2}}}\right]\right\}
$$

This expression is a general one including both the perfect optics case $(\tau \rightarrow 0)$ and the imperfect optics case $\left(\tau^{2} \gg 1\right)$

The behaviour of the expressions (5.21) (integral form) and (5.2)/(5.3) (summation form) are shown as a function of L in Fig. 5.3 


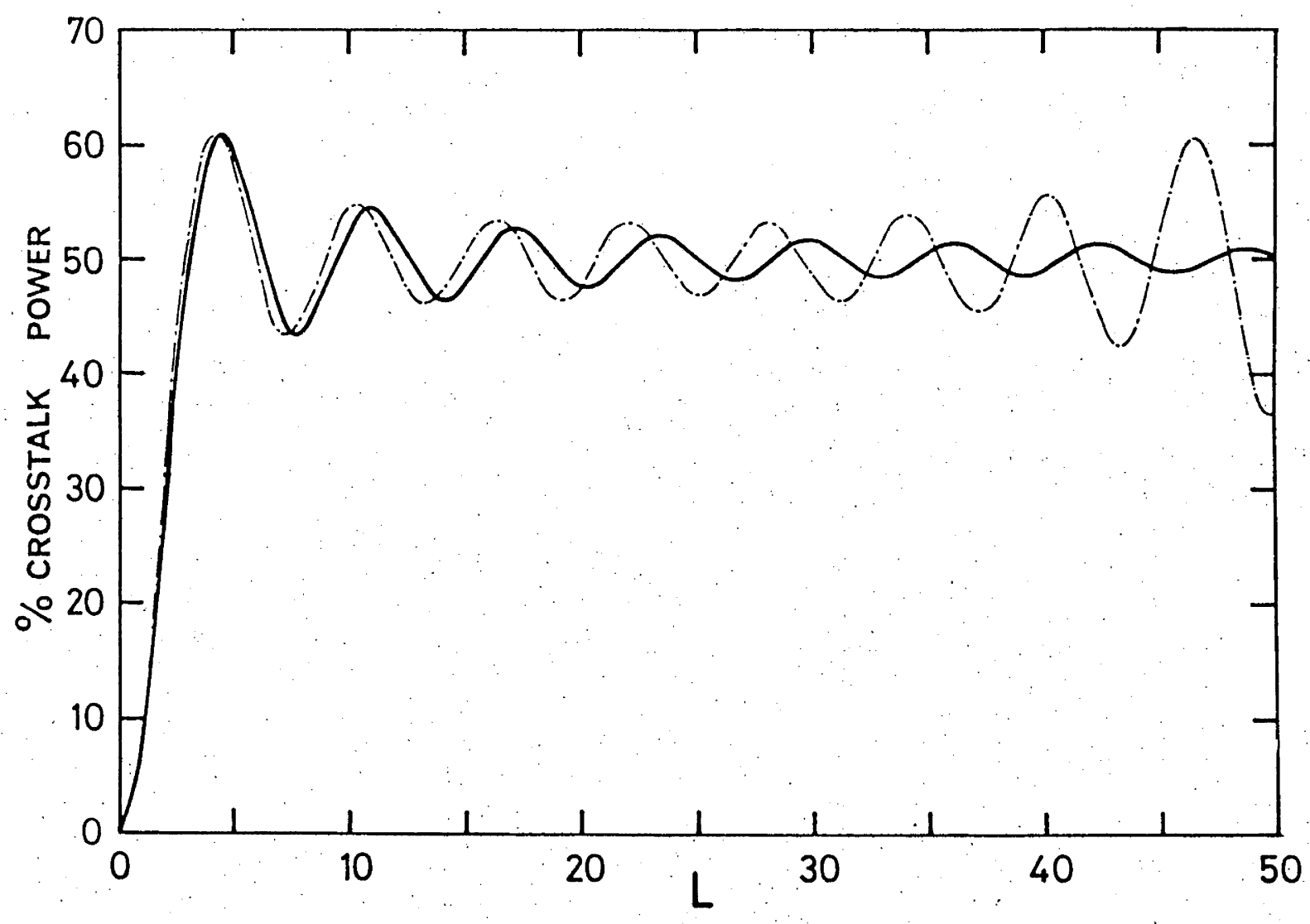

Fig. 5.3 Comparison of the exact mode-sum $(-\rightarrow-\rightarrow$ for ${ }^{P_{C t}} / P_{\text {in }}$ with $U_{M}=50$, with the integral approximation $(\longrightarrow$ for a perfect-optics system. The integral is a function of $L$ only, whereas the sum is a function of $\mathrm{L}$ and $\mathrm{U}_{\mathrm{M}}$ which is a measure of the number of modes contributing to the sum. When $\mathrm{U}_{\mathrm{M}} \sim 500$, the sum curve is too close to the integral curve to show here. 
for the perfect optical system. The integral form is a function only of $L$, whereas the summation is a function of $L$ and of the number of modes i.e. of $\mathrm{U}_{\mathrm{M}}$. The integral provides a good approximation to the sum for small $L$ even with only 16 terms $\left(U_{M} \approx 50\right)$, but the sum diverges from the integral at $\mathrm{L} \approx \mathrm{U}_{\mathrm{M}}$. As the number of modes is increased the integral approximation becomes better, and with $\mathrm{U}_{\mathrm{M}}=500$ (159 terms), the sum and integral curves in Fig. 5.3 are indistinguishable. However there is still a divergence of the sum and integral at $L \approx 500$. The amplitude of the divergence appears to decrease as more modes are included in the sum. The behaviour of the sum is due to the fact that it is a sum of many $\sin ^{2}$ terms each with a different period. The sum then also has a (much longer) period and oscillates about a mean of 0.5 . Provided the individual term periods are randomly distributed (modulus $2 \pi$ ), the sum period should tend to $\infty$ and its amplitude about 0.5 tend to 0 as the number of terms is increased.

The integral approximation for the imperfect case is better than that for the perfect optics, but still shows the divergence at $L \approx U_{M}$. The error due to extending the integration limit from $\mathrm{U}_{\mathrm{M}}$ to $\infty$ is significant when $\tau^{2} \sim 1.5$ but decreases rapidly as $\tau^{2}$ increases.

In practical situations it is probably almost impossible to achieve such large values of $\mathrm{L}$ in perfectly uniform fibres, so that the divergence of the sum and integral is of theoretical interest only.

\subsubsection{Absorption Losses}

When the core and cladding are lossy (Snyder, 1972; Chapter 6), the modal crosstalk power for mode $q$ is given by

$$
P_{c t}^{q}(z)=P_{i}^{q} \sin ^{2}\left(C_{q} z\right) e^{-\alpha} q^{z}
$$


where (Snyder, 1974; 1972a, 1969a)

$$
\begin{gathered}
\alpha_{\mathrm{q}} \approx \alpha_{\mathrm{CO}}+\left(\alpha_{\mathrm{Cl}}-\alpha_{\mathrm{CO}}\right)\left(\mathrm{U}_{\mathrm{q}}^{2} / \mathrm{v}^{3}\right) \\
\approx \alpha_{\mathrm{CO}}+\left(\frac{\alpha_{\mathrm{Cl}}-\alpha_{\mathrm{CO}}}{\mathrm{v}}\right)\left(\frac{\theta}{\theta_{\mathrm{C}}}\right)^{2}
\end{gathered}
$$

$\alpha_{C O}, \alpha_{C l}$ are the core and cladding absorption coefficients and we have again assumed $\mathrm{V} \gg \mathrm{U}_{\mathrm{q}}$ or $\theta \ll \theta_{\mathrm{C}}$. If the core and cladding have approximately the same bulk power attenuation coefficient $\alpha$, we need only multiply our results for $P_{c t}$ by $e^{-\alpha z}$ to account for absorption losses. $\alpha$ is independent of angle $\theta$ when $\theta \ll 1$.

When $\alpha_{c l}$ is considerably greater than $\alpha_{C O}$ ' the loss term introduces an exp $\left(\mathrm{bu}^{2}\right)$ where $b$ is a constant. This can be included in the gaussian term in $\mathrm{P}_{\mathrm{Ct}}$ of the imperfect optical case to give

$$
\frac{P_{C t}}{P_{\text {in }}}=\frac{1}{2}\left[\frac{L^{2}+\gamma^{2}\left(\tau^{2}+\gamma^{2}\right)}{L^{2}+\left(\tau^{2}+\gamma^{2}\right)^{2}}\right] e^{-\alpha_{C O} z}
$$

where

$$
\gamma^{2}=\left(\alpha_{c l}-\alpha_{c o}\right) z\left(\frac{U_{M}^{2}}{v^{3}}\right)
$$

and $\tau^{2} \gtrsim 1.5$. If $\tau^{2} \lesssim 1.5$ the exact integral, similar to that leading to $(5.24)$ must be calculated, giving

$$
\begin{aligned}
\frac{P_{C t}}{P_{\text {in }}}=\frac{1}{2}\left\{1-\frac{\tau^{2}}{L^{2}+\left(\tau^{2}+\gamma^{2}\right)^{2}}\right. & {\left[\frac{\tau^{2}+\gamma^{2}+e^{-\left(\tau^{2}+\gamma^{2}\right)}\left(L \sin L-\left(\tau^{2}+\gamma^{2}\right) \cos L\right)}{\left.\left.1-e^{-\tau^{2}}\right]\right\}}\right.} \\
& \times e^{-\alpha O^{z}}
\end{aligned}
$$

For the perfect optical system, there is a term exp $\left\{-\left(\frac{\gamma U}{U_{M}}\right)^{2}\right\}$ in the integral for $P_{c t}$ and as $\gamma^{2}<1$, we cannot extend the integral limit to $\infty$. Again we calculate the exact integral, giving 


$$
\frac{\mathrm{P}_{\text {Ct }}}{\mathrm{P}_{\text {in }}}=\frac{1}{2}\left\{1-\frac{1}{\mathrm{~L}^{2}+\gamma^{4}}\left[\gamma^{2}+\mathrm{e}^{-\gamma^{2}}\left(\mathrm{~L} \sin \mathrm{L}-\gamma^{2} \cos \mathrm{L}\right)\right]\right\} \mathrm{e}^{-\alpha_{C O} z}
$$

\subsubsection{Two Parallel Fibres with Unequal Radii}

When the light pipes differ in radius by an amount $\Delta \rho$, the crosstalk power is given by $(2.64)$ as

$$
P_{C t}=2 F \sum_{q=1}^{M} P_{i}^{q} \sin ^{2}\left(C_{q} z / \sqrt{F}\right)
$$

where

$$
F=\left[1+\left(\frac{\Delta \beta}{2 C_{q}}\right)^{2}\right]^{-1}
$$

From (3.26),

$$
\Delta \beta_{\mathrm{q}} \approx \sqrt{\delta}\left(\frac{\Delta \rho}{\rho^{2}}\right) \frac{\mathrm{U}^{2}}{\mathrm{~V}}
$$

so that using (5.4)

$$
\frac{\Delta B_{\mathrm{q}}}{2 C_{\mathrm{q}}} \approx \frac{1}{2}\left(\frac{\pi \mathrm{DV}^{3}}{2}\right)^{\frac{3}{2}}\left(\frac{\Delta \rho}{\rho}\right) \mathrm{e}^{\mathrm{V}(\mathrm{D}-2)}
$$

which is independent of $q$. Therefore the results $(5.21)-(5.23)$ hold with $\mathrm{L}$ replaced by $\mathrm{L} / \sqrt{\mathrm{F}}$ and $\mathrm{P}_{\mathrm{ct}} / \mathrm{P}_{\text {in }}$ multiplied by $\mathrm{F}$.

A further consideration when the light pipe radii are not equal is coupling between unlike modes. We have neglected cross-mode coupling in our analysis because on identical light pipes it is negligibly small in comparison with like-mode coupling.. Cross-mode coupling is also described by (5.32) - (5.35) but with $\frac{\Delta \rho}{\rho}$ replaced by $\frac{\Delta \mathrm{U}}{\mathrm{U}}$. When these are comparable in magnitude, cross-mode coupling may be as important as like-mode coupling but in both cases the crosstalk power is generally very small. 


\subsubsection{Arrays of Fibres}

The theory can be extended to arrays of light pipes following Snyder (1972) and Section 3.6. Consider as an example the hexagonal array of Fig. 5.4. The system of Fig. 5.4b can be approximated by that of Fig. 5.4a because we consider only meridional rays. If the light pipes are identical and the centre light pipe is illuminated by the lens, the total crosstalk power in the six surrounding light pipes is given by

$$
P_{C t}(z)=2 \sum_{q=1}^{M}(6 / 7) P_{i}^{q} \sin ^{2}\left(\sqrt{7} C_{q} z\right)
$$

Therefore $(5.21)-(5.23)$ hold with $\mathrm{L}$ replaced by $\sqrt{7} \mathrm{~L}$ and $\mathrm{P}_{\text {ct }} / \mathrm{P}_{\text {in }}$ multiplied by 6/7. This can be extended to the general case of $n$ identical fibres equidistant from a central one, but for $n \neq 6$, the coupling coefficient $C_{s}$ between any two neighbouring surrounding fibres is different to that between the centre and a surrounding fibre $C_{C^{\prime}}$ so that $F$ is given by $F=\left[1+\frac{1}{n}\left(\frac{C_{S}}{C_{C}}\right)^{2}\right]^{-1}$. The theory also allows the centre fibre to differ in radius from the surrounding ones.

\subsection{RESULTS}

\subsubsection{Identical Fibres}

We show in section 5.3 .3 that the crosstalk power for the idealised or perfect optical system is given by

$$
\frac{\mathrm{P}_{\text {ct }}}{\mathrm{P}_{\text {in }}} \approx \frac{1}{2}\left[1-\frac{\sin \mathrm{L}}{\mathrm{L}}\right] \ldots
$$

and for the imperfect optical system characterised by a parameter $\tau$, by

$$
\frac{P_{C t}}{P_{\text {in }}} \approx \frac{1}{2}\left[\frac{L^{2}}{L^{2}+T^{4}}\right]
$$

where the normalised length $L$ is given by 


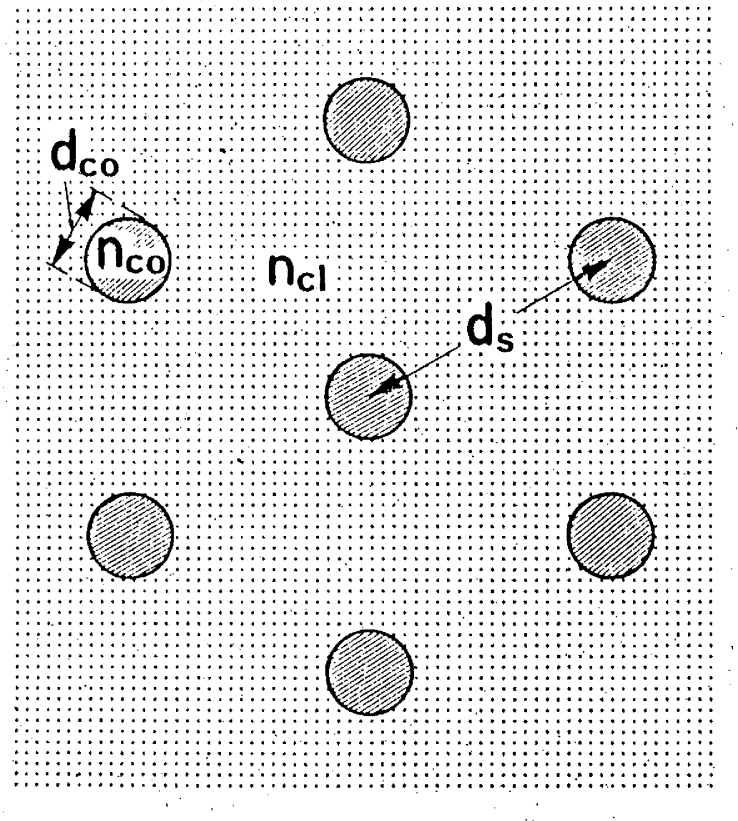

(a)

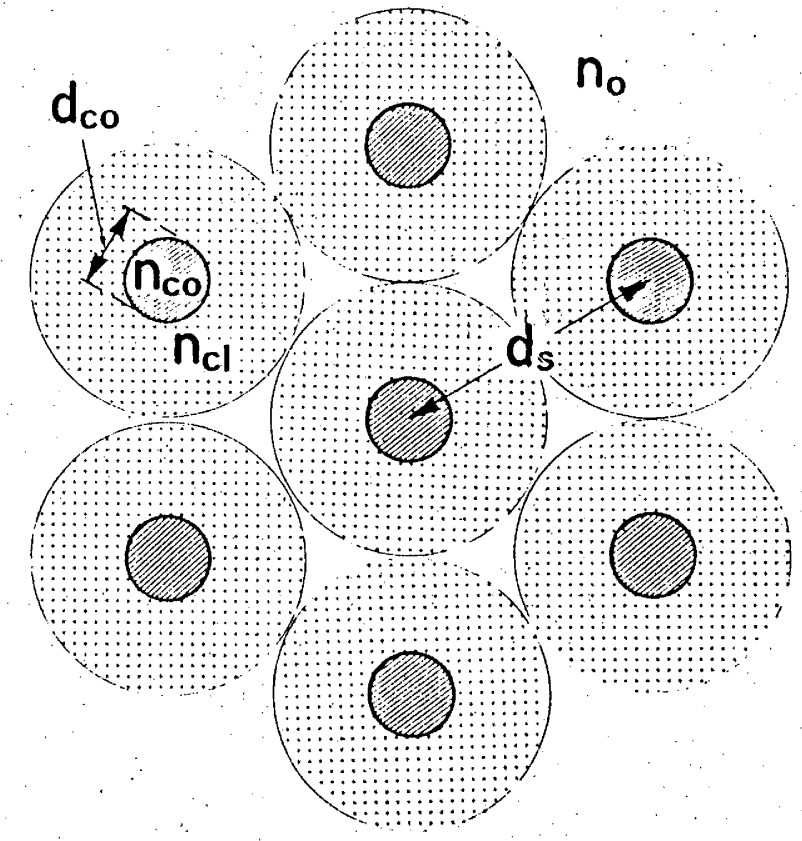

(b)

Fig. 5.4. Hexagonal array of identical fibres

(a) Fibres of refractive index $n_{C O}$ embedded in an infinite medium of refractive index $\mathrm{n}_{\mathrm{Cl}}$ :

(b) Closely packed optical fibres. As in Fig. 5.1, (a) represents a good approximation to (b) : 


$$
\begin{aligned}
L & =\frac{2 \sqrt{\delta} z}{\rho}\left(\frac{U}{V}\right)^{2}\left(\frac{2}{\pi V D}\right)^{\frac{1}{2}} e^{-V(D-2)} \\
& =\frac{2 \theta_{C} z}{\rho}\left(\frac{{ }_{M}}{\theta_{C}}\right)^{2}\left(\frac{2}{\pi V D}\right)^{\frac{1}{2}} e^{-V(D-2)}
\end{aligned}
$$

where $D=d_{S} / \rho$ (Fig. 5.1), $\theta_{C}$ is the complement of the critical angle and $\theta_{\mathrm{M}}$ is determined by the illumination conditions. The result $(5.38)$ is valid for $\tau^{2} \gtrsim 1.5$; if this is not the case, $(5.24)$ must be used instead.

In the idealised optics case we need only specify $L$ to determine $P_{C t}$ ' whereas with imperfect optics we must in addition specify the degree of imperfection $\tau$. Figure 5.5 shows the crosstalk power for both the perfect and imperfect optics cases as a function of $\mathrm{L}$, calculated from $(5.37),(5.38)$. We emphasize that for the ideal optics case, this one curve can be used for all systems. We have plotted the crosstalk power for the imperfect case as a function of $L$ and $\tau^{2}$ to show the effect of different degrees of imperfection. However by plotting $\mathrm{P}_{C t} / \mathrm{P}$ in as a function of $\mathrm{L} / \tau^{2}$ (Fig. 5.6), we have a universal curve $\left(\tau^{2} \gtrsim 1.5\right)$ for the imperfect optics case as well.

The total crosstalk is made up of a sum of contributions from pairs of coupled modes, each with a different coupling coefficient and therefore a different rate of power transfer. The higher-order modes, which are excited with the greatest power by the ideal lens system, have the fastest rate of power transfer and are responsible for the overshoot in the ideal optics curve in Fig. 5.5. When the excitation power curve is rounded off, as with the gaussian excitation in the imperfect optics case, less power is launched into the higher-order modes and no overshoot occurs. As $\tau^{2}$ is decreased, oscillations appear in the imperfect optics curves and as $\tau^{2} \rightarrow 0$ we recover the perfect 


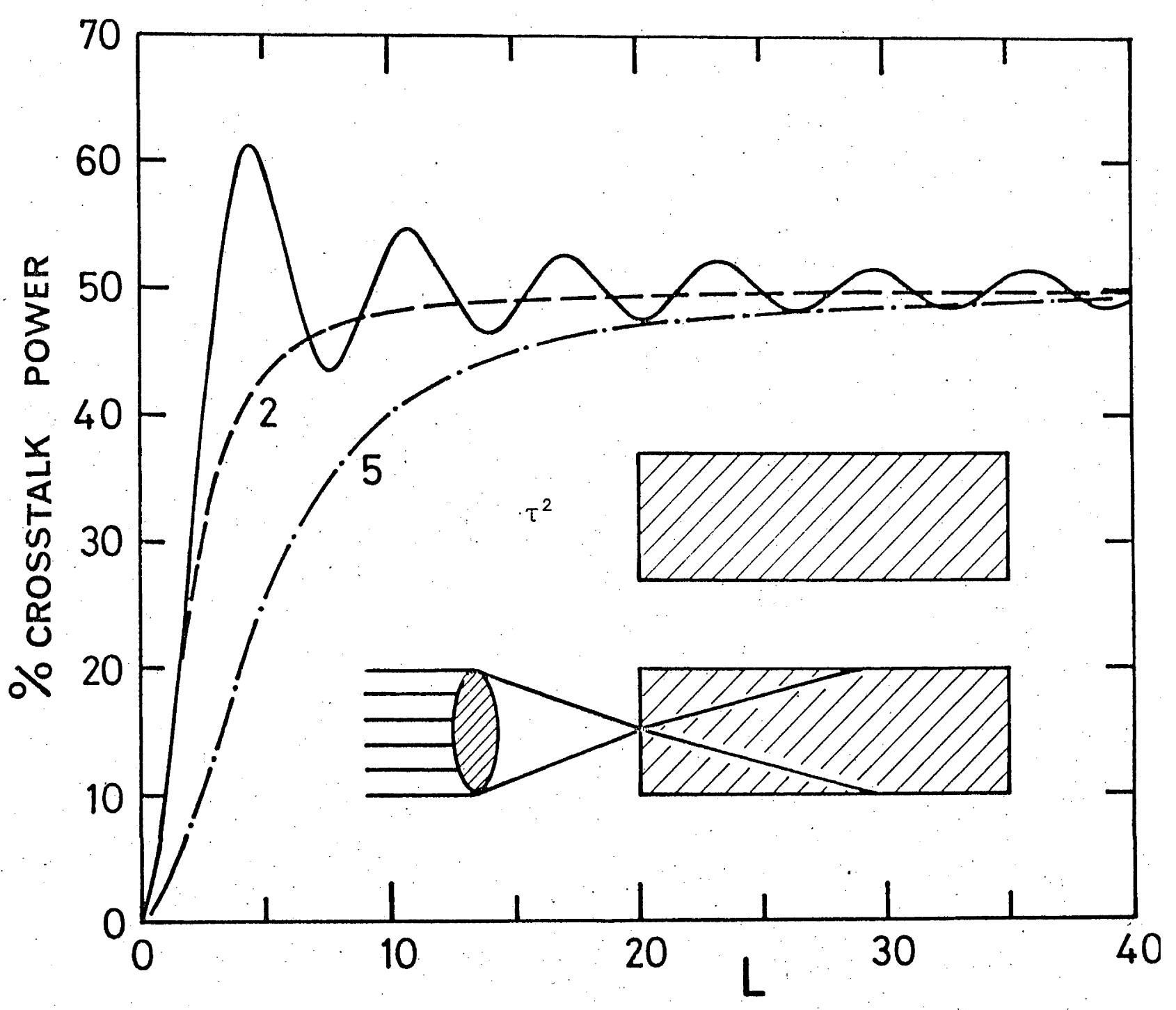

Fig. 5.5. Percent crosstalk power (power in the unilluminated fibre) between two identical parallel fibres, when one is illuminated as in Fig. 5.2 with unity total power $\left(P_{\text {in }}=1\right)$. The normalised length $L$ is given by (5.39). The ideal-optics case $(\longrightarrow$ ) and the imperfect-optics case with $\tau^{2}=2(--\longrightarrow)$ and $\tau^{2}=5$ (-. ) are shown. 


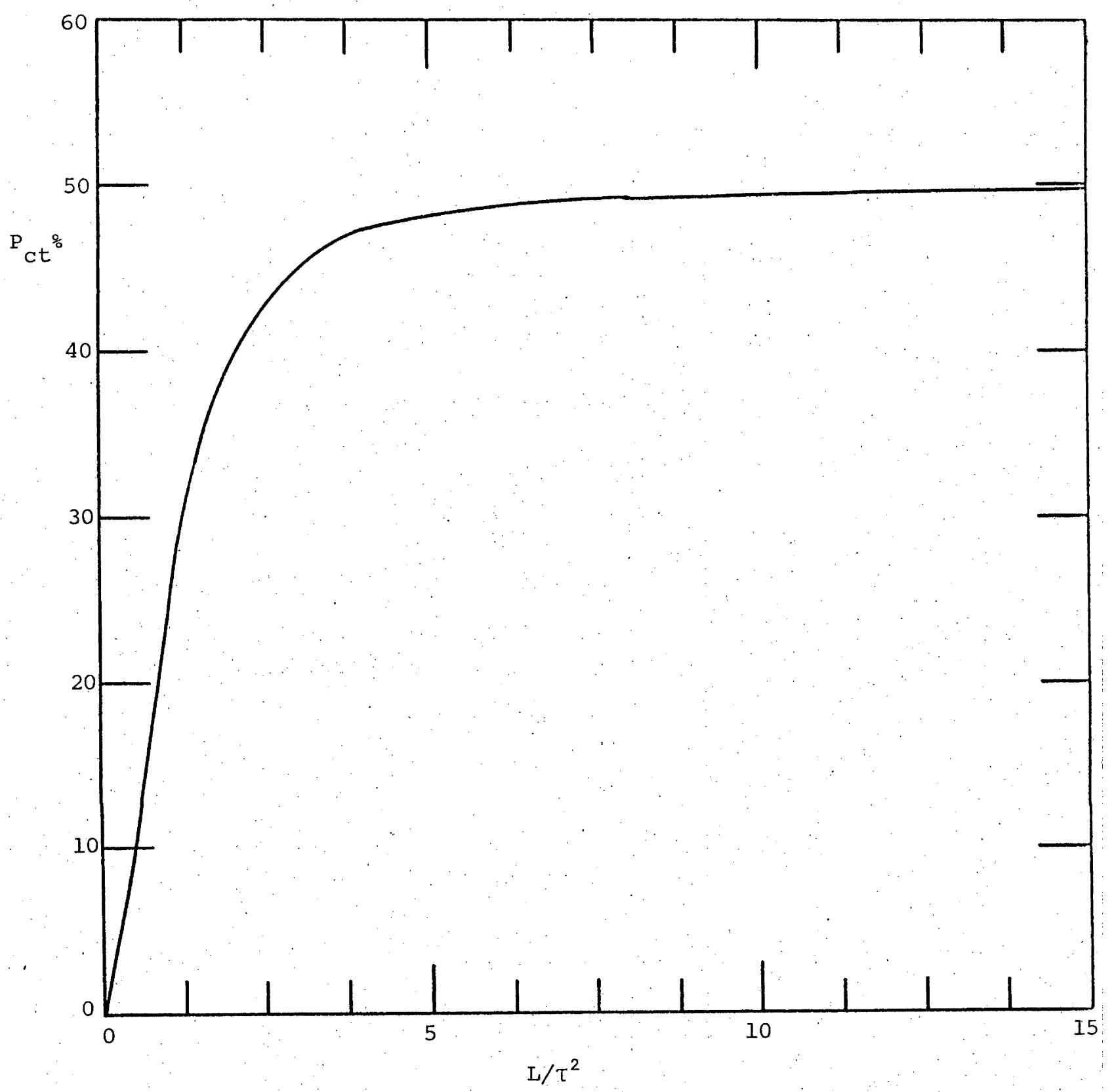

Fig. 5.6. Percent crosstalk power between two identical parallel fibres, when one is illuminated as in Fig. 5.2 with unity total power $\left(\mathrm{P}_{\text {in }}=1\right)$. The excitation assumes imperfect optics characterised by the parameter $\tau$. 
optics limit from (5.24). It is only in the two limits $\tau=0, \tau^{2} \gtrsim 1.5$ that we can give universal curves for the crosstalk power.

As a specific example, Fig. 5.7 shows the crosstalk power $\mathrm{P}_{\mathrm{Ct}} / \mathrm{P}_{\text {in }}$ for light pipes with $\theta_{\mathrm{C}}=11.5^{\circ}$ and $\rho=50 \mu$ as a function of length $z$. Unless the fibres are nearly touching, a rather protracted length is required for significant power transfer. The greater $v$, the greater this length. Because the crosstalk is acutely sensitive to the light pipes being parallel and identical, the theory is only useful in practical situations when great lengths are not required. Any deviation from identical fibres along their length will cause a marked drop in power transfer (Section 5.4:3).

\subsubsection{Absorption Losses}

The expressions for $\mathrm{P}_{C t} / \mathrm{P}$ in including absorption are given by (5.31) for the perfect optics case, and by $(5.28)\left(\tau^{2}+\gamma^{2} \gtrsim 1.5\right)$ or $(5.30)\left(\tau^{2}+\gamma^{2} \lesssim 1.5\right)$ for the imperfect optics case.

We find that even when the cladding loss is large $\left(\alpha_{\mathrm{cl}} \mathrm{z} \approx 90\right)$ it has very little effect on the crosstalk in the imperfect optics case, especially when $\tau^{2} \gtrsim 3$. To a good approximation the crosstalk power is $\frac{\mathrm{P}_{\text {Ct }}}{\mathrm{P}_{\text {in }}} \mathrm{e}^{-\alpha_{\mathrm{CO}^{2}}} \cdot$ Physically this is because the cladding loss effects the higher-order modes most, and with large enough $\tau^{2}$ these modes are excited with little power. Mathematically the difference is that between a curve with parameter $\tau^{2}$ and one with $\tau^{2}+\gamma^{2} . \gamma^{2}=1$ represents an extreme case, so that the difference is not great in general.

We would expect a larger effect for the perfect optics case because the higher order modes are excited with significant power. Figure 5.8 shows however that because $\gamma^{2} \lesssim 1$, the effect is not great and results mainly in reduced overshoot of the curve. The curve is for 


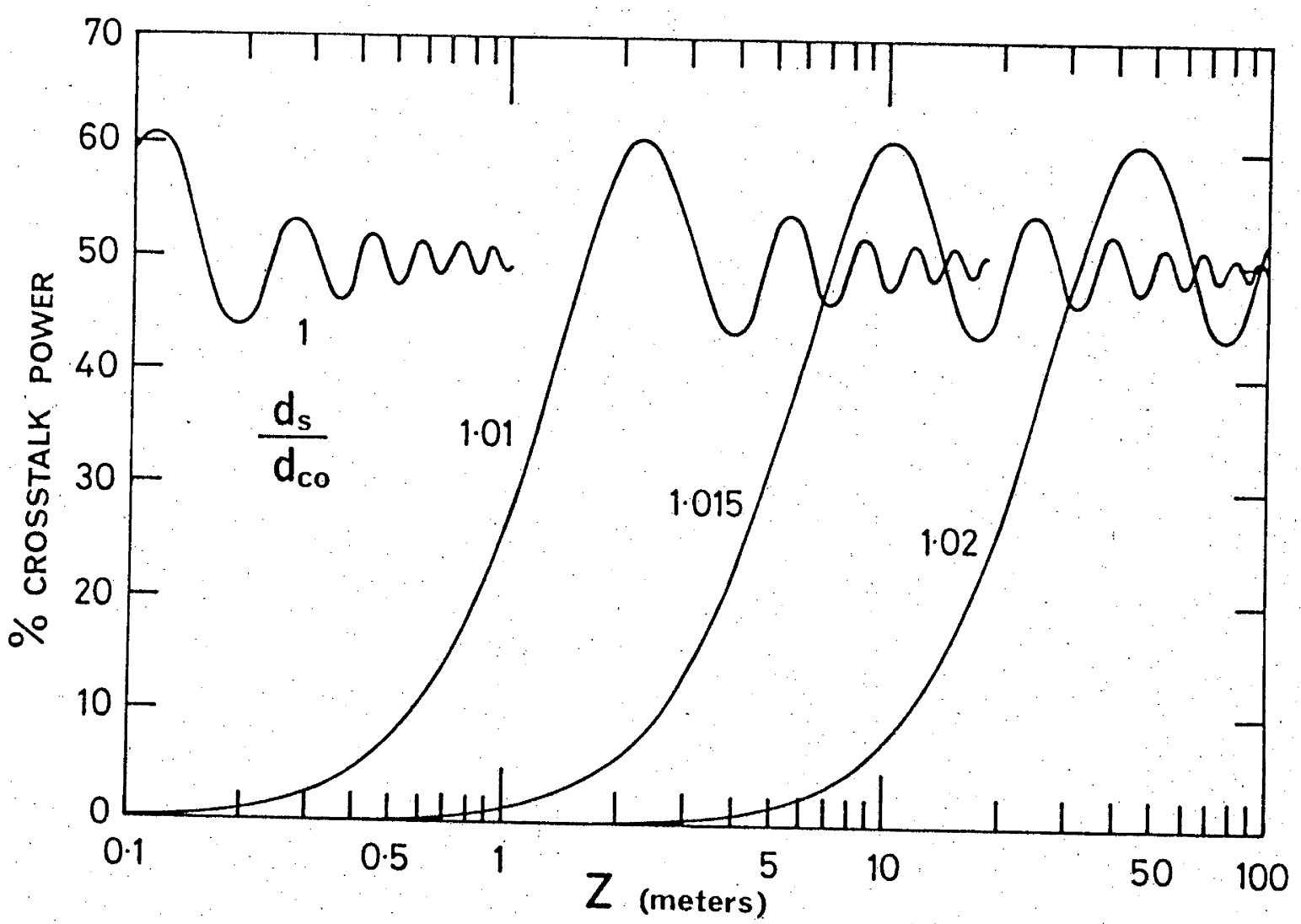

Fig. 5.7. Percent crosstalk power at position $z$ along the unilluminated fibre. $V=150, \theta_{C}=0.2$, $\mathrm{d}_{\mathrm{CO}}=2 \rho=100 \mu, \mathrm{U}_{\mathrm{M}}=50$. Excitation by a perfect optical system. The curves for $\mathrm{d}_{\mathrm{s}} / \mathrm{d}_{\mathrm{CO}}=1,1.01$ have been truncated to avoid confusion with the other curves. 


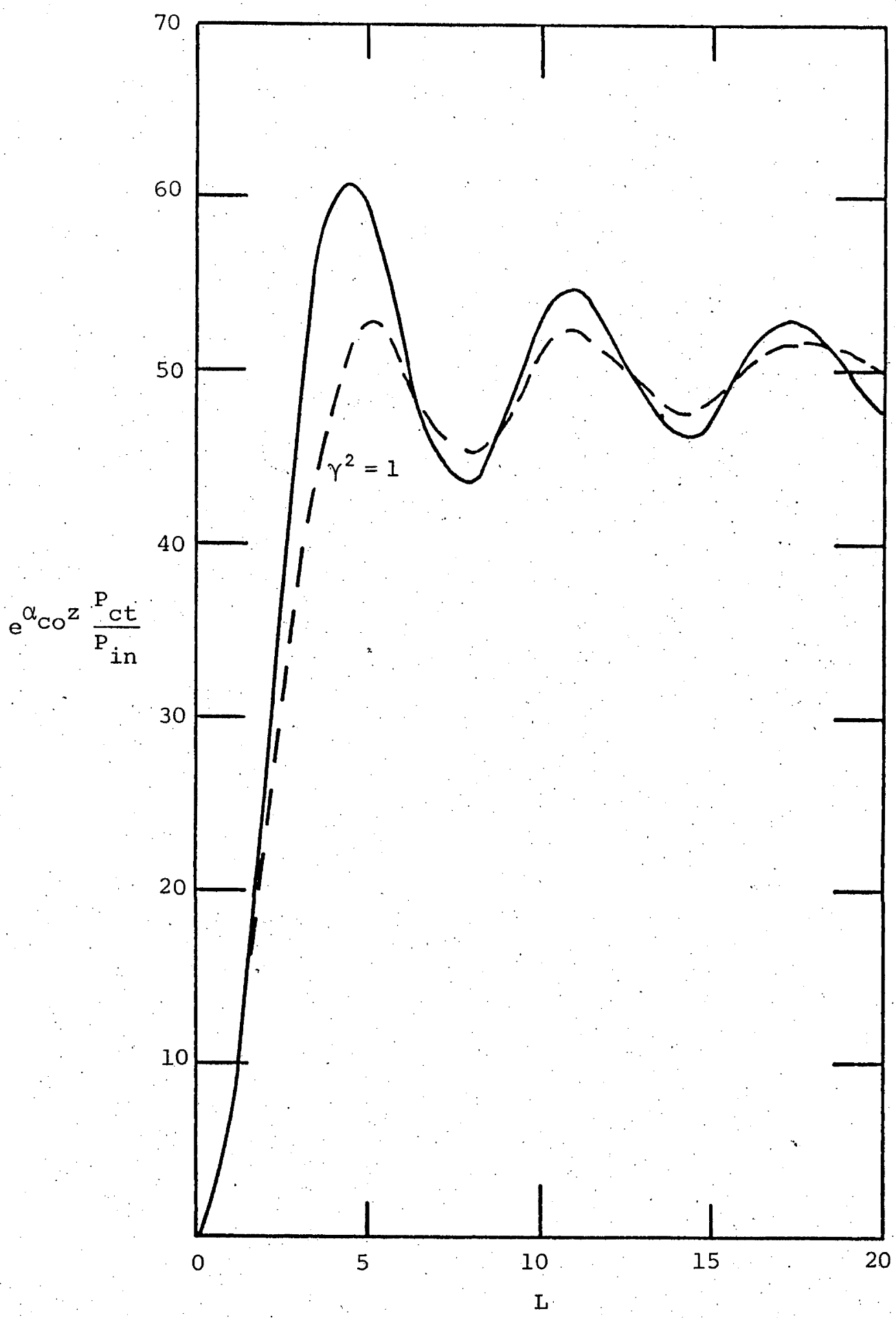

Fig. 5.8. Normalised crosstalk power $\frac{\mathrm{P}_{c t}}{\mathrm{P}_{\text {in }}}$ for the lossless case $\left(\longrightarrow\right.$ and $e^{\alpha_{C O} z} \frac{c t}{\mathrm{P}_{\text {in }}}$ $(-\longrightarrow$ with $\gamma^{2}=\frac{\left(\alpha_{C l}-\alpha_{C O}\right) z}{V}\left(\frac{U_{M}}{V}\right)^{2}=1$. Perfect optics case. 
$\gamma^{2}=1$ which, as stated above, is quite high absorption. Again to a good approximation the crosstalk power is $\frac{P_{c t}}{P_{i n}} e^{-\alpha_{C O} z}$. The very small effect of the cladding loss on crosstalk reflects the fact that most of the modal power is within the fibre when $\mathrm{V}$ is large.

\subsubsection{Unequal-Radius Fibres}

The maximum crosstalk power $F$ is given by

$$
\begin{aligned}
& F=1 /\left(1+X^{2}\right) \\
& X=\frac{1}{2}\left(\frac{\pi D V^{3}}{2}\right)^{\frac{3}{2}}\left(\frac{\Delta \rho}{\rho}\right) e^{V(D-2)}
\end{aligned}
$$

Because of the exponential dependence on $V, D$, and the fact that $V$ is large, the maximum crosstalk between two parallel light pipes is acutely sensitive to small differences in radius. In Fig. 5.9 we show $F$ as a function of difference in radius for $\mathrm{V}=200$; as a comparison we also give $\mathrm{F}$ for $\mathrm{HE}_{11}$ modes with $\mathrm{V}=2.4$. The greater $\mathrm{V}$, the more improbable is significant crosstalk, unless the light pipes are identical. The crosstalk can be calculated from (5.37) or (5.38) by replacing I by $\mathrm{L} / \sqrt{\mathrm{F}}$ and multiplying $\frac{\mathrm{P}_{C t}}{\mathrm{P}_{\text {in }}}$ by $\mathrm{F}$. When $\mathrm{L} \ll \sqrt{\mathrm{F}}$ or $\mathrm{L} \ll \sqrt{\mathrm{F}} \tau^{2}$ respectively these forms are approximately independent of F. The smaller amount of power transferred is balanced by the faster rate of power transfer.

\subsubsection{Hexagonal Array of Fibres}

According to section 5.3.6, the total crosstalk in the surrounding fibres of a hexagonal array of identical fibres (Fig. 5.4) is given by

$$
\frac{P_{\text {Ct }}}{P_{\text {in }}}=\frac{3}{7}\left[1-\frac{\sin \sqrt{7} L}{\sqrt{7} L}\right]
$$

for the perfect optics case, and 


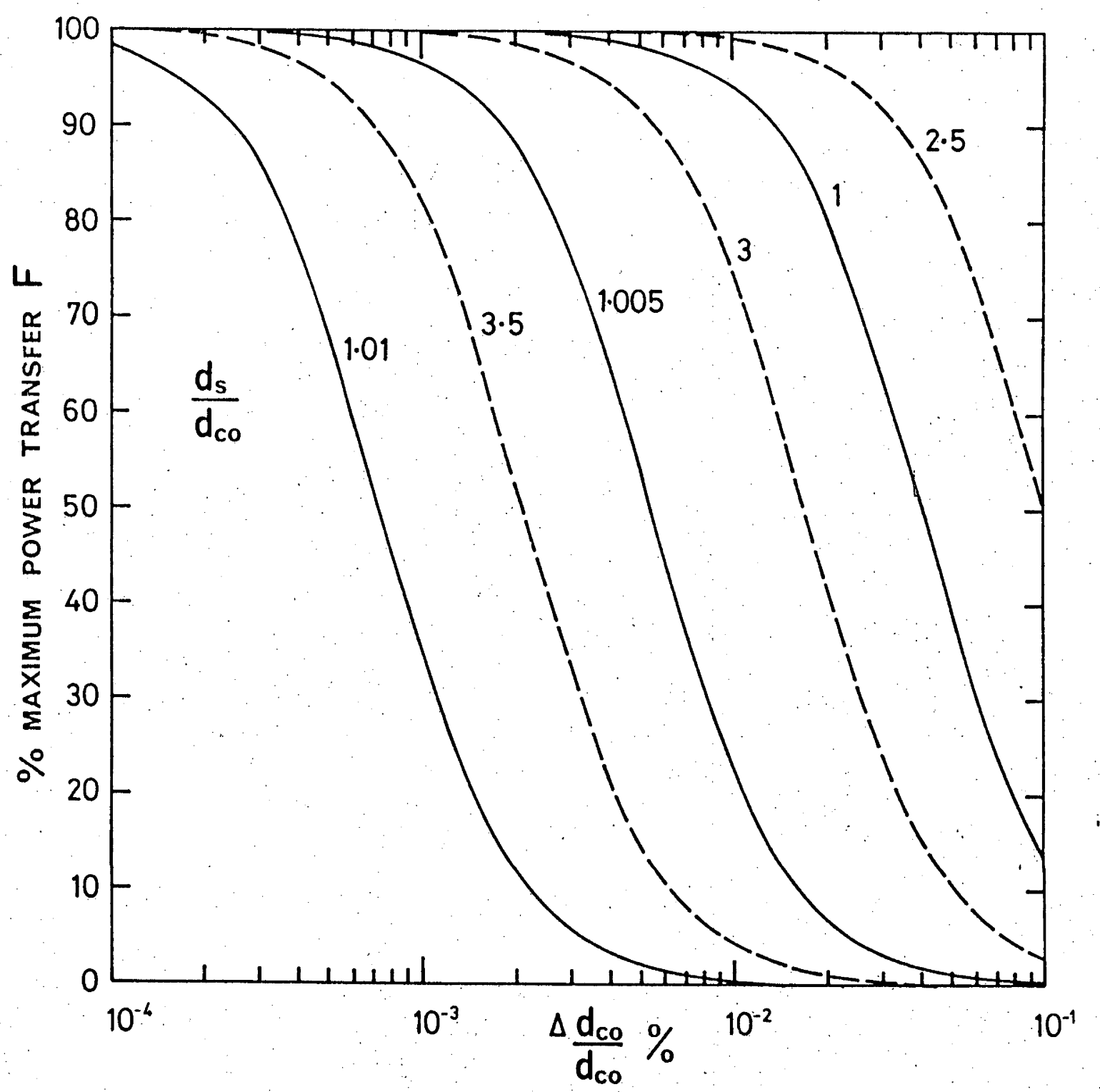

Fig. 5.9 The maximum crosstalk-power transfer $F$ as a function of $\frac{\Delta \mathrm{d}_{\mathrm{CO}}}{\mathrm{d}_{\mathrm{CO}}}=\frac{\Delta \rho}{\rho}$ with $\mathrm{v}=200(\longrightarrow)$. As a comparison, $F$ for $\mathrm{HE}_{11}$ modes with $\mathrm{V}=2.4$ is also given $(--\longrightarrow)$. 


$$
\frac{P_{\text {Ct }}}{\dot{P}_{\text {in }}}=\frac{3}{7}\left[\frac{7 L^{2}}{7 L^{2}+\tau^{4}}\right]
$$

for the imperfect optics case, where all the power is initially in the centre fibre. The crosstalk in each of the surrounding fibres is $1 / 6$ of the total. Fig. 5.10 shows the total crosstalk as a function of $L$ or $\mathrm{L} / \tau^{2}$, together with the corresponding results for two fibres. These curves can be used to calculate the crosstalk in hexagonal arrays of arbitrary dimensions. As we found in Section 3.6, the rate of power transfer is greater in the array, but incomplete power transfer takes place.

\subsection{DISCUSSION}

Two analyses have appeared using ray concepts together with frustrated total reflection, to calculate crosstalk between neighbouring light pipes (Kapany 1959, 1959a, 1967; Cherin and Murphy, 1975). In. addition Kapany $(1959,1959$ a) gives experimental results for an array of touching identical light pipes. Using fibres of $50 \mathrm{\mu}$ diameter, 0.1 inches long and with a refractive index of 1.5 (surrounded by air), he measures a crosstalk ratio $\frac{\mathrm{P}_{\text {out }}}{\mathrm{P}_{\mathrm{Ct}}}=3500$ where $\mathrm{P}_{\text {out }}$ is the output power of the illuminated fibre and $P_{C t}$ is the crosstalk power in one of the surrounding fibres. Unfortunately he does not give all the parameters for the experiment, but if we can take values given in connection with his theory as typical of his experiment, we have $\lambda=0.635 \mu\left(2.5 \times 10^{-5}\right.$. inches) which with $\delta=0.556\left(\theta_{C}=48.2^{\circ}\right)$ gives $\mathrm{V}=276.5$. The lens used to excite the centre fibre has an $f$-number $\left(\cot \theta_{0}\right)$ of 7 giving $\theta_{M}=8.1^{\circ}$ and $U_{M}=34.9$, which falls well within our restriction $U_{M}<V / 3$. A possible source of discrepancy between our mode theory and experiment is a large $\delta$ but as reasonable agreement has been found for $\delta=0.61$ 


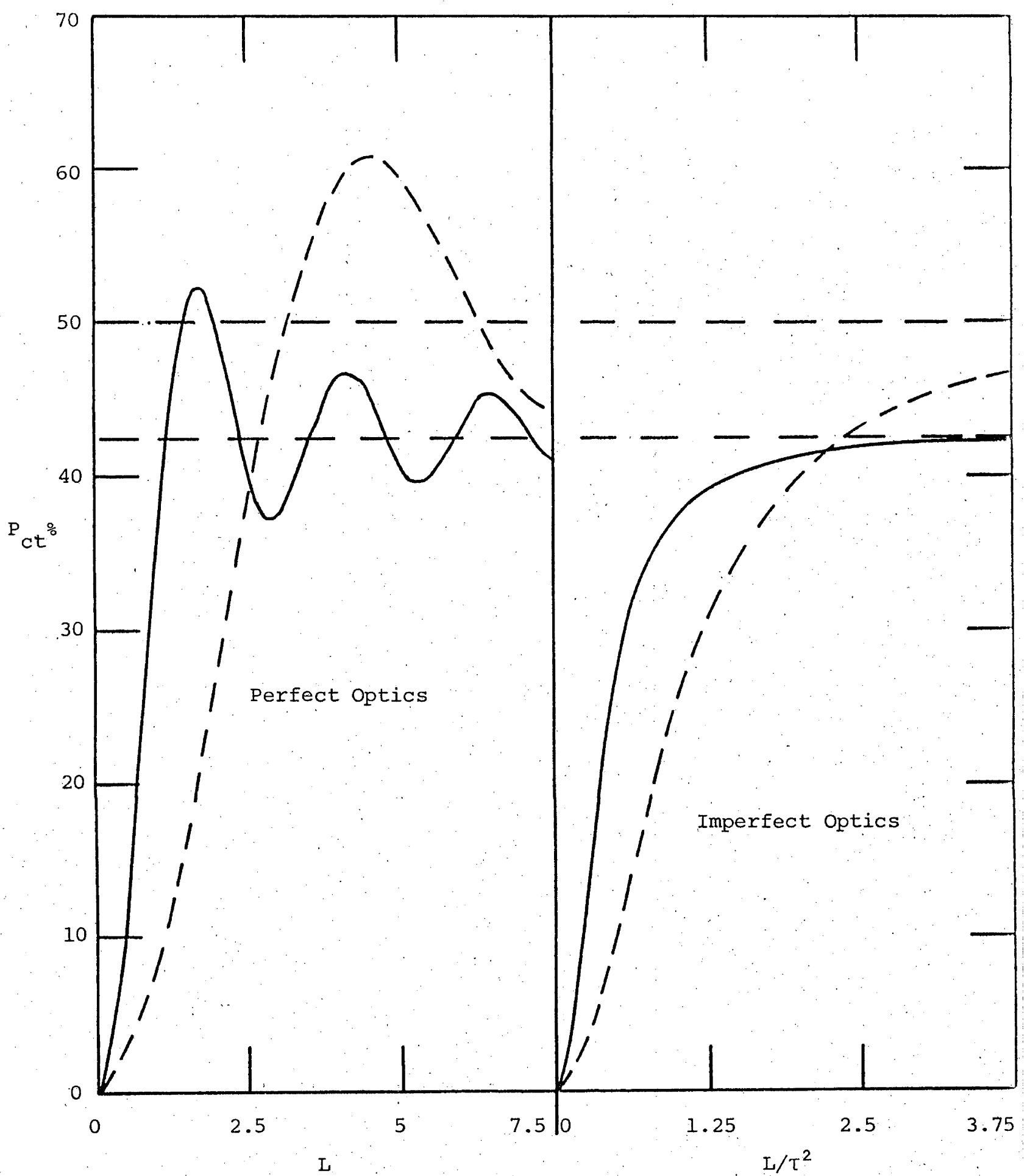

Fig. 5.10. Total crosstalk power $\mathrm{P}_{\mathrm{Ct}}$ in the six surrounding fibres of an hexagonal array of identical fibres, when the central fibre is excited with unity total power $\left(P_{\text {in }}=1\right)(\longrightarrow$. See Fig. 5.4. For comparison the crosstalk power for two fibres is also shown (- - ). 
Snyder, 1972), we would expect the error to be less than $10 \%$ and probably much smaller. Kapany assumes a loss of 1\% per inch but because (1) the total absorption is small, (2) $\theta_{M}$ is small and (3) we calculate here the ratio $\frac{\mathrm{P}_{\mathrm{Ct}}}{\mathrm{P}_{\text {out }}}$, the loss should have negligible effect. $\mathrm{P}_{\text {out }} \approx \mathrm{P}_{\text {in }} \mathrm{e}^{-\alpha_{\mathrm{CO}}{ }^{2}}$ provided the total crosstalk is small. In terms of rays, this requires all rays to have approximately the same path length i.e. $\sec \theta \approx 1$ for $0 \lesssim \theta \lesssim \theta_{M}$ which for $\theta_{M}=8.1^{\circ}$ is a good approximation. From the data we calculate $\mathrm{L}=0.0823$, and using the general expression (5.24) adapted to a hexagonal array $\left(L \rightarrow \sqrt{7} L, \frac{P_{C t}}{P_{\text {in }}} \times \frac{1}{7}\right)$, we we calculate the crosstalk power $P_{c t}$ in one of the surrounding fibres for different degrees of imperfection $\tau^{2}$ as

\begin{tabular}{|c|c|}
\hline$\tau^{2}$ & $\frac{\mathrm{P}_{\text {out }}}{\mathrm{P}_{\text {ct }}}$ \\
\hline 0 & 1776 \\
0.5 & 2023 \\
1.0 & 2329 \\
2.0 & 3165 \\
3.0 & 4385 \\
\hline
\end{tabular}

Obviously the crosstalk power varies considerably with the illumination conditions. In his theory Kapany assumes that all modes (angles) are excited equally (apart from a Fresnel reflection term which is very close to 1 for all the angles considered). This corresponds most closely to $\tau^{2} \approx 1$ (and certainly lies between $\tau^{2}=0,2$ ), so that if Kapany's assumption is correct, we predict approximately 50\% more crosstaik than is actually measured. However as the degree of imperfection increases our results approach the measured value. 
There are several reasons for believing that the experimental values would be less than the predicted values, the most significant being a variation in the physical dimensions of the fibres. A photograph of the fibre array used by Kapany (1959) shows that the fibres do not have sharply defined edges and do not appear to be always in complete contact with each other. This may alter the maximum power transfer F significantly but as we have shown in section 5.4.3, for small $\mathrm{L} / \sqrt{\mathrm{F}}$ the crosstalk power is approximately independent of $\mathrm{F}$. The main effect of a variation in the dimensions of the system is a change in the value of $\mathrm{L}$. In calculating $\mathrm{L}$ for Kapany's array we assumed $D=2$ i.e. touching fibres. If we allow, say, a $0.1 \%$ variation in $D$ the exponential term in $L$ changes from 1 to $\exp (-276.5 \times 0.002)=0.575$, and L from 0.082 to 0.047 , giving

\begin{tabular}{|c|c|}
\hline$\tau^{2}$ & $\frac{\mathrm{P}_{\text {out }}}{\mathrm{P}_{\text {ct }}}$ \\
\hline 0 & 5436 \\
0.5 & 6194 \\
1.0 & 7132 \\
2.0 & 9691 \\
3.0 & 13431 \\
\hline
\end{tabular}

i.e. a factor of 3 decrease in the crosstalk power. Because of this sensitivity to a variation in radius or separation, it seems unreasonable to expect exact agreement between theory and experiment; the theory should instead be used to predict bounds on the crosstalk. More experimental values are needed to show whether the theory is consistent, and an experimental determination of the power distribution due to lens excitation is also desirable. 
Kapany (1959, 1967) and Cherin and Murphy (1975) have used ray concepts together with frustrated total reflection (FTR) (a plane-wave concept) to calculate crosstalk between neighbouring multimode optical fibres when meridional rays are excited in one fibre. Meridional rays in a two-fibre system can be characterised by two angles: the angle $\theta$ that the ray makes with the fibre axis and the angle $\phi$ between the ray path and a line joining the centres of the fibres at any cross-section. Only those rays with small $\phi$, i.e. rays striking the boundary near the line of contact of the fibres, undergo FTR. The rest are totally internally reflected. The FTR transmission and reflection coefficients are found by considering FTR of plane waves at a plane boundary (Born and Wolf, 1970) as a function of the angle of incidence $(\phi)$ and of the thickness of the FTR layer. This is adapted to circular geometry by allowing the thickness also to be a function of $\phi$. By assuming an initial distribution of power with angle, summing over many bounces and integrating over all rays, the crosstalk power can be calculated. Inherent in this quasi-ray theory are several assumptions and approximations which we now outline:

(1) The quasi-ray analysis of two identical fibres takes no account of phase, which can give rise to several sources of error:

(a) the analysis assumes that a meridional ray at angle $\theta$ to the fibre axis couples to both meridional and skew rays also at angle $\theta$ to the axis of the coupled fibre. The meridional ray at $\phi=0$ couples to a meridional ray whereas those rays with $\phi \neq 0$ give skew rays in the coupled fibre. Because the path length (and therefore phase) of a ray depends only on $\theta$, the original meridional ray and the coupled 
meridional and skew rays then remain in constant phase relationship as they travel along the fibre, allowing a simple addition of rays at each bounce. However, the skew rays at $\theta$ do not satisfy the periodicity conditions (i.e. the eigenvalue equation) and are therefore not allowed rays. The eigenvalue equation in the ray limit $V \gg I$ states that only rays with certain angles are permitted, corresponding to the fibre modes. Different modes have different $\theta$ values. Therefore, meridional rays at angle $\theta$ couple to meridional rays also at angle $\theta$, but to skew rays at angles $\underline{\theta}_{i}^{\prime} \neq \theta$. Now the phase relationship between the original ray at $\theta$ and the skew rays at $\theta_{i}^{\prime}$ does not remain constant, so that at each successive bounce interference takes place and the skew rays die out, leaving only the meridional rays. The importance of this consideration is demonstrated by Kapany and Burke (1972) for slab waveguides of different dimensions. They show that unless the phase relationship between the rays in the neighbouring waveguides remains almost constant, little coupling takes place. This is equivalent to our factor $F$ which takes into account phase differences between modes.

Our finding that on identical fibres only like $\mathrm{HE}_{1 \mathrm{~m}}$ modes couple corresponds to meridional rays coupling only to meridional rays. Therefore the ray theory overestimates crosstalk by the amount of power contributed to the crosstalk by the skew rays. This is determined by the FTR transmission coefficient. When the fibres are close together, this function is peaked about the meridional ray because it passes through less of the FTR layer than do the skew rays. When the fibres are further apart, the relative difference in transmission of the meridional and skew rays is not so great and therefore a greater proportion of the coupled power is contained in the skew rays. We would therefore predict that the agreement between the ray and mode theories 
should be better when the fibres are close together than when they are further apart. Comparison of our results with those of Cherin and Murphy (1975) shows that this is indeed the case. There is order of magnitude agreement at small $\mathrm{d}_{\mathrm{s}} / \rho(\sim 2.2)$, but their theory predicts much greater crosstalk than ours as the fibres move apart.

(b) Even when only meridional-ray coupling is allowed $(T(\theta ; \phi) \rightarrow T(\theta, 0) \delta(\phi))$, we must still consider phase. Meridional-ray coupling in like fibres is equivalent to coupling of rays in like slabs, a problem analysed by Kapany and Burke (1972). Calculation of the coupling of rays at one angle $\theta$ with and without phase leads to very different results (this corresponds to adding fields or intensities).

Up until now we have been directly comparing the two methods - the ray or geometric optics method and the mode method. An alternative explanation is offered by an analogy with the spatialtransient and spatial-steady-state regions of an optical fibre in isolation (Snyder and Pask, 1975). The spatial-steady-state region is far from the source and only bound modes (trapped rays) are present, whereas in the spatial transient region, close to the source, leaky modes (or leaky rays) which radiate out of the fibre, are present as well. Because the leaky modes radiate, they are not true modes.

In the system of two multimode fibres, the spatial transient region is that described by the geometric-optics or ray method. As we have seen, in the geometric-optics approach there are many rays, i.e. skew rays, which do not correspond to bound modes. Further along the fibre interference effects have caused these skew rays to die out, leaving only the trapped meridional rays which correspond to the bound modes; this is the spatial-steady-state region described by mode theory. Therefore the two methods describe opposite limits - close to and far 
from the source. Consistent with this is the fact that ray theory does not include cross-feedback and is therefore only valid for short lengths of waveguide, and that the simplified coupled-mode theory is not valid for very short lengths i.e. when $\mathrm{cz} / \sqrt{\mathrm{F}} \ll 1$. It appears that no-one has yet found the general solution linking the geometric optics solution (spatial transient) to the mode-theory solution (spatial steady state). This involves including phase in the geometric-optics method and following rays along the waveguides in order to include cross-feedback. (2) The validity of using the FTR transmission coefficient, calculated using plane parallel boundaries, for curved boundaries has yet to be determined. Corrections to the usual Fresnel coefficients have been found for reflection and refraction at a curved boundary (Snyder and Love; 1975), and similar corrections to the FTR transmission coefficient (and including non-parallel boundaries) may be important when the fibres are not touching, and will need to be included in the full theory when phase is considered.

In practice it is difficult to achieve perfectly uniform fibres. Any variation in the radius of the fibres will cause mode mixing for which the geometric-optics method may also be useful (Arnaud, 1975). 
Having developed a theory for coupling between $\mathrm{HE}{ }_{1 \mathrm{~m}}$ modes on multimode optical fibres excited by a lens, we would like to be able to generalise it to include other possible types of excitation. Obviously any excitation which excites only the circularly symmetric $\mathrm{HE}_{1 \mathrm{~m}}$ modes is easily accounted for, with the only possible difficulty being in calculating the resulting integrals analytically. However problems arise when modes other than $\mathrm{HE}_{1 \mathrm{~m}}$ modes are also excited, owing to the dependence of the coupling coefficient and possibly the excitation coefficients $\mathrm{P}_{i}^{\mathrm{q}}$ on the parameter $l$ as well as on $U$. It is theoretically possible to convert the sum over $l$ to an integral following the method of Pask et al (1975) which they use to calculate the total number of modes. The difficulty is in calculating the integrals because the $\ell$ dependence enters as the order of Bessel functions [see (3.58)]. Unless a simple form for the Bessel function is valid, it may be easier and more accurate to calculate the sums rather than the integral approximation. For example, if the fibre is excited by an incoherent source, all modes above cutoff are excited with approximately equal power (Snyder and Pask, 1973). The crosstalk power is given by ${ }_{\text {all modes }} \sin ^{2}\left(\mathrm{C}_{\mathrm{q}} z\right)$ which we convert to a double integral over $U$ and $\ell$. $q$ The integrals over a finite range of the Bessel-function order are not known so we look to the asymptotic form. We are unable in this case to use just the first term of the asymptotic expansion, as we did for the $\mathrm{HE}_{1 \mathrm{~m}}$ modes, because it is not sufficiently accurate for the higher-order modes (for which $\ell^{2} \sim \dot{V}$ ). In fact it predicts that coupling between the odd modes (Section 3.5) is zero, which an exact calculation shows is not the case. Odd coupling is less than even coupling but except for very large separations (VD $\gg \ell^{2}$ ), contributes significantly to the total crosstalk. Use of the first two terms of the asymptotic expansion may be 
valid for some values of the system parameters but gives at best only a rough approximation to the sum. This remains to be investigated. We conclude that, except for specialised types of excitation, it is difficult and probably not greatly accurate to calculate the integral approximation to the mode summation of the crosstalk power. Numerical calculation of the sum is likely to be as fast and has the advantage of accuracy. 


\section{REFERENCES}

Arnaud, J.A. (1975), "Transverse coupling in fiber optics part IV: Crosstalk", B.S.T.J. 54, 1431.

Born, M. and Wolf, E. (1970), Principles of Optics (Pergamon Press, New York, 2nd ed.).

Cherin, A.H. and Murphy, E.J. (1975), "Quasi-ray analysis of crosstalk between multimode optical fibers", B.S.T.J. 54, 17.

Clarricoats, P.J.B. (1975), "Theory of optical fibre waveguides a review", in Progress in Optics, E. Wolf ed. '(North Holland Publishing Co., Amsterdam).

Goodman, J.W. (1968), Introduction to Fourier Optics (McGraw-Hill, San Francisco).

Jackson, J.D. (1962), Classical Electrodynamics (Wiley, New York).

Kapany, N.S. (1959), "Fiber optics. V. Light leakage due to frustrated total reflection", J. Opt. Soc. Am. 49, 770.

Kapany, N.S. (1959a), "Fiber optics. VI. Image quality and optical insulation", J. Opt. Soc. Am. 49, 779.

Kapany, N.S. (1967), Fiber Optics (Academic Press, New York).

Kapany, N.S. and Burke, J.J. (1972), Optical Waveguides (Academic Press, New York).

Marcuse, D. (1972), Light Transmission Optics (Van Nostrand Press, New York).

Miller, S.E., Marcatili, E.A.J. and Li, T. (1973), "Research toward optical-fiber transmission systems", Proc. I.E.E.E. 61, 1703

Pask, C., Snyder, A.W. and Mitchell, D.J. (1975), "Number of modes on optical waveguides", J. Opt. Soc. Am. 65, 356.

Snyder, A.W. (1969a), "Asymptotic expressions for eigenfunctions and eigenvalues of a dielectric or optical waveguide", I.E.E.E. Trans. Microwave Theory Tech. MTT-17, 1130.

Snyder, A.W. (1969b), "Excitation and scattering of modes on a dielectric or optical fiber", I.E.E.E. Trans. Microwave Theory Tech. MTT-17, 1138.

Snyder, A.W. (1972), "Coupled-mode theory for optical fibers", J. Opt. Soc. Am. 62, 1267. 
Snyder, A.W. (1972a), "Power loss on optical fibers", Proc. I.E.E.E. $\underline{60}, 757$.

Snyder, A.W. (1974), "Leaky-ray theory of optical waveguides of circular cross section", Appl. Phys. 4, 273.

Snyder, A.W. and Love, J.D. (1975), "Reflection at a curved dielectric interface - electromagnetic tunnelling", I.E.E.E. Trans. Microwave Theory Tech. MTT-23, 134.

Snyder, A.W. and Mitchell, D.J. (1974), "Leaky rays ón circular optical fibers", J. Opt. Soc. Am. 64, 599.

Snyder, A.W. and Pask, C. (1973), "Incoherent. illumination of an optical fiber", J. Opt. Soc. Am. 63,..806.

Snyder, A.W. and Pask, C. (1975), "Optical fibre: Spatial transient and steady state", Opt. Comm. 15, 314 .

Snyder, A.W., Pask, C. and Mitchell, D.J. (1973), "Light-acceptance property of an optical fiber", J. Opt. Soc. Am. 63, 59. 
CHAPTER 6

CROSSTALK BETWEEN ABSORBING OPTICAL FIBRES

\subsection{OUTLINE}

Coupled-mode theory is used to analyse crosstalk between lossy optical fibres and to determine the distribution and wavelength dependence of the power absorbed in the fibres. We find that for a weakly lossy fibre in the presence of a neighbouring fibre:

(1) there is an increase in the absorption at shorter wavelengths relative to the absorption at longer wavelengths due to crosstalk;

(2) there is an overall decrease in the absorption compared with a fibre in isolation because of the redistribution of the power by the crosstalk. Owing to the wavelength dependence of coupling, the shorter wavelengths are less affected by crosstalk than are the longer wavelengths, thereby giving rise to the effect in (1);

(3) the effect of varying $V$, the separation and the length of the fibres is determined. A decrease in crosstalk reduces the effects in (1) and (2);

(4) when more than one mode is excited a neighbouring fibre can act as a mode filter, reducing the proportion of power in the higher-order modes of the illuminated fibre.

\subsection{INTRODUCTION}

In the preceding chapters we have been mainly concerned with lossless fibres whereas in practice all fibres are lossy. An exact 
solution of the mode equations including loss is difficult, but fortunately in most cases of interest the loss per unit length is small and very accurate perturbation solutions can be found (Reisinger, 1973). These perturbation solutions have a simple, intuitive physical interpretation (Snyder, 1972a) and can be easily incorporated into a coupled-mode theory (Snyder, 1972; Section 6.3), thereby enabling the problem of crosstalk between neighbouring, weakly absorbing optical fibres to be analysed.

Loss can be regarded in two ways: either as a nuisance to be minimised as in communications fibres, where the quantity of interest is the non-absorbed power, or as an essential process as in vision, where the power absorbed in the photoreceptors gives rise to the visual response (Burkhardt, 1962). In this chapter we will be mainly concerned with the amount of power absorbed and its dependence on the wavelength $\lambda$ and on the fibre parameters of a two-fibre system. In particular we show that the absorption spectrum $\mathrm{P}_{A}(\lambda)$ of an individual fibre is changed by the presence of neighbouring fibres. Power is transferred from one fibre to a neighbouring fibre which then absorbs or filters out some of this power which would otherwise have been absorbed by the first fibre. Because coupling is wavelength dependent, the spectrum of power available, and therefore the absorption spectrum of the fibre, is changed - a lateral filtering effect. This is demonstrated in Section 6.4 where the absorption of fibres in a two-fibre system is compared with that in an isolated fibre. The combined effects of more than one mode are discussed in section 6.5 .

This chapter also serves as a theoretical introduction to Chapter 7 in which the effect of crosstalk in visual photoreceptors is examined. 


\subsection{THEORY}

Consider two identical, parallel, circular optical fibres. radius $\rho$, with centres separated by a distance $d_{s}$ and a bulk loss $\alpha(\lambda)$. When an $\mathrm{HE}_{11}$ mode is excited with unit power at $\mathrm{z}=0$ on fibre 1 , the power at any distance $z$ along the fibres is given by (Snyder, 1972)

$$
\begin{aligned}
& P_{1}(z)=\cos ^{2}(C z) e^{-\gamma z} \\
& P_{2}(z)=\sin ^{2}(C z) e^{-\gamma z}
\end{aligned}
$$

where $C(\lambda)$ is the coupling coefficient between $\mathrm{HE}_{11}$ modes on the fibres (Chapter 3) and $\gamma$ is given by

$$
\gamma(\lambda)=\alpha(\lambda) \eta(\lambda)
$$

$\eta(\lambda)$ is the fraction of the power of a mode that propagates inside the fibre and $\lambda$ is the wavelength in vacuum. If the medium surrounding the fibres is also absorbing with bulk loss $\alpha^{\prime}(\lambda),(6.3)$ becomes (Snyder, 1972a)

$$
\gamma(\lambda)=\alpha(\lambda) \eta(\lambda)+\alpha^{\prime} \cdot(\lambda)[1-n(\lambda)] .
$$

The loss term in $(6.1),(6.2)$ is derived by a perturbation method either from coupled-mode theory (Snyder, 1.972) or directly from basic electromagnetic theory (Snyder, 1972a). The method requires that $\alpha / \beta \approx \frac{\sqrt{\delta} \alpha \rho}{v} \ll 1$, or equivalently that the imaginary part of the refractive index be much smaller than the real part. Physically this means that the absorption over a distance equal to a wavelength is small and that the modal fields are the same as for a lossless fibre, but decay exponentially as $e^{-\frac{\gamma z}{2}}$. Reisinger (1973) reviews the characteristics of modes in lossy waveguides and,by calculating an exact form, shows that the expression used here is valid when 
$\alpha / \beta<10^{-2}$, which is satisfied in both photoreceptors and communications fibres.

The power absorbed in each of the fibres is given by (snyder, 1972a)

$$
\mathrm{P}_{A}(\lambda)=\omega \int_{V_{S}} \varepsilon^{\prime \prime}(\lambda)|\underline{E}(x, y, z)|^{2} d V
$$

where $\omega$ is the circular frequency, $\varepsilon^{\prime \prime}(\lambda)$ is the imaginary part of the dielectric constant of the fibres and $v_{f}$ is the volume of the fibre. $\underline{E}(x, y, z)$ is the electric-field vector of the fibre mode given by

$$
|\underline{E}(x, y, z)|^{2}=P(z)|\underline{e}(x, y)|^{2}
$$

where $\underline{e}(x, y)$ is the modal electric vector (Chapter 3). Using the definition of $\alpha$,

$$
\alpha(\lambda)=\omega(\mu / \varepsilon)^{\frac{1}{2}} \varepsilon^{\prime \prime}(\lambda)
$$

where $\mu$ is the permeability of the medium and $\varepsilon$ is the real part of the dielectric constant of the fibres, together with (6.5), (6.6), we find that the power absorbed in a length $\ell$ of either fibre is given by

$$
P_{A}(l, \lambda)=\alpha(\lambda) \int_{0}^{l} d z\left\{\begin{array}{c}
\cos ^{2} c z \\
\sin ^{2} c z
\end{array}\right\} e^{-\gamma z}\left[\left(\frac{\varepsilon}{\mu}\right)^{\frac{3}{2}} \int_{A_{f}}|\underline{e}(x, y)|^{2} d A\right]
$$

where $A_{f}$ is the cross-sectional area of the fibre and the top (bottom) expression refers to fibre I (2). By definition (Snyder, 1969a), the term in square brackets is $\eta(\lambda)$ so that

$$
\mathrm{P}_{\mathrm{A}}(\ell, \lambda)=\alpha(\lambda) \eta(\lambda) \int_{0}^{\ell} \mathrm{dz}\left\{\begin{array}{l}
\cos ^{2} \mathrm{Cz} \\
\sin ^{2} \mathrm{Cz}
\end{array}\right\} \mathrm{e}^{-\gamma z} .
$$

We have neglected the wavelength dependence of mode excitation in (6.9). 
To account for this, the right-hand side of (6.9) should be multiplied by a factor $\mathrm{P}_{\text {inc }}(\lambda)$ (Snyder, 1969b). However in order to separate excitation and coupling effects we take $\mathrm{P}_{\text {inc }}(\lambda)=1$ in all calculations. Equation (6.9) can easily be generalised to higher-order modes using the $\mathrm{P}_{\ell \mathrm{m}}$ modes of Chapter 3 , to different fibres and arrays of fibres by including the maximum power transfer factor $F$ (modified if the absorption coefficients of the two fibres are different) and to non-parallel or tapered fibres. For non-parallel fibres, $\mathrm{Cz}$ is replaced by the averaged value (Chapter 4 )

$$
\int_{0}^{z} C(\xi) d \xi=\bar{C} z
$$

whereas for tapered fibres $C$ is replaced by $\bar{C}, n z$ in the exponent is replaced by $\int_{0}^{z} \eta(\xi) \mathrm{d} \xi$ (Snyder and Pask, 1973a) and the $n(\lambda)$ outside the integral in (6.9) is replaced by $n(\lambda, z)$ inside the integral.

The integral in (6.9) can be evaluated for parallel, nontapered fibres, giving

$$
\begin{aligned}
& \mathrm{P}_{A}(l, \lambda)=\left(\frac{\alpha \eta}{2 \gamma}\right)\left[1-\mathrm{e}^{-\gamma \ell}\right] \pm x(l, \lambda) \\
& \mathrm{X}(\ell, \lambda)=\left(\frac{\alpha \eta}{2 \gamma}\right)\left\{\frac{1+\mathrm{e}^{-\gamma \ell}\left[\left(\frac{2 \mathrm{C}}{\gamma}\right) \sin (2 \mathrm{Cl})-\cos (2 \mathrm{Cl})\right]}{1+\left(\frac{2 \mathrm{C}}{\gamma}\right)^{2}}\right\}
\end{aligned}
$$

where the $+(-)$ sign refers to fibre $1(2)$. The total power absorbed in both fibres $\mathrm{P}_{\mathrm{T}}$ is equal to the power absorbed by a single fibre in isolation i.e.

$$
P_{T}=\left(\frac{\alpha \eta}{\gamma}\right)\left[1-e^{-\gamma \ell}\right]
$$

If the surrounding medium is lossless, $\gamma=\alpha$ and (6.11), (6.12) reduce to 


$$
\begin{aligned}
& P_{A}(l, \lambda)=\frac{1}{2}\left(1-e^{-\alpha \eta l}\right) \pm x(l, \lambda) \\
& x(l, \lambda)=\frac{1}{2}\left\{\frac{1+e^{-\alpha \eta l}\left[\left(\frac{2 C}{\alpha \eta}\right) \sin (2 C l)-\cos (2 C l)\right]}{1+\left(\frac{2 C}{\alpha \eta}\right)^{2}}\right\}
\end{aligned}
$$

The power absorbed is a function of wavelength through the parameter $v$, which determines both the coupling coefficient and $\eta$, and through the bulk absorption coefficient $\alpha(\lambda)$, which must be specified. As the wavelength is increased $\mathrm{V}$ becomes smaller, the rate of power transfer is increased and $\eta$ is decreased.

The expression we use for the coupling coefficient $C$ is that derived in Chapter 3 which assumes lossless media. If loss is included the coupling coefficient is modified to give approximately

$$
\begin{aligned}
C & \rightarrow C\left\{1+\left[\frac{2 \rho\left(\alpha-\alpha^{\prime}\right)}{v \sqrt{\delta}}\right]^{2}\right\}^{\frac{1}{2}} \\
& \approx C\left\{1+2\left[\frac{\rho\left(\alpha-\alpha^{\prime}\right)}{v \sqrt{\delta}}\right]^{2}\right\} .
\end{aligned}
$$

In most cases, including relatively lossy photoreceptors, the second. term in (6.16) is negligible. In fact the second term is approximately $2\left(\frac{\Delta \alpha}{\beta}\right)^{2}<2(\alpha / \beta)^{2}<1$ by our weak-loss assumption.

\subsection{RESULTS}

We wish to determine the effects of crosstalk in a lossy twofibre system on (1) the amount of power absorbed in each fibre compared with a fibre in isolation and (2) the wavelength dependence of the absorption. Two effects can modify the bulk absorption of a medium in such a system. "One is the effect of confining the medium to a waveguide of small cross-section (waveguide effect) which is a 
consequence of the $\eta(\lambda)$ term in the waveguide absorption coefficient $\gamma$. This gives rise to a relative increase in the absorption at shorter wavelengths and occurs on a fibre in isolation (Snyder and Pask, 1973b). The second effect due to crosstalk (coupling effect), is caused by the presence of a neighbouring fibre and is also wavelength dependent through the parameter $\mathrm{V}$ in the coupling coefficient.

In order to evaluate $(6.14),(6.15)$ we must specify a bulk absorption coefficient $\alpha(\lambda)$. We use the absorption coefficient of the visual pigment rhodopsin $\left(\lambda_{\max }=500 \mathrm{~nm}\right)$ with a secondary maximum in the ultraviolet (uv) region (see Chapter 7). In Fig. 6.1 we show the bulk absorption $\alpha(\lambda)$ together with the normalised absorption curves of a fibre in isolation (waveguide effect only) and of the same fibre in the presence of an identical neighbouring fibre (coupling and waveguide effects). There is an enhancement of the absorption at shorter wavelengths due to the coupling effect, resulting in a higher uv peak and a small shift of both peaks towards the shorter wavelengths. This reinforces the waveguide effect. The length $(\ell / \rho=200)$ and maximum absorption $\left(\alpha_{\max } \ell=1.5\right)$ are typical of Dipteran photoreceptors (Chapter 7).

Figure 6.2 shows the actual percentage of the initial power absorbed by the illuminated and coupled fibres compared with a fibre in isolation. Less power is absorbed at the longer wavelengths in the illuminated fibre than in the fibre in isolation, whereas absorption at the shorter wavelengths is almost the same; the opposite is true for the coupled fibre. The coupled fibre filters out the power at longer wavelengths which couples more readily, therefore leaving relatively more power at the shorter wavelengths in the illuminated fibre. This gives rise to greater relative absorption at these 


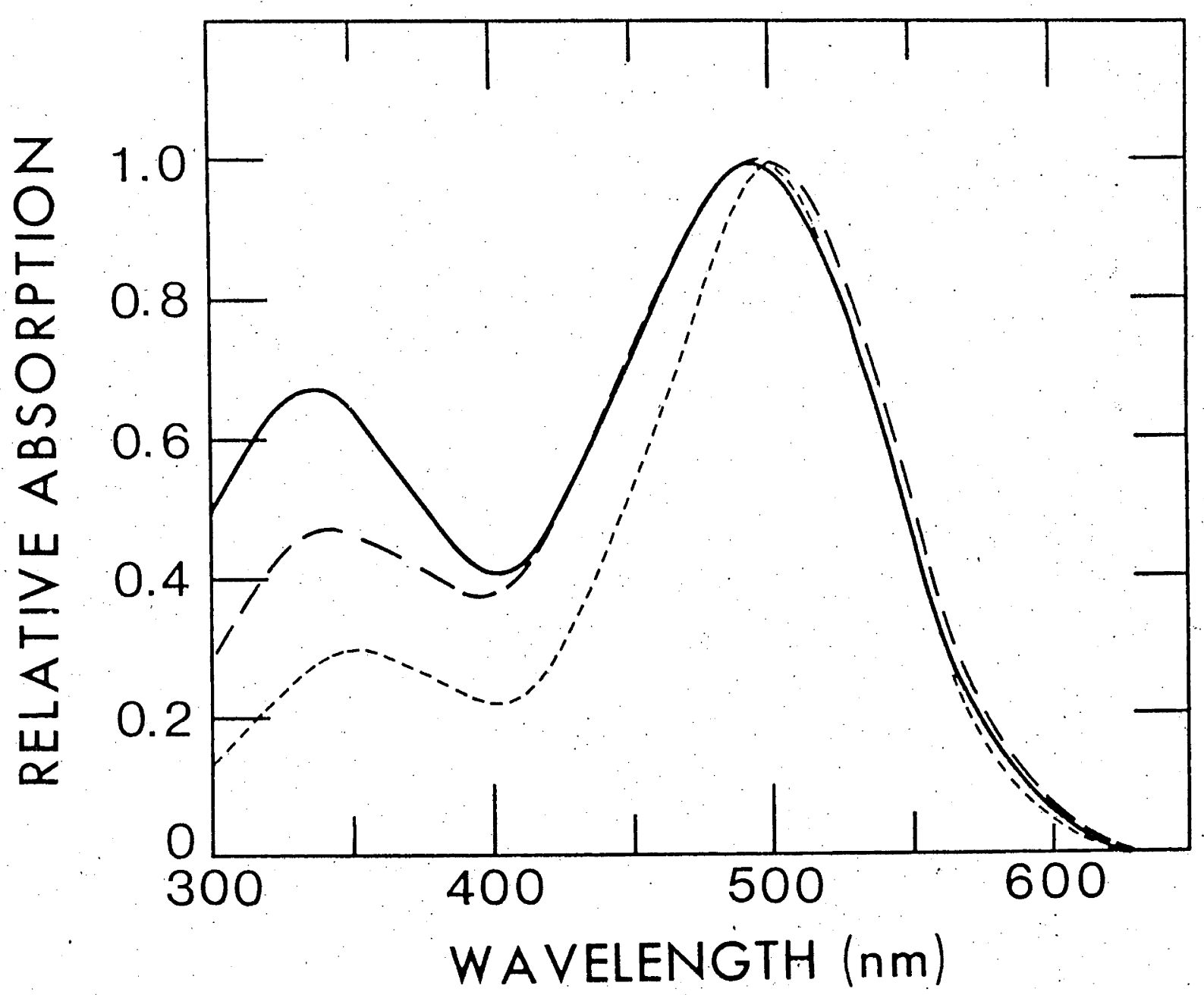

Fig. 6.1. Normalised absorption as a function of wavelength for the illuminated fibre in a two-fibre system (-), for a fibre in isolation ( $--\rightarrow$, and the bulk absorption $\alpha(\lambda)$ used in the calculations (-_-_-_- Comparison of the top two curves shows the enhancement of the ultraviolet peak due to crosstalk between two fibres. $v(\lambda=300 \mathrm{~nm})$ $=4, \delta=0.03, \alpha_{\max } l=1.5, l / \rho=200$ and $\alpha_{s} / \rho=2.2$. 


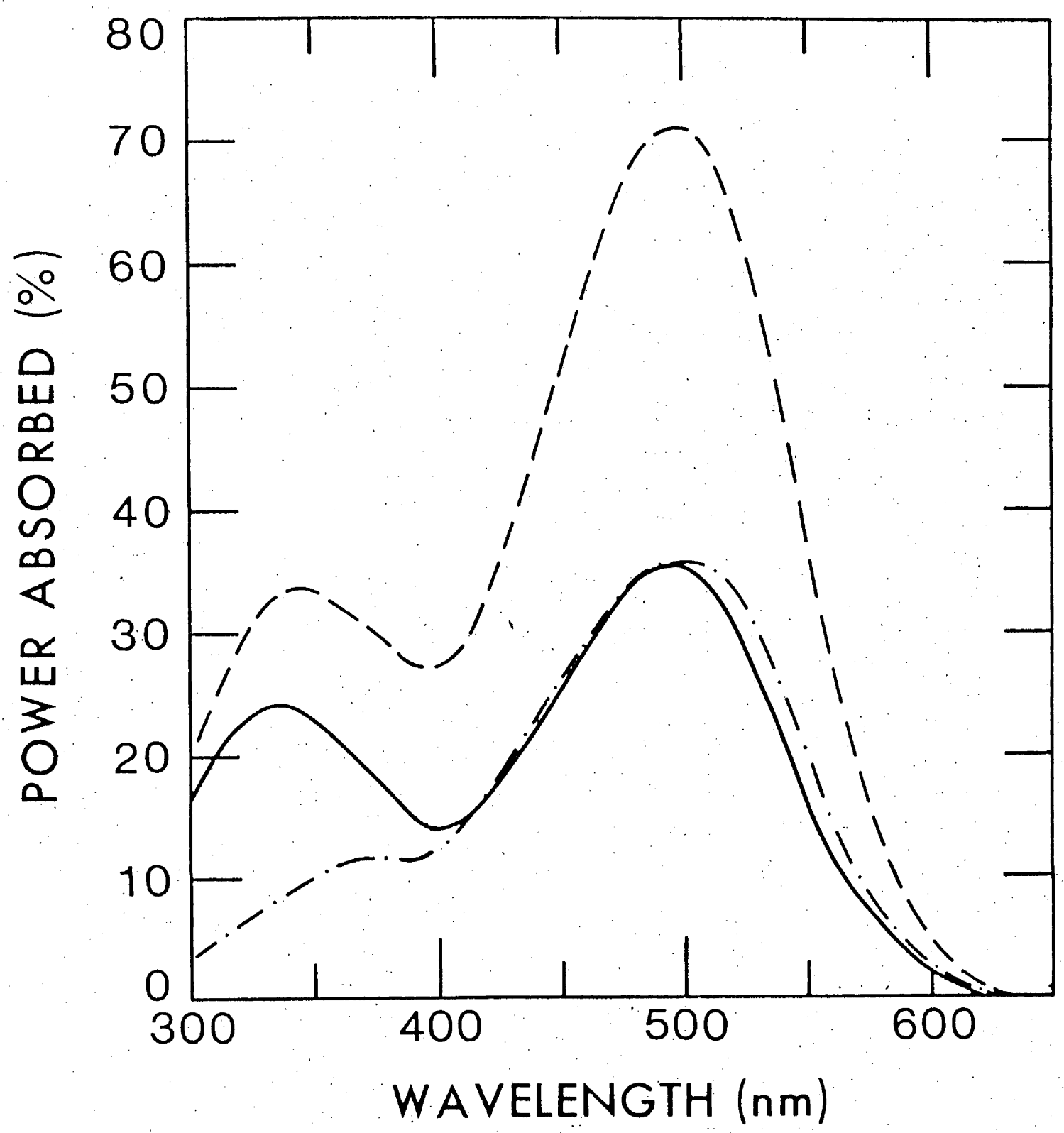

Fig. 6.2. Percentage of the incident power absorbed in the illuminated fibre $(\longrightarrow$ ) and in the coupled fibre (-.-.), compared with a fibre in isolation (- - - ). Parameters are the same as in Fig. 6.1 . 
wavelengths as shown in Fig. 6.1. The relative-absorption curve of the coupled fibre has a flattened uv peak (see Fig. 6.2) because of the preponderance of the longer wavelengths in this fibre.

There is actually a decrease in the absorption in the illuminated fibre at all wavelengths compared with the fibre in isolation because of the distribution of the power between the fibres due to crosstalk. However when the absorption curves are renormalised to a maximum of 1 , we find a greater decrease in the power absorbed at the longer wavelengths; or equivalently a relative increase in the power absorbed at shorter wavelengths.

\section{Effect of $V$ and Fibre Separation}

The difference between the absorption curves of the illuminated fibre in a two-fibre system and of a fibre in isolation depends on the amount of crosstalk, which is a function of $\mathrm{V}$ and of the separation between the two fibres. (There is also a $\mathrm{V}$ dependence in the isolatedfibre absorption curves through the parameter $\eta)$. To quantify this difference we plot in Fig. 6.3 the difference in heights of the uv peaks $(\lambda=340 \mathrm{~nm})$ of the two relative-absorption curves as a function of $\mathrm{V}$ (defined with $\lambda=300 \mathrm{~nm}$ ) for several values of $\mathrm{d}_{\mathrm{s}} / \rho$. This is a measure of the short-wavelength enhancement in the absorption and is also of relevance to photoreceptors (Chapter 7). The difference varies from 0 at large $V$ when very little power transfer takes place, up to a maximum of about 20-30 percent-power-absorbed. As $\mathrm{V}$ is decreased below the region of little coupling, the power at the longer wavelengths, which transfers at a faster rate than that at short wavelengths, begins to couple to the neighbouring fibre where some of it is absorbed. This decreases the power available to the illuminated fibre at the longer 


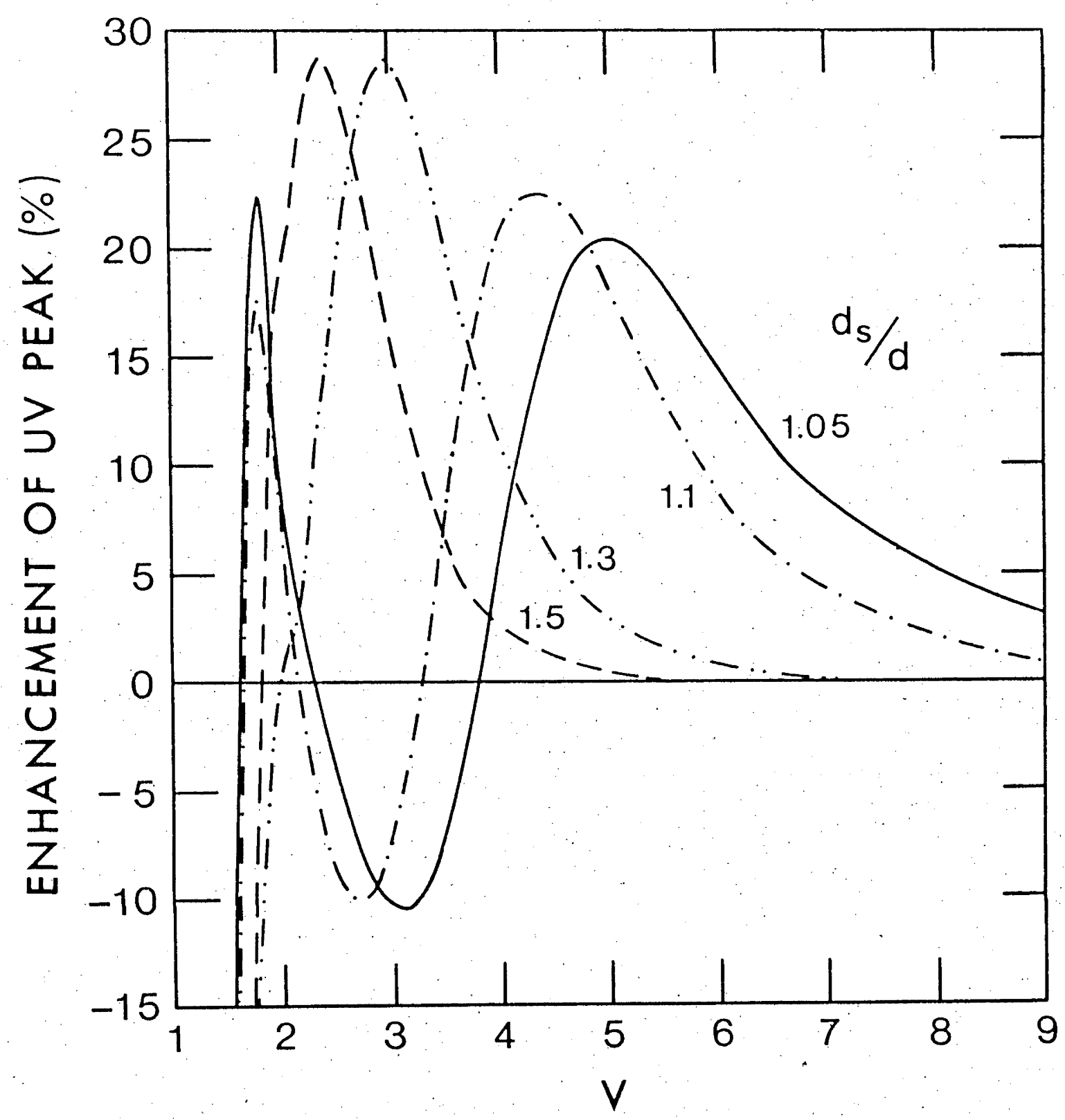

Fig. 6.3. The enhancement of the ultraviolet peak due to crosstalk as a function of $\mathrm{V}$ (defined with $\lambda=300 \mathrm{~nm}$ ). These values are taken from the normalised absorption curves like Fig. 6.1, and represent the difference between the percent power absorbed at $\lambda=340 \mathrm{~nm}$ by the illuminated fibre in a two-fibre system and by an identical fibre in isolation. $\delta=0.03$, $\alpha_{\max } \ell=1.5, \ell / \rho=200$ and $d=2 \rho$. 
wavelengths so that there is a relative increase in the power absorbed at the shorter wavelengths in the iliuminated fibre and therefore an enhanced uv peak. As $\mathrm{V}$ becomes smaller there is greater coupling and the uv enhancement increases until, at the peak value, there is a maximum difference in the effect of coupling at longer $(\lambda \sim 500 \mathrm{~nm})$ and shorter $(\lambda \sim 340 \mathrm{~nm})$ wavelengths. As $V$ is decreased below this peak value the power at shorter wavelengths begins to couple strongly, whereas some of the power at the longer wavelengths is transferred back to the illuminated fibre. Eventually the peak position is reversed; a large amount of power at the shorter wavelengths is coupled to the neighbouring fibre and some of the longer-wavelength power is transferred back to the illuminated fibre before it can be absorbed in the coupled fibre. This gives a negative enhancement and finally a negative minimum of the uv enhancement, corresponding to relatively more power at the longer wavelengths being absorbed in the illuminated fibre. At smaller $\mathrm{V}$ the power transfer begins another cycle and the longer wavelengths transfer once again to the coupled fibre, resulting in a further positive peak.

From Fig. 6.3 it can be seen that fibres further apart give a larger maximum uv enhancement. This is because, up to a certain separation (which depends on the length of the fibres and on $\delta$ ), the further apart the fibres the greater the difference between the coupling of power at the longer and shorter wavelengths. At a given separation, if the fibres are too short, insufficient power transfer will take place over the length of the fibres to reach the peak uv enhancement. Conversely for a given fibre length (and $\delta$ ), there is a value of $d_{s} / \rho$ that gives maximum uv enhancement. 


\section{Effect of length}

The length $l$ of the fibres enters through the exponent

$\gamma \ell$ and through the argument $C l$ of the coupling term. Two identical lossless fibres will completely exchange power over a length determined by the coupling coefficient $C$. The power alternates between the two fibres so that the greater the length $l$, the greater the number of times the power will be exchanged. The same is true for lossy fibres the length for power transfer is approximately the same but, because power is absorbed as it propagates along the fibres, the amount of power transferred is less at each successive exchange.

Increasing the value of $\alpha_{\max } l$ has been shown in the case of a single fibre to flatten the absorption curves giving a straight line i.e. total absorption at all wavelengths, at $\alpha_{\max } l=\infty$, independent of the bulk absorption $\alpha(\lambda)$ (Snyder and Pask, 1973b). This flattening or self-absorption is offset to some extent by the coupling as shown in Fig. 6.4. The absorption curves that include the coupling effect flatten more slowly than the single-fibre curves and at $\alpha_{\max } l=\infty$ the absorption curve still retains some of the shape of the bulk absorption curve.

The distribution of the power absorbed between the two fibres for $\alpha_{\max } l=\infty$ depends on the relative importance of coupling and absorption. If coupling is more important $(2 \mathrm{C} \gg \gamma)$, approximately equal power is absorbed in each of the fibres because the power is exchanged many times between the fibres before it is absorbed. If absorption is more important $(2 \mathrm{C}<\gamma)$, most of the power is absorbed in the illuminated fibre before significant coupling can take place. Because $C$ and $\gamma$ are functions of $\lambda$, both limits can occur on the same absorption curve, giving $100 \%$ absorption in the illuminated fibre at shorter 


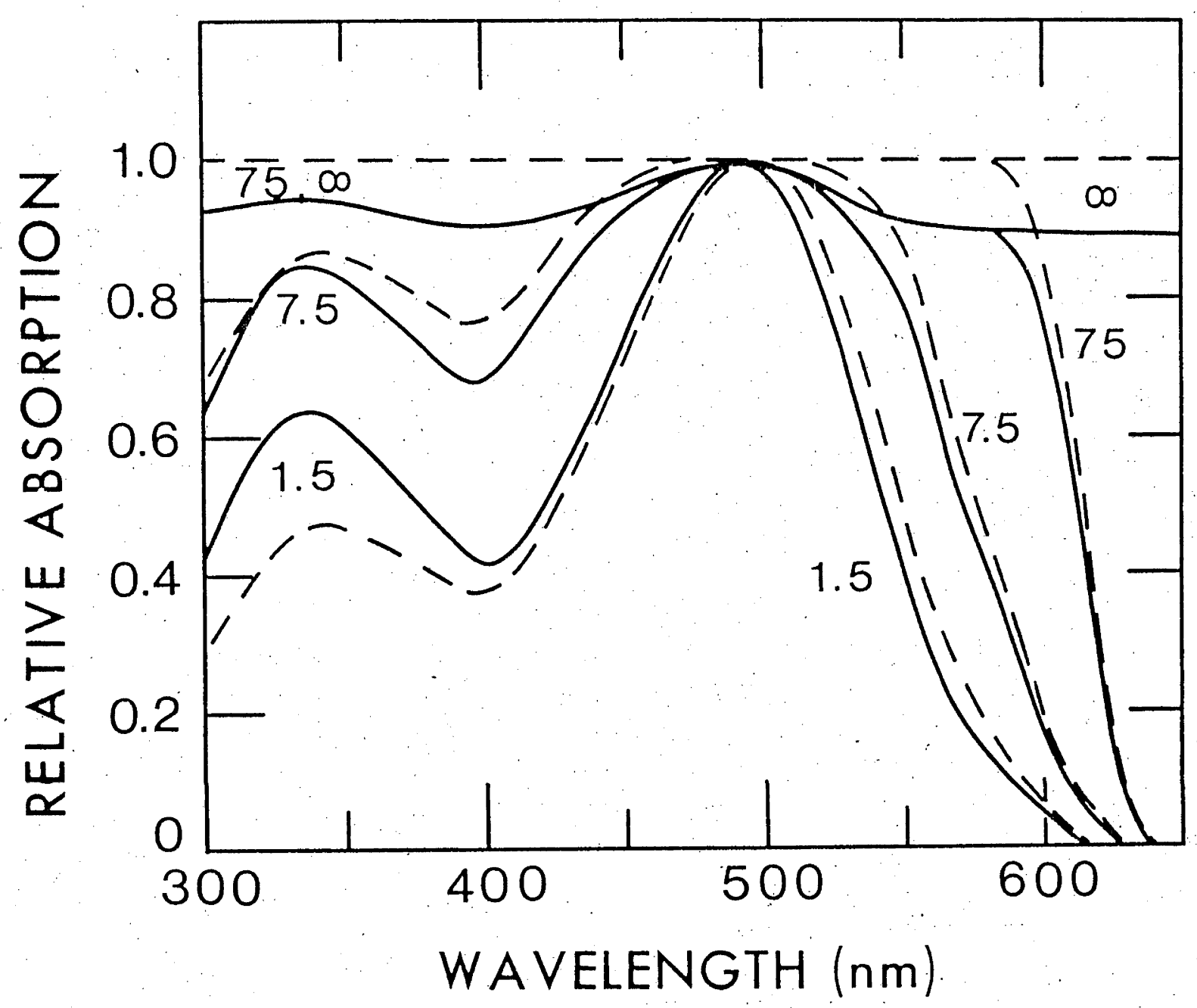

Fig. 6.4. Normalised absorption curves for the illuminated fibre in a two-fibre system ( $\longrightarrow$, and for an identical fibre in isolation (- - - - ), with $\alpha_{\max } l=1.5,7.5,75, \infty$ (corresponding to $\ell / p=200,1000,10000, \infty) . \mathrm{v}(\lambda=300 \mathrm{~nm})=3.5$, $\delta=0.01, \mathrm{~d}_{\mathrm{s}} / \rho=2.2$. 
wavelengths $(2 \mathrm{C}<\gamma)$ and $80 \%$ absorption at the longer wavelengths $(2 C \gg \gamma)$.

\subsection{EXCITATION AND MODE EFFECTS}

In Section 6.3 when calculating the power absorbed we omitted the wavelength-dependent excitation coefficient $P_{\text {inc }}(\lambda)$. In many cases $\mathrm{P}_{\text {inc }}(\lambda)$ is approximately constant (Snyder, 1969b) but it should be included to determine the overall wavelength dependence of the absorption. When more than one mode is excited, the excitation coefficients (as functions of several variables including $\lambda$ ) are needed to determine the initial distribution of power between the modes. In a two-fibre system the presence of more than one mode can give rise to a further coupling effect, owing to the different rates of coupling of the different modes. The higher-order modes couple faster than the lower-order modes so that the neighbouring fibre can act as a mode filter (c.f. wavelength filter in Sections $6.2,6.3$ ) decreasing the proportion of power in the higherorder modes in the illuminated fibre. If the higher-order modes are excited with significant power, the total power absorbed in the illuminated fibre of a two-fibre system can be significantly different to the power absorbed by an isolated fibre with identical excitation conditions. The amount of power absorbed depends on

(1) the excitation conditions, which determine the initial distribution of the power in the modes of the illuminated fibre,

(2) the coupling parameters, which determine the subsequent distribution of power in the modes of the two fibres, and

(3) the absorption parameters, which determine how much of this power is absorbed.

As the power absorbed depends on many parameters we illustrate the 
effect by an example.

As excitation conditions, we consider a uniform unpolarised field incident at angle $\theta$ to the fibre axis and covering only the end of the fibre (Snyder, 1969b). We calculate the power absorbed in the illuminated fibre of a two-fibre system and in an identical fibre in isolation as a function of $\theta$ with the. wavelength $\lambda$ as a parameter. The total power absorbed in the illuminated fibre is given by

$$
\mathrm{P}_{A B S}(\theta ; \lambda)=\operatorname{all}_{\mathrm{q}} \sum_{\text {modes }} \mathrm{P}_{\text {inc }}^{\mathrm{q}}(\theta ; \lambda) \mathrm{P}_{\mathrm{A}}^{\mathrm{q}}(\lambda)
$$

where $\mathrm{P}_{\text {inc }}^{\mathrm{q}}(\theta ; \lambda)$ is the initial power in mode $\mathrm{q}$ (Snyder, 1969b) and $\mathrm{P}_{\mathrm{A}}^{\mathrm{q}}(\lambda)$ is the fraction of the power in mode $q$ that is absorbed in the illuminated fibre, as given by $(6.14),(6.15)$ ( + sign) with the appropriate $\mathrm{C}_{\mathrm{q}}$ and $\eta_{\mathrm{q}}$ inserted. The power absorbed by the fibre in. isolation is given by

$$
\mathrm{P}_{\mathrm{ABS}}(\theta ; \lambda)=\underset{\mathrm{all}}{\sum_{\mathrm{q}}} \mathrm{Podes}_{\text {inc }}^{\mathrm{q}}(\theta ; \lambda)\left\{1-\mathrm{e}^{\left.-\alpha \eta_{\mathrm{q}}{ }^{l}\right\}}\right.
$$

In Fig. 6.5 we plot $\mathrm{P}_{\mathrm{ABS}}(\theta ; \lambda)$ given by $(6.17)$ and $(6.18)$ as a function of $\theta$ for several values of $\lambda$ and normalised to $l$ at normal incidence $\left(\theta=0^{\circ}\right)$. As $\theta$ is increased more of the power is excited in the higherorder modes and therefore relatively less power is available to and absorbed by the illuminated fibre compared with the fibre in isolation, giving a flatter angular-response curve. The fraction of the initial power absorbed by the coupled fibre increases as more power is excited in the higher-order modes.

Because $\mathrm{P}_{\text {inc }}$ is a function of both $\lambda$ and $\theta$, the relativeabsorption curves (like Fig. 6.1) will also change as $\theta$ changes for both the two-fibre system and the fibre in isolation when $P_{\text {inc }}$ is included in the formulation (Snyder and Pask, 1972a). 


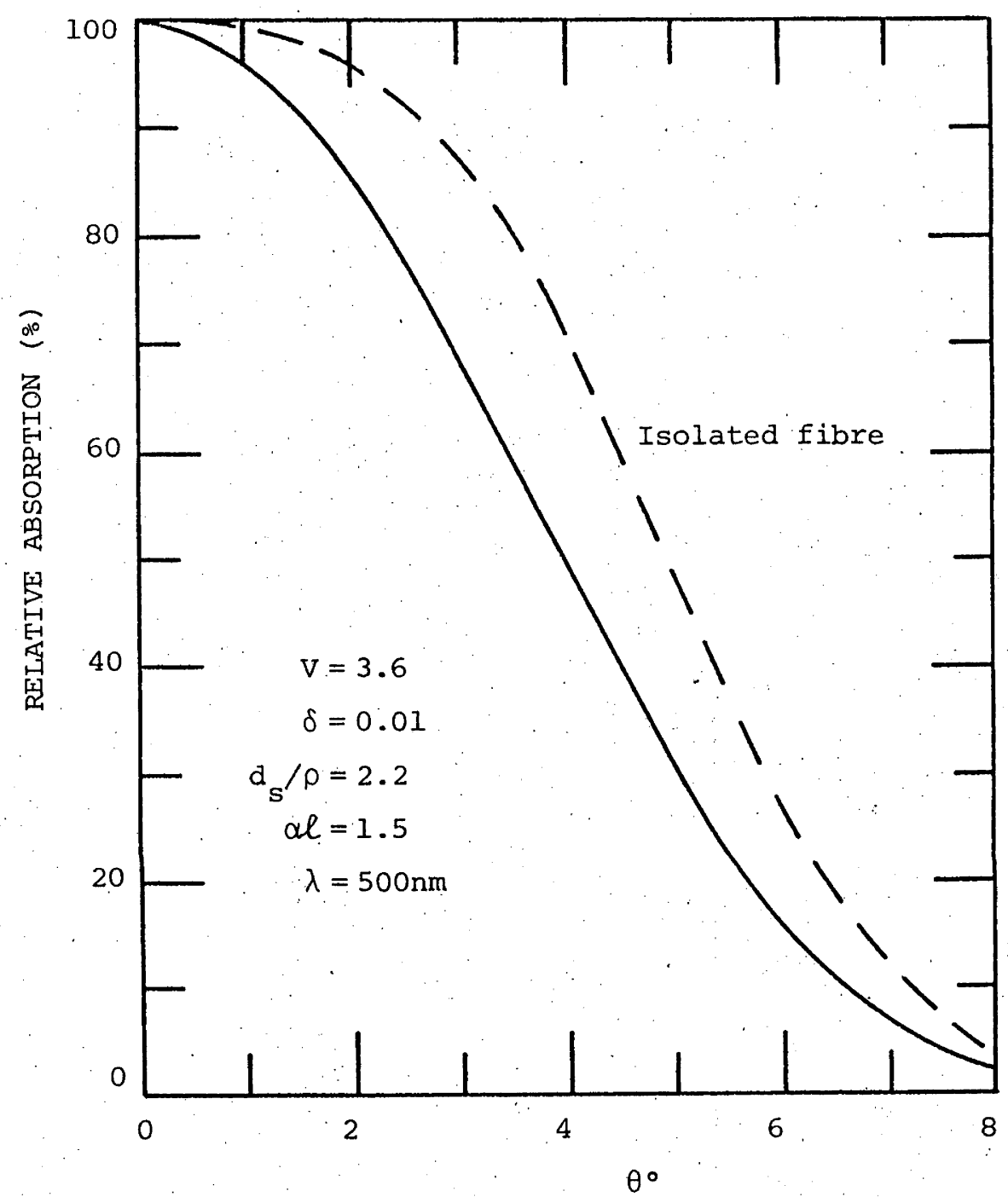

Fig. 6.5. Power absorbed as a function of $\theta$, the angle between the fibre axis and the direction of the incident uniform field, for a fibre in isolation (- $\rightarrow$ and for the illuminated fibre of a two fibre system.

The curves have been normalised to a maximum of $100 \%$ at $\theta=0^{\circ}$. 


\section{REFERENCES}

Burkhardt, D. (1962), "Spectral sensitivity and other response characteristics of single visual cells in the arthropod eye", Symp. Soc. Exp. Biol. 16, 86.

Reisinger, A. (1973), "Characteristics of optical guided modes in lossy waveguides", Appl. Opt. 12, 1015.

Snyder, A.W. (1969a), "Asymptotic expressions for eigenfunctions and eigenvalues of a dielectric or optical waveguide", I.E.E.E. Trans. Microwave Theory Tech. MTT-17, 1130.

Snyder, A.W. (1969b), "Excitation and scattering of modes on a dielectric or optical fiber", I.E.E.E. Trans. Microwave Theory Tech. MTT-17, 1138.

Snyder, A.W. (1972), "Coupled-mode theory for opticàl fibers", J. Opt. Soc. Am. 62, 1267.

Snyder, A.W. (1972a), "Power loss on optical fibers", Proc. I.E.E.E. 60, 757.

Snyder, A.W. and Pask, C. (1972a), "Angular sensitivity of the bee ommatidium", J. Comp. Physiol. 76, 438.

Snyder, A.W. and Pask, C. (1.973a), "Absorption in conical optical fibers", J. Opt. Soc. Am. 63, 761.

Snyder, A.W. and Pask, C. (1973b), "Spectral sensitivity of Dipteran retinula cells", J. Comp. Physiol. 84, 59. 
CHAPTER 7

CROSSTALK BETWEEN VISUAL PHOTORECEPTORS

\subsection{OUTLINE}

The extent of crosstalk between visual photoreceptors, in particular between rhabdomeres in the open rhabdom of flies, is examined in this chapter. We first establish some of the relevant properties of modes on photoreceptors, and then calculate the crosstalk power and the amount of power absorbed in two neighbouring rhabdomeres when only one is illuminated.

We find that:

(I) little power transfer occurs between the peripheral rhabdomeres and the smaller central rhabdomere;

(2) although complete power transfer between two peripheral rhabdomeres can take place, the maximum amount of crosstalk power absorbed by the unilluminated rhabdomere is only about $10 \%$. This may have significance for contrast resolution at low light levels;

(3) the separation of the rhabdomeres increases from the distal end towards the proximal end, except for a slight convergence at the proximal end. This divergence appears to be present specifically to reduce crosstalk;

(4) by tapering, the peripheral rhabdomeres are able to reduce the crosstalk without a reduction in absolute sensitivity. Other reasons for tapering are also examined;

(5) because of the wavelength dependence of crosstalk, a 
neighbouring rhabdomere acts as a lateral wavelength filter and causes an increase in the ultra-violet peak of the spectral sensitivity of the illuminated rhabdomere. The magnitude of the increase is directly proportional to the maximum absorbed crosstalk power. This effect is analogous to optical coupling in fused rhabdoms but its magnitude is much less in the fly.

We conclude that the level of crosstalk between fly rhabdomeres is probably within the limits necessary for their independent operation. Crosstalk does however provide a limitation on the minimum spacing of the rhabdomeres and it appears that, because of crosstalk, flies like Musca have had to suffer a loss of visual acuity in the peripheral rhabdomeres in order to maintain a high absolute sensitivity.

\subsection{INTRODUCTION}

It has been known for nearly a century now that visual photoreceptors have the physical properties of optical waveguides (Brldcke, 1843), but it is only relatively recently that it was realised that waveguide properties could significantly affect the absorption properties of photoreceptors (Toraldo di Francia, 1948). During the last fifteen years or so there has been great interest in photoreceptors as waveguides, in parallel with the development of fibres for optical communications, the two fields having much in common. There have also been improvements and new developments in experimental technique, so that it is now possible for example to measure intra-cellular responses from a single visual cell. As a result of this progress in both theory and experiment it has been shown that the waveguide properties of photoreceptors have a marked influence on their spectral, polarisation, angular and absolute sensitivities (reviews Snyder 1974b, Goldsmith and Bernard, 1974, and 
articles in Snyder and Menzel, 1975).

our aim in this chapter is to examine the effects of a particular waveguide property - crosstalk between individual photoreceptors - on the function of the photoreceptors, and to determine what limitations are imposed by the presence of crosstalk on the design of a visual system. Our studies centre on the retina of certain Dipteran flies. The relevant structure of the rhabdom is outlined in Section 7.3. Crosstalk also plays a role in vertebrate photoreceptors, which are discussed briefly in Section 7.3. In both cases the photoreceptors act as small-V waveguides on which only a few bound modes can propagate. The mode properties of the photoreceptors are discussed in section 7.4 and the mode parameters are established. In order to calculate the crosstalk between what are non-parallel, tapered, absorbing optical fibres, we combine some of the theory of Chapters 3,4 and 6 into a theory section, Section 7.5. Calculated values for the crosstalk power are given in Section 7.6 using data taken from measurements on Musca domestica by Boschek (1971). Because the data vary from species to species and from animal to animal, and because it is not always possible to measure some of the quantities accurately, we also examine the effect on the power absorbed of varying some of the parameters. This also gives some insight into how photoreceptors may have evolved in order to reduce crosstalk. Section 7.7 examines the consequences of crosstalk, the role it plays in determining the structure of a visual system and the effectiveness of the photoreceptors' structure in reducing the crosstalk.

A concurrent study of crosstalk between fly photoreceptors using the theory of Chapters 3,4 and 6 has been presented by Wijngaard and Stavenga (1975), and a general discussion on crosstalk by wijngaard and 
Heyker (1975).

A detailed analysis of the influence of crosstalk in vertebrate photoreceptors is at present underway.

\subsection{STRUCTURE}

Although photoreceptors exhibit an amazing variety of shapes and sizes, most photoreceptors of vertebrates and invertebrates with well-developed visual systems are roughly long narrow cylinders containing the light-absorbing photopigment, with a diameter of the order of a wavelength of light in the visible spectrum, and having a higher refractive index than the surrounding medium. This gives photoreceptors their light-guiding properties. The photoreceptors in a vertebrate retina are the rods and cones; in invertebrates they are the rhabdomeres, which can form fused rhabdoms as in arthropods e.g. ants, bees, or open rhabdoms as in flies. The rhabdom together with its dioptric apparatus which transmits the light to the rhabdomeres, makes up an ommatidium. The detailed structure and function of the different types of photoreceptors are discussed in many reviews, including articles in Reichardt(1969), Fuortes (1972), Dartnall (1972), Wehner (1972), Horridge (1974), by Goldsmith and Bernard (1974) and in Snyder and Menzel (1975). The measurable properties of a photoreceptor as a light absorber are its absolute, spectral, polarisation and angular sensitivities. We assume that the sensitivity of a photoreceptor is proportional to the amount of light absorbed (Burkhardt, 1964; Snyder, 1974b), so that absolute sensitivity is a measure of how much of the light passing along the photoreceptor is absorbed, spectral sensitivity its dependence on wavelength, polarisation sensitivity its dependence on the polarisation 
of the incident light and angular sensitivity its dependence on the angle of incidence of the incident light beam. Experimentally the sensitivity is obtained from receptor potential measurements, using the voltageintensity characteristics of the photoreceptor cell.

We now consider the structure of Dipteran photoreceptors that is relevant to crosstalk.

\section{Dipteran Photoreceptors}

The organisation of the Dipteran visual system has been analysed in meticulous detail (reviews Kirschfeld, 1972; Trujillo-Cenóz, 1972; Braitenberg and Strausfeld, 1973; Stavenga, 1975). There are eight rhabdomeres in each ommatidium; six peripheral rhabdomeres surround a central one consisting of rhabdomere 7 distally and rhabdomere 8 proximally (Fig. 7.I). The neural superposition theory for Dipteran eyes is given in the references above. Of particular relevance to crosstalk is the assumption of this theory that each of the rhabdomeres is optically isolated from its neighbours (Trujillo-Cenóz, 1972; Wijngaard and Stavenga, 1975).

The important parameters in the calculation of crosstalk between rhabdomeres are their diameter, separation, length, absorption coefficient, refractive index and, to some extent, their shape. Although the basic structure of many fly eyes is similar, there is quite a variation in the parameters, particularly in length, which varies from $70 \mu$ for Drosophiza up to about $350 \mu$ for Calliphora (Wijngaard and Stavenga, 1975), and in Musca domestica varies from $100 \mu$ to $200 \mu$ according to eye region (Boschek, 1971). To a lesser extent diameter, separation and possibly even refractive index vary between species (see Trujillo-Cenóz and Bernard, 1972; Snyder and Miller, 1972; Boschek, 1971). Our calculations 


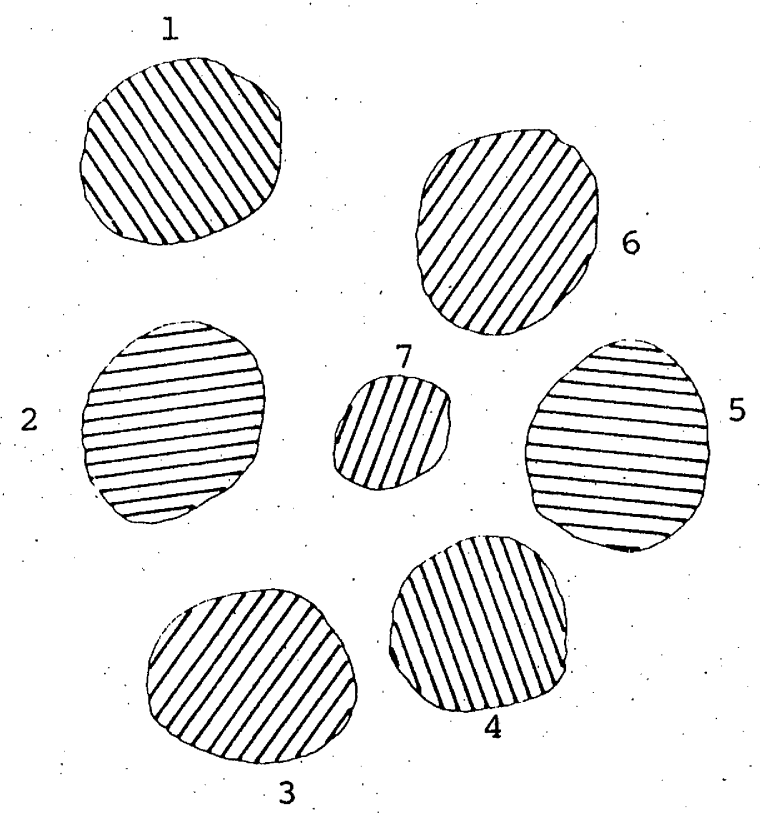

(a)

(b)
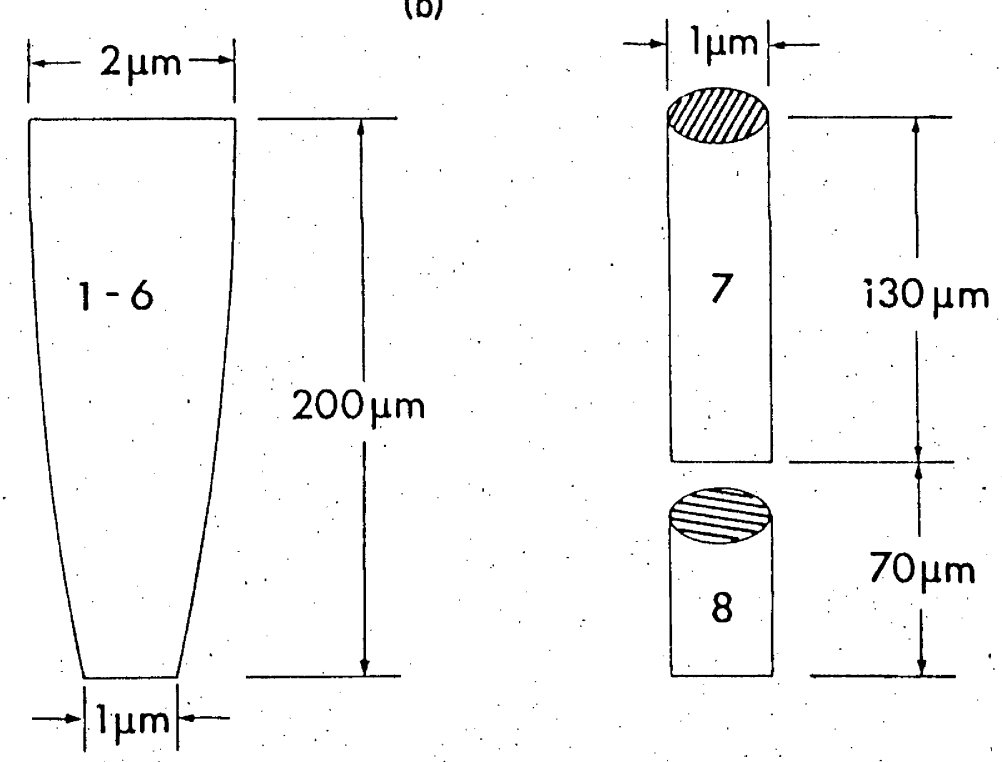

Fig. 7.1. Dipteran rhabdom consisting of six peripheral rhabdomeres surrounding a smaller central rhabdomere made up of rhabdomere 7 distally and rhabdomere 8 proximally. 
in this chapter are based on the measurements of Boschek (1971) on Musca domestica, and also take into account those of Trụjillo-Cenóz and Melamed (1966) and Melamed and Trujillo-Cenóz (1968).

Rhabdomeres 1-6 (Rl-6) of Musca are tapered along their length, the radius decreasing from about $l \mu$ at the distal end to $0.5 \mu$ at the proximal end. Rhabdomeres $7,8(R 7,8)$ have a constant radius of about $0.5 \mu$ (Fig. 7.1). The rhabdomeres are generally elliptical in cross-section, so that we use the equivalent radius measurements of Boschek in our calculations.

The most critical parameter for crosstalk is the separation of the individual rhabdomeres along their length, and it is unfortunate that no systematic investigations of separation have been carried out. For our calculations we use values taken from the serial-section electronmicrographs of Boschek. Several points are worth noting here: Boschek gives photographs for only three depths $(50 \mu, 140 \mu$ and 190 $)$, so that some extrapolation is necessary. In particular the values for the first $50 \mu$ are most important, for it is at this level that the rhabdomeres are closest together. This is reinforced by the finding of Wijngaard and stavenga (1975), who show that after being close together over a relatively short distance, the rhabdomeres appear to separate very rapidly and converge again only at the proximal end. It is not yet certain whether this rapid separation is actually present in the living animal, or whether it occurs as a result of the procedure in preparing the specimen for the electron microscope (Kirschfeld - personal communication); if present in the living animal it has certain consequences for crosstalk which are discussed in Section 7.7.

Another factor in the separation of $\mathrm{Rl}-6$ is the intrusion of the retinular cells for $R 7$ and $R 8$. At the distal end $R I$ and $R 6$ are well- 
separated, because of the intrusion of the retinular cell of R7 (Boschek, 1971 - Fig. 5). More proximally this retinula cell shrinks and RI and R6 converge. At the same depth, the retinula cell for R8 starts to intrude between $\mathrm{RI}$ and $\mathrm{R} 2$, causing these two rhabdomeres to separate. The separation between other pairs of rhabdomeres appears to remain more constant.

The absorption coefficient for fly rhabdomeres 1-6 has been estimated by Kirschfeld (1969) to be $0.005 \mu^{-1}$ for unpolarised light with $\lambda=516 \mathrm{~nm}$ (this is supported by wijngaard and Stavenga, 1975). However this value includes waveguide effects i.e. $\alpha_{\max } n=0.005 \mu^{-1}$. Using the value of $\eta=0.65$ (Section 7.4 ), we obtain $\alpha_{\max } \approx 0.0075 \mu^{-1}$; with length $\ell=200 \mu, \alpha_{\max } \ell=1.5$.

The final parameter of importance for crosstalk is the waveguide parameter $\mathrm{V}$ (see Section 7.4). To calculate $\mathrm{V}$ we need accurate measurements of the refractive indexes of the rhabdomere and the surrounding medium. Seitz (1968) obtained a value of $n_{1}=1.349$ for the refractive index of Calliphora rhabdomeres; this was later corrected for. waveguide effects by Stavenga (1974) to give $n_{1}=1.365$. However doubt exists about the accuracy of the original measurement and there is also difficulty in estimating the refractive index of the surrounding medium, which is non-homogeneous. An alternative, direct method for measuring $\mathrm{V}$ has been found (Kirschfeld and Snyder, 1975), and this is outlined in Section 7.4 .

\section{Vertebrate Photoreceptors}

For the purposes of comparison we give some parameters for vertebrate photoreceptors. In general the outer segments of vertebrate photoreceptors range in radius from about $0.3 \mu$ to greater than $1 \mu$ 


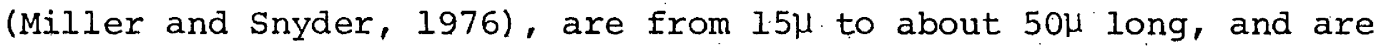
arranged in hexagonal arrays with a centre-to-centre spacing of $1.2 \mu-2.0 \mu$. Values for refractive indexes are given by Miller and snyder (1973) as $n_{1}=1.40, n_{2}=1.34$. An estimate for the absorption coefficient $\left(\alpha_{\max }\right)$ of $0.03 \mu^{-1}$ has been obtained by Liebman and Entine (1968). It is not yet certain whether the inner segments act as waveguides.

\subsection{PROPERTIES OF PHOTORECEPTOR MODES}

Modes have been observed on bleached vertebrate outer segments by Enoch (1963), on worker-bee rhabdoms by Varela and Wiitanen (1970), and on living, in situ outer segments of the rabbit (Enoch, 1967) and rhabdomeres of the fly (Franceschini and Kirschfeld, 1971). This, together with the experiments of Kirschfeld and snyder (1975), demonstrates that photoreceptors are indeed waveguides and that waveguide effects must be taken into account (Snyder, 1974b).

In order to determine the magnitude of the waveguide effects, we must have a good estimate for the parameter

$$
\mathrm{v}=\frac{2 \pi \rho}{\lambda} \mathrm{n}_{1} \sqrt{\delta}
$$

where $\delta=1-\frac{n_{2}^{2}}{n_{1}^{2}}, \rho$ is the radius of the photoreceptor, $n_{1}$ its refractive index, $n_{2}$, the refractive index of the surrounding medium and $\lambda$ the wavelength in vacuum (see Fig. 7.1). As mentioned in the previous section, an accurate determination of $\mathrm{V}$ from refractive-index measurements has proved difficult. Clearly a more direct method for determining $V$ would be advantageous. This has been provided for fly rhabdomeres by Kirschfeld and Snyder (1975) (referred to as KS), who take advantage of the fact that the fraction $\eta$ of modal power that propagates inside the rhabdomere 
(Chapter 3 ) is a function of wavelength and rhabdomere radius through $\mathrm{V}$. Because of this, the measured birefringence is also a function of these two variables (KS; Stavenga, 1975), so that measurement of the birefringence at different wavelengths and with different radii (Rl-6 and $R 7$ ) allows $\mathrm{V}$ to be calculated. In Fig. 7.2 we plot $\mathrm{V}$ as a function of wavelength for fly rhabdomeres RI-6 (distal end) and R7,8 using the data of KS. In their original curve (their Fig. 14), the abscissa should have been wavenumber $(1 / \lambda)$ rather than wavelength, in order to give their straight-line plots. Accordingly their values for $v$ except at $\lambda=300 \mathrm{~nm}$ and $\lambda=656 \mathrm{~nm}$ are incorrect.

The two sets of curves (labelied $A+B, A+C$ ) in Fig. 7.2 are obtained from two different sets of measurements. The $\mathrm{A}+\mathrm{B}$ curves are found by measuring the birefringence of $R 7$ at two different wavelengths to find $v_{7}$, and then multiplying $v_{7}$ by 2 to give $v_{1-6}$ at the distal end where $\rho=1 \mu$. Values of $v_{1-6}$ for other radii of $\mathrm{Rl}-6$, i.e. at different distances along the tapered rhabdomeres, are found from the expression for $V$. The $A+C$ curves are calculated from measurements of the birefringence of $R 7$ and $R l-6$ at the same wavelength, the difference in the measured values being due to the difference in radius. From these data a value for $\mathrm{v}_{1-6}$ is calculated, and by assuming that $\mathrm{R} 7$ has a radius half that of the section of RI-6 used for the measurement, a value of $\mathrm{V}_{7}$ can be found. The important assumption here is that the section of Rl-6 (about $30 \mu-50 \mu$ long) has a radius of about $1 \mu, i . e$. is taken from the distal end. If the section is tapered or if the radius is less than $1 \mu$ (a more proximal section), the values for $\eta_{1-6}$ used in the calculations (KS - Fig. 13) should be smaller; this will lead to a smaller calculated value for $v$. However we find that the measurements are not 


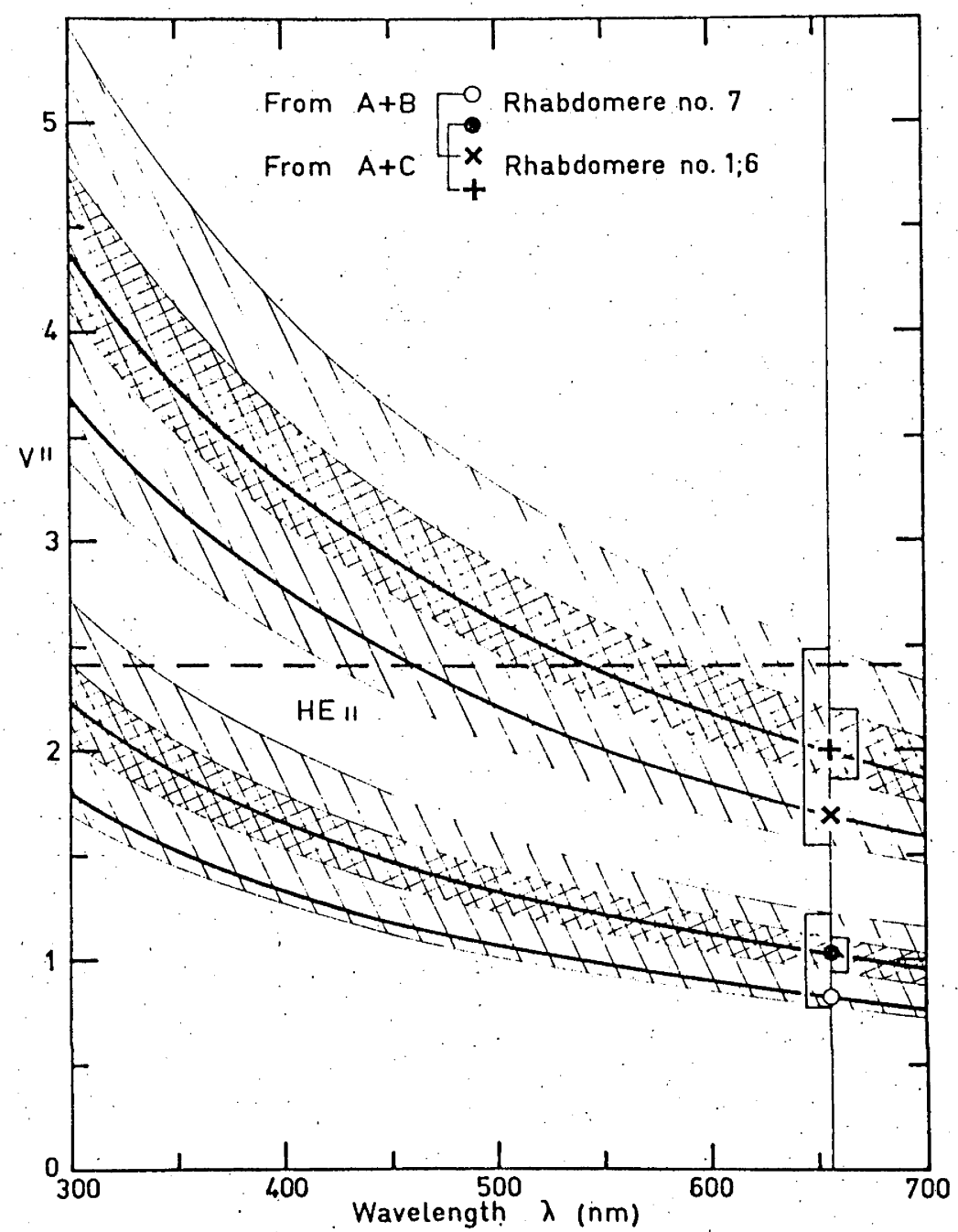

Fig. 7.2. Waveguide parameter $\mathrm{v}^{\prime l}$ for light polarised parallel to the microvilli, as a function of wavelength. The curves are calculated from the measured values of Kirschfeld and Snyder (1975) which are shown by the symbols. The cross-hatching indicates the experimental error. 
very sensitive to a small change in radius, as the following Table shows. $\rho_{\mathrm{av}}$ is the average radius over the rhabdomere length used for the experiment, and is to be interpreted as that radius which gives the correct $\eta$ value for the (tapered) length (Snyder and Pask, 1973a). $\rho_{a v}$ is not the simple geometric average, although there should not be much difference between the two averages. In defining $\mathrm{v}$ for a tapered section, we must associate a $\mathrm{V}$ value with a certain radius, which we take to be $1 \mu$. Given the average radius of the length of rhabdomere, what the experiment actually measures is the quantity $n_{1}^{2}-n_{2}^{2}$; we have calculated $\mathrm{n}_{1}$ in the Table by assuming $\mathrm{n}_{2}=1.339$ (Snyder and Pask, 1973b; Stavenga, 1975). In their calculations, $\mathrm{KS}$ have assumed $\rho_{\mathrm{av}}=1 \mu$.

\begin{tabular}{|l|c|c|c|}
\hline$\rho_{\text {av }}$ & $V(\lambda=656 \mathrm{~nm}, \rho=1 \mu)$ & $V(\lambda=300 \mathrm{~nm}, \rho=1 \mu)$ & $\mathrm{n}_{1}$ \\
\hline 1.05 & 2.03 & 4.44 & 1.356 \\
1.0 & 2.0 & 4.37 & 1.355 \\
0.9 & 1.94 & 4.24 & 1.354 \\
0.8 & 1.85 & 4.05 & 1.353 \\
0.7 & 1.6 & 3.5 & 1.349 \\
0.6 & 1.4 & 3.0 & 1.347 \\
\hline
\end{tabular}

Therefore, provided that the rhabdomere sections are not from the extreme proximal end $\left(\rho_{\mathrm{av}}<0.8\right)$, the results $\mathrm{A}+\mathrm{C}$ in Fig. 7.2 should be valid. The lower curve $(A+B)$ for $v_{1-6}$ gives a value $n_{1}=1.350$, which coincidentally is very close to Seitz' (1968) original measurement. Perhaps two wrongs do make a right! (see KS). Nevertheless we take our values for $v_{1-6}$ from the top curve of Fig. 7.2, which is believed to be the more accurate (Kirschfeld and Snyder - personal communications). Values taken from the lower curve will tend to enhance the waveguide 
effects discussed in this chapter (see Section 7.6). Furthermore these values of $\mathrm{V}$ hold only for dark-adapted eyes; in a light-adapted eye, the pupil mechanism of the fly may alter the value of $\mathrm{n}_{2}$ and so change $\mathrm{V}$ (Kirschfeld and Franceschini, 1969; Stavenga, 1974, 1975; Snyder and Horridge, 1972).

Having arrived at a value for $v$, we are now in a position to discuss some of the properties of modes on photoreceptors. A review of previous work is given by Snyder $(1974 b, 1975)$. An important result from the experiments of $\mathrm{KS}$ is that the central rhabdomere $\mathrm{R} 7,8$ of a fly ommatidium can support only one bound mode (the $\mathrm{HE}_{11}$ or $\mathrm{P}_{01}$ mode) for wavelengths in the ultra-violet and visible range. The peripheral rhabdomeres $\mathrm{R} 1-6$ can however support $a \mathrm{P}_{11}$ mode as well as the $\mathrm{P}_{01}$ mode for $\lambda<550 \mathrm{~nm}$; when $\lambda<350 \mathrm{~nm}$, the $\mathrm{P}_{02}$ and $\mathrm{P}_{21}$ modes may also be present. However if the incident light is on-axis, only the $\mathrm{P}_{\text {om }}$ modes are excited (Snyder, 1969b), so that for $\lambda>350 \mathrm{~nm}$ only one mode; the $\mathrm{P}_{01}$ mode, is excited.

Collectively waveguide effects are, in general, a result of the fact that part of the power of a photoreceptor mode travels along but outside the photoreceptor, and as we have seen in Chapter 3 , this property is all-important for optical crosstalk. Two parameters which characterise this phenomenon are the fraction of the total power which is outside the photoreceptor and the spread of this power out from the photoreceptor boundary. In Fig. 7.3 we plot the fraction of modal power outside the photoreceptor as a function of photoreceptor radius, and for wavelengths covering the uv and visible spectrum. To demonstrate the spread of the power, we show in Fig. 7.4 the fraction of the total power outside the rhabdomere boundary that lies beyond a given radius $r$, i.e. $\frac{\text { Power between the photoreceptor boundary and } r}{\text { Total power outside the photoreceptor }(r \rightarrow \infty)}$, for a photoreceptor 


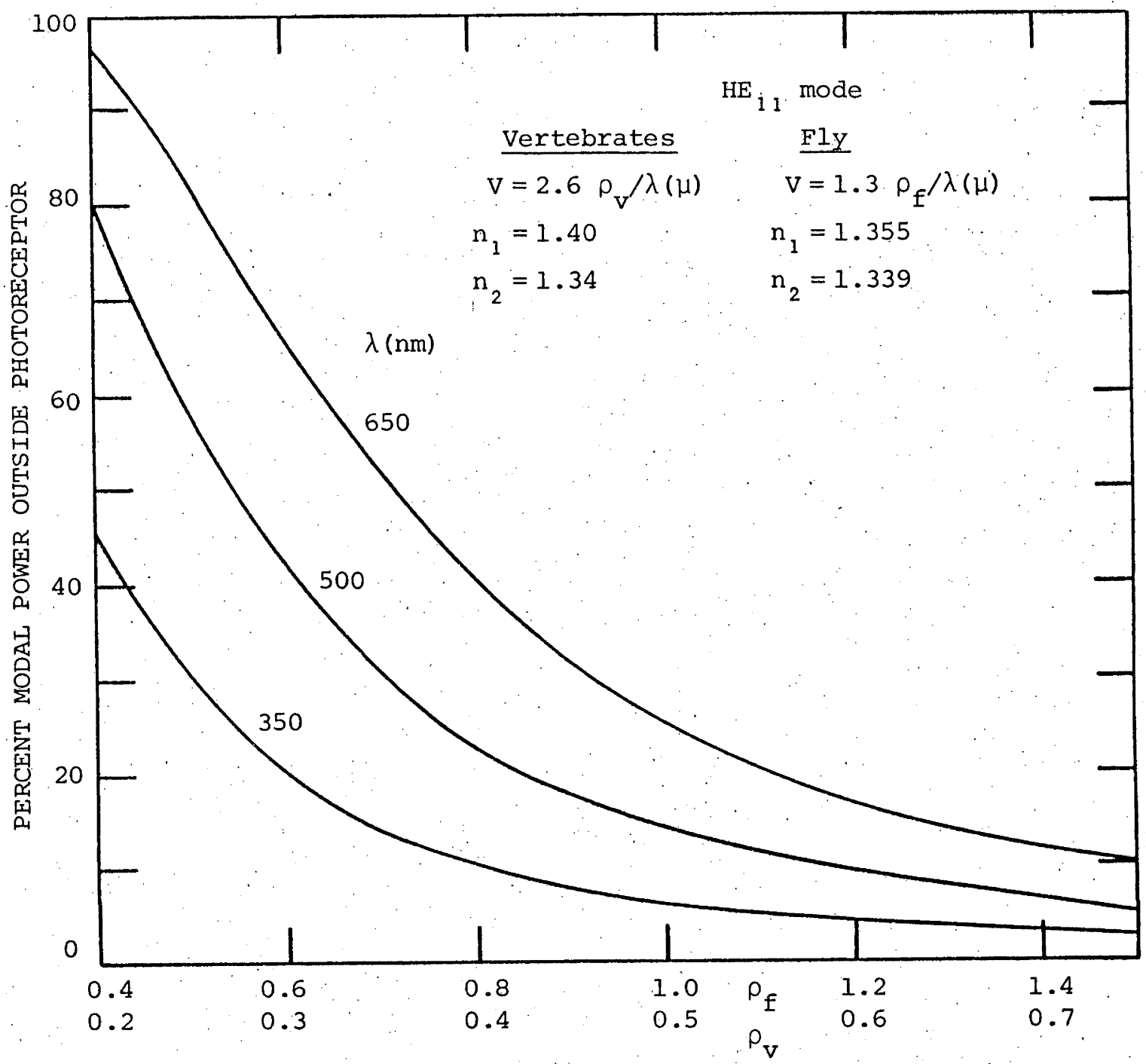

PHOTORECEPTOR RADIUS

Fig. 7.3. Percentage of modal power ( $\mathrm{HE}{ }_{11}$ mode) outside the photoreceptor as a function of radius. The top scale $\rho_{f}$ is for fly rhabdomeres and the bottom scale $\rho_{v}$ for vertebrate outer segments. 


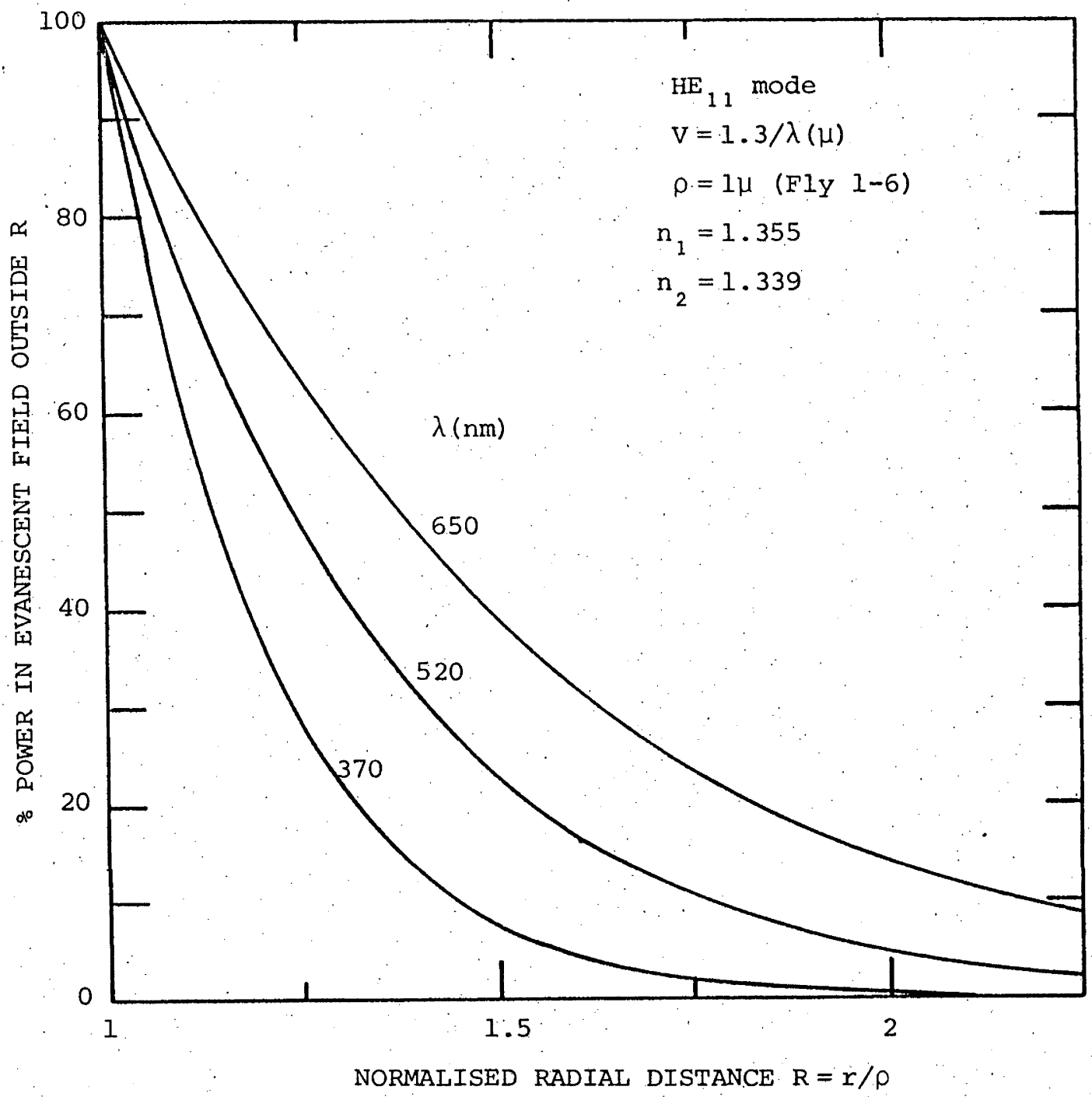

Fig. 7.4. Percentage of the power outside the photoreceptor that lies outside radial distance $r$, as a function of normalised radial distance $r / \rho$. These curves illustrate how the field is spread outside the photoreceptor. 
with the dimensions of the distal part of Rl-6 of fly. Most of the outside power lies within one photoreceptor radius of the photoreceptor boundary in this example. In order to give a general curve for photoreceptors of any radius, we characterise the power spread by a depth of penetration, which is defined to be that distance inside which is contained $90 \%$ of the power outside the photoreceptor, i.e. the value $r_{p}$ of $r$ for which the above ratio is $0.9^{*}$. Therefore only $10 \%$ of the power outside the photoreceptor lies outside $r_{p}$. The normalised depth of penetration $r_{p} / \rho$ is shown in Fig. 7.5 for photoreceptors of different radius. Because the refractive-index values for vertebrate outer segments and fly rhabdomeres are different, we have provided two scales for Figs. 7.3, 7.5. If we can assume that the refractive index of all invertebrate photoreceptors is that of the fly (Stavenga, 1974) and that the values $n_{1}=1.40$, $n_{2}=1.34$ hold for all vertebrate photoreceptors, then Figs. $7.3,7.5$ are valid for all photoreceptors. The figures show that as the radius of the photoreceptor is decreased, the fraction of modal power outside the photoreceptor increases and the power spreads out over a greater distance from the photoreceptor boundary. This gives rise to enhanced waveguide effects (Snyder, 1974b, Section 7.6). The depth of penetration curves can also be used to predict qualitatively how structures outside the photoreceptor e.g. other photoreceptors, screening pigment granules, etc., may affect the absorption of light within the photoreceptor, and consequently the visual response. For example, if there is a significant amount of power outside the photoreceptor and spread over a region which includes neighbouring photoreceptors, we would predict that optical

\footnotetext{
* This definition is different to the usual physics definition of depth of penetration of a field.
} 


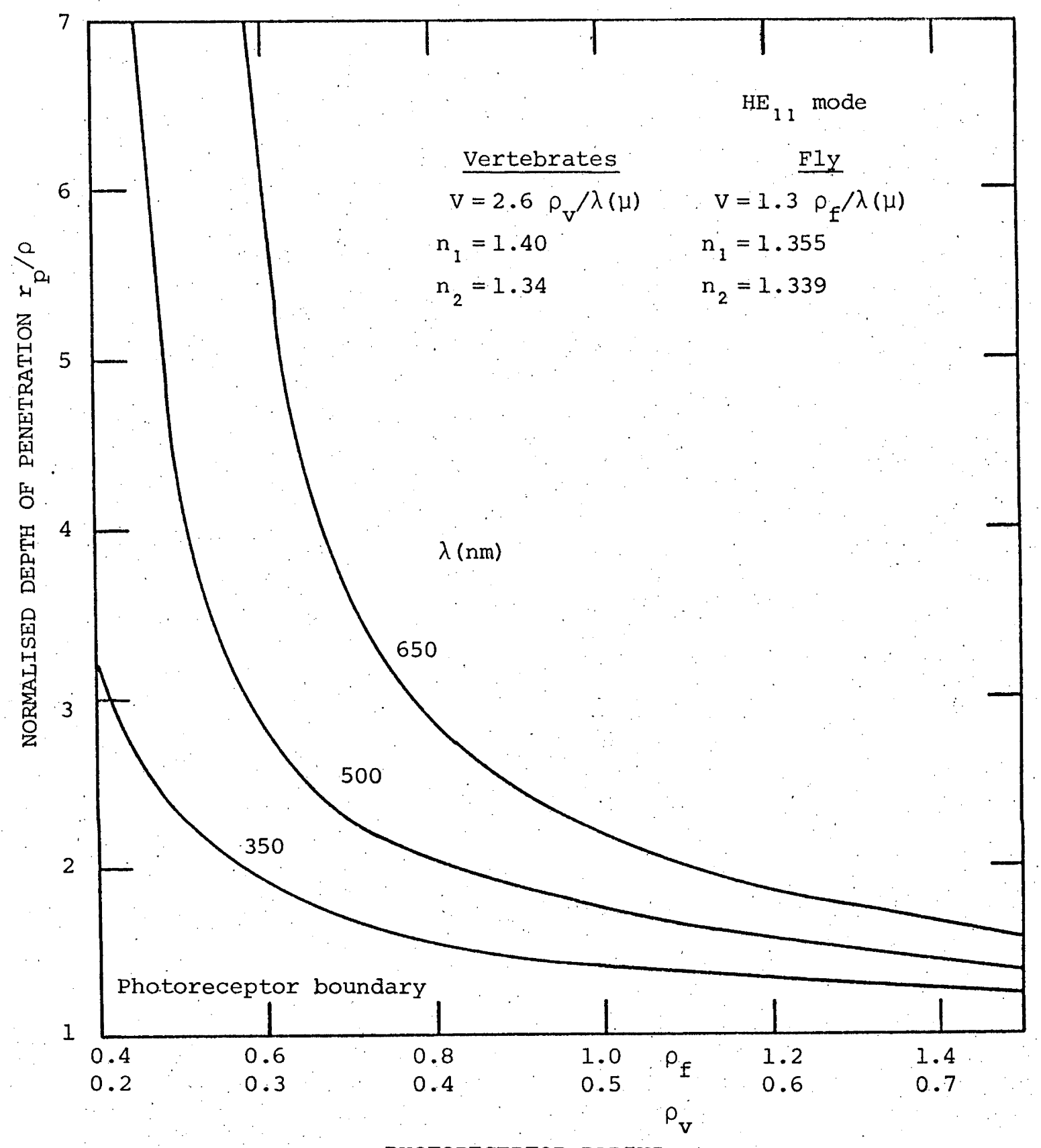

PHOTORECEPTOR RADIUS

Fig. 7.5. Normalised depth of penetration $r_{p} / \rho$ as a function of photoreceptor radius. $90 \%$ of the power outside the photoreceptor boundary lies within $r_{p}$ : As in Fig. 7.3 there are two scales; the top scale $\rho_{f}$ for $f l y$ rhabdomeres and the bottom scale $\rho_{\mathrm{v}}$ for vertebrate outer segments. 
crosstalk might be important. Similarly if there are a number of screening pigment granules within the depth of penetration, they will probably affect the amount of power absorbed by the photoreceptor (Franceschini and Kirschfeld, 1969; Stavenga, 1974, Horridge, 1975).

The role of higher-order modes in vision is as yet uncertain. Higher-order modes have been observed (e.g. Enoch, 1963) and the experiments of KS confirm that they can propagate on fly Rl-6. Several theoretical investigations have shown that these modes do play a part in determining, for example, the angular sensitivity of a photoreceptor (Snyder and Pask,1972b, 1973C, Bernard, 1975; Pask and Snyder, 1975), but in general the effect of higher-order modes is probably small. The fact that most photoreceptors are relatively short means that the degenerate modes of Chapter 3 are valid, but may also introduce problems with the unbound modes (Sammut and Snyder, 1974; Sammut, 1975).

\subsection{THEORY OF CROSSTALK BETWEEN PHOTORECEPTORS}

Using the theory of Chapters 4 and 6 , we calculate the power absorbed in two neighbouring photoreceptors when one is illuminated. We assume non-parallel and tapered identical photoreceptors and that only the dominant $\mathrm{HE}_{11}$ mode is present.

The theory requires a calculation of the averaged coupling constant $\bar{k}$ and the averaged fraction of modal power within the photoreceptor $\bar{\eta}$. The coupling constant for the $\mathrm{HE}_{11}$ mode as a function of $z$ is given by .

$$
K(z)=\frac{U^{2}}{V^{3}} \frac{K_{0}(W D)}{K_{1}^{2}(W)},
$$

where $D=d / \rho$, and in this case $V, U, W$ and $D$ are all functions of $z$. The fraction of modal power within the photoreceptor is given by snyder (1969a) as 


$$
\eta(z)=\left(\frac{U}{V}\right)^{2}\left\{\left(\frac{W}{U}\right)^{2}+\frac{1}{\xi}\right\}
$$

where

$$
\xi=\frac{K_{1}^{2}(W)}{K_{0}^{2}(w)}
$$

In order to transform the expressions for $\bar{\kappa}, \bar{\eta}$ into a more convenient form for integration, we define

$$
\begin{aligned}
& x=\rho_{i} / \rho=v_{i} / v \\
& R=\rho_{i} / \rho_{0}=v_{i} / v_{0} \\
& y=d / d_{s} \\
& s=d_{\ell} / d_{s} \\
& T=d_{s} / \rho_{i}
\end{aligned}
$$

where $d, \rho, v$ are functions of $z$ and $v_{i}=v(0), v_{0}=v(l), \rho_{i}=\rho(0)$, $\rho_{0}=\rho(\ell), d_{s}=d(0), d_{\ell}=d(l)$ are given parameters. In terms of the tapering parameters

$$
\begin{aligned}
z & =\ell\left(\frac{\rho_{i}-\rho}{\rho_{i}-\rho_{0}}\right) \\
& =\ell\left(\frac{1-1 / x}{1-1 / R}\right)
\end{aligned}
$$

In terms of the separation parameters.

$$
\begin{aligned}
z & =\ell\left(\frac{d-d_{s}}{d_{\ell}-d_{s}}\right) \\
& =\ell\left(\frac{y-1}{s-1}\right)
\end{aligned}
$$

Therefore

$$
y=1+(S-1)\left(\frac{z}{l}\right)
$$




$$
=1+(S-1)\left(\frac{1-1 / x}{1-1 / R}\right)
$$

on using (7.10). The normalised separation $D$ is given by

$$
\begin{aligned}
D & =d(z) / \rho(z) \\
& =\frac{d(z)}{d_{s}} \frac{d_{s}}{\rho_{i}} \frac{\rho_{i}}{\rho(z)} \\
& =x y T \\
& =x T\left[1+(S-1)\left(\frac{1-1 / x}{1-1 / R}\right)\right] .
\end{aligned}
$$

If the photoreceptors are parallel, $S=1$ and

$$
\begin{aligned}
D & =x T \\
& =d_{s} / \rho(z)
\end{aligned}
$$

where $d_{s}$ is now constant.

$$
\begin{aligned}
& \text { Given } R, S, T \\
& \begin{aligned}
\bar{K} & =\frac{1}{l} \int_{0}^{l} K(z) d z \\
& =\frac{1}{V_{i}^{3}}\left(\frac{R}{R-1}\right) \int_{1}^{R} \frac{U^{2} K_{0}(W D)}{K_{1}^{2}(W)} x^{2} d x,
\end{aligned}
\end{aligned}
$$

where $\bar{K}$ is defined as $\frac{-\rho_{i} \bar{C}}{\sqrt{\delta}}$ (Chapter 4$), D(x)$ is given by (7.17) and $\mathrm{U}, \mathrm{W}$ are found from the eigenvalue equation (3.23) and (3.22) with $\mathrm{v}=\mathrm{v}_{\mathrm{i}} / \mathrm{x} \cdot \overline{\mathrm{n}}=\frac{1}{l} \int_{0}^{l} \eta(\mathrm{z}) \mathrm{dz}$ is found in a similar manner. From Chapter 6, the power absorbed in the photoreceptors is

given by

$$
P_{A}(l, \lambda)=\alpha(\lambda) \int_{0}^{l} d z n(\lambda, z)\left\{\begin{array}{l}
\cos ^{2}\left(\sqrt{\delta} \bar{k}(z) z / \rho_{i}\right) \\
\sin ^{2}\left(\sqrt{\delta} \bar{k}(z) z / \rho_{i}\right)
\end{array}\right\} e^{-\gamma(z) z}
$$


where the top (bottom) expression is for the illuminated (coupled) photoreceptor, and

$$
\gamma(z)=\alpha(\lambda) \bar{n}(\lambda, z)+\alpha^{\prime}(\lambda)[1-\bar{n}(\lambda, z)] .
$$

$\alpha(\lambda), \alpha^{\prime}(\lambda)$ are the absorption coefficients of the photoreceptor and the surrounding medium respectively and $\bar{k}(z), \dot{\bar{n}}(\lambda, z)$ are the averaged quantities for a length $z$. The integral (7.20) is calculated numerically. The power in the modes on the receptors at any distance $z$ is given by

$$
P(z)=\left\{\begin{array}{l}
\cos ^{2}\left(\sqrt{\delta} \bar{k}(z) z / \rho_{i}\right) \\
\sin ^{2}\left(\sqrt{\delta} \bar{k}(z) z / \rho_{i}\right)
\end{array}\right\} e^{-\gamma(z) z} .
$$

The power actually inside the receptors is obtained by multiplying the expressions in $(7.22)$ by $\eta(\lambda, z)$.

\subsection{RESULTS}

In this section we give quantitative results for the crosstalk between fly rhabdomeres. We assume that initially a $\mathrm{P}_{01}$ mode is excited. with unit power on one rhabdomere $\left(R_{A}\right)$, and calculate the power absorbed in $R_{A}$ and in a neighbouring rhabdomere $R_{B}$ as a function of wavelength. The curves for the power absorbed in $R_{A}$ are also renormalised to a maximum of $100 \%$ to give spectral-sensitivity curves. The explanation for the shape of the spectral-sensitivity curves for fly rhabdomeres, particularly the high uv peak (see Dörrscheidt-Käfer, 1972), is still uncertain (Snyder and Pask, 1973b; Stavenga, 1974; Kirschfeld and Snyder, 1975). Figure 7.6 shows the power absorbed by $R_{A}$ and $R_{B}$ ' together with the power absorbed by $R_{A}$ in isolation (no crosstalk). The values of $V$ 


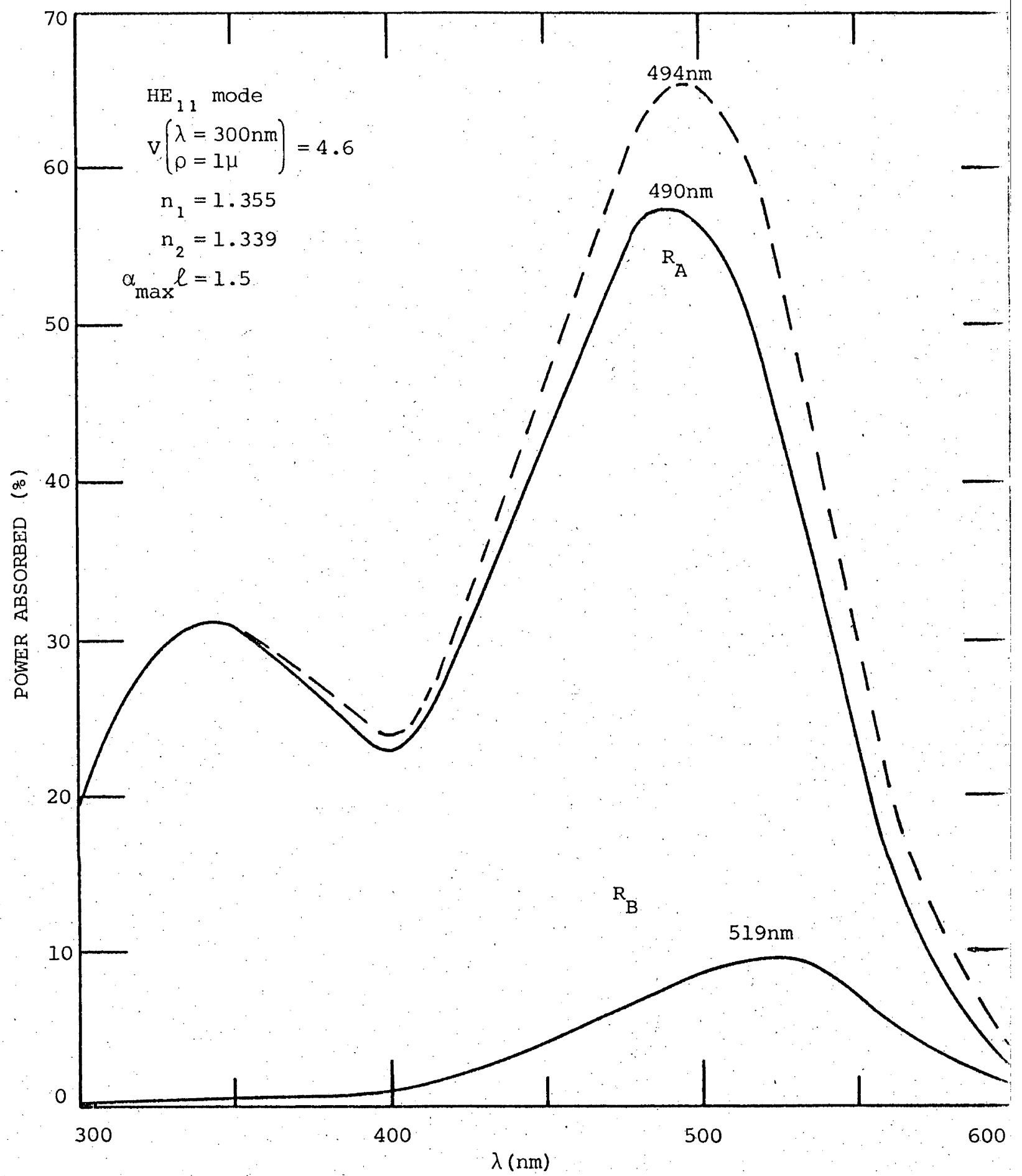

Fig. 7.6. Percent power absorbed by a rhabdomere in isolation (- $-\longrightarrow$, and by a pair of coupled identical rhabdomeres when only one $\left(R_{A}\right)$ is illuminated, as a function of wavelength.

The rhabdomere dimensions are taken from the measurements of Boschek (1971): $\rho_{i}=1.05 \mu, \rho_{0}=0.5 \mu$, initial separation $2.1 \mu$, separation at the distal end $3.6 \mu$, length $200 \mu$. The value for the waveguide parameter $\mathrm{V}$ is taken from Fig. 7.2 . 
are those of Kirschfeld and Snyder (1975) (Section 7.4) and the other data is from Boschek (1971). The values for the separation are taken from Fig. 5 of that paper (see the caption to Fig. 7.6). The spectral sensitivity of $R_{A}$ with and without crosstalk is shown in Fig. 7.7, using the same parameters as Fig. 7.6. For all calculations we use the hypothetical pigment absorption $\alpha(\lambda)$, postulated by snyder and Pask (1973b) and also shown in Fig. 7.7. The difference between the pigment absorption and the spectral sensitivity of a rhabdomere in isolation is due to other waveguide effects (Snyder and Pask, 1973b; Snyder, 1974b), whereas the difference between the two spectral-sensitivity curves is due to the wavelength dependence of crosstalk (Chapter 6). Figure 7.6 shows a maximum absorption of about $10 \%$ in the unilluminated neighbouring rhabdomere $R_{B}$, and Fig. 7.7 a consequent enhancement of the uv peak of the spectral sensitivity of $\mathrm{R}_{\mathrm{A}}$ of about $6 \%$.

The crucial parameter in the crosstalk calculations is the separation of the rhabdomeres. The measurements taken from Boschek's photographs are at best approximate, and in the light of the findings of Wijngaard and stavenga (1975), may be a wrong interpretation of the actual situation. In order to cover the possible range of separations we plot in Fig. 7.8 the maximum power absorbed by $R_{A}$ and $R_{B}$, and the enhancement of the uv peak of $R_{A}$, as a function of normalised separation $d / \rho_{i}$. Although these values are calculated for paraliel tapered rhabdomeres, the curves may be used to predict the effect of varying the separation (in percentage terms) between non-parallel tapered rhabdomeres. Detailed experimental measurements of rhabdomere separation are necessary for a more exact calculation of the crosstalk.

The other parameters whose accuracy is in some doubt are $\mathrm{V}$ (or $\mathrm{n}_{1}$ ) and the maximum absorption. In Fig. 7.9 we plot 


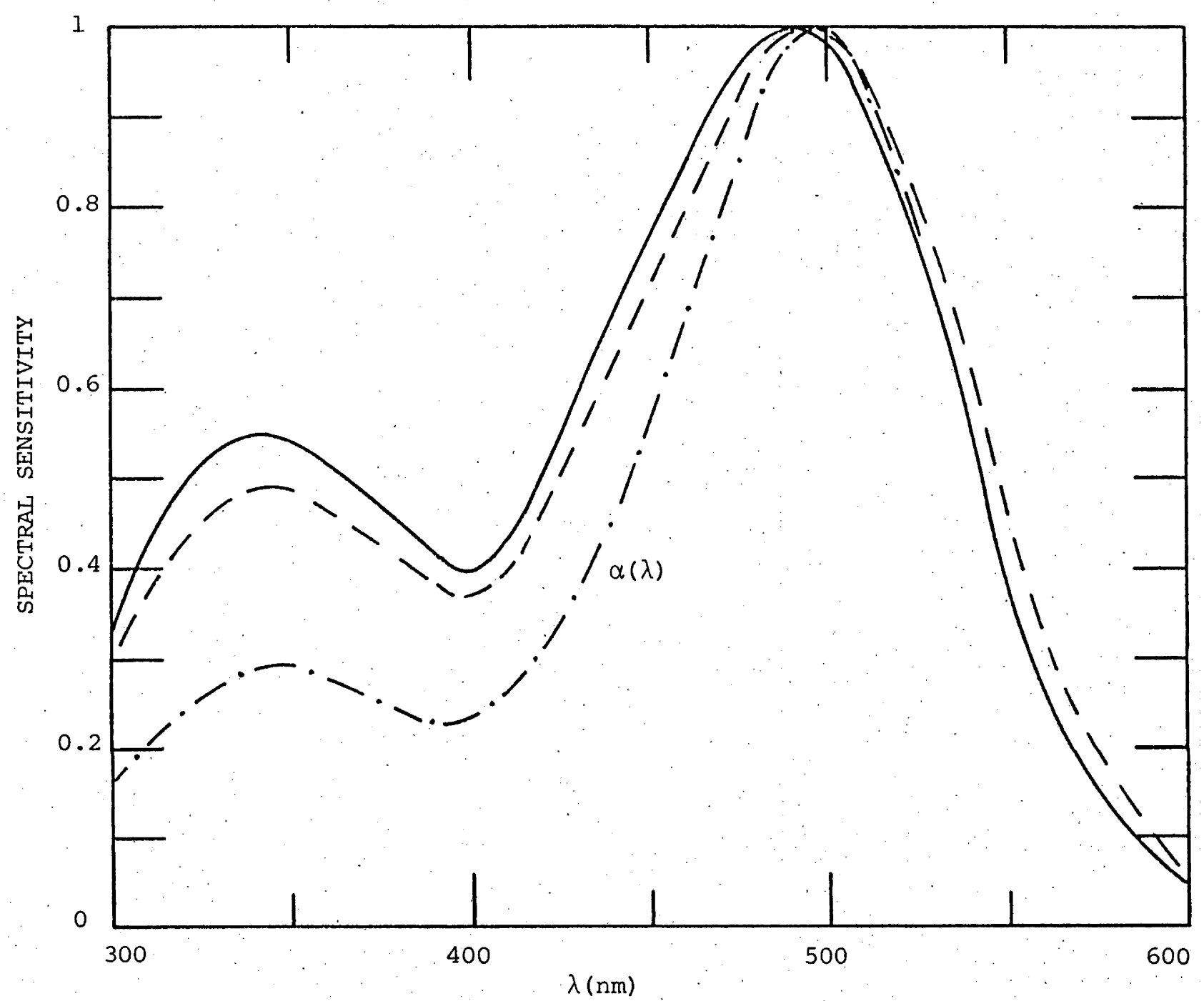

Fig. 7.7. Spectral sensitivity of a rhabdomere in isolation $(-\rightarrow-$, and of $R_{A}$ the illuminated rhabdomere of the coupled identical pair (-). These curves are found by renormalising the curves of Fig. 7.6 to a maximum of

1. The lowest curve is the photopigment absorption $\alpha(\lambda)$ used to calculate the power absorbed. 


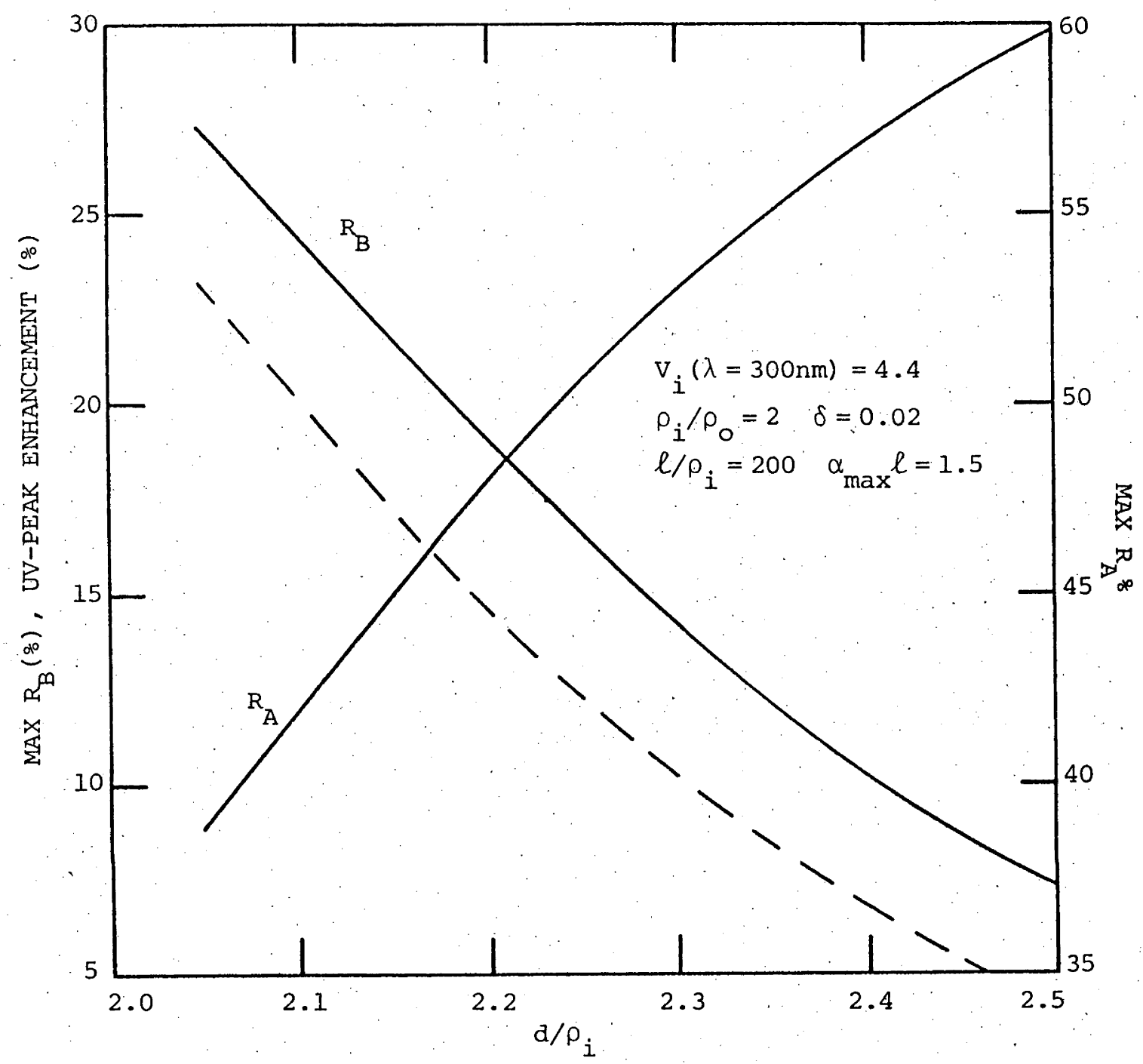

Fig. 7.8. Maximum percentage absorption by the illuminated rhabdomere $\mathrm{R}_{\mathrm{A}}$ and the coupled rhabdomere $\mathrm{R}_{\mathrm{B}}$ as a function of normalised separation $d / p_{i}$. The rhabdomeres are tapered but parallel. $R_{A}$ alone absorbs $65.7 \%$ of the incident light at $\lambda=494 \mathrm{~nm}$ (see Fig. 7.6). The dashed curve is the enhancement of the uv peak $(\lambda=340 \mathrm{~nm})$ due to the crosstalk and is calculated from spectral sensitivity curves like those of Fig. 7.7. 


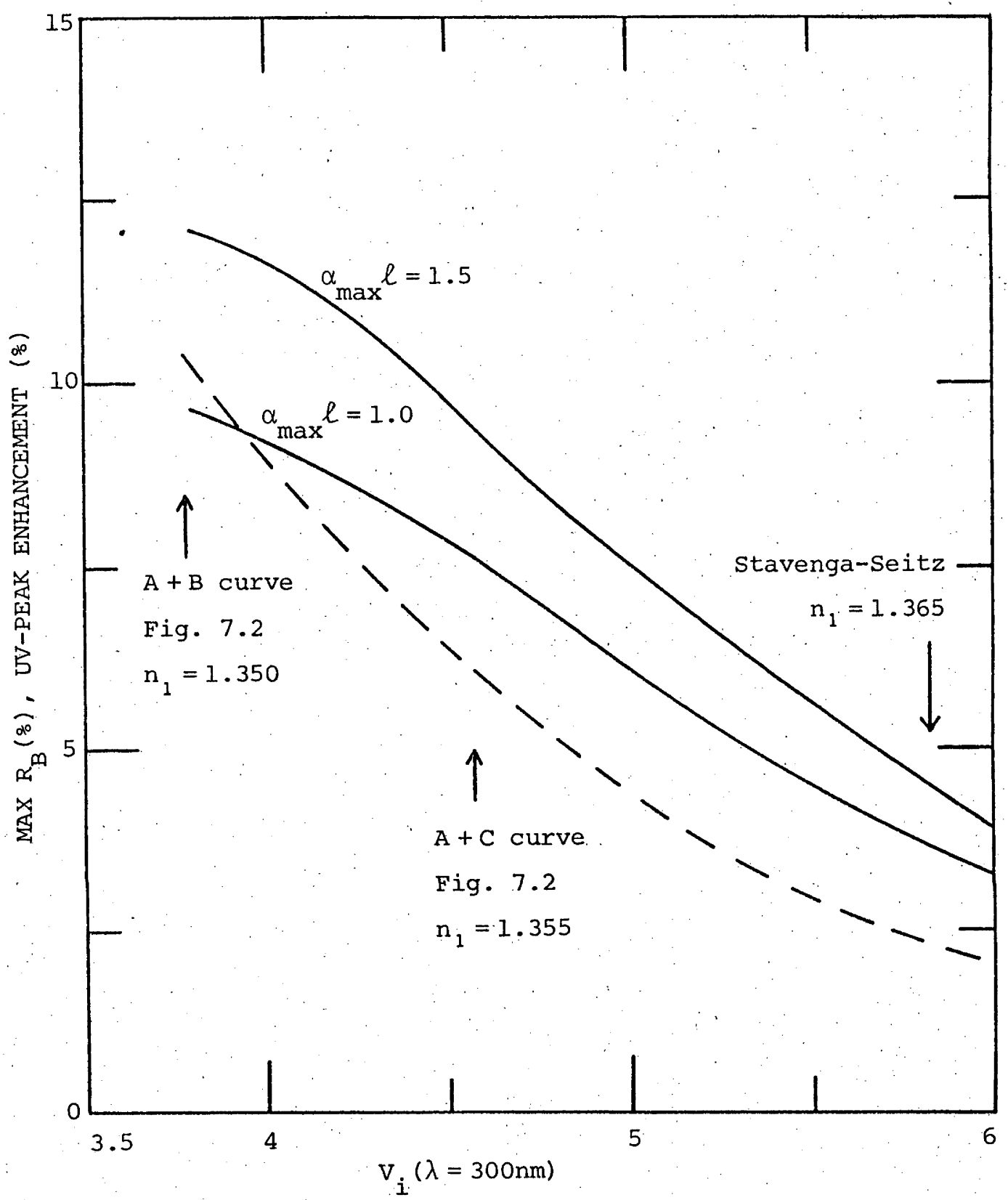

Fig. 7.9. Maximum percentage absorption by the unilluminated rhabdomere $R_{B}$ for two values of the maximum absorption coefficient $\alpha_{\max } \ell=1.0,1.5$, as a. function of $v_{i}(\lambda=300 \mathrm{~nm}) . v_{i}$ is varied by changing $\mathrm{n}_{1}$. The dashed curve again gives the enhancement of the uv peak of $R_{A^{\prime}}$ which is the same for both values of $\alpha_{\max } \ell$. The parameters used are those of Fig. 7.6. 
(a) the maximum power absorbed in $R_{B}$ for two values of $\alpha_{\max } l$, and

(b) the uv-peak enhancement of $R_{A}$, as a function of $v_{i}$, the value of $V$ at the distal end; $v_{i}$ is varied by changing $n_{1}$. The curves cover the possible range of $\mathrm{V}$ as discussed in section 7.4. The uv-peak enhancement is the same for $\alpha_{\max } l=1.0,1.5$. An interesting observation from Figs. $7.8,7.9$ is that except at the lower end of the $\mathrm{V}$ scale, the uvpeak enhancement is directly proportional to the maximum power absorbed in $R_{B}$. Some correlation between the two is obviously expected.

This direct proportionality allows the uv-peak enhancement to be estimated once the maximum absorbed crosstalk power is known.

Although the power absorbed by $R_{B}$ is small, significant power transfer does take place at the longer wavelengths. This is illustrated in Fig. 7.10, which shows how the power at the proximal end of the rhabdomeres is divided between $R_{A}$ and $R_{B}$. The total power at the proximal end $\left(R_{A}+R_{B}\right)$ is given by $P(l)=P(0) e^{-\alpha \bar{n} l}$, where $P(0)$ is the initial power in $R_{A}$ and $\bar{\eta}$ is the average value of $\eta$ for a fly rhabdomere (Snyder and Pask, 1973a). Figure 7.10 shows that with $\lambda=600 \mathrm{~nm}, 72 \%$ of the power $P(\ell)$ at the proximal end of the rhabdomeres is in $R_{B}$ and only $28 \%$ in the illuminated rhabdomere $\mathrm{R}_{\mathrm{A}}$. In the uv region very little power transfer takes place.

We have made several assumptions in the crosstalk calculations for Figs. 7.6-7.10. The first of these is that the two rhabdomeres are identical. As we have shown in chapter 3, incomplete power transfer takes place between $\mathrm{P}_{0_{1}}$ modes on non-identical waveguides, but the initial rate of power transfer is greater than for identical rhabdomeres. Figure 7.11 shows the effect on the crosstalk power at the proximal end of the two rhabdomeres when their radii differ by $\Delta \rho$. Provided that $\frac{\Delta \rho}{\rho}$ 


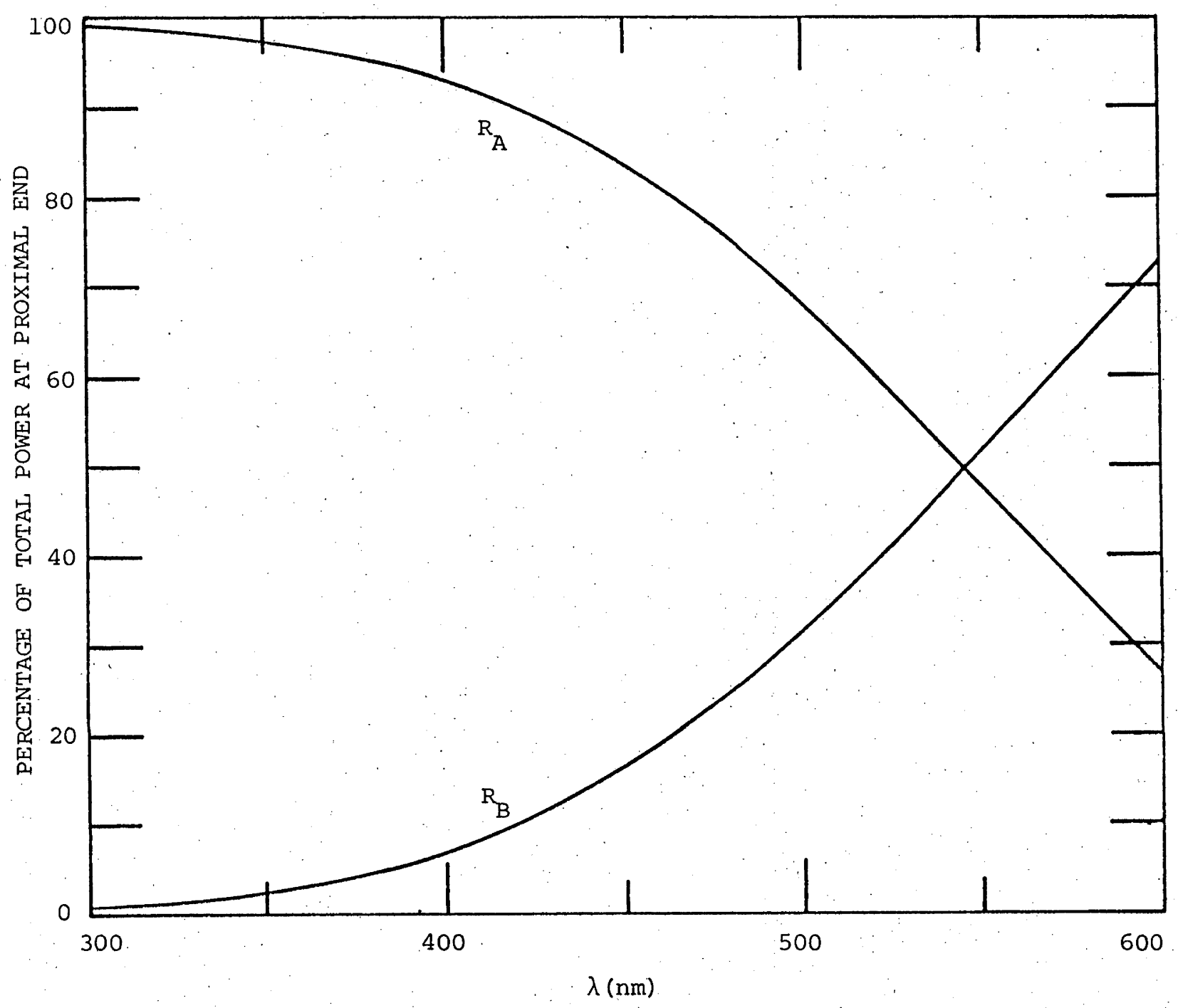

Fig. 7.10. Distribution of the power at the proximal end of two coupled fly rhabdomeres as a function of wavelength. Parameters are those of Fig. 7.6. 


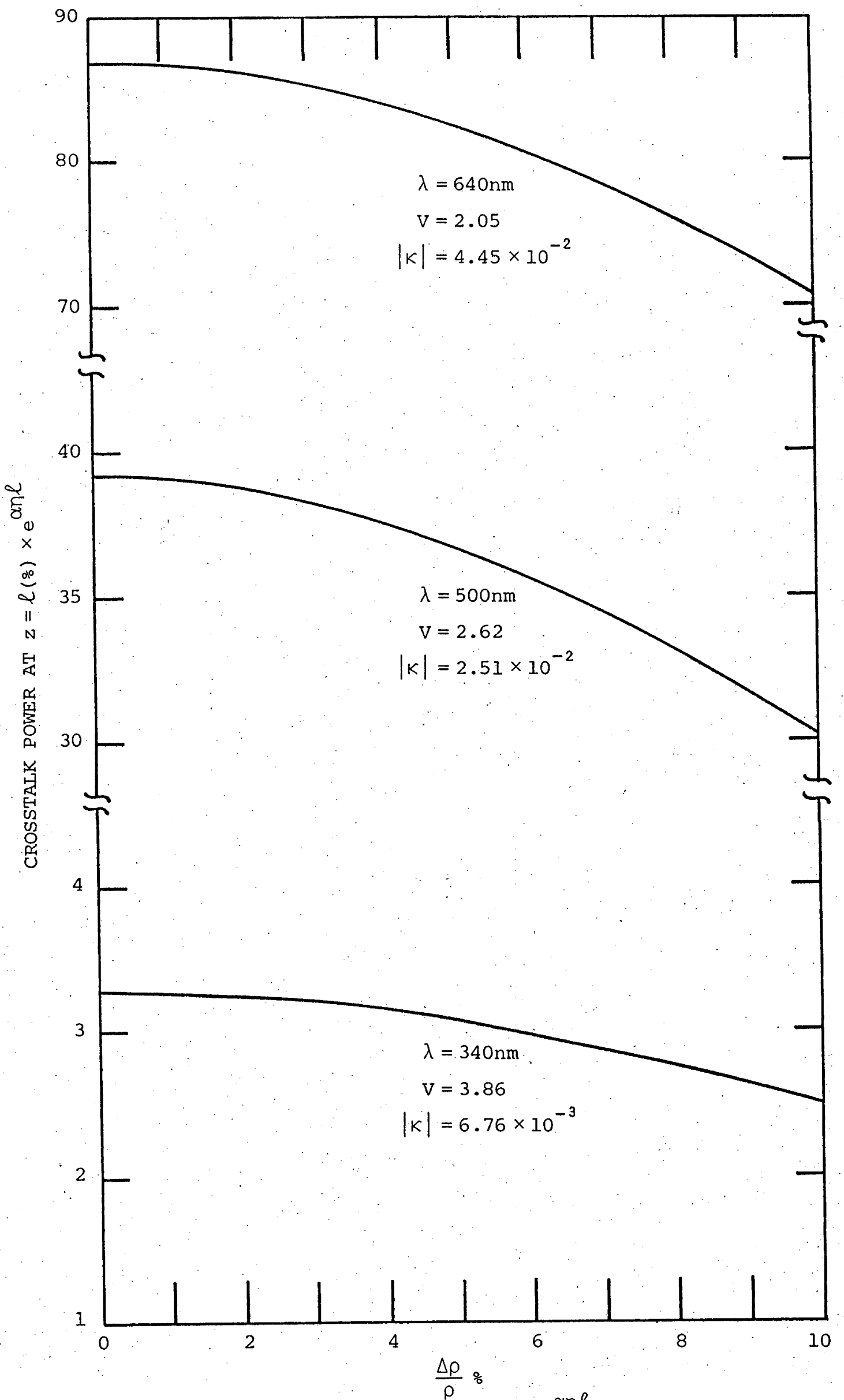

Fig. 7.11. Crosstalk power (power in $R_{B}$ ) $\times e^{\alpha \eta l}$ at the proximal end $(z=\dot{l})$ as a function of the difference $\Delta \rho$ between the radii of the two rhabdomeres. $\ell=200 \mu$. The rhabdomeres are untapered. 
is only a few percent, the fall-off in the power transfer is not great for the rhabdomere length used $(l=200 \mu)$. The effect of the radius difference on the total power absorbed will be even less, because the power transfer takes place initially at a faster rate than when the rhabdomeres are identical. Therefore in the non-identical rhabdomeres, more power (than in identical rhabdomeres) is transferred in the distal region before much absorption has occurred; this tends to compensate for the lessened total power transfer over the length of the rhabdomeres. These considerations apply only when the difference in the radii is small $\left(\frac{\Delta \rho}{\rho}<10 \%\right)$. Both the total power transfer and the absorbed crosstaik power drop off very rapidly as the radius difference is increased beyond this value. This means that crosstalk between the central rhabdomere R7,8 $(\rho=0.5 \mu)$ and any of the peripheral rhabdomeres $R 1-6(\rho=1 \mu)$ is always small at the distal end. The rate of power transfer will be faster at the proximal end where the radii are approximately equal, but here very little power remains to be absorbed, and the separation of $R 7,8$ and $\mathrm{Rl}-6$ is also relatively large.

The mode properties have been calculated assuming a circular cross-section, although the rhabdomeres are obviously more elliptical in shape (Boschek, 1971). This ellipticity should have little qualitative effect on the mode properties, because an elliptical waveguide supports a fundamental $\mathrm{P}_{01}$-like mode (Yeh, 1972), but does raise the problem of which radius and which separation to use. Perhaps the best method is to use centre-to-centre spacing and the value of the radius (and $v$ ) along the line joining the centres. According to wijngaard and Stavenga (1975), the different ways of choosing the separation make little difference to the power transfer, as we found in section 4.4.2 for tapered fibres; the increase in the normalised separation $d / \rho$ with 
decreasing $\rho$ is compensated for by the decrease in V. An interesting comparison would be to use the rhabdomeres of the mosquito Wilhelmia equina, illustrated in Boschek (1971). This has seven identical rhabdomeres of approximately circular cross-section arranged in a regular hexagonal array, with the seventh rhabdomere in the centre. If this arrangement is maintained along the length of the rhabdomeres this rhabdom would provide an ideal subject for crosstalk investigations.

We have calculated crosstalk between pairs of rhabdomeres, whereas in fact the rhabdomeres of Musca are arranged in a trapezoidal. array. The presence of more than one neighbouring rhabdomere causes a faster rate of power transfer (Section 3.6) and will therefore tend to accentuate crosstalk effects (especially the uv-peak enhancement). However we find that, in Musca, the rhabdomeres can usually be grouped into pairs according to their separation. The fact that the rhabdomeres are arranged in a regular array will be of importance however in Wilhelmia.

A further consideration is the effect of the surrounding medium. We have assumed this to be lossless, which should be a good approximation for a dark-adapted eye, in which the retina-cell screening pigment is well away from the rhabdomere boundaries and, in general, outside the depth of penetration. In a light-adapted eye however, the screening pigment moves up to the rhabdomere boundary where it absorbs some of the light travelling along the rhabdomere (Kirschfeld and Franceschini, 1969; Stavenga et al, 1973). Any loss reduces the crosstalk, so that the values for the crosstalk in Figs. 7.6-7.11 will be less for a lightadapted eye. A compensating factor is that the presence of the screening pigment tends to increase $\mathrm{n}_{2}$ therefore decreasing $\mathrm{V}$ (Snyder and Horridge, 1972). 
The taper of the rhabdomeres $1-6$ with length is shown by Boschek (1971) to be slightly parabolic rather than linear as we have assumed. The value for $\mathrm{V}$ in the distal region is therefore larger than we have taken, but the normalised separation $d / \rho$ is reduced, with the overall effect of a small increase in crosstalk in this region, compared with a linear taper (see Section 4.4.2).

All calculations have been for the $\mathrm{P}_{01}$ mode, which is the dominant mode for on-axis excitation. Higher-order modes if present (Section 7.4) exchange power at a faster rate than the $P_{01}$ mode and will therefore tend to increase the crosstalk. The magnitude of this effect depends on the relative initial powers in the modes (see Chapter 6 , Pask and Snyder, 1975a).

We conclude that about $10 \%$ of the initial power in rhabdomere $\mathrm{R}_{\mathrm{A}}$ of Misca is absorbed by a neighbouring rhabdomere $\mathrm{R}_{\mathrm{B}} \cdot$ This value is an estimate only because of the sensitivity of the crosstalk to the separation between the rhabdomeres. An accurate measurement of the separation is necessary for a more exact calculation of the crosstalk.

\subsection{CONSEQUENCES OF CROSSTALK}

An important property of the visual system of an animal is the ability to discriminate fine details of objects in the animal's environment - visual acuity (reviews Kirschfeld, 1969á; Götz, 1972; Goldsmith and Bernard, 1974; Palka and Pinter, 1975; Snyder, 1976). A general rule is the greater the number of photoreceptors per unit area of the retina, the greater the capacity of the eye to resolve fine detail. However because of the diffraction properties of the lens system, there is in general an optimum spacing of photoreceptors for 
resolution of two point sources. Eyes with this optimum spacing are called diffraction-limited (Barlow, 1964; Kirschfeld, 1975). Kuiper (1966) has shown that the fly visual system is not diffraction-limited because of the size of the rhabdomeres. In fact the diffraction limit would correspond to rhabdomeres 1-6 smaller and almost touching (Snyder, 1976). Because the rhabdomere spacing is not set by the diffraction limit we look to other factors and one of these is obviously crosstalk. We have shown in Section 7.6 that touching (and smaller) rhabdomeres in fly would give rise to an unacceptable level of crosstalk.

The limitation on photoreceptor dimensions and spacing is of importance in vertebrate eyes also. From a comparative study of animals with high resolution, Miller and Snyder (1976) have observed that as the outer segments are packed closer together to achieve higher resolution, they become shorter. Optical crosstalk therefore appears to set an upper limit on the length of the outer segments especially in animals with very high resolution, such as eagles and hawks. Further investigations in this area are being carried out at present. Having established that crosstalk is a factor in determining the optimum size and spacing of photoreceptors in fly and in vertebrates, we now consider ways in which photoreceptors may have evolved so as to reduce crosstalk.

(1) Separation. The most effective means of reducing crosstalk between photoreceptors without altering other properties (except perhaps acuity) is to separate them by at least four radii centre-to-centre (see the depth of penetration curves Fig. 7.4 in section 7.4). This appears to be the way in which fly rhabdomeres have evolved specifically 
to counter crosstalk, if the observations of Wijngaard and Stavenga (1975) are correct. They show that the rhabdomeres are relatively close together for a short length at the distal end, thereby achieving reasonable resolution, but they then curve away from each other quite abruptly and only converge again at the proximal end. This peculiar structure if present in the living animal (see section 7.3) obviously reduces the crosstalk substantially, especially in the distal region where most of the light is absorbed. Wijngaard and stavenga calculate a value for the absorbed crosstalk power of about 13\%, but this is for a $300 \mu$ long rhabdomere. However they also use the refractive index $n_{1}=1.365$ (Stavenga, 1974), which gives a higher value for $\mathrm{V}$ than found by Kirschfeld and Snyder (1975). Using the lower value for $V$ will tend to increase their value for the absorbed crosstalk power (Section 7.6, Fig. 7.9)

(2) Length. The shorter the photoreceptors, the less the crosstalk, all other parameters being equal. This factor is important in vertebrate eyes (Miller and Snyder, 1976). However the shorter the photoreceptor, the less the total power absorbed and the lower the absolute sensitivity of the photoreceptor. If, for example, the fly rhabdomeres were spaced at the diffraction-limited values, they would have to be very short to avoid significant crosstalk; this would involve an unacceptable loss of absolute sensitivity (Snyder, 1976).

(3) Tapered rhabdomeres. Why fly rhabdomeres 1-6 taper still remains something of a mystery, although Boschek (1971) has suggested several reasons. The first of these is that the absorption per unit volume remains approximately constant throughout the length of the rhabdomeres. (This has a bearing on the rate of conversion of the photopigment rhodopsin to metarhodopsin along the length of the 
rhabdomeres - review Hamdorf and Schwemer, 1975). However we find the power absorbed per unit volume only remains constant in the proximal half of Rl-6 and has a much larger value at the distal end. About twice as much power is absorbed per unit volume at the distal end than at the proximal end. Although the rate is not constant, it still varies less in a tapered rhabdomere than in one that is not tapered.

The second reason given by Boschek for tapering is that only about half the amount of photopigment is necessary in a tapered fly rhabdomere (initial radius $\rho_{i}$ ) for the same absorption as in an untapered rhabdomere of radius $\rho_{i}$ and of equal length. This value is calculated from the volumes of the two shapes. However Boschek does not take account of waveguide effects in his calculation. The total power absorbed is $1-\exp (-a \bar{n} \ell)$, where $\bar{n}$, the average fraction of modal power within the rhabdomere, varies with the radius (Snyder and Pask, 1973a); Boschek has taken $\bar{n}=1$ for both the tapered and untapered rhabdomeres. $\bar{\eta}$ for a tapered rhabdomere is less than $\eta$ for an untapered rhabdomere of uniform radius $\rho_{i}$, so that for equal absorption we require

$$
\left(\alpha_{\max } \bar{\eta} \ell\right)_{\text {tap }}=\left(\alpha_{\max } \eta \ell\right)_{\text {ut }}
$$

where ut denotes the untapered rhabdomere. Therefore

$$
\frac{\left(\alpha_{\max } l\right)}{\left(\alpha_{\max }\right)_{u t}}=\frac{\eta_{u t}}{\eta_{\text {tap }}}
$$

and to achieve equal absorption by the tapered rhabdomere we must either increase its length $l$ or the concentration of its photopigment $\left(\alpha_{\max }\right)$. The respective volumes (amount of photopigment $\propto$ volume) can be calculated from the lengths, assuming equal concentration of photopigment in the tapered and untapered rhabdomeres. In the Table below we give values for the ratio of the $\alpha_{\max } \ell^{\prime}$ 's necessary for equal. 
absorption at different $v_{i}$, assuming that the taper is that of Rl-6 i.e. $\rho_{i} / \rho_{0}=2$. Also given are the ratios of the volumes of the tapered and untapered rhabdomeres. With equal concentration, the ratio of the volumes is the ratio of the amounts of photopigment. The values for $v_{i}=\infty$ are those found by Boschek for which there are no waveguide effects.

\begin{tabular}{|c|c|c|c|c|}
\hline$v_{i}$ & $\eta_{u t}$ & $\bar{n}_{\text {tap }}$ & $\frac{\left(\alpha_{\max ^{l)}} \operatorname{tap}\right.}{\left(\alpha_{\max }\right)^{l)} \text { ut }}$ & $\frac{\mathrm{Vol}_{\text {tap }}}{\mathrm{Vol}_{\mathrm{ut}}}$ \\
\hline 2 & 0.75 & 0.5 & 1.5 & 88.7 \\
\hline 3 & 0.88 & 0.78 & 1.13 & 66.7 \\
\hline 4 & 0.95 & 0.85 & 1.12 & 65.4 \\
\hline 5 & 0.97 & 0.92 & 1.05 & 61.4 \\
\hline 6 & 0.98 & 0.94 & 1.04 & 60.6 \\
\hline$\infty$ & 1 & 1 & 1 & 58.5 \\
\hline
\end{tabular}

The relative amounts of photopigment vary with the wavelength because of the wavelength dependence of $\mathrm{v}_{i}$. For example, the value of $V_{i}$ for fly $1-6$ at $\lambda=330 \mathrm{~nm}$ is 4 , so that for equal absorption, the tapered rhabdomere requires only about $65 \%$ of the photopigment in the untapered rhabdomere. However at $\lambda=660 \mathrm{~nm}, v_{i}=2$ and the amount is now $89 \%$. We conclude that there is a saving in the amount of photopigment by having tapered rather than untapered rhabdomeres, but because of waveguide effects, the saving is less than predicted by Boschek (1971), and at the longer wavelengths is quite small.

A third reason for tapered rhabdomeres is a reduction of crosstalk. For a given separation and $v_{i}$, there is less crosstalk between tapered rhabdomeres than between non-tapered rhabdomeres. This is illustrated in Fig. 7.12 which shows the power absorbed in two untapered 


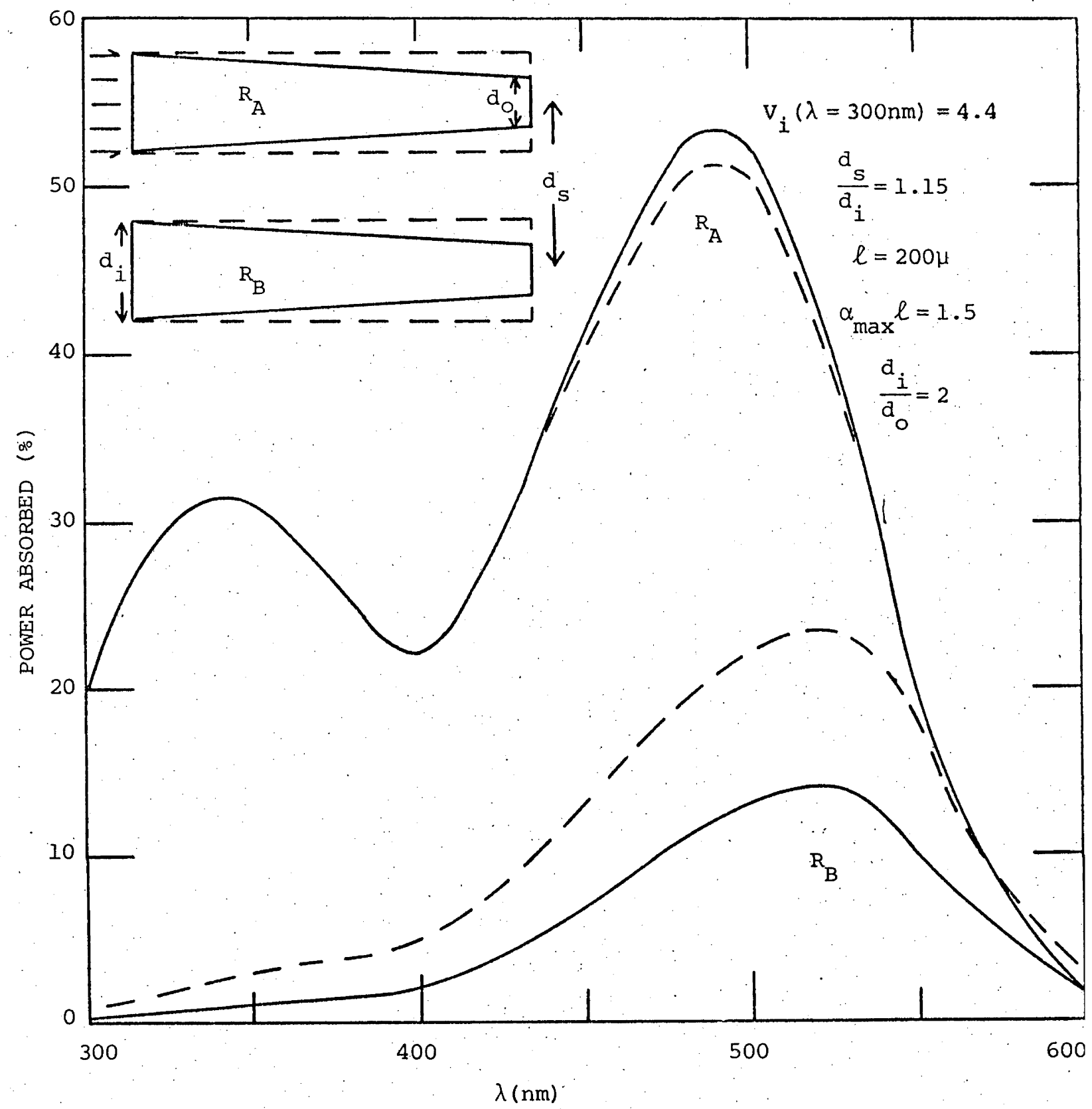

Fig. 7.12. Comparison of the power absorbed in two tapered ( $\longrightarrow$ and in two untapered (- - - rhabdomeres as a function of the wavelength. The total absorption $\left(R_{A}+R_{B}\right)$ in the two untapered rhabdomeres at $\lambda=490 \mathrm{~nm}$ is $72.7 \%$, whereas in the two tapered rhabdomeres it is $65.7 \%$. 
rhabdomeres and in two tapered rhabdomeres with the same separation and initial radius. The important point from Fig. 7.12 is that, although the total absorption (i.e. in both rhabdomeres) in the tapered pair is about 5\% less (at $\lambda=490 \mathrm{~nm}$ ), the power absorbed by the illuminated tapered rhabdomere actually increases, whereas the crosstalk power drops considerably. If there were no crosstalk, the power absorbed in a tapered rhabdomere would be less than in the equivalent untapered rhabdomere (the power absorbed in an isolated rhabdomere is equal to the sum of the powers absorbed in two coupled rhabdomeres). In this case tapering reduces the absolute sensitivity. However because crosstalk does take place, this drop in absolute sensitivity is more than compensated for by the decrease in crosstalk when the rhabdomeres are tapered.

We conclude that, by tapering, the rhabdomeres can considerably reduce crosstalk while maintaining or even increasing their absolute sensitivity compared with untapered rhabdomeres. Whether this is a significant reason for the rhabdomeres to be tapered depends on the magnitude of the crosstalk; the greater the crosstalk, the more reason to taper.

The presence of crosstalk means that the phenomenon of optical coupling, which is important for fused rhabdoms (Snyder et al, 1973), is also relevant to an open rhabdom structure. The absorption of light in a rhabdomere can affect the absorption in a neighbouring rhabdomere. In Musca however, rhabdomeres 1-6, for which crosstalk is important, are of the same spectral type; in this case, differences between the spectralsensitivity curves of the illuminated rhabdomere in the presence of a neighbouring rhabdomere and of an identical rhabdomere in isolation are produced by the wavelength dependence of the crosstalk. The increase in the height of the uv peak caused by crosstalk is an example of lateral 
filtering, which is characteristic of optical coupling.

There is little crosstalk between rhabdomeres of different spectral types (RI-6 and R7,8) in Musca. However if, for example, the seven rhabdomeres of the mosquito. Wi Ihelmia (Boschek, 1971) are not all the same spectral type, lateral filtering due to crosstalk could play an important part in determining their spectral and polarisation sensitivities in exactly the same way as in fused rhabdoms. Lateral filtering could also be of importance in double cones which occur in some vertebrates.

In conclusion, the level of crosstalk between photoreceptors appears to be theoretically within the limits necessary for the photoreceptors to operate independently, although it is difficult to say just what is an acceptable level of crosstalk. At low light levels even a few percent crosstalk may be important for contrast resolution (Miller and Snyder, 1976). Certainly crosstalk represents a limitation on the size and spacing of photoreceptors, and must be considered when predicting the optimum parameters for a visual system (see for example Pask and Snyder, 1975; Snyder, 1976; Miller and Snyder, 1976). Because of crosstalk the peripheral rhabdomeres $\mathrm{Rl}-6$ of the fly rhabdom have had to suffer a loss of acuity in order to maintain a high absolute sensitivity. 


\section{REFERENCES}

Barlow, H.B. (1964), "Physical limits of visual discrimination", in Photophysiology, A.C. Giere ed., vol.II (Academic Press, New York).

Bernard, G.D. (1975), "Physiological optics of the fused rhabdom", in Photoreceptor Optics, A.W. Snyder and R. Menzel eds. (SpringerVerlag, Heidelberg).

Boschek, C.B. (1971)., "On the fine structure of the peripheral retina and lamina ganglionaris of the fly, Musca domestica", Z. Zellforsch. 118,369 .

Braitenberg, V. and Strausfeld, N.J. (1973), "Principles of the mosaic organisation in the visual system's neuropil of Musca domestica L", in Handbook of Sensory Physiology VII/3A, R. Jury ed. (SpringerVerlag, Berlin).

Brücke, E.W. von (1843), Archiv fur Anat. und Physiol. 11, 444: quoted in O'Brien, B. (1946), "A theory of the Stiles and Crawford effect", J. Opt. Soc. Am. 36, 506.

Burkhardt, D. (1964)., "Colour discrimination in insects", Advanc. Insect Physioz. 2,131 .

Dartnall, H.J.A. ed. (1972), Handbook of Sensory Physiology VII/1: Photochemistry of Vision (Springer-Verlag; Berlin).

Dörrscheidt-Käfer, M. (1972), "Die Empfindlichkeit einzelner Photorezeptoren in Komplexauge von Calliphora erthrocephala"

J. Comp. Physiol. 81, 309.

Enoch,'J.M. (1963), "Optical properties of retinal receptors", J. Opt. Soc. Am. 53, 71.

Enoch, J.M. (1967); "Comments on Excitation of waveguide modes in retinal receptors", J. Opt. Soc. Am. 57, 548 .

Franceschini, N. and Kirschfeld, K. (1971), "Etude optique in vivo des éléments photorecepteurs dans 1'oeil composé de Drosophila", Kybernetik 8,1 .

Fuortes, M.G.F. ed. (1972), Handbook of Sensory Physiology VII/2: Physiology of Photoreceptor Organs (Springer-Verlag, Berlin). 
Goldsmith, T.N. and Bernard, G.D. (1974), "The visual system of insects", in Physiology of Insecta, vol. II, M. Rockstein ed. (Academic Press, New York).

Hamdorf, K. and Schwemer, J. (1975), "Photoregeneration and the adaptation process in insect photoreceptors", in Photoreceptor Optics, A.W. Snyder and R. Menzel eds. (Springer-Verlag, Heidelberg).

Horridge, G.A. ed. (1974), The Compound Eye and Vision of Insects (Clarendon Press, Oxford).

Horridge, G.A. (1975), "Arthropod receptor optics", in Photoreceptor Optics, A.W. Snyder and R. Menzel eds. (Springer-Verlag, Heidelberg).

Kirschfeld, K. (1969), "Absorption properties of photopigments in single rods, cones and rhabdomeres", in Processing of optical Data by Organisms and by Machines, w. Reichardt ed. (Academic Press, New York).

Kirschfeld, K. (1969a), "Optics of the compound eye", in Processing of Optical Data by Organisms and by Machines, w. Reichardt ed. (Academic Press, New York).

Kirschfeld, K. (1972), "The visual system of Musca: Studies on optics, structure and function", in Information Processing in the Visual System of Arthropods, R. Wehner ed. (Springer-Verlag, Berlin).

Kirschfeld, K. (1975), "The compound eye of the fly: Differences and analogies to vision in vertebrates", submitted to Science.

Kirschfeld, K. and Franceschini, N. (1969), "Ein Mechanismus zur Steurung des Lichtflusses in den Rhabdomeren des Komplexauges von Musca", Kybernetik, 6 , 13.

Kirschfeld, K. and Snyder, A.W. (1975), "Waveguide mode effects, birefringence and dichroism in fly photoreceptors", in Photoreceptor Optics, A.W. Snyder and R. Menzel eds. (SpringerVerlag, Heidelberg).

Kuiper, J.W. (1966), "On the image formation in a single ommatidium of the compound eye in Diptera", in The Functional Organisation of the Compound Eye, C.G. Bernhard ed. (Pergamon Press, Oxford).

Liebman, P.A. and Entine, G. (1968), "Visual pigments of frog and tadpole", Vision Res. $8,761$.

Melamed, J. and Trujillo-Cenóz, O. (1968), "The fine structure of the central cells in the ommatidia of Dipterans",

J. Ultrastruct. Res. 21,313 .

Miller, W.H. and Snyder, A.W. (1973), "Optical function of human peripheral cones", Vision Res. 13, 2185.

Miller, W.H. and Snyder, A.W. (1976), "Resolving power of eagles and hawks", in preparation. 
Pask, C. and Snyder, A.W. (1975), "Theory of the Stiles-Crawford effect of the second kind", in Photoreceptor Optics, A.W. Snyder and R. Menzel eds. (Springer-Verlag, Heidelberg).

Pask, C. and Snyder, A.W. (1975a), "Angular sensitivity of lensphotoreceptor systems", in Photoreceptor Optics, A.W. Snyder and R. Menzel eds. (Springer-Verlag, Heidelberg).

Reichardt, w. ed. (1969), Processing of Optical Data by Organisms and by Machines (Academic Press, New York).

Sammut, R.A. (1975), "The theory of unbound modes on circular dielectric waveguides", Ph.D. Thesis, Australian National University, Canberra.

Sammut, R.A. and Snyder, A.W." (1974), "Contribution of unbound modes to light absorption in visual photoreceptors",

J. Opt. Soc. Am. 64, 1171.

Seitz, G. (1968), "Der Strahlengang im Appositionsauge von Calliphora erythrocephala (Meig.)", Z. Vergl. Physiol. 59, 205.

Snyder, A.W. (1969a), "Asymptotic expressions for eigenfunctions and eigenvalues of a dielectric or optical waveguide", I.E.E.E. Trans. Microwave Theory Tech. MTT-17, 1130.

Snyder, A.W. (1974b), "Optical properties of invertebrate photoreceptors", in The Compound Eye and Vision of Insects, G.A. Horridge ed. (clarendon Press, oxford).

Snyder, A.W. (1975), "Photoreceptor optics - theoretical principles", in Photoreceptor Optics, A.W. Snyder and R. Menzel eds. (Springer-Verlag, Heidelberg).

Snyder, A.W. (1976), "Acuity of compound eyes", submitted to J. Comp. Physiol.

Snyder, A.W. and Horridge, G.A. (1972), "The optical function of changes in the medium surrounding the cockroach rhabdom", J. Comp. Physiol. 81, 1 .

Snyder, A.W. and Menzel, R. eds. (1975), Photoreceptor Optics (Springer-Verlag, Heidelberg).

Snyder, A.W., Menzel, R. and Laughlin, S.B. (1973), "Structure and function of the fused rhabdom", J. Comp. Physiol. 87, 99.

Snyder, A.W. and Miller, W.H. (1972), "Fly colour vision", Vision Res. 12, 1389.

Snyder, A.W. and Pask, C. (1972b), "A theory for changes in spectral sensitivity induced by off-axis light", J.. Comp. Physiol. 79, 423 . 
Snyder, A.W. and Pask, C. (1973a), "Absorption in conical optical fibres", J. Opt. Soc. Am. 63, 761 .

Snyder, A.W. and Pask, C. (1973b) "Spectral sensitivity of Dipteran retinula cells", J. Comp. Physiol. 84, 59.

Snyder, A.W. and Pask, C. (1973C), "The Stiles-Crawford effect explanation and consequences", Vision Res. 13, 1115.

Stavenga, D.G. (1974), "Visual receptor optics rhodopsin and pupil in fly retinula cells", Thesis, Groningen.

Stavenga, D.G. (1975), "Optical qualities of the fly eye - an approach from the side of geometrical, physical and waveguide optics", in Photoreceptor Optics, A.W. Snyder and R. Menzel eds. (Springer-Verlag; Heidelberg).

Stavenga, D.G., Zantema, A. and Kuiper, J.W. (1973), "Rhodopsin processes and the function of the pupil mechanism in flies", in Biochemistry and Physiology of Visual Pigments, H. Langer ed. (Springer-Verlag, Berlin).

Toraldo di Francia, G. (1948), "Per una teoria dell'effeto StilesCrawford", Il Nuovo Cimento $5,589$.

Trujillo-Cenóz, O. (1972), "The structural organisation of the compound eye in insects", in Handbook of Sensory Physiology viI/2, M.G.F. Fuortes ed. (Springer-Verlag, Berlin).

Trujillo-Cenóz, O. and Bernard, G.D. (1972), "Some aspects of the retinal organization of Sympycnus lineatus Loew (Diptera; Dolichopodidae)", J. Ultrastruct. Res. 38, 149.

Trujillo-Cenóz, O. and Melamed, J. (1966), "Electron microscope observations on the peripheral and intermediate retinas of Dipterans", in The Functional Organisation of the Compound Eye, C.G. Bernhard ed. (Pergamon Press, oxford).

Varela, F.G. and Wiitanen, W. (1970), "The optics of the compound eye of the honeybee (Apis mellifera), J.Gen. Physiol. $\underline{55}, 33.6$.

Wehner, R. ed. (1972), Information Processing in the Visual System of Arthropods (Springer-Verlag, Berlin).

Wijngaard, W. and Heyker, H. (1975), "Optical interaction between retinal receptors", in Photoreceptor Optics, A.W. Snyder and R. Menzel eds. (Springer-Verlag, Heidelberg).

Wijngaard, W. and Stavenga, D.G. (1975), "On optical crosstalk between fly rhabdomeres", Biol. Cybemetics 18, 61.

Yeh, C. (1972); "Noncircular dielectric waveguides", in Optical Waveguides, N.S. Kapany and J.J. Burke (Academic Press, New York), Appendix A. 
CHAPTER $\cdot 8$

PROPAGATION OF LIGHT IN TWISTED MEDIA:

ABSORPTION AND BIREFRINGENCE IN TWISTED PHOTORECEPTORS

\subsection{OUTLINE}

A simple coupled-mode theory is used to describe the propagation of light in a slowly twisting anisotropic medium; expressions are found for the ellipticity and orientation of the polarisation ellipse. In general the light is elliptically polarised and the polarisation ellipse rotates as the light travels through the medium. In two limits incident linearly polarised light remains linearly polarised; i.e. when

(1) the twist rate is relatively large and the anisotropy. small, in which limit there is a small rotation of the direction of polarisation;

(2) the twist rate is small and the anisotropy large. If the light is polarised in one of two orthogonal directions (corresponding to the optic axes of the untwisted medium), the direction of polarisation rotates at the same rate as the medium twists i.e. the direction of polarisation follows the twist.

When a linearly birefringent medium is twisted, its birefringence, as measured with a crossed polariser-analyser, is a complicated function. of the twist angle and the actual birefringence. This is because there are no optic axes in a twisted medium. The coupled-mode formalism is used to find this relationship. 
The second part of the chapter is devoted to an analysis of twisted photoreceptors. We find that

(1) the polarisation sensitivity of rhabdomeres in a fused rhabdom, such as in ant or bee, is reduced by the twisting. A substantial reduction can occur if the dichroic ratio of the microvilli is high. Twisting, in conjunction with electrical coupling, could explain why the measured polarisation sensitivity is lower than predicted;

(2) the direction of polarisation of the incident light for maximum absorption in a twisted rhabdomere is not, in general, parallel to the microvilli axis at the distal end, but at approximately $\Omega / 2$ to it, where $\Omega$ is the total twist angle for the rhabdom;

(3) twisting of the rhabdom can reduce the enhancement of the polarisation sensitivity of the ninth cell in ant or bee; this enhancement is due to the polarisation-filtering effect of the rhabdomere distal to the ninth cell. However, even with twisting, there is still sufficient enhancement to explain the measured values of the polarisation sensitivity;

(4) twisting also decreases the polarisation sensitivity of fly-type rhabdomeres. A twisted fly rhabdomere could therefore have a high dichroic ratio but a low polarisation sensitivity. Twisting also increases the absorption of unpolarised light, but we find that a twisted rhabdomere with a high dichroic ratio still absorbs significantly less unpolarised light than a rhabdomere (twisted or untwisted) with a low dichroic ratio. We conclude that twisting of the rhabdomeres in fly does not alter the basis on which a value $\Delta=2$ for the dichroic ratio is predicted (Snyder and Laughlin, 1975).

(5) the measured birefringence of a twisted fly rhabdomere 
has no simple relationship to the actual birefringence. By choosing suitable values for the parameters we find a decrease in the measured birefringence with length, as found by Kirschfeld and Snyder (1975), but with other values the measured birefringence can increase.

\subsection{INTRODUCTION}

Several interesting properties are exhibited by an anisotropic medium that twists about the direction of the advancing wave. In general an incident linearly polarised wave becomes elliptically polarised, and the axes of the polarisation ellipse rotate as the wave propagates through the medium. This rotation is form optical rotation (Stokes, 1963), due to the macroscopic structure of the medium, rather than to the optical activity of the molecules. The theory is well-known, having been developed to describe light propagation through liquid crystals, in which the rate of twisting is generally large (De Vries, 1951; Marathay, 1971; Berreman 1972, 1973).

Our interest in twisted media arises from a determination of the absorption of polarised light in twisting insect photoreceptors, in which the twist rate is small (see section 8.6). For this case an alternative formulation using coupled-mode theory displays most easily the effect of twisting on such properties of the medium as dichroic absorption and birefringence. The theory predicts elliptic polarisation and rotation of the polarisation ellipse in general, but in one limit we find that a linearly polarised wave remains linearly polarised and rotates at the same rate as the medium twists. A further possible application of this slow-twist method may be to optical waveguides with a crystalline core (Hu and Whinnery, 1974). 


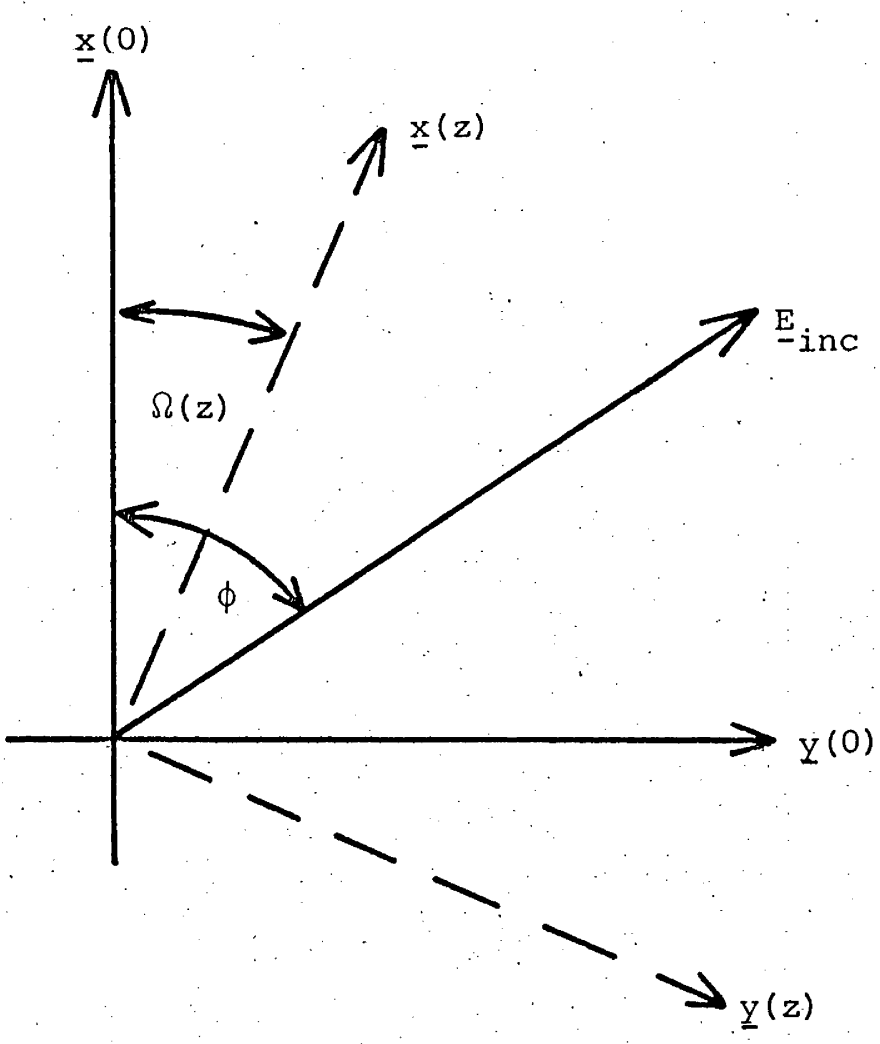

Fig. 8.1

The situation for a twisted medium is shown in Fig. 8.1.

Waves propagate in the $z$ direction, and at any position $z$ the fields can be resolved into two plane waves, one with $\underline{\mathrm{E}}$ parallel to $\underline{x}(z)$, the other with $\mathrm{E}$ parallel to $\underline{\underline{y}}(\mathrm{z})$, where $\underline{\mathrm{x}}(\mathrm{z})$ and $\underline{\mathrm{y}}(\mathrm{z})$ are axes rotating with the twist. The twisting of the medium causes these plane waves to interchange their energy (couple) as they propagate. By using these twisting plane waves, rather than the elliptically polarised normal modes, we can include linear birefringence and dichroic absorption in a simple and intuitive way.

The coupled-mode expressions for the fields are derived in Section 8.3 , followed in section 8.4 by an analysis of the polarisation properties - the ellipticity and rotation of the axes of the polarisation ellipse - and the absorption of light propagating through a twisted, birefringent, dichroic medium. 
The birefringence of a medium is an important indicator of its anisotropic structure. In Section 8.5, we show that the twisting of the medium alters the measured value of the birefringence, and derive a relationship between the measured and the actual birefringence.

The application of the theory to light absorption in twisted photoreceptors is introduced in section 8.6.

\subsection{A COUPLED-MODE THEORY OF LIGHT PROPAGATION IN A TWISTED ANISOTROPIC . MEDIUM}

Consider a stack of identical linearly birefringent plates, characterised by refractive indexes $n_{x^{\prime}} n_{y}$ along the optic axes $x, y$ of each plate, and of thickness $\delta z$. The axes of each plate are rotated. $\delta \Omega$ with respect to the axes of the neighbouring plates. Two such plates. are shown in Fig. 8.2.

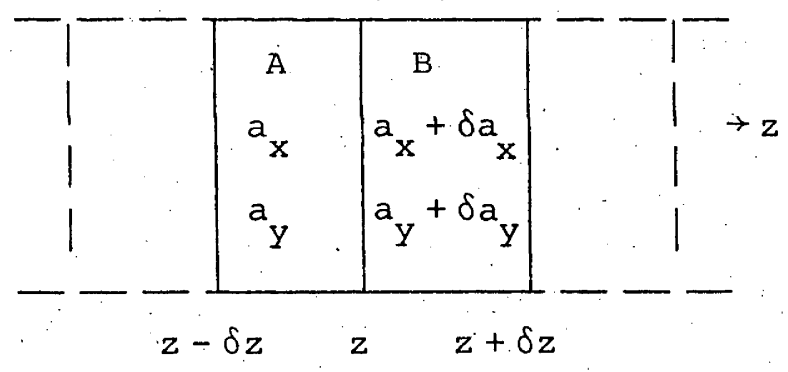

Fig. 8.2

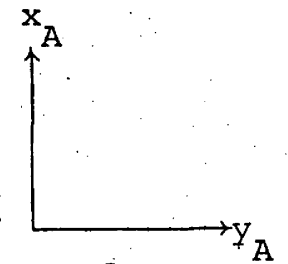

A

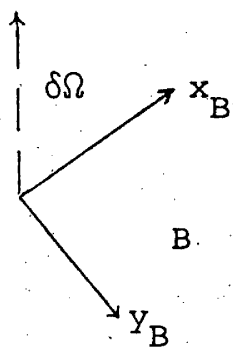

The electric fields propagating in the $+z$ direction in the two plates are given by expressions of the form

$$
\begin{aligned}
& \underline{E}_{A}(z)=a_{x} \underline{x}_{A} e^{i \bar{\beta}_{x} z}+a_{y} \underline{y}_{A} e^{i \bar{\beta} y^{z}} \\
& \underline{E}_{B}(z)=\left(a_{x}+\delta a_{x}\right) \underline{x}_{B} e^{i \bar{\beta}_{x} z}+\left(a_{y}+\delta a_{y}\right) \underline{y}_{B} e^{i \bar{\beta} y^{z}},
\end{aligned}
$$

where $\bar{\beta}_{x}, \bar{\beta}_{Y}$ are the propagation constants corresponding to the refractive indexes $n_{x^{\prime}} n_{y}$. The two sets of axes are related by 


$$
\begin{aligned}
& \underline{x}_{A}=\underline{x}_{B} \cos \delta \Omega-\underline{y}_{B} \sin \delta \Omega \\
& \underline{y}_{A}=\underline{x}_{B} \sin \delta \Omega+\underline{y}_{B} \cos \delta \Omega
\end{aligned}
$$

We now match $(8.1),(8.2)$ at the boundary between the two plates and take the limit $\delta z \rightarrow 0$. We assume a linear twist

$$
\Omega=\xi_{z}
$$

and put

$$
\begin{aligned}
& A_{X}(z)=a_{X}(z) e^{i \bar{\beta}_{X} z} \\
& A_{Y}(z)=a_{Y}(z) e^{i \bar{\beta}_{y} z} .
\end{aligned}
$$

This leads to the coupled-mode equations

$$
\begin{aligned}
& \frac{d A_{x}(z)}{d z}-i \bar{B}_{x} A_{x}(z)=\xi A_{y}(z) \\
& \frac{d A_{y}(z)}{d z}-i \bar{B}_{Y} A_{y}(z)=-\xi A_{x}(z)
\end{aligned}
$$

In order to be able to neglect the interchange of energy between forward and backward waves, we have assumed that $|\xi|<<\left|\bar{\beta}_{x}\right|,\left|\bar{\beta}_{y}\right|$ and that $\left|\frac{\bar{\beta}_{x}-\bar{\beta}_{y}}{\bar{\beta}_{x}+\bar{\beta}_{y}}\right| \ll 1$ (Chapter 2; Louisell, 1960). Equations (8.8), (8.9) describe the propagation of plane waves travelling in the $+z$ direction through a continuously twisting medium with a twist rate of $\xi$ radians per unit length (about the $z$ axis), and propagation constants $\bar{\beta}_{x}, \bar{\beta}_{y}$ along the (twisting) coordinate axes $\underline{x}(z), \underline{y}(z)$. A similar pair of coupled equations describes waves travelling in the $-z$ direction. Note that if $\bar{\beta}_{x}, \bar{\beta}_{y}$ are real, power is conserved by $(8.8)$, (8.9)

Solution of these equations is straightforward (Chapter 2), giving for the field at position $z$ 


$$
\underline{E}(z)=A_{X}(z) \underline{x}(z)+A_{y}(z) \underline{y}(z),
$$

where

$$
\begin{aligned}
A_{X}(z) & =\{p \cos (\xi z / \sqrt{F})+\sqrt{F}(i p \bar{x}+q) \sin (\xi z / \sqrt{F})\} e^{i \bar{\beta}_{s} z} \\
A_{y}(z) & =\{q \cos (\xi z / \sqrt{F})-\sqrt{F}(p+i q \bar{x}) \sin (\xi z / \sqrt{F})\} e^{i \bar{\beta}_{s} z}, \\
\bar{\beta}_{S} & =\frac{1}{2}\left(\bar{\beta}_{x}+\bar{\beta}_{y}\right) \\
\bar{X} & =\frac{\bar{\beta}_{x}-\bar{\beta}_{y}}{2 \xi} \\
F & =\left(1+\bar{x}^{2}\right)-1 \\
p & =A_{x}(0) \\
q & =A_{y}(0),
\end{aligned}
$$

and we take

$$
\frac{1}{2}\left[|p|^{2}+|q|^{2}\right]=1 \text {. }
$$

If the incident field is linearly polarised at angle $\phi$ to $\underline{x}(0)$, $p=\sqrt{2} \cos \phi$ and $q=\sqrt{2} \sin \phi$. We allow for lossy media by writing (see Chapter 6)

$$
\bar{\beta}_{j}=\beta_{j}+i \alpha_{j} / 2: j=x \text { or } y,
$$

where $\beta_{j}=\frac{2 \pi n}{\lambda}, \alpha_{j}$ is the absorption coefficient for light polarised in the $j$ th direction and we have assumed $\alpha_{j} \ll \beta_{j}$. In a dichroic medium $\alpha_{x} \neq \alpha_{y}$. The rotating axes $\underline{x}(z), \underline{y}(z)$ are given by

$$
\begin{aligned}
& \underline{x}(z)=\underline{x}(0) \cos \xi z+\underline{y}(0) \sin \xi z \\
& \underline{y}(z)=-\underline{x}(0) \sin \xi z+\underline{y}(0) \cos \xi z .
\end{aligned}
$$

The power $P(z)$ at position $z$ is given by 


$$
P(z)=\frac{3}{2}\left[\left|A_{X}(z)\right|^{2}+\left|A_{Y}(z)\right|^{2}\right]
$$

In the limit of no twist $(x \rightarrow \infty)$, the expressions for the field reduce to those given by snyder (1973) and Täuber (1974).

\section{Normal Modes}

The normal modes of the twisted system can be found by diagonalising the coupled-mode equations (Section 2.6). For a lossless medium the normal modes are given by

$$
\psi_{1,2}(z)=\left[\underline{x}(z)-i \tau_{1,2} y(z)\right] e^{i \beta_{s} z} e^{ \pm i \xi z / \sqrt{F}},
$$

where the upper (lower) sign in the exponent refers to mode 1 (2). The ellipticities (the ratio of the lengths of the axes of the polarisation ellipse) $\tau_{1,2}$ are given by

$$
\begin{aligned}
\tau_{1} & =x-\sqrt{1+x^{2}} \\
\tau_{2} & =x+\sqrt{1+x^{2}} \\
& =-1 / \tau_{1}
\end{aligned}
$$

Therefore the normal modes are elliptically polarised with axes along the $\underline{x}(z), \underline{y}(z)$ directions, i.e. the polarisation ellipses of the two modes rotate with the twist. The ellipticities of the two modes are the same, but their major axes are perpendicular and the $\mathrm{E}$ vectors rotate in opposite directions. The modes propagate at different velocities because of the $\pm \xi_{z} / \sqrt{F}$ in the exponent. In the limit $x \ll 1$, the normal modes become circularly polarised; when $x \gg 1$ they are linearly polarised and identical to the twisting modes (see section 8.4). The normal modes have also been derived by Marathay (1971) and by Stokes (1963), who uses an interesting and intuitive method.

${ }^{*} \mathrm{x}$ is the real part of $\bar{x}$. 
Having found the normal modes we can of course use them to analyse any particular problem by finding how much power is initially excited in each mode, following them through the twisted medium and adding them together at the end. However the fact that the normal modes are elliptically polarised makes it more difficult to take account of linear birefringence and dichroism in an intuitive. way. We are also mainly interested in linearly polarised incident light, so that we choose to analyse twisting media using the twisting modes.

\section{Twisted Waveguides}

We have formulated our theory of light propagation in twisted media assuming, that there are no waveguide effects i.e. that $\eta=1$. If the twisted medium is a waveguide, with an isotropic cladding e.g. a twisted photoreceptor, the problem becomes more complicated. For the purpose of calculating absorption we can replace $\alpha$ by $n \alpha$, but this does not take full account of the fact that some of the field of the waveguide is propagating in the isotropic cladding, whereas the field inside the waveguide sees a twisted anisotropic medium. (If a medium is isotropic, twisting has no effect on the fields). For example, we would expect less rotation of the field with the twist if a significant proportion of the field is outside the waveguide. For an account of light propagation in anisotropic (but untwisted) waveguides, see for example Marcuse (1975).

\subsection{PROPERTIES OF LIGHT IN A TWISTED MEDIUM}

\subsubsection{Polarisation Parameters}

Light passing through a twisted medium is in general elliptically polarised, and both the ellipticity $\sigma$ and the angle $\psi$ between the major axis of the polarisation ellipse and the rotating $\underline{x}(z)$ axis are functions 
of $z$ (see Fig. 8.1).* The expressions for $\sigma$ and $\psi$ are well-known (e.g. Born and Wolf, 1970; Clarke and Grainger, 1971), and in our. notation are

$$
\begin{aligned}
\tan 2 \psi & =\frac{2 \operatorname{Re}\left(A_{x} A_{y}^{*}\right)}{\left|A_{x}\right|^{2}-\left|A_{y}\right|^{2}} \\
\sigma & =\tan \left\{\frac{1}{2} \sin ^{-1}\left[\frac{2 \operatorname{Im}\left(A A^{A}\right)^{*}}{|A|^{2}+|A|^{2}}\right]\right\}
\end{aligned}
$$

For incident light linearly polarised at angle $\phi$ to $x(0)$, and assuming no $\operatorname{loss}\left(\frac{1}{2}\left[\left|A_{x}\right|^{2}+\left|A_{y}\right|^{2}\right]=1\right),(8.26)$ and $(8.27)$ reduce to

$$
\begin{aligned}
\tan 2 \psi & =\frac{\sin 2 \phi \cos (2 \xi z / \sqrt{F})-\sqrt{F} \cos 2 \phi \sin (2 \xi z / \sqrt{F})}{F \cos 2 \phi\left[\cos (2 \xi z / \sqrt{F})+x^{2}\right]+\sqrt{F} \sin 2 \phi \sin (2 \xi z / \sqrt{F})} \\
\sigma & =\tan \left\{\frac{1}{2} \sin ^{-1}[2 \times \sqrt{F} \sin (\xi z / \sqrt{F})\right. \\
& \times\{\sin 2 \phi \cos (\xi z / \sqrt{F})-\sqrt{F} \cos 2 \phi \sin (\xi z / \sqrt{F})\}]\}
\end{aligned}
$$

The sign of $\sigma$ gives the sense of rotation of the electric vector around the ellipse: $\sigma>0$ corresponds to right-hand elliptically polarised light (Clarke and Grainger, 1971). Note that $\psi \pm n \frac{\pi}{2}$ also satisfies (8.28), and therefore solutions of $(8.28)$ give the angle between $\underline{x}(z)$ and either axis of the polarisation ellipse. To choose the correct $\psi$ we must usually follow the polarisation ellipse as it propagates through the medium. The angle $\psi_{f}$ between the major axis of the ellipse and the $x(0)$ axis, which is fixed in space, is given by

\footnotetext{
*The ellipticity is defined as the ratio of the lengths of the minor and major axes of the polarisation ellipse. The usual symbol is $\eta$ but here we use $\sigma$ to avoid confusion with the waveguide parameter. $0 \leqslant \sigma \leqslant 1$.
} 
$\psi_{f}=\xi_{z}+\psi$

The results (8.28), (8.29) agree with those found from the differential-equation formulation of Azzam and Bashara (1972), and can also be derived from the normal modes, although the algebra is more complicated.

For the remainder of this section we assume that the incident field is linearly polarised (LP) at an angle $\phi$ to $\underline{x}(0)$, and that the medium is lossless (except in Section 8.4.2). Extension to an arbitrary initial field and to absorbing media (see section 8.6) is straightforward. In general an initially LP field becomes elliptically polarised, and the polarisation ellipse rotates as the field propagates through a twisted birefringent medium. This rotation is form optical rotation, which is due to the structure of the medium rather than to the presence of optically active molecules (Stokes, 1963). There are two cases of interest for which the field can remain approximately linearly polarised for all z:

(1) $\mathrm{x}=\frac{\Delta \beta}{2 \xi}>\mathrm{i}$. In this limit the normal modes are approximately linearly polarised, but still have a z-dependent phase difference $(2 \xi z / \sqrt{F}=\Delta \beta z+O(1 / X))$. To order $1 / X$, the normal modes are the same as the twisting modes. If we expand $(8.28)$, (8.29) in $1 / x$, the zerothorder expressions for $\psi, \sigma$ are those of an untwisted medium (but $\psi$ is still defined with respect to the rotating coordinate system), i.e.

$$
\begin{aligned}
\tan 2 \psi & =\tan 2 \phi \cos \Delta \beta z+O(I / x) \\
\sigma & =\tan \left[\frac{1}{2} \sin ^{-1}(\sin 2 \phi \sin \Delta \beta z)\right]+0(1 / x)
\end{aligned}
$$

and $\psi_{f}=\psi+\Omega$, where $\Omega=\xi z$. In general the field is elliptically polarised because of the phase difference between the LP normal modes. However if only one of the normal modes is excited $(\phi=0, \pi / 2)$, the 
field remains LP for all $z$, and the direction of polarisation rotates with the twist $\left(\psi_{\mathrm{f}}=\Omega\right)$. This limit was discussed by Mauguin (1911), but does not seem to have appeared in the literature since. Equations (8.31), (8.32) are a good approximation to the full expressions (8.28), (8.29) when $x \gtrsim 10$.

In Fig. 8.3 we show $\sigma$ as a function of $x$, calculated from (8.29), for several twist angles $\Omega$; the envelope of the maxima of $\sigma$ is also illustrated. For $x \geqslant 1$, the envelope falls off approximately as $I / X$, so that if $x \geqslant 10, \sigma \leqslant 0.1$. When $x>1$, the angle $\psi_{f}$ oscillates about $\Omega$, with the amplitude of the oscillations decreasing as $\mathrm{X}$ increases.

(2) $\frac{\Delta \beta}{2 \xi} \ll 1$. In this limit, discussed in detail by stokes (1963), the normal modes are approximately oppositely circularly polarised. The resultant field is therefore approximately linearly polarised and the direction of polarisation rotates slowly as the field propagates, owing to the different velocities of the normal modes. From (8.28), (8.29), assuming $x \ll 1$, and $x^{2} \Omega \ll 1$,

$$
\begin{aligned}
& \psi_{\mathrm{f}} \approx-\frac{\mathrm{x}^{2} \Omega}{2}\left[1-\frac{\sin 2 \Omega}{\Omega}+\frac{\sin 4 \Omega}{4 \Omega}\right] \quad \phi=0^{\circ} \\
& \sigma \approx x \sin \Omega \sin (2 \phi-\Omega) \ll 1 .
\end{aligned}
$$

The behaviour of $\sigma$ in the small $x$ limit can also be seen in. Fig. 8.3. The envelope of the maxima goes approximately as $\mathrm{x}$ for $\mathrm{x} \leqslant 1$, so that when $x \ll 1$, the field is approximately LP for all $z$. Because $\mathrm{x}^{2} \Omega \ll i$, the rotation of the ellipse about its initial position is small. Stokes (1963) discusses the $\mathrm{x} \ll 1$ limit and finds that when $\phi=0^{\circ}$,

$$
\psi_{f} \approx-\frac{x^{2} \Omega}{2}
$$




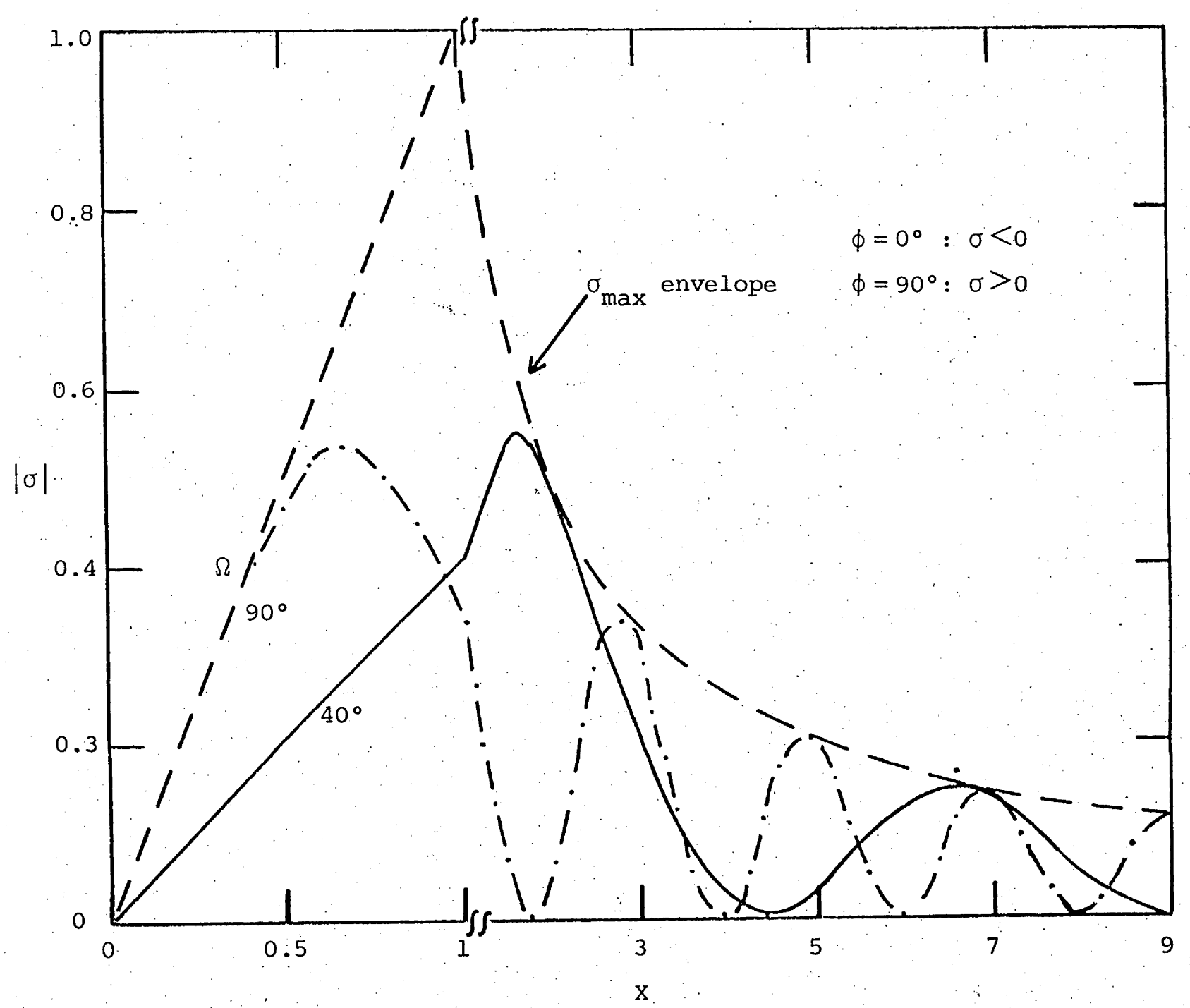

Fig. 8.3. Dependence of the ellipticity $\sigma$ on the parameter $x$, with $\Omega$ fixed i.e. $\ell \Delta \beta$ is varied. Two values of $\Omega$ are shown, together with the envelope of the $\sigma$ curves. $\phi=0^{\circ}$ or $90^{\circ}$. 
This agrees with (8.33), but only in the limit of large $\Omega$. The reason for the discrepancy at small $\Omega[(8.33)$ gives a positive rotation for $\Omega<61^{\circ}$ whereas stokes' rotation is always negative] is not clear. One difference between the two approaches is that Stokes assumes exactly circularly polarised normal modes for his calculations, whereas we retain the small ellipticity. However the difference in the sign of the rotation remains even as $x \rightarrow 0$. As our result is the same as that of Azzam and Bashara (1972), we can only conclude that there are other unstated assumptions in Stokes' calculations.

In between the two limiting cases, the ellipticity $\sigma$ and the angle of rotation $\psi_{f}$ are oscillating functions of $x . \sigma$ oscillates between 0 (linearly polarised field) and the maxima envelope, and reaches a maximum value of 1 (circularly polarised field) only when $x=1$ and $\sqrt{2} \Omega=(2 n+1) \frac{\pi}{2} n=0,1,2, \ldots$ At these points there is a jump of $90^{\circ}$ in $\psi_{f}$ because the major axis of the polarisation ellipse becomes the minor axis (and vice versa) on passing through circular polarisation.

In Figs. 8.4, 8.5 we plot $\sigma$ and $\psi_{f}$ as functions of $\Omega=\xi z$ for a fixed value of $\ell \Delta \beta$; these curves show the effect of different rates of twist $\xi$ on the parameters of the polarisation ellipse. In Fig. 8.4, because $\mathrm{x}=\frac{\ell \Delta \beta}{2 \Omega}, \sigma \rightarrow 0$ for both small and large $\Omega$ (the two limits discussed above), and in between is an oscillating function. Circular polarisation occurs when $\sqrt{2} \Omega=(2 n+1) \frac{\pi}{2}$ and $\ell \Delta \beta=(2 n+1) \frac{\pi}{\sqrt{2}}$ $=2.22,6.66,11.11, \ldots$

Figure 8.5 shows the asymptotic behaviour of $\psi_{f}$ : when $\ell \Delta \beta$ is small and $\Omega$ large $(x \ll 1), \psi_{f} \approx 0$, whereas in the opposite limit $x \gg 1, \psi_{f} \approx \Omega$ and the polarisation ellipse follows the twist (this is only true when $\phi=0$ ). The discontinuity in $\psi_{f}$ on passing through 


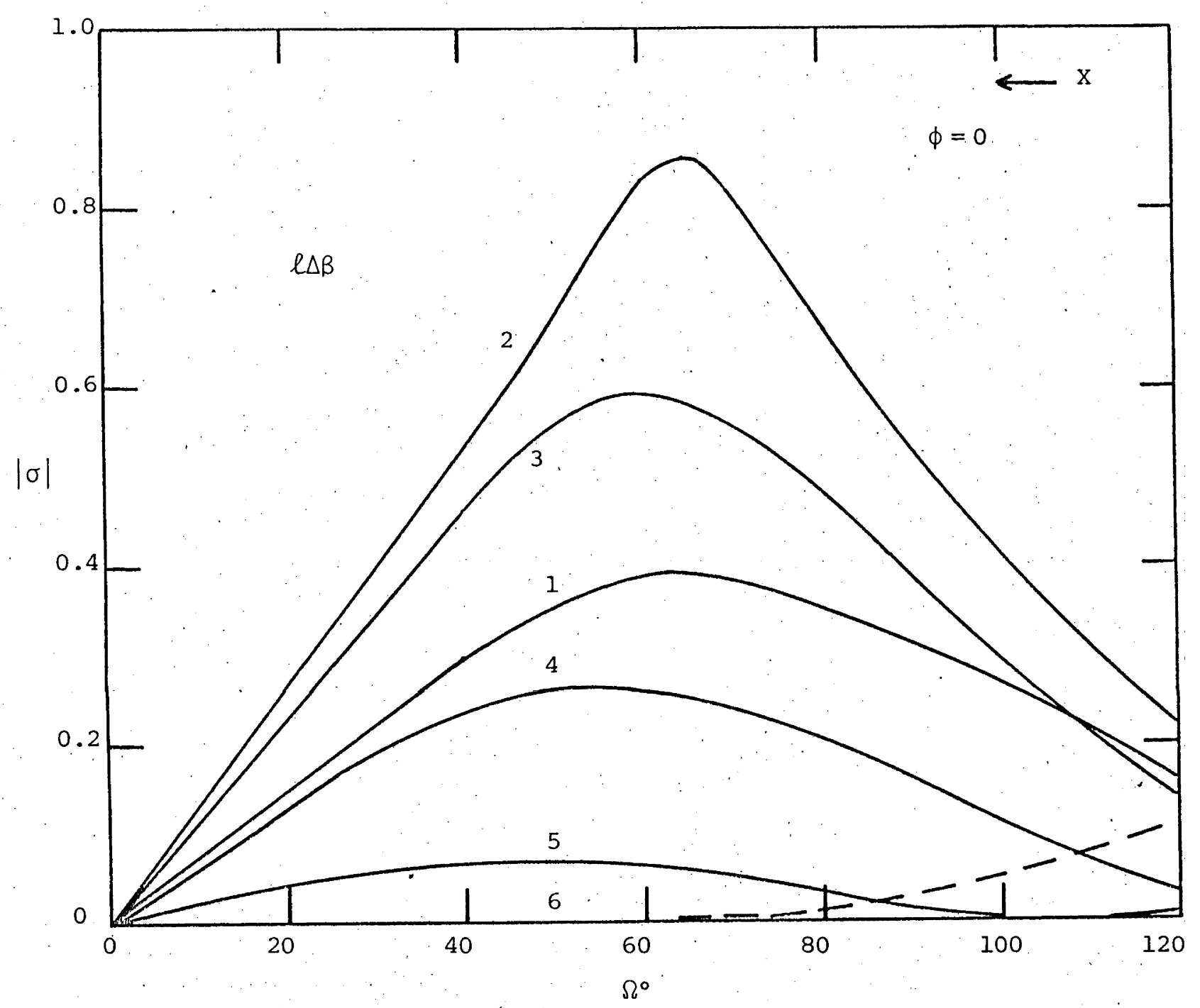

Fig. 8.4. Variation of the ellipticity $\sigma$ with the twist angle $\Omega$, given $\ell \Delta \beta$, i.e. the effect of varying the rate of twist $\xi$. $X$ increases as $\Omega$ decreases. $\sigma$ reaches a maximum value of 1 (circular polarisation) when $\ell \Delta \beta=2.22$ and $\Omega=63.6^{\circ}$ 


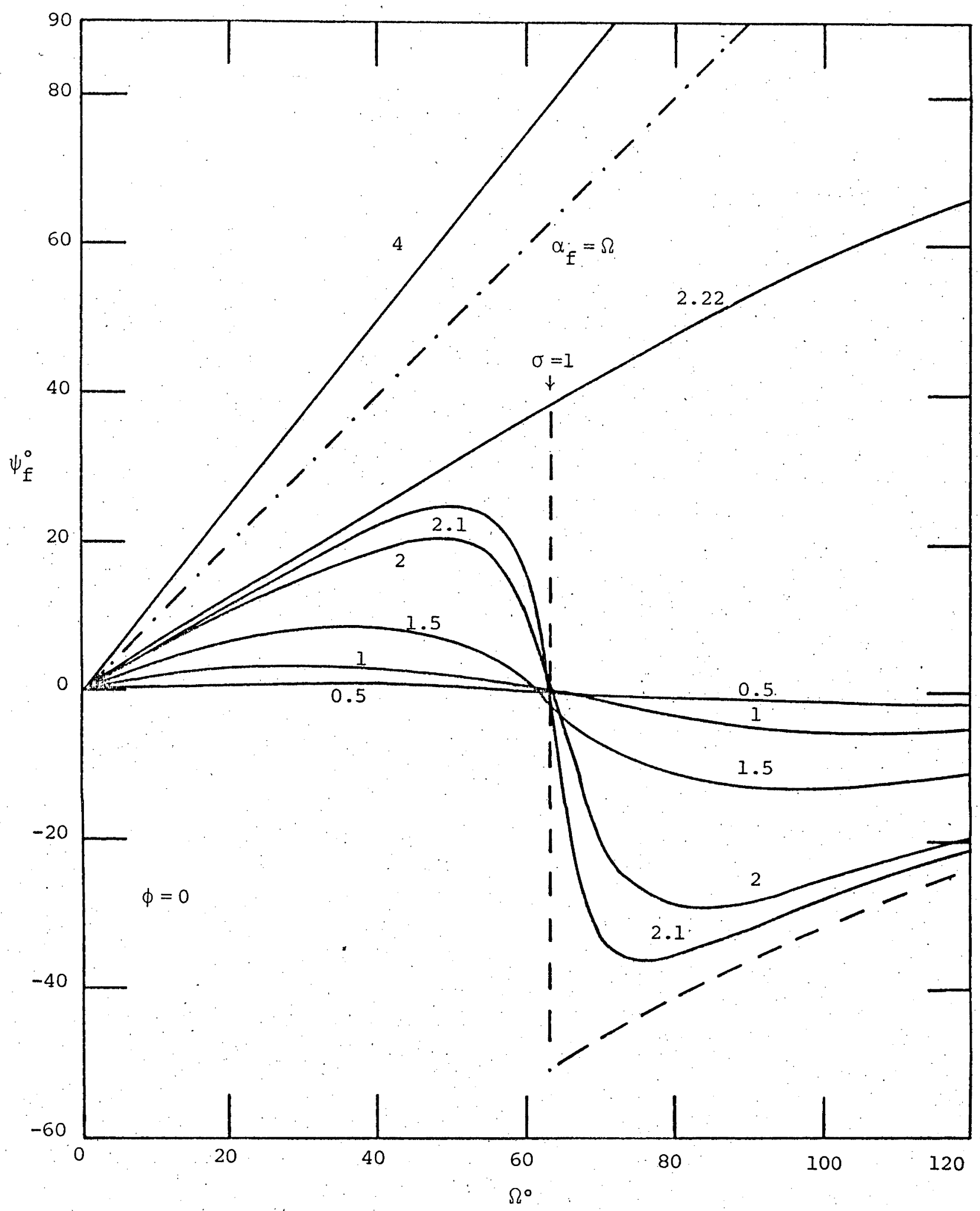

Fig. 8.5. The angle of rotation $\alpha_{f}$ of the polarisation ellipse with respect to the fixed axis $\underline{x}(0)$, as a function of $\Omega$. This shows the effect of varying the twist rate $\xi$ and also demonstrates the discontinuity in $\alpha_{f}$ as the polarisation passes through circular polarisation ( $\sigma=1$ - see text). The line $\alpha_{f}=\Omega(-\longrightarrow \rightarrow)$ is the limit of $\alpha_{f}$ as $x \rightarrow \infty$. 
circular polarisation is also shown. Circular polarisation occurs when $\ell \Delta \beta=2.22$ and $\Omega=63.6^{\circ}$.

In Fig. 8.6 we plot $\sigma$ as a function of $\Omega$, with $\mathrm{x}$ fixed; this shows how the ellipticity varies with position $z$. When $x \leqslant 1, \sigma$ is a slowly varying function of $l$ with a maximum value $\sigma=x$. As $x$ is increased beyond $1, \sigma$ oscillates more rapidly and has a maximum value $1 / \mathrm{X}$. We have

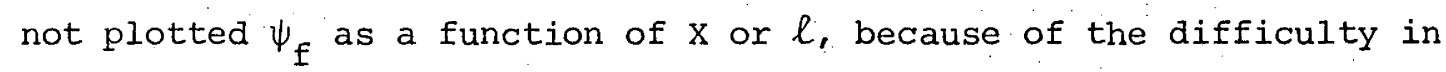
showing the discontinuity.

In the above analysis we have not included the reflected wave which can; under certain circumstances, give rise to significant effects (de Vries, 1951). However in the limit $|\xi| \ll|\beta|$ (the weak-coupling assumption), the reflected field is effectively decoupled from the forward-travelling field and can be treated separately (Chapter 2). Stokes (1963) has shown that, in this limit, de Vries' result reduces to the result in which reflections are neglected.

\subsubsection{Absorption}

Assuming unit initial $(z=0)$ power, the power absorbed by a length $z$ of a twisted medium is given by

$$
P_{A}(z)=1-P(z)
$$

where $P(z)$ is given by (8.22). Substituting (8.11), (8.12) and (8.19) into $(8.22)$ and writing $\xi z / \sqrt{F}=\xi_{z}\left(1+\bar{x}^{2}\right)^{\frac{1}{2}}=R+i S, \bar{x}=x+i Y$ where $\mathrm{X}=\frac{\beta_{\mathrm{x}}-\beta_{\mathrm{y}}}{2 \xi}, \mathrm{Y}=\frac{\alpha_{\mathrm{x}}-\alpha_{\mathrm{y}}}{4 \xi}$ and $\alpha_{\mathrm{s}}=\frac{1}{2}\left(\alpha_{\mathrm{x}}+\alpha_{\mathrm{y}}\right)$, we have

$$
P(z)=\left\{\cos ^{2} R \cosh ^{2} S+\sin ^{2} R \sinh ^{2} S\right.
$$

$+|F|\left[I+X^{2}+Y^{2}-4 Y \operatorname{Re}\left(p q^{*}\right)\right]\left[\sin ^{2} R \cosh ^{2} S+\cos ^{2} R \sinh ^{2} S\right]$

$\left.-\left(p^{*}-q^{*}\right)\{(Y \operatorname{Re} \sqrt{F}+X \operatorname{Im} \sqrt{F}) \sin 2 R+(X \operatorname{Re} \sqrt{F}-Y \operatorname{Im} \sqrt{F}) \sinh 2 S]\right\} e^{-\alpha} s^{z}$ 


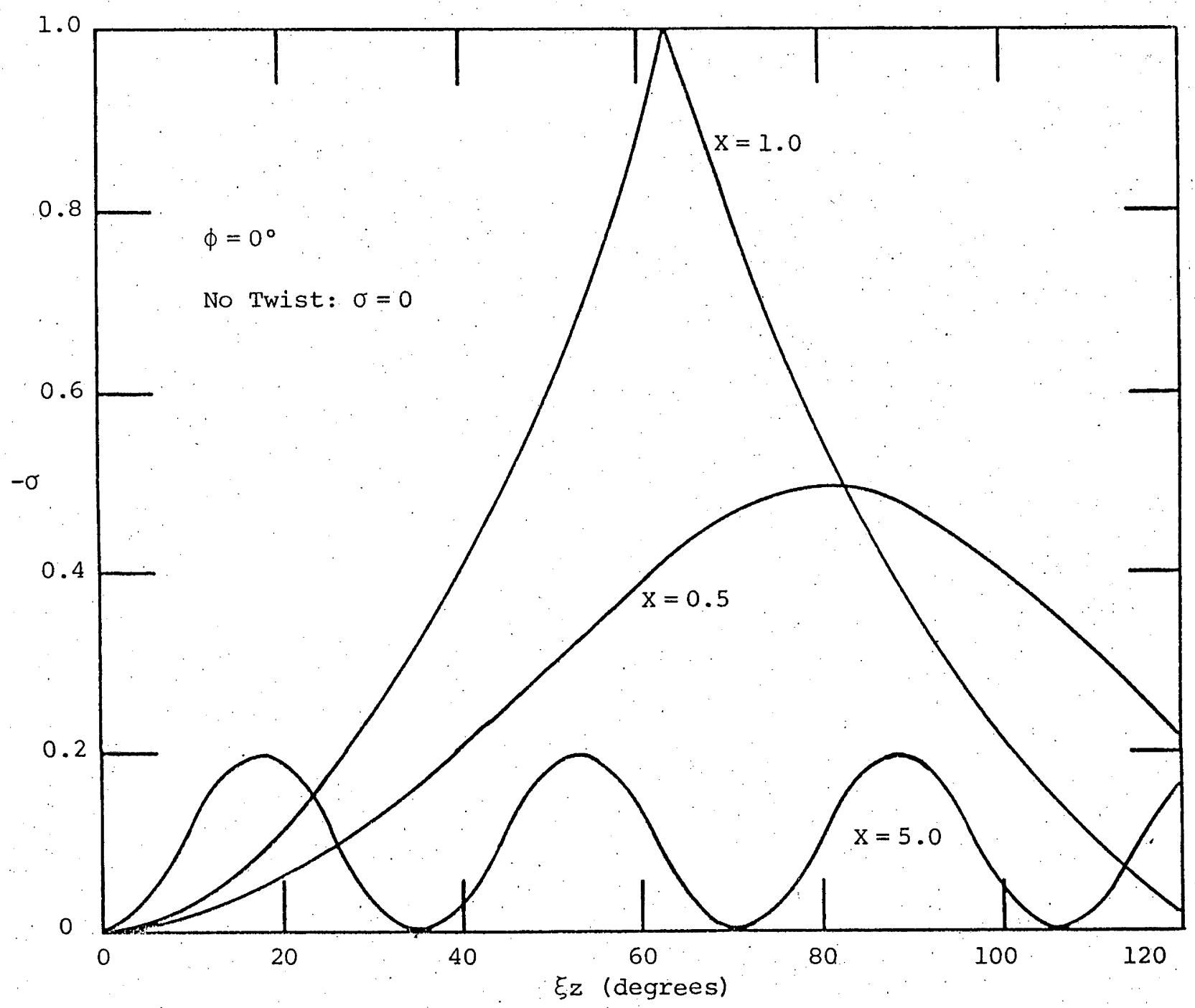

Fig. 8.6. Variation of the ellipticity $\sigma$ with distance $z$ through the medium. Fixed $\mathrm{X}$ means fixed $\Delta \beta$ and fixed $\xi$. When $x \leqslant 1,|\sigma|$ has a maximum value of $x$, whereas for $x \geqslant 1$ the maximum value is $1 / \mathrm{X}$. 
An important example is the absorption of linearly polarised incident light for which $p=\sqrt{2} \cos \phi, q=\sqrt{2} \sin \phi$, where $\phi$ is the angle between the direction of polarisation and the $x(0)$ axis (see section 8.6.2) . The directions for maximum and minimum absorption are given by the stationary values of $(8.37)$ with respect to $\phi$, i.e. $\phi_{\max } \phi_{\min }$ satisfy

$$
\tan 2 \phi=\frac{\mathrm{M}}{\mathrm{N}}
$$

where

$$
\begin{aligned}
& M=2|F| Y\left[\sin ^{2} R \cosh ^{2} S+\cos ^{2} R \sinh ^{2} S\right] \\
& N=(Y \operatorname{Re} \sqrt{F}+X \operatorname{Im} \sqrt{F}) \sin 2 R+(X \operatorname{Re} \sqrt{F}-Y \operatorname{Im} \sqrt{F}) \sinh 2 S
\end{aligned}
$$

The values of $\phi$ for maximum and minimum power absorbed are therefore separated by $90^{\circ}$, as in the non-twisted case. The amount of light absorbed when the incident light is unpolarised is found by averaging (8.37) (with $\mathrm{p}$ and $\mathrm{q}$ given by the linearly polarised values) over $\phi$, giving

$$
\begin{aligned}
& \mathrm{P}_{A}^{\mathrm{UP}}(z)=\left\{\cos ^{2} R \cosh ^{2} \mathrm{~S}+\sin ^{2} R \sinh ^{2} \mathrm{~S}\right. \\
& \left.+|\mathrm{F}|\left[1+\mathrm{X}^{2}+\mathrm{Y}^{2}\right]\left[\sin ^{2} \mathrm{R} \cosh ^{2} \mathrm{~S}+\cos ^{2} R \sinh ^{2} \mathrm{~S}\right]\right\} \mathrm{e}^{-\alpha \mathrm{s}^{z}} \\
& =\frac{1}{2}\left[\mathrm{P}_{\mathrm{A}}^{\max }(\mathrm{z})+\mathrm{P}_{\mathrm{A}}^{\min }(\mathrm{z})\right]
\end{aligned}
$$

In the limit of zero twist, the expression for the power absorbed reduces to (Snyder, 1973)

$$
P_{A}(z)=1-\left[e^{-\alpha x^{z}} \cos ^{2} \phi+e^{-\alpha} z \sin ^{2} \phi\right]
$$

whereas for a large twist rate $(|\bar{x}| \ll 1)$, for which there is also no rotation of the field, 
8.5

$$
\mathrm{P}_{A}(\mathrm{z}) \approx 1-e^{-\left(\frac{\alpha+\alpha}{2}\right) z}
$$

independent of $\phi$. If the incident light is unpolarised, $\cos ^{2} \phi=\sin ^{2} \phi=\frac{2}{2}$ in (8.43).

\subsection{THEORY OF BIREFRINGENCE MEASUREMENT}

The linear birefringence of an untwisted medium of thickness $\ell$ can be measured using a crossed-polariser-analyser system (Fig. 8.7) in the following way (Kapany, 1967).

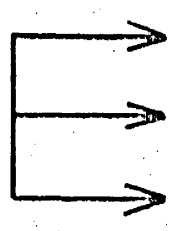

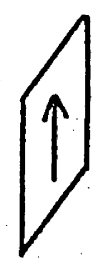

POLARISER

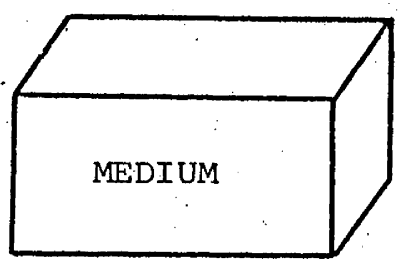

ANALYSER

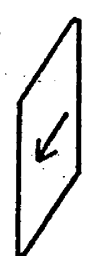

Fig. 8.7

(1) The polariser-analyser is rotated until a minimum intensity (zero in a perfectly linearly birefringent medium) is found at the analyser; this locates one optic axis in the direction of the polariser, and the other at $90^{\circ}$ to it, i.e. in the direction of the analyser.

(2) An incident field consisting of two linearly polarised components with equal amplitudes, one component along each optic axis, is used. The phase difference between the two components is adjusted to give a minimum at the analyser. This phase difference is equal to 
$\ell \Delta \beta=\frac{2 \pi l}{\lambda} \Delta \mathrm{n}$, where $\Delta \mathrm{n}$ is the birefringence and $\lambda$ is the wavelength in vacuum of the test light.

The physical interpretation of this is straightforward. The modes of an untwisted linearly birefringent medium are plane waves, linearly polarised along either of the optic axes. An incident linearly polarised field along one of the optic axes excites only one of the modes, which is polarised at right angles to the analyser, therefore giving zero intensity. In the second part of the experiment both modes are excited and the phase difference between them is adjusted so that the field at the analyser is linearly polarised, again at right angles to the analyser. The measured phase difference is equal to $\ell \Delta \beta$.

The method can also be used if the medium is twisted, but in this case the relationship between the actual linear birefringence and the phase difference found for minimum analyser intensity is much more complicated. In a twisted medium there are in general no true optic axes, and the measured birefringence is a function of both the actual birefringence and the twist angle $\Omega$. We now proceed to find this relationship.

(1) We choose axes as in Fig. 8.1 and apply a linearly polarised incident field at angle $\phi$ to $\underline{x}(0)$. The intensity at the analyser is given by

$$
I=|\underline{E}(\ell) \cdot \underline{r}|^{2}
$$

where

$$
\underline{r} \underline{L}=-\sin \phi \underline{x}(0)+\cos \phi \underline{y}(0)
$$

is a unit vector in the direction of the analyser. Substituting for $\underline{E}(\ell)$ from (8.10), and using (8.23), (8.25), $\Omega=\xi \ell$ and $\Psi=\Omega-\phi$, we find 


$$
I=\frac{1}{2}\left\{\left|A_{x}\right|^{2}+\left|A_{y}\right|^{2}\right\}+\sin 2 \Psi \cdot \operatorname{Re}\left(A_{x} A_{y}^{*}\right)-\frac{1}{2} \cos 2 \Psi\left\{\left|A_{x}\right|^{2}-\left|A_{y}\right|^{2}\right\}
$$

We consider only the lossless case, for which $\frac{1}{2}\left[\left|A_{x}\right|^{2}+\left|A_{y}\right|^{2}\right]=1$. our results apply also for uniform loss $\left(\alpha_{x}=\alpha_{y}\right)$, but not to strongly dichroic media. Extension to this case is straightforward. Then

$$
I=I+\sin 2 \Psi \operatorname{Re}\left(A_{x} A_{y}^{*}\right)-\cos 2 \Psi\left(\left|A_{x}\right|^{2}-1\right)
$$

Substitution of the expressions (8.11), (8.12) with $p=\sqrt{2} \cos \phi$, $q=\sqrt{2} \sin \phi$ into $(8.48)$ and minimizing with respect to $\phi$, we find a minimum of intensity at the analyser at

$$
\phi_{\min }=\Omega / 2
$$

The minimum intensity is non-zero in general, so that $\phi=\Omega / 2$ gives a pseudo optic-axis only.

(2) We now apply an incident field

$$
\underline{E}(0)=e^{-i \ell \Delta \gamma} \cos \phi \underline{x}(0)+\sin \phi \underline{y}(0)
$$

with $\phi=\frac{\Omega}{2}+45^{\circ}$, and minimize (8.48) with respect to $\ell \Delta \gamma$. Putting $p=\sqrt{2} e^{-i \ell \Delta \gamma} \cos \phi, q=\sqrt{2} \sin \phi$ into (8.11), (8.12), calculating (8.48) and minimizing leads to the expression for $\ell \Delta \gamma$ for which $I$ is a minimum or maximum,

$$
\tan (\ell \Delta \gamma)=2 \times \sqrt{F} \sin (\Omega / \sqrt{F})\left[\frac{\cos (\Omega / \sqrt{F}) \cos \Omega+\sqrt{F} \sin (\Omega / \sqrt{F}) \sin \Omega}{\cos (2 \Omega / \sqrt{F}) \cos \Omega+\sqrt{F} \sin (2 \Omega / \sqrt{F}) \sin \Omega}\right]
$$

In the limit of no twist $(x \rightarrow \infty),(8.51)$ reduces to $\tan (\ell \Delta \gamma)=\tan (\ell \Delta \beta)$, as required. The measured birefringence given by (8.51) is a complicated function of $\ell \Delta \beta$ and $\Omega$, because the ellipticity of the field at the analyser and the angle between the major axis of the polarisation ellipse and the analyser direction vary with both these parameters. 
This is a result of there being no optic axes in the twisted medium.

There is an unresolved problem associated with (8.5I), involving the interpretation of the maxima and minima of the intensity at the analyser. The analyser intensity is given by an expression of the form

$$
I=I_{0}+\frac{1}{2} \cos \Omega[A \cos \ell \Delta \gamma+B \sin \ell \Delta \gamma]
$$

where $I_{0}$. A and $B$ are functions of $\Omega$, but are independent of $\ell \Delta \gamma$. We choose $\ell \Delta \gamma$ to minimize (8.52), i.e. to give

$$
I=I_{0}-\frac{1}{2}|\cos \Omega| \sqrt{A^{2}+B^{2}}
$$

However, $\cos \Omega$ changes sign as $\Omega$ passes through $90^{\circ}$, so that there must be a corresponding jump of $\pi$ in the value of $\ell \Delta \gamma$ in order to keep I a minimum. Therefore when $\Omega<90^{\circ}$, the expression in square brackets in (8.52) must be minimized, whereas when $\Omega>90^{\circ}$ (and $\Omega<270^{\circ}$ ), it must be maximized. The values of $\ell \Delta \gamma$ on either side of $\Omega=90^{\circ}$ are solutions of (8.51) because the $\tan ^{-1}$ function is only defined to within $\pi$. The reasons for believing the jump of $\pi$ to be incorrect are based mainly on physical and intuitive grounds. Kirschfeld and Snyder (1975) measure the birefringence of fly rhabdomeres and, because they find $\Delta \gamma$ varies with length, suggest that the rhabdomeres are twisted. However in their results for $\Delta \gamma$ (for lengths of rhabdomere that should give both $\Omega<90^{\circ}$ and $\Omega>90^{\circ}$ for moderate twist rates) there are no discontinuities. This supports my belief that the discontinuity arises from difficulties in interpreting the theory although, in spite of careful checking, I have been unable to discover any inconsistencies except for the discontinuity. Consequently in section 8.6.3, where results are given, the presence of the discontinuity is indicated by dashed Iines. 


\subsection{APPLICATION TO TWISTED INVERTEBRATE PHOTORECEPTORS}

Many invertebrate photoreceptors are sensitive to the direction of polarisation of light (see reviews in Snyder and Menzel, 1975). In general more light is absorbed when the incident light is polarised parallel to the microvilli axis than when it is polarised perpendicular, owing to the dichroic properties of the microvilli.* The polarisation sensitivity (PS) of a retinula cell depends on the arrangement of the microvilli in the rhabdom, on the absorption coefficient $\alpha$ of the microvilli and on electrical coupling (Snyder, 1973; Snyder et al, 1973). In the absence of electrical coupling, $\mathrm{PS} \propto \frac{\mathrm{P}_{\max }}{\mathrm{P}_{\min }}$, where $\mathrm{P}_{\max }$ and $\mathrm{P}_{\min }$ are the maximum and minimum power absorbed, found by measuring the power absorbed as a function of the direction of polarisation of the incident light. The dichroic ratio $\Delta=\frac{\alpha}{\alpha}{ }_{\perp}$, where $\alpha_{\|}, \alpha_{\perp}$ are the absorption coefficients of the microvilli for light polarised parallel and perpendicular respectively, to the axis of the microvilli. $\Delta$ depends on the arrangement and packing of the microvillar membrane (form dichroism), as well as on the orientation and dichroic properties of the chromophores associated with the membrane (intrinsic dichroism) (see Israelachvili et al, 1975; Gribakin and Govardovskii, 1975; Laughlin et al, 1975; Schlecht and Täuber, 1975; Snyder and Laughlin, 1975). If the absorbing dipoles are highly aligned within the microvilli, $\Delta$ can be as large as 20 , whereas for random alignment $\Delta=1.67$. Snyder and Laughlin (1975) show that, in symmetric fused rhabdoms, the absorbing dipoles should be highly aligned to maximise absorption. A consequence of this alignment is a high dichroic ratio and therefore a high theoretical PS of individual rhabdomeres (Snyder, 1973). High values for the PS of rhabdomeres in ant and worker bee

\footnotetext{
* An exception is rhabdomere 7 of fiy.
} 
have indeed been measured, but in many cases, the measured PS is very much lower than expected (Snyder et al, 1973; Menzel, 1975). One explanation for this discrepancy is that electrical coupling between retinula cells reduces the PS; experimental evidence for electrical coupling has been found in the dragonfly rhabdom (Snyder et al, 1973). A second possible PS-reducing mechanism is that of twisting of the rhabdom. A high PS depends on accurate alignment of the microvilli along the length of the rhabdom; so that any twisting, which destroys this alignment, will reduce the PS. Twisting has been observed in the bee rhabdom by Grundler (1974) and measured in the ant rhabdom by Menzel (1975) and Menzel and Blakers (1975). The effect of twisting on the PS of retinula cells in a fused rhabdom is investigated in Section 8.6.1.

Polarisation detection in the bee and ant is probably carried out by the ninth cell (Menzel and Snyder, 1974; Menzel, 1975). The rhabdomere of the ninth cell is only present in the proximal third of the rhabdom and lies underneath one of the other rhabdomeres, which acts as a polarisation filter. However if the rhabdom twists, the polarisation-filter effect is reduced and consequently, the PS of the ninth cell is decreased. This is also examined in Section 8:6.1. The measured PS of rhabdomeres $1-6$ of $f l y$ is low (PS $\sim 2$ ) (Kirschfeld, 1969). This is consistent with the prediction of snyder and Laughlin (1975) that, for maximum absorption of unpolarised light in fly-type rhabdomeres, the absorbing dipoles should be randomly aligned within the microvilli, giving $\Delta \approx 2$. An alternative scheme is for the microvilli in the fly rhabdomeres to have a high $\Delta$ (alignment of dipoles) and for the rhabdomeres to twist. Fly rhabdomeres differ from the 
symmetric fused rhabdom of section 8.6.1, in that they are birefringent and aichroic. The PS and the absorption of unpolarised light in twisted fly rhabdomeres are examined in section 8.6.2.

The birefringence of rhabdomeres is an important measure of the anisotropy of the microvilli structure, and has also been used to determine the waveguide parameter V (Kirschfeld and snyder, 1975). However when the rhabdomere is twisted, the measured birefringence becomes a complicated function of the actual birefringence and of the twist angle. This may explain the variation of the birefringence with length as found by Kirschfeld and' Snyder (1975). In Section 8.6.3 we give an example, using the theory of section 8.5, of how the measured birefringence of a fly rhabdomere is affected by the twist.

8.6.1 Absorption of Light in Twisted Fused Rhabdoms

For simplicity we assume that the rhabdom has a symmetrical arrangement of microvilli as shown in Fig. 8.8, and that each rhabdomere

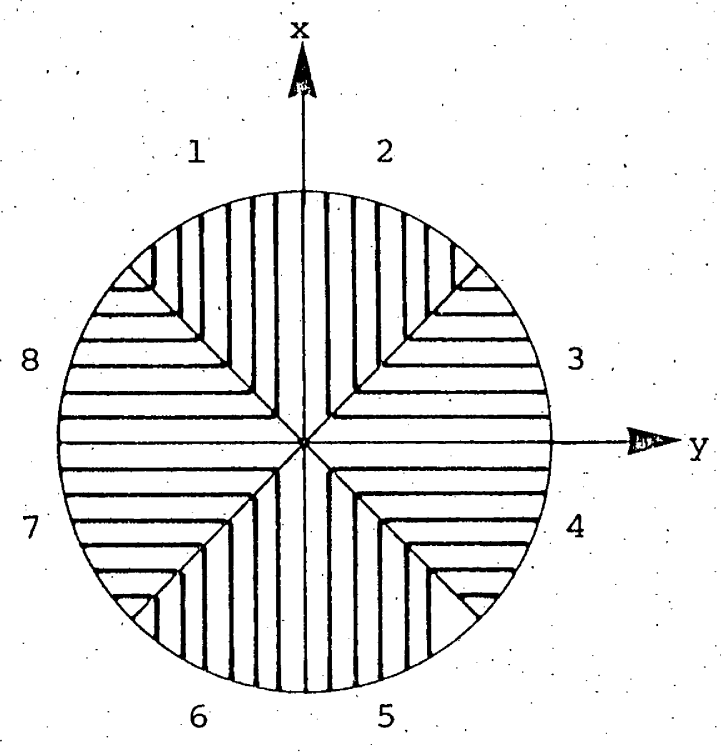

Fig. 8.8 
has the same area in cross-section and the same absorption coefficient. These assumptions display the physics of twisting without a significant loss of generality. The effect of departures fxom this symmetry is discussed later in this section.

The symmetric structure of Fig. 8.8 has zero total birefringence and zero total dichroism $(\Delta \beta=\Delta \alpha=0)$, so that there is no rotation of the field direction with the twist. This greatly simplifies the mathematical formulation. The absorption of the rhabdom as a whole is given by

$$
P_{A}(\ell)=1-e^{-\left(\frac{\alpha \|^{+\alpha}}{2}\right) l}
$$

where $\alpha_{\|}, \alpha_{\perp}$ are the microvilli absorption coefficients when the electric vector is respectively parallel and perpendicular to the axis of the microvilli, and $l$ is the length of the rhabdom. The result (8.54) is valid for polarised and unpolarised light. We conclude that the absolute sensitivity of a symmetric fused rhabdom, taken as a whole, is unaffected by twisting.

\section{Absorption by Individual Rhabdomeres}

The amount of power $\mathrm{P}_{A}^{i}$ absorbed by rhabdomere $i$ is given by

$$
\mathrm{P}_{\mathrm{A}}^{\mathrm{i}}=\mathrm{P}^{i} \|^{\mathrm{i}}+\mathrm{P}
$$

where

$$
\begin{aligned}
& P^{i} \|=\frac{\alpha}{A} \int_{0}^{A_{i}} e^{\ell-\left(\frac{\alpha U^{+\alpha}}{2}\right)} z \cos ^{2} \Psi(z) d z \\
& P^{i}=\frac{\alpha \perp^{A} i}{A} \int_{0}^{l} e^{-\left(\frac{\perp^{+\alpha} \perp}{2}\right) z} \sin ^{2} \Psi(z) d z
\end{aligned}
$$


and

$$
\Psi(z)=\Omega(z)-\phi
$$

$A, A_{i}$ are respectively the cross-sectional areas of the rhabdom and of rhabdomere $i, \phi$ is the angle between the direction of polarisation (DP) of the incident field and the axis of the microvilli of rhabdomere $i$, and $\Omega(z)$ is the twist angle at position $z$.

If the twist is uniform, $\Omega(z)=\xi z$ where $\xi$ (the rate of twist) is a constant. In this case the integrals in (8.56), (8.57) are known, giving the power absorbed in rhabdomere $i$ as

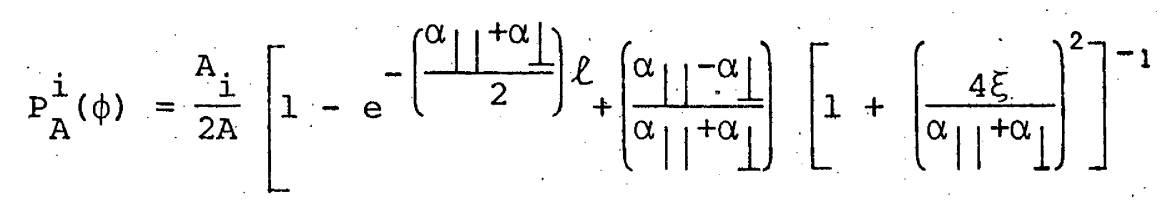

$$
\begin{aligned}
& \times\left\{e^{-\left(\frac{L^{+\alpha}}{2}\right)}\right)\left[\left(\frac{4 \xi}{\alpha||^{+\alpha} \mid}\right) \sin 2(\Omega-\phi)-\cos 2(\Omega-\phi)\right] \\
& \left.\left.+\left(\frac{4 \xi}{\alpha \|^{+\alpha} 1}\right) \sin 2 \phi+\cos 2 \phi\right\}\right]
\end{aligned}
$$

If the incident light is unpolarised, an average over $\phi$ in (8.59) gives

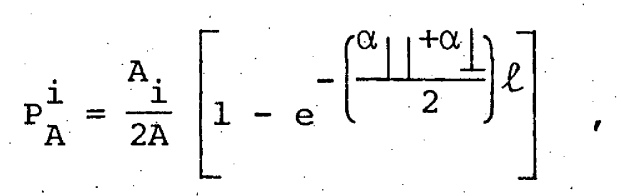

which is the same result as for untwisted fused rhabdoms (Snyder, 1973). Therefore the absolute sensitivity of individual rhabdomeres to unpolarised light is unaffected by twisting.

Maximum and minimum absorption of polarised light by an individual rhabdomere occurs when $\phi$ is given by 


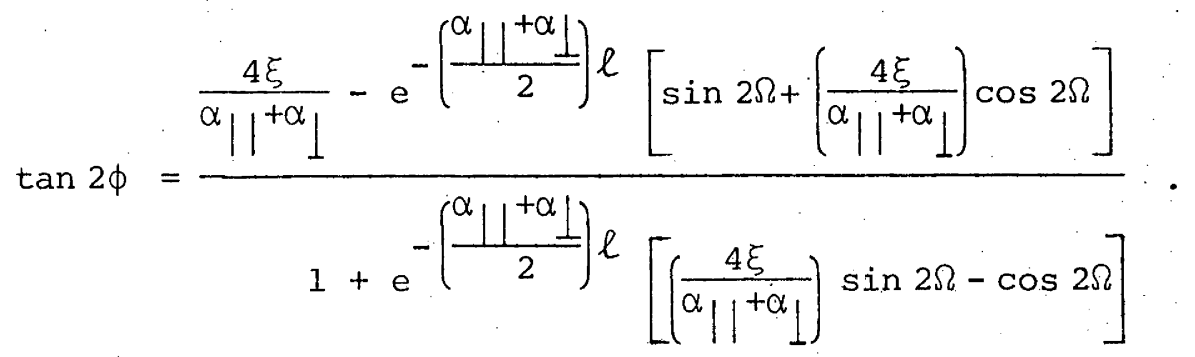

In Fig. 8.9 we show $\phi$ for maximum absorption as a function of total twist angle $\Omega$, for several values of $\alpha_{\max } l$ and dichroic ratio $\Delta=\alpha_{1} / \alpha \perp^{\text {. }}$ If the absorption per unit length is small, $\phi_{\max } \approx \Omega / 2$, as is expected from the symmetry of the twisted fused rhabdom. As the absorption is increased, a greater proportion of the absorption takes place in the distal part of the rhabdomere, so that the effective twist seen by the light is smaller and $\phi_{\max }$ decreases. If there is no twist, $\phi_{\max }=0$ (E is parallel to the microvilli) in a symmetric fused rhabdom. If the rhabdom is not symmetric, $\phi_{\max } \neq 0$ even when the rhabdom is not twisted (Snyder and Sammut, 1973, Section 8.6.2).

The polarisation sensitivity (PS) of an individual retinula cell in the absence of electrical coupling is given by (Shaw, 1969, 1969a; Snydex, 1973)

$$
\operatorname{PS}=\mathrm{P}_{\mathrm{A}}^{\mathrm{i}}\left(\phi_{\max }\right) / \mathrm{P}_{\mathrm{A}}^{i}\left(\phi_{\min }\right)
$$

where, from (8.61), $\phi_{\min }=\phi_{\max } \pm 90^{\circ}$. The PS as a function of $\Omega$ is illustrated in Fig. 8.10, which shows that twisting reduces the PS. The greater the dichroic ratio, the greater is the percentage reduction in the PS. The PS reduction is relatively insensitive to the amount of light absorbed, i.e. to $\left.\alpha\right|^{l}$.

Menzel (1975) and Menzel and Blakers (1975) find that the rate of twist in the bulldog ant Myrmecia gulosa is approximately constant 


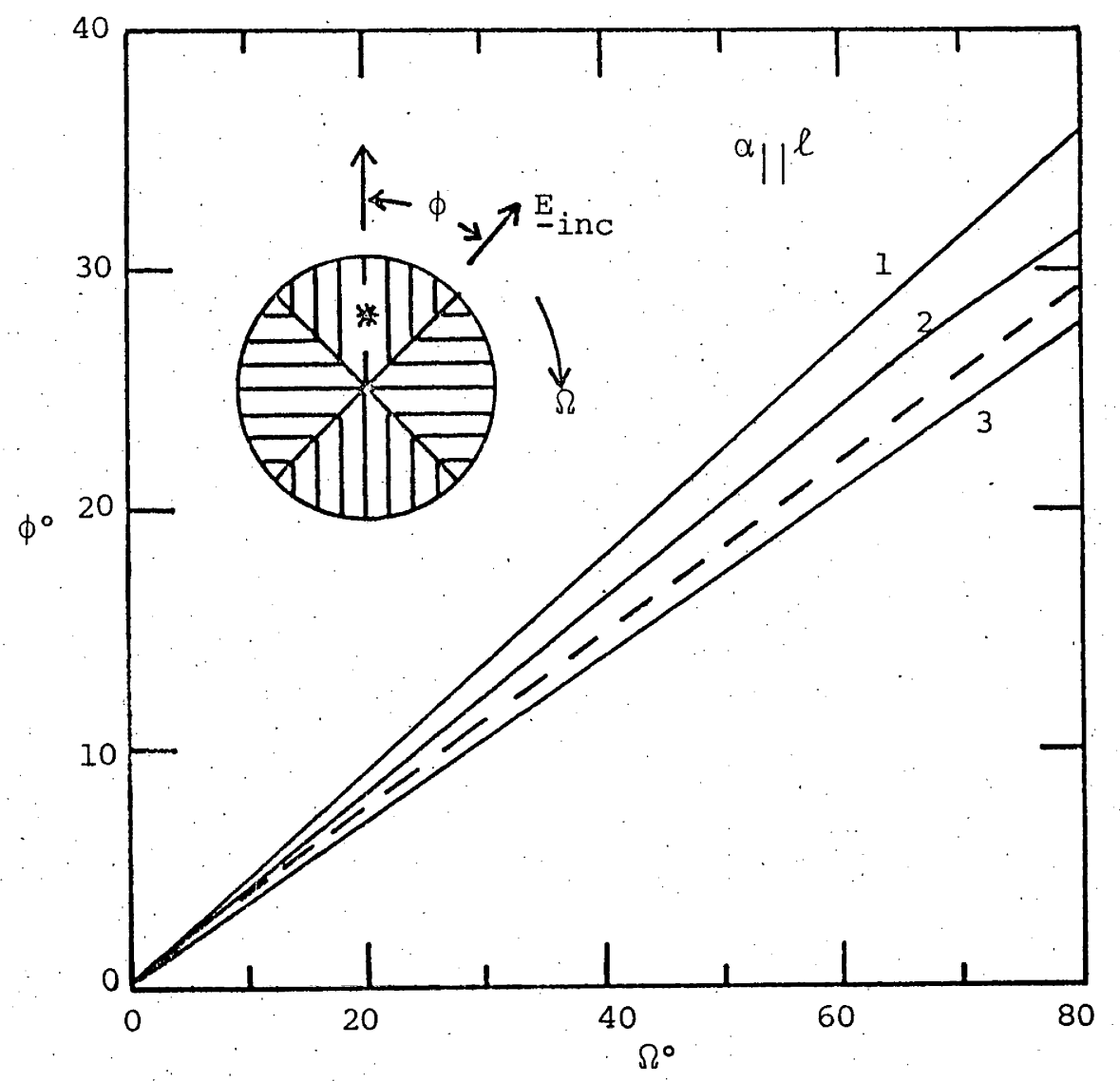

Fig. 8.9. The angle $\phi$ for maximum absorption of

- incident linearly polarised light by either of the two rhabdomeres in the quadrant marked *, as a function of the total twist angle $\Omega . \Delta=10$ except for the dashed line for which $\left.\alpha\right|^{l=2}$ and $\Delta=2$. 


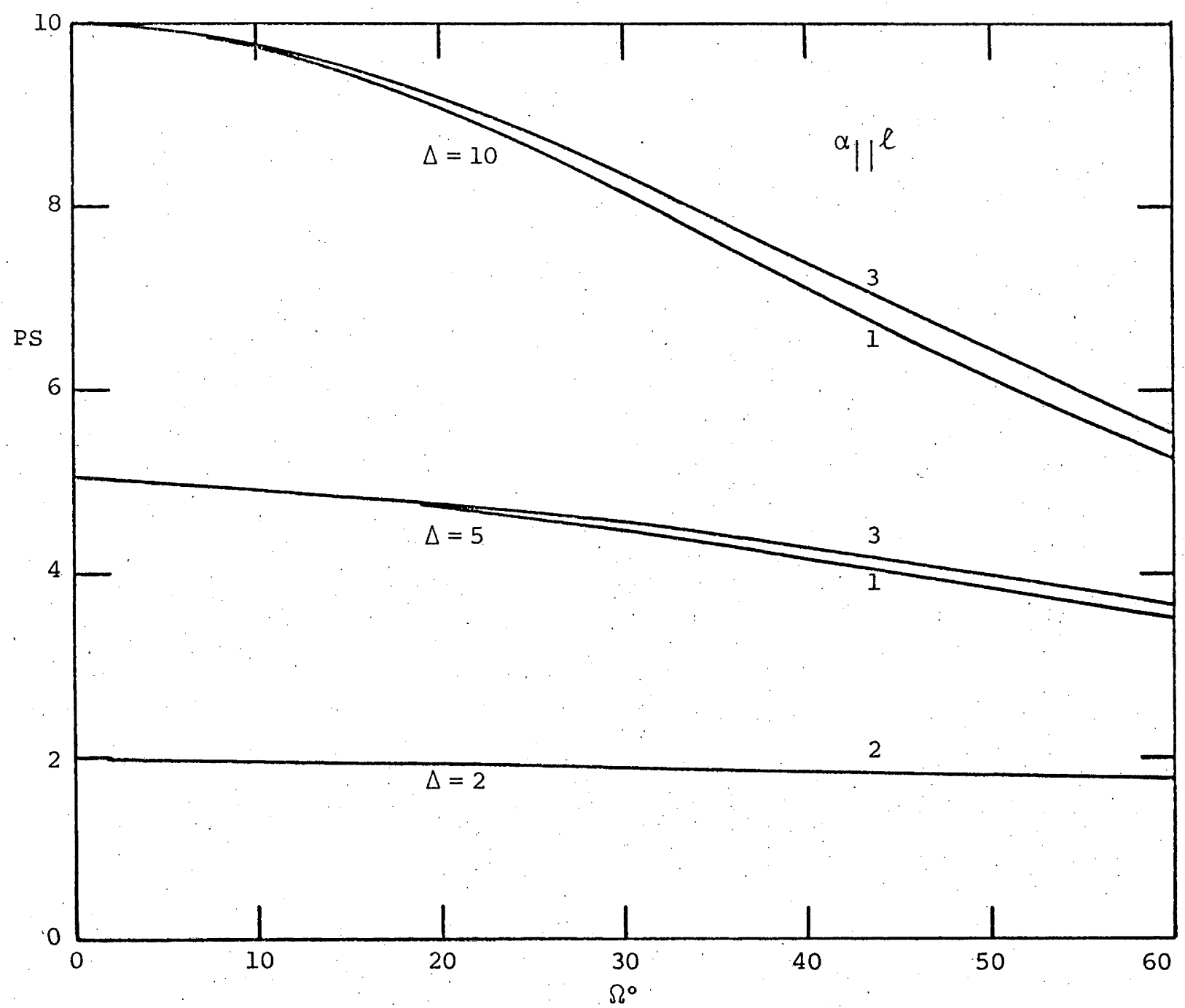

Fig. 8.10. The polarisation sensitivity of an individual retinula cell. in a symmetric fused rhabdom (Fig. 8.8), as a function of the total twist angle $\Omega . \Delta$ is the dichroic ratio and $\alpha / \mid$ is the absorption coefficient for light polarised parallel to the microvilli axis. The total power absorbed by the rhabdom is

\begin{tabular}{ccc}
$\Delta$ & $\alpha H^{l}$ & $\mathrm{P}_{\mathrm{A}}(8)$ \\
\hline 10 & 3 & 80.8 \\
5 & 1 & 42.3 \\
& 3 & 83.5 \\
2 & 1 & 45.1 \\
& 2 & 77.7
\end{tabular}

Waveguide effects are not included i.e. $n=1$. 
over the first 90-100 $\mu$ of the rhabdom, and that it flattens out to approximately zero for the remaining length. The average total twist in $100 \mu$ is about $60^{\circ}$.

We first need to determine whether the ant rhabdom is sufficiently symmetric for the purposes of our theory. If there is only a small overall birefringence and dichroism, our assumption of zero rotation of the direction of polarisation with the twist is valid, and the results of Figs. 8.9, 8.10, can be applied to the ant rhabdom. The arrangement of the microvilli directions in the ant rhabdom is. symmetric, although the symmetry differs from that of Fig. 8.8 (Menzel, 1972). Therefore we might expect that the overall birefringence of the rhabdom is small. This remains to be verified experimentally. The symmetry of the rhabdom as regards absorption is broken by the presence of two uv (absorbing) rhabdomeres with parallel microvilli, among six green rhabdomeres (Menzel, 1972). Therefore it is possible, especially at uv wavelengths at which only the uv cells are absorbing, that the rhabdom as a whole is dichroic. The relevant quantity for use in the theory of Section 8.3 is $\Delta \xi^{\mathrm{A}}=\xi_{\mid}^{\mathrm{A}}-\xi^{\mathrm{A}}$; $\|$, $\perp$ here indicate two axes parallel and perpendicular to the microvilli axis of the uv cells, and $\xi^{\mathrm{A}}$ is defined as (Snyder, 1973)*

$$
\xi^{A}=\frac{1}{A} \sum_{j=1}^{\&} A_{j} \alpha_{j}
$$

$\alpha_{j}$ is the absorption coefficient of rhabdomere $j$ and we are considering the distal two-thirds of the rhabdom in which there are eight rhabdomeres. For no rotation of the DP we require $\frac{\ell \Delta \xi^{A}}{4 \Omega} \ll 1$ (Section 8.4 ), which for

\footnotetext{
${ }^{*}$ We use the superscript $A$ to distinguish the absorption coefficient $\xi^{A}$ from the rate of twist $\xi$. A, $A_{j}$ are the cross-sectional areas of the rhabdom and rhabdomere $j$ respectively.
} 
a twist $\Omega=60^{\circ} \approx 1$ radian, gives $\frac{\ell \Delta \xi^{A}}{4} \ll 1$. It can be seen from Fig. 9 of Menzel (1975) that $\xi_{\|}^{A}=\xi_{1}^{A}$ at $\lambda \approx 400 \mathrm{~nm}$ and we find that the inequality is, in general, satisfied at all wavelengths (see below). Therefore we predict that there is little or no rotation of the DP with the twist in the ant rhabdom.

If we now assume a dichroic ratio of 10 (Menzel, 1975), Fig. 8.10 shows that the PS is reduced from about 10 to about $5-5.5$ when $\Omega=60^{\circ}$. This PS is still higher than that measured for many retinula cells in the ant (Menzel, 1975), which suggests that electrical coupling is also present in these cells. Conversely, in the green cells, for which a high PS has been measured, both electrical coupling and twisting must be absent.

If the overall birefringence and dichroism of the rhabdom are not negligible as we have assumed, there will be some rotation of the DP with the twist (Section 8.4). This will diminish the effect of the twist, so that values for the PS will be higher than given by Fig. 8.10. These curves therefore illustrate the maximum effect the twist can have on the PS. A measurement of the rotation of the DP of light on passing through rhabdom will provide a direct indication of the presence of birefringence and/or dichroism of the rhabdom, irrespective of whether or not the rhabdom is twisted. Such an investigation has been carried out by Täuber (1974) on fly rhabdomeres.

Effect of Twisting on PS of the Ninth Cell of Ant

The ninth retinula ceil in the ant lies beneath one of the two uv cells, and appears at about $90 \mu$ below the distal end of the rhabdom (Menzel, 1972). Menzel and Snyder (1974) and Gribakin (1974) suggest that it is the ninth cell in ant and bee which is the polarisation 
detector, and show theoretically that the PS of the ninth cell is enhanced by the filtering of the light by the rhabdomere directly above it. The maximum polarisation-filter effect occurs when the microvilit of the ninth cell are perpendicular to those of the cell directly above it. If however the top cell is twisted along its length, this enhancement of the PS of cell 9 will be reduced. We now analyse this situation. The ninth cell in the ant and bee is a uv cell, with little. absorption above $450 \mathrm{~nm}$ (Menzel and snyder, 1974; Menzel, 1975). The six green cells absorb very little uv light, so that at uv wavelengths only cells 1 and 5 in the distal two-thirds of the rhabdom, and cells 1 or 5 and 9 in the proximal third, absorb light. Cells 1 and 5 have parallel microvilli, so that there is an overall dichroism. We first show that this dichroism is sufficiently small that there is no rotation of the DP with the twist.

If we assume that cells 1 and 5 each occupy $\frac{1}{8}$ of the total cross-sectional area of the rhabdom, the absorption coefficient for the rhabdom is given by

$$
\xi^{\mathrm{A}}=\frac{\alpha^{\mathrm{uv}}}{4}
$$

Then, the maximum difference in the absorption coefficient is given by

$$
\begin{aligned}
\Delta \xi^{\mathrm{A}} & =\frac{\alpha\rfloor^{\mathrm{uv}}-\alpha \perp^{\mathrm{uv}}}{4} \\
& =\frac{\alpha \coprod_{1}^{\mathrm{uv}}(1-1 / \Delta)}{4}
\end{aligned}
$$

and

$$
\frac{\ell \Delta \xi^{\mathrm{A}}}{4 \Omega}=\frac{\alpha \bigcup^{\mathrm{uv}} \ell}{16 \Omega}(1-1 / \Delta)
$$

For no rotation of the $D P$, we require $\frac{\ell \Delta \xi^{A}}{4 \Omega} \ll 1$, or equivalently 
$F=\left[1+\left(\frac{l \Delta \xi^{\mathrm{A}}}{4 \Omega}\right)^{2}\right]^{-1} \approx 1$ (Section 8.4). In Table 8.1 we give values for these parameters taking $\alpha \|^{\ell} l=2$ and $\Delta=10$ (Menzel, 1975).

\begin{tabular}{|r|c|c|}
\hline$\Omega^{\circ}$ & $\frac{\ell \Delta \xi^{\mathrm{A}}}{4 \Omega}$ & $\mathrm{F}$ \\
\hline 5 & 1.29 & 0.38 \\
10 & 0.64 & 0.71 \\
20 & 0.32 & 0.91 \\
30 & 0.21 & 0.96 \\
40 & 0.16 & 0.97 \\
60 & 0.11 & 0.99 \\
80 & 0.08 & 0.99 \\
\hline
\end{tabular}

We conclude from Table 8.1 that the rotation of the DP is small when $\Omega>20^{\circ}$.

Assuming no rotation of the DP, the power at position $z$ (distal to the ninth cell) is given by

$$
P(z, \phi)=\exp \left\{-\frac{\left.\xi^{A}\right|^{z(1+1 / \Delta)}}{2}-\frac{\xi \|^{z(1-1 / \Delta)}}{2} \frac{\sin 2(\Omega-\phi)}{2 \Omega}\right\},
$$

where $\phi$ is the angle between the microvilli axis of cells 1 and 5 and the direction of polarisation. The microvilli of $I$ and 5 at the distal end of the rhabdom are at $90^{\circ}$ to the microvilli of cell 9 (Menzel and Blakers, 1975), so that if rhabdomere 9 does not twist, $\phi=90^{\circ}, 0^{\circ}$ for maximum and minimum absorption respectively by cell 9 . Therefore (c.f. Menzel and Snyder, 1974)

$$
\begin{aligned}
& \text { PS }(\text { of } 9 \text { th cell })=\exp \left\{\xi^{A}|| \ell(1-1 / \Delta) \frac{\sin 2 \Omega}{2 \Omega}\right\} \text {. } \\
& x \text { PS (of } 9 \text { th cell if it were alone). }
\end{aligned}
$$


$\xi^{A} \mid$ is the absorption coefficient of the distal part of the rhabdom for light polarised parallel to the microvilli axis at the distal end of cells 1 and 5 , and $l$ is the length of rhabdom distal to cell 9. Taking the value $\left.\xi_{\mid}^{A}\right|^{\ell=2}$ (Menzel and Snyder, 1974), we give in Table 8.2 values for the exponential enhancement factor in (8.68).

\begin{tabular}{|c|c|c|}
\hline \multirow{2}{*}{$\Omega^{\circ}$} & \multicolumn{2}{|c|}{ ENHANCEMENT FACTOR } \\
\cline { 2 - 3 } & $\Delta=4$ & $\Delta=10$ \\
\hline 0 & 4.5 & 6.0 \\
20 & 4.0 & 5.3 \\
40 & 2.9 & 3.6 \\
60 & 1.9 & 2.1 \\
80 & 1.2 & 1.3 \\
\hline
\end{tabular}

Table 8.2

Therefore if $\Delta=4$ and the PS of cell 9 above is 3 (Menzel and Snyder, 1974), the PS of cell 9 is 13.5 without twisting, but with a twist of $60^{\circ}, \mathrm{PS}=5.7$. If there is further twisting of the rhabdom at the level of the ninth cell, the PS will be further reduced. The twist at this level does appear to be small however (Menzel and Blakers, 1975).

A further consequence of the twisting of the rhabdom is that the microvilli of whichever of cells 1 and 5 continues throughout the length of the rhabdom will no longer be perpendicular to the microvilli of cell 9. This will cause a reduction in the lateral polarisationfilter effect that this cell has on cell 9 because of optical coupling (Menzel and Snyder, 1974), and leads to a further decrease in the PS of cell 9 .

We conclude that the twisting of the rhabdom plays an important part in the reduction of the PS of the eight long retinula cells. 
A consequence of the twist is a recluction of the PS of retinula cell 9 , but there still remains a sufficient enhancement of the ninth-cell PS, due to the polarisation filtering of the rhabdomere directly above cell 9, to explain the measured values. The twist could also explain why the theoretically predicted high PS for cell 9 is not measured (Menzel and Snyder, 1974).

8.6.2 Absorption of Light in Twisted Fly Rhabdomeres The measured PS of rhabdomeres $1-6$ in $f l y$ is low (PS $\lesssim 2)$, and this has been interpreted as being due to a small dichroic ratio $\Delta \approx 2$ (Kirschfeld, 1969). A low value for $\Delta$ (indicating a random alignment of the absorbing dipoles in the microvilii) has also been predicted by Snyder and Laughlin (1975), for maximum absorption of unpolarised light. However a possible alternative is for the fly rhabdomeres to have a high dichroic ratio and to twist. In this section we examine the PS and the absorption of unpolarised light in a twisted fly-type rhabdomere (Fig. 8.11).
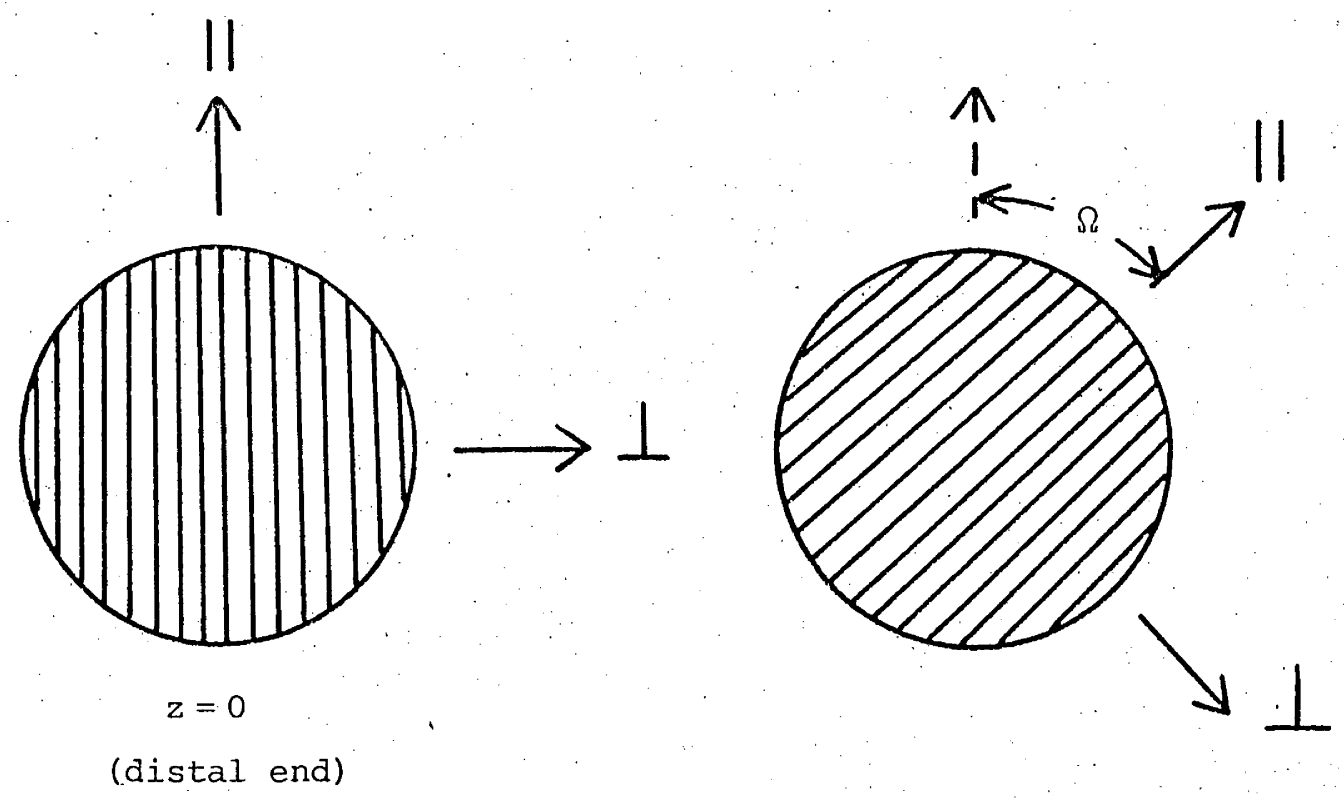

Fig. 8.11 
Because fly rhabdomeres are birefringent and dichroic, the theory for absorption is more complicated than in symmetric fused rhabdoms. Taüber (1974) shows that in an untwisted rhabdomere this anisotropy causes incident linearly polarised light to become elliptically polarised, and the polarisation ellipse to rotate as the light passes along the rhabdomere. The twisting of the rhabdomere can have a similar effect, or can effectively decrease the anisotropy, according to the values of the parameters $\frac{\pi\left(\mathrm{n} \perp-\mathrm{n}||^{\prime}\right.}{\lambda \xi}, \frac{\alpha \perp^{-\alpha} \perp}{4 \xi}$, where $\mathrm{n} \perp^{\prime} \mathrm{n} \|$ are the refractive indexes and $\alpha_{\perp}, \alpha_{\|}$are the absorption coefficients. for light polarised perpendicular and parallel respectively to the microvilli axis, $\xi$ is the rate of twisting and $\lambda$ is the wavelength in vacuum (see Fig. 8.11 and Section 8:4). If these parameters are large, the absorption is much the same as in an untwisted rhabdomere; if they are small, the anisotropy is reduced. An expression for the power abșorbed in a twisted fly-type rhabdomere is given by $(8.36),(8.37)$. The modes of a twisted rhabdomere are in general elliptically polarised, rather than linearly polarised as in the untwisted case.

In Fig. 8.12 we show the PS as a function of twist angle for several values of the birefringence $\Delta \mathrm{n}$ and the dichroic ratio $\Delta$ (the PS is independent of the sign of $\Delta \mathrm{n}$ and $\left.\alpha \|^{-}-\alpha_{1}\right)$. These curves demonstrate the effect of the direction of polarisation (DP) rotating with the twist. For large values of $\Delta n$, the DP approximately follows the twist and, consequently, the PS remains almost constant at its untwisted value (Snyder, 1973). In this case the initial DP for maximum absorption is approximately parallel to the microvilli axis (in fly rhabdomeres 1-6; in rhabdomere 7 the direction for maximum absorption is at right angles to the microvilli axis - Kirschfeld, 1969). As $\Delta \mathrm{n}$ is decreased, the DP 


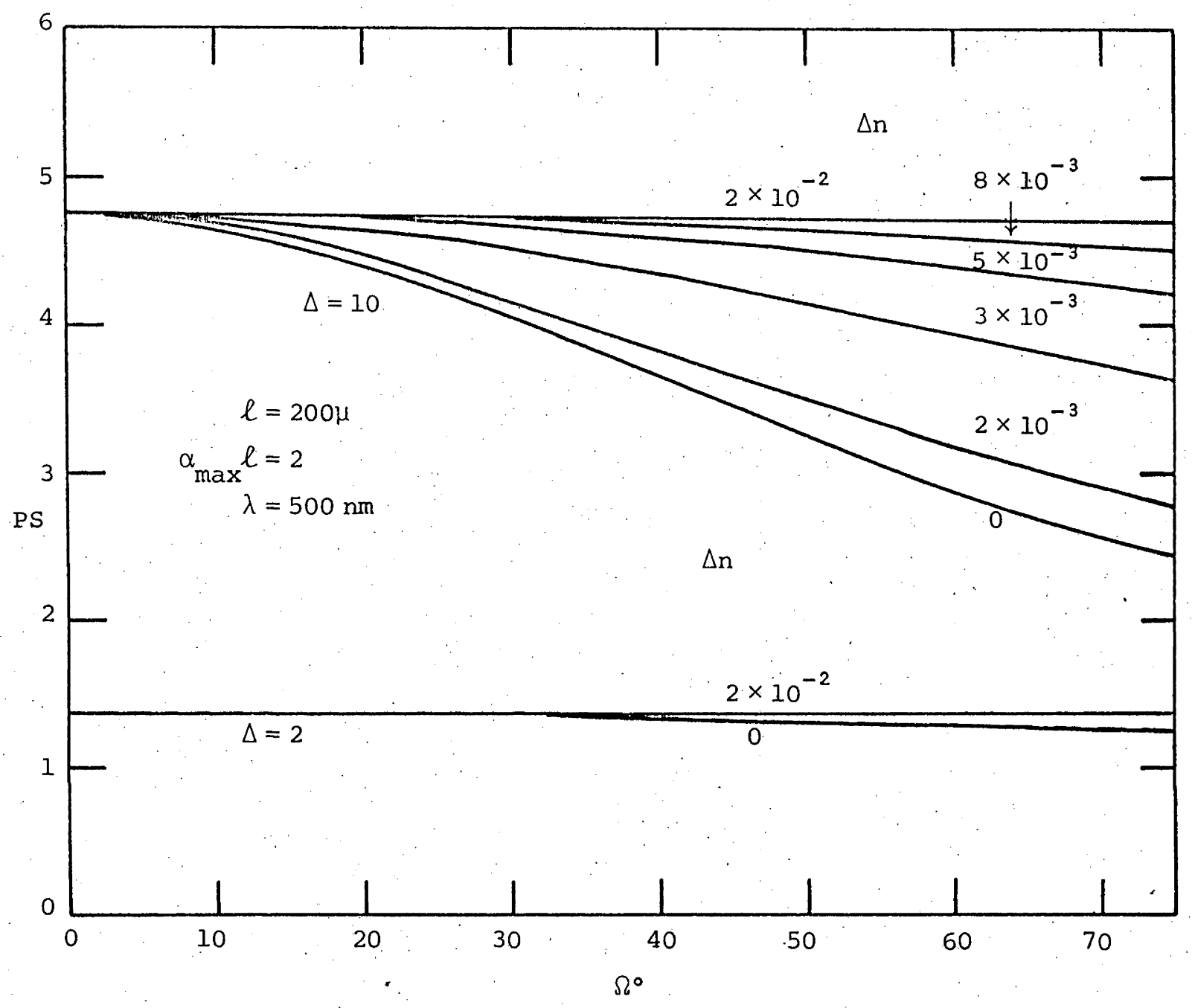

Fig. 8.12. The polarisation sensitivity of a fly-type rhabdomere as a function of total twist angle $\Omega$, for a range of values of the birefringence $\Delta n$. Waveguide effects are not included i.e. $n=1$. 
rotates more slowly than the rhabdomere twists (and oscillates) decreasing the effective dichroism and reducing the PS. The direction for maximum absorption in this case tends to a value of approximately $\Omega / 2(\Omega$ is the total twist angle) as $\Delta \mathrm{n} \rightarrow 0$ (see section 8.6.1). The birefringence measured by Kirschfeld and Snyder (1975) lies in the range $1.2-6.7 \times 10^{-3}$, so that the average lies about midway between the two limiting cases in Fig. 8.12

The percentage reduction in the PS because of the twist is greatest for large $\Delta$, as can be seen in.Fig. 8.13. This shows that a twist of $70^{\circ}$ can reduce the PS from 5 (taking $\Delta=10$ ) down to 2.7 . Selfscreening, which reduces the PS to a value less than $\Delta$ (Snyder and Pask, 1973b), is also important in determining the reduction of the PS by the twist. The higher the absorption coefficient, the lower the PS (for a given $\Delta$ ) in an untwisted rhabdomere, and the less the reduction caused by the twist (see Fig. 8.13).

We conclude that, by twisting, a rhabdomere with a high dichroic ratio can have a low PS. The smaller the birefringence, the greater the reduction in the PS due to the twist. The absorption of unpolarised light as a function of twist angle is shown in Fig. 8.14. It can be seen from these curves that although twisting increases the absorption of unpolarised light for a given $\Delta$, the increase is small for reasonable values of $\Omega$, and certainly insufficient to increase the absorption of unpolarised light for $\Delta=10$ or 5 up to the same level as for $\Delta=2$. Only when the twist rate is very large are the two values approximately equal. The theoretical absorption for very fast rates of twist is given in Section 8.4 .2 as

$$
\mathrm{P}_{\mathrm{UP}}^{\mathrm{T}}=1-\mathrm{e}^{-\frac{\alpha \|^{\ell}}{2}}(1+1 / \Delta)
$$




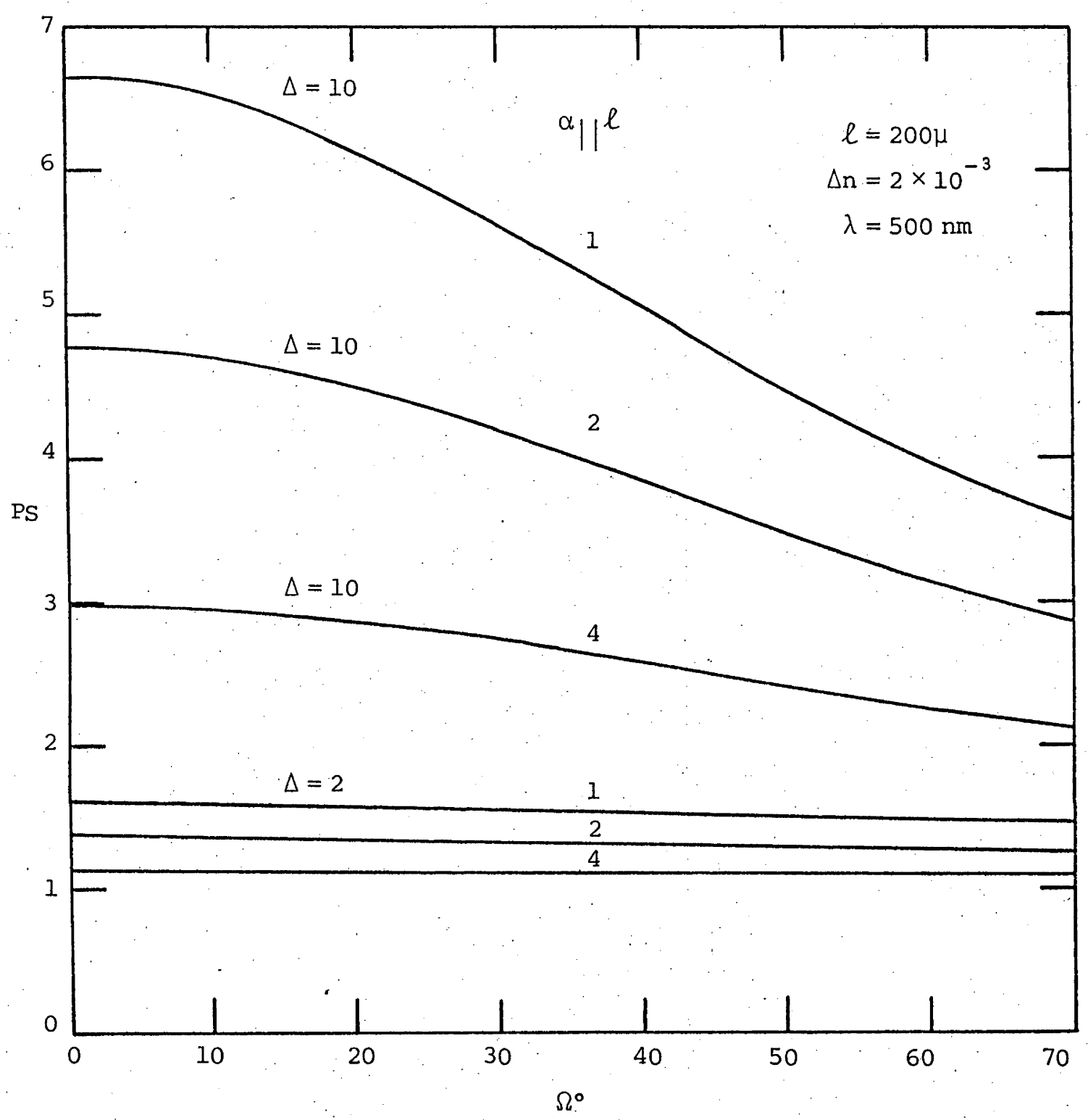

Fig. 8.13. The polarisation sensitivity of a fly-type rhabdomere as a function of total twist angle $\Omega$, with $\alpha l=1,2,4$ and $\Delta=2,10$. Waveguide effects are not included i.e. $n=1$. 


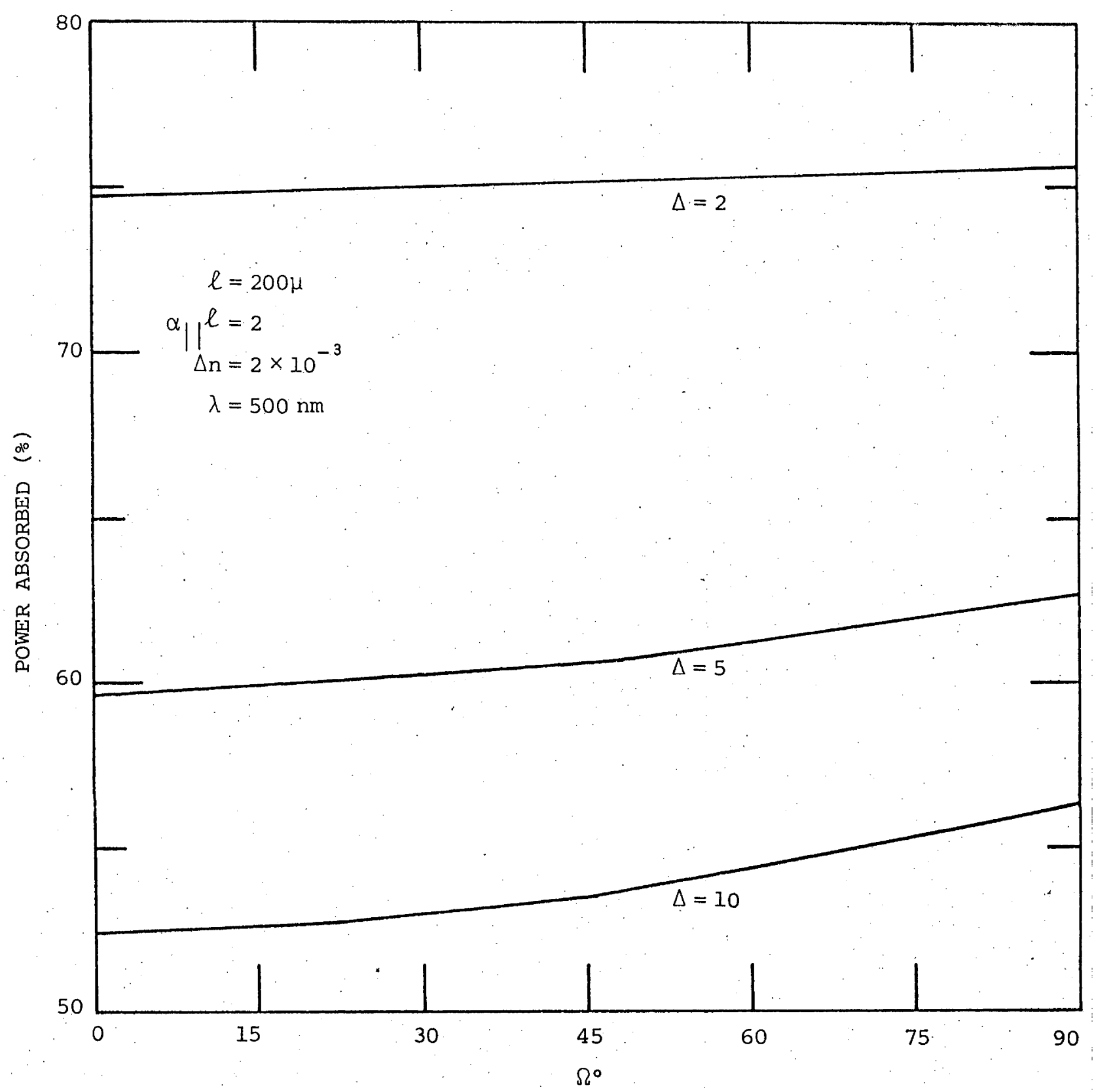

Fig. 8.14. Percent absorption of unpolarised light as a function of total twist angle $\Omega$. Table 8.3 gives the maximum values of the three curves. 
compared with the value (Snyder, 1973)

$$
\mathrm{P}_{\mathrm{UP}}^{\mathrm{NT}}=1-\frac{1}{2}\left(\mathrm{e}^{-\alpha} \|^{\ell}+\mathrm{e}^{-\frac{\alpha \|^{\ell}}{\Delta}}\right)
$$

when there is no twist. In Table 8.3 we give values for these two absorptions, using $\alpha_{\mid} l=2$.

\begin{tabular}{|r|l|l|}
\hline$\Delta$ & $\mathrm{P}_{\mathrm{UP}}^{\mathrm{NT}}(\%)$ & $\mathrm{P}_{\mathrm{UP}}^{\mathrm{T}}\left(\frac{\circ}{8}\right)$ \\
\hline 2 & 74.8 & 77.7 \\
5 & 59.7 & 69.9 \\
10 & 52.3 & 66.7 \\
\hline
\end{tabular}

Table 8.3

Figure 8.14 indicates that the total twist angle $\Omega$ must be very large for the absorption in a twisted rhabdomere to approach the minimum value $\mathrm{P}_{\mathrm{UP}}^{\mathrm{T}}$.

We conclude that the twisting of the rhabdomeres increases the absorption of unpolarised light, but that the absorption by twisted rhabdomeres with a high dichroic ratio is still significantly less than for rhabdomeres (twisted or untwisted) with $\Delta \approx 2$. On this basis we expect that $\Delta \approx 2$ in fly rhabdomeres, as predicted by snyder and Laughlin (1975).

Further evidence against the twisting of fly rhabdomeres is provided by Tauber (1974), who calculates the ellipticity and rotation of the polarisation ellipse for light passing along untwisted rhabdomeres. He shows that there is a good agreement with experimental data, which we would not expect if there was a significant twist.

On the other hand, indirect evidence for twisting is given by Horridge (1975). He finds that the angle for maximum absorption in the 
uv in fly rhabdomeres $1-6$ differs by about $60^{\circ}$ from that for maximum absorption in the green, and concludes that there are two absorbing pigments aligned at different angles; the most probable means of achieving this, according to Horridge, is for the pigments to lie at different depths in a twisted rhabdomere.

The arrangement of the central rhabdomere in the fly ommatidium is much the same in effect as the ninth cell in the ant or bee (Section 8.6.1). Rhabdomere 7 forms the distal half and has microvilli at right angles to those of rhabdomere 8, which lies underneath 7 (see Section 7.3). Therefore rhabdomere 7 can act as a polarisation filter for rhabdomere 8. Tauber (1974) shows that because these rhabdomeres are short and have orthogonal microvilli, light will remain approximately linearly polarised and the DP aligned with the microvilli. If the rhabdomeres are untwisted, they are therefore well suited for polarisation detection. However if the rhabdomeres are twisted, the discussion in section 8.6.1 on the ninth cell is relevant, the difference here being that fly rhabdomeres are birefringent and dichroic (c.f. the fused rhabdom). Therefore we would expect that the DP will rotate to some extent as the rhabdomeres twist, thereby maintaining the PS of rhabdomere 7 .

The effect of the twist on rhabdomere 8 is more difficult to quantify. If the incident light is polarised parallel or perpendicular to the microvilli of rhabdomere 7 and the DP follows the twist, unless the rotation of the DP is $90^{\circ}$, the DP will not be aligned for maximum or minimum absorption in rhabdomere 8 , therefore reducing its PS. If the incident light is polarised at an angle $\left(\neq 90^{\circ}\right)$ to the microvilli of rhabdomere 7 , the rotation of the DP and the ellipticity vary in a 
complicated fashion, therefore reducing the filtering effect of rhabdomere 7. The field at the distal end of rhabdomere 8 will in general be elliptically polarised and aligned at an angle to the microvilli, which will also reduce the PS of cell 8. A mitigating factor is that rhabdomeres 7 and 8 are short and therefore these effects may be small (T"auber 1974). The complicated manner in which the polarisation parameters vary in rhabdomeres 7 and 8 , if twisted, make it seem unlikely that they have evolved to make use of twisting.

\subsubsection{Birefringence of Twisted Rhabdomeres}

Kirschfeld and Snyder (1975) measure the birefringence of fly rhabdomeres and find a reduction in the birefringence with the length of the rhabdomere (their Fig. 10). They suggest that twisting may be responsible. In this section we use the theory of Section 8.5 to examine how the twist affects the measured birefringence.

We found in section 8.5 that the birefringence measured for a twisted medium is a complicated function of the actual birefringence and of the twist angle $\Omega=\xi \ell$, where $\xi$ is the rate of twisting. There are no optic axes in a twisted medium, so that the ellipticity and direction of polarisation of the field at the analyser vary periodically with $\Omega$; this gives rise to a periodic variation in the measured birefringence with length, rather than a simple reduction.

This is illustrated in Fig. 8.15, which shows the theoretical measured birefringence $\langle\Delta n\rangle$ as a function of length for several different rates of twist. This figure is given to show the general behaviour only, as it is not possible to include all parameters on a general curve and it is also difficult to account accurately for the results of Kirschfeld and Snyder (1975) without a more intimate knowledge 


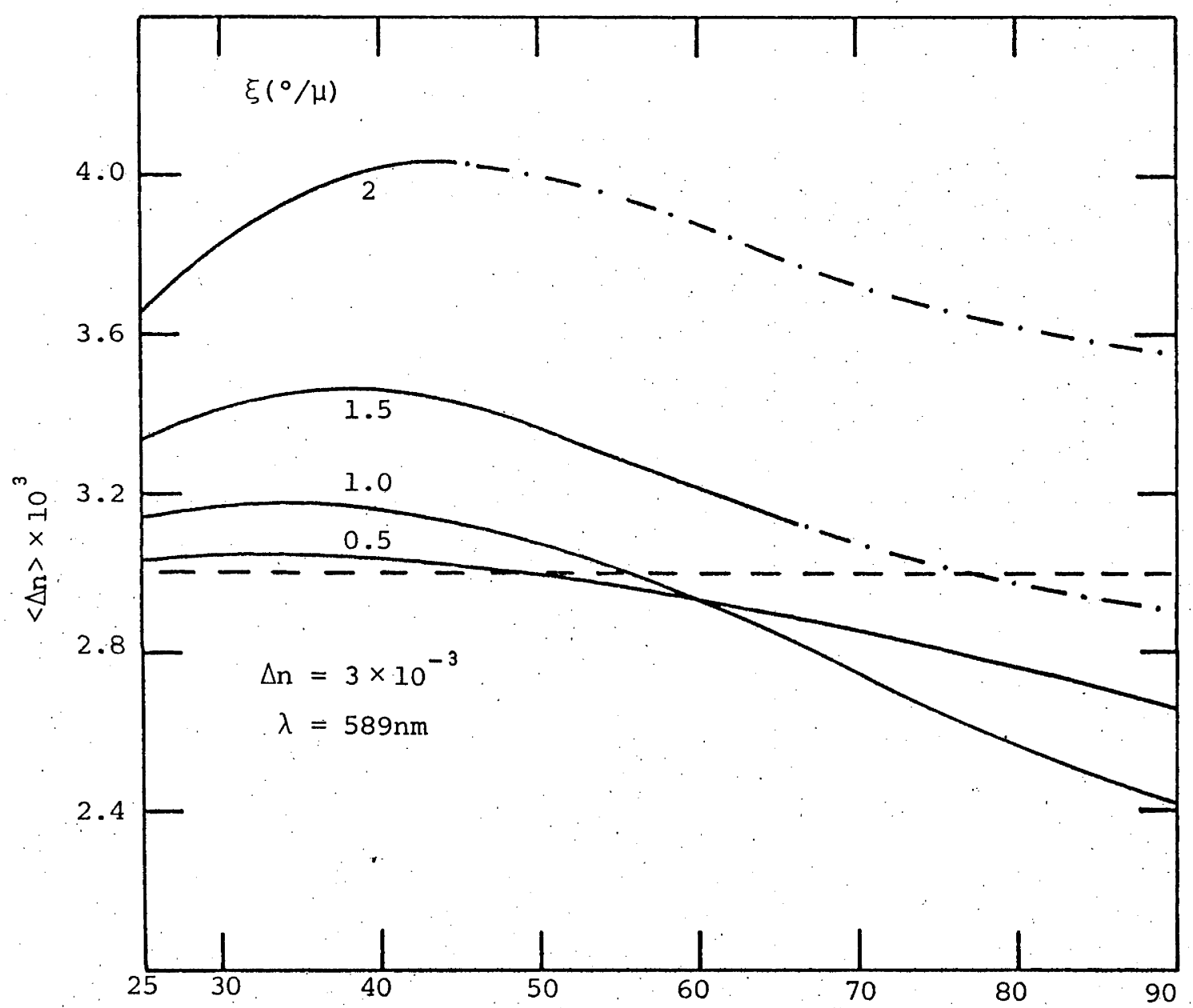

Fig. 8.15. An example of the effect of twisting on the measured birefringence $\langle\Delta n\rangle$. $\langle\Delta n\rangle$ is plotted as a function of length $l$ for a given rate of twist $\xi$. The dashed line indicates the untwisted value $\langle\Delta \mathrm{n}\rangle=3 \times 10^{-3}=\Delta \mathrm{n}$. The continuation -. - on the top two curves indicates the presence of the discontinuity (Section 8.5) which is ignored here. 
of the experiment. Figure 8.15 shows that as the twist rate decreases, the DP rotates with the twist and the measured birefringence approaches the true value $\Delta \mathrm{n}$. At faster rates of twist, the measured birefringence begins to vary rapidly with $\Omega$.

Difficulties in interpreting the experimental results are (1) the possibility that the twist is non-uniform over the length of the rhabdomere. The longer the rhabdomere, the more likely i.t is to be twisted;

(2) the inclusion of waveguide effects as indicated in Section 8.3. It is difficult to estimate values for the parameters if a significant proportion of the field propagates outside the twisted rhabdomere. A comparison of the length variation at different wavelengths may help to resolve this problem.

We conclude that there is no simple relationship between the actual and measured birefringence of a twisted rhabdomere, because there are no optic axes in a twisted medium. Figure 8.15 shows that over a suitable range of twist angles, the measured birefringence does decrease as found in the experiments of Kirschfeld and Snyder (1975), but that it can also increase. 


\section{REFERENCES}

Azzam, R.M.A. and Bashara, N.M. (1972), "Simplified approach to the propagation of polarized light in anisotropic media - application to Iiquid crystals", J. Opt. Soc. Am. 62, 1252.

Berreman, D.w. (1972), "Optics in stratified and anisotropic media: $4 \times 4$ matrix formulation", J. Opt. Soc. Am. 62, 502 .

Berreman, D.W. (1973), "Optics in smoothly varying planar structures: application to liquid-crystal twist cells", J. Opt. Soc. Am. $\underline{63}, 1374$.

Born, M. and Wolf, E. (1970), Principles of Optics (Pergamon Press, New York, 2nd edition).

Clarke, D. and Grainger, J.F. (1971), Polarized Light and Optical Measurement (Pergamon Press, Oxford).

De Vries, H.I. (1951), "Rotatory power and other optical properties of certain Iiquid crystals", Acta Cryst. $\underline{4}, 219$.

Gribakin, F.G. (1974), "Functional morphology of the compound eye of the bee", in Compound Eye and Vision of Insects, G.A. Horridge ed. (Clarendon Press, Oxford).

Gribakin, F.G. and Govardovskii, V.I. (1975), "The role of the photoreceptor membrane in photoreceptor optics", in Photoreceptor Optics, A.W. Snyder and R. Menzel eds. (Springer-Verlag, Heidelberg).

Grundler, O.J. (1974), "Elektronenmikroskopische Untersuchungen am Auge der Honigbiene (Apis mellifera)", Cytobiologie 9, 203.

Horridge, G.A. (1975), "Arthropod receptor optics", in Photoreceptor Optics, A.W. Snyder and R. Menzel eds. (Springer-Verlag, Heidelberg).

Hu, C. and Whinnery, J.R. (1974), "Losses of a nematic liquid-crystal optical waveguide", J. Opt. Soc. Am. 64, 1424.

Israelachvili, J., Sammut, R.A. and Snyder, A.W. (1975), "Birefringence and dichroism in invertebrate photoreceptors"., J. Opt. Soc. Am. 65, 221.

Kapany, N.S. (1967), Fiber Optics Principles and Applications (Academic Press, New York).

Kirschfeld, K. (1969), "Absorption properties of photopigments in single rods, cones and rhabdomeres", in Processing of Optical Data by Organisms and by Machines, w. Reichardt ed. (Academic Press, New York). 
Kirschfeld, K. and Snyder, A.W. (1975), "Waveguide mode effects, birefringence and dichroism in fly photoreceptors", in Photoreceptor Optics, A.W. Snyder and R. Menzel eds. (Springer-Verlag, Heidelberg).

Laughlin, S.B., Menzel, R. and Snyder, A.W. (1975), "Membranes, dichroism and receptor sensitivity", in Photoreceptor Optics, A.W. Snyder and R. Menzel eds. (Springer-Verlag, Heidelberg).

Louisell, W.H. (1960), Coupled Mode and Parametric Electronics (Wiley, New York) .

Marathay, A.S. (1971), "Matrix-operator description of the propagation of polarised light through cholesteric liquid crystals", J. Opt. Soc. Am. 61, 1363.

Marcuse, D. (1975), "Coupled-mode theory for anisotropic optical waveguides", B.S.T.J. $\underline{54}, 985$.

Mauguin, C. (1911), BuZZ. Soc. frane. Minêr 34, 71, quoted in De Vries (1951):

Menze1, R. (1972), "The fine structure of the compound eye of Formica polyctena - functional morphology of a Hymenopterean eye", in Information Processing in the Visual Systems of Arthropods, R. Wehner ed. (Springer-Verlag, Berlin):

Menzel, R. (1975), "Polarisation sensitivity in insect eyes with fused rhabdoms", in Photoreceptor Optics, A.W. Snyder and R. Menzel eds. (Springer-Verlag, Heidelberg).

Menzel, R. and Blakers, M. (1975), "Functional organisation of an insect ommatidium with fused rhabdom", submitted to Cytobiologie.

Menzel, R. and Snyder, A.W. (1974), "Polarised light detection in the bee, Apis mellifera", J. Comp. Physiol. 8.8, 247.

Schlecht, P. and Täuber, U. (1975), "The photochemical equilibrium in rhabdomeres of Eledone and its effect on dichroic absorption" in Photoreceptor Optics, A.W. Snyder and R. Menzel eds.. (Springer-Verlag, Heidelberg).

Shaw, S.R. (1969), "Interreceptor coupling in ommatidia of drone honey bee and locust compound eyes", Vision Res. 9, 999.

Shaw, S.R. (1969a), "Sense-cell structure and interspecies comparisons of polarized-light absorption in arthropod compound eyes", Vision Res. 9, 1031.

Snyder, A.W. (1973), "Polarisation sensitivity of individual retinula cells", J. Comp. Physiol. 83, 331.

Snyder, A.W. and Menzel, R. eds. (1975), Photoreceptor Optics (Springer-Verlag, Heidelberg). 
Snyder, A.W., Menzel, R. and Laughlin, S.B. (1973), "Structure and function of the fused rhabdom", J. Comp. Physiol. 87, 99.

Snyder, A.W. and Pask, C. (1973b), "Spectral sensitivity of Dipteran retinula cells", J. Comp. Physiol. 84, 59.

Snyder, A.W. and Sammut, R.A. (1973), "Direction of E for maximum response of a retinula cell", J. Comp. Physiol. 85, 37.

Stokes, A.R. (1963), The Theory of the Optical Properties of Intiomogeneous Materials (Spon, London).

Täuber, U. (1974), "Analyse des Polarisationszustandes des aus dem Rhabdomer austretenden Lichtes", J. Comp. Physiol. 95, 169. 


\section{BIBLIOGRAPHY}

Abramowitz, M. and Stegun, I.A. (1965), Hondbook of Mathematical Functions (Dover Publications, New York).

Adler, R.B. (1949), "Properties of guided waves on inhomogeneous cylindrical structures", M.I.T. Technical Report No. 102.

Arnaud, J.A. (1974), "Transverse coupling in fiber optics Part I: Coupling between trapped modes", B.S.T.J: $\underline{53}, 217$.

Arnaud, J.A. (1975), "Transverse coupling in fiber optics part IV: Crosstalk", B.S.T.J. 54, 1431.

Azzam, R.M.A. and Bashara, N.M. (1972), "Simplified approach to the propagation of polarized light in anisotropic media - application to liquid crystals", J. Opt. Soc. Am. 62, 1252.

Barlow, H.B. (1964), "Physical limits of visual discrimination", in Photophysiology, A.C. Giere ed., vol.. II (Academic Press, New York).

Bernard, G.D. (1975), "Physiological optics of the fused rhabdom", in Photoreceptor Optics, A.W. Snyder and R. Menzel eds. (SpringerVerlag, Heidelberg).

Berreman, D.W. (1972), "Optics in stratified and anisotropic media: $4 \times 4$ matrix formulation", J. Opt. Soc. Am. 62, 502.

Berreman, D.W. (1973), "Optics in smoothly varying planar structures: application to liquid-crystal twist cells", J. Opt. Soc. Am. $\underline{63}, 1374$.

Born, M. and Wolf, E. (1970), Principles of Optics (Pergamon Press, New York, 2nd edition).

Boschek, C.B. (1971), "On the fine structure of the peripheral retina and lamina ganglionaris of the fly, Musca domestica", Z. Zellforsch. $118,369$.

Bracey, M.F., Cullen, A.L., Gillespie, E.F.T. and Staniforth, J.A. (1959), "Surface wave research in Sheffield", I.R.E. Trans. Ant. Prop: AP-7, 5219.

Braitenberg, V. and Strausfeld, N.J. (1973), "Principles of the mosaic organisation in the visual system's neuropil of Musca domestica L", in Handbook of Sensory Physiology VII/3A, R. Jury ed. (Springerverlag, Berlin).

Brücke, E.w. von (1843), Archiv fur Anat. und Physiol. 11, 444 : quoted in O'Brien, B. (1946), "A theory of the Stiles and Crawford effect", J. Opt. Soc. Am. 36, 506. 
Burke, J.J. (1967), "Switching with fiber-optical waveguides", J. Opt. Soc. Am. 57, 1056.

Burkhardt, D. (1962), "Spectral sensitivity and other response characteristics of single visual cells in the arthropod eye", Symp. Soc. Exp. Biol. 16, 86 .

Burkhardt, D. (1964), "Colour discrimination in insects", Advane. Insect Physiol. 2, 131.

Cherin, A.H. and Murphy, D.J. (1975), "Quasi-ray analysis of crosstalk between multimode' optical fibers", B.S.T.J. 54, 17.

Clarke, D. and Grainger, J.F. (1971), Polarized Light and Optical Measurement (Pergamon Press, Oxford).

Clarricoats, P.J.B. (1975), "Theory of optical fibre waveguides a review", in Progress in Optics, E. Wolf ed. (North Holland Publishing Co., Amsterdam).

Clarricoats, P.J.B. and Chan, K.B. (1973), "Propagation behaviour of cylindrical-dielectric-rod waveguides", PrOC. I.E.E. 120, 1371.

Collin, R.E. (1961), Field Theory of Guided Waves (McGraw-Hill, New York):

Dartnall, H.J.A. ed. (1972), Handbook of Sensory Physiology VII/1: Photochemistry of Vision (Springer-Verlag, Berlin).

De Vries, H.L. (1951), "Rotatory power and other optical properties of certain liquid crystals", Acta Cryst. $\underline{4}, 219$.

Dörrscheidt-Käfer, M. (1972), "Die Empfindlichkeit einzelner Photorezeptoren in Komplexauge von Calliphora erthrocephala", J. Comp. Physiol. 81, 309.

Enoch, J.M. (1963), "Optical properties of retinal receptors", J. Opt. Soc. Am. 53, 71 .

Enoch, J.M. (1967), "Comments on Excitation of waveguide modes in retinal receptors", J. Opt. Soc. Am. 57, 548 .

Franceschini, N. and Kirschfeld, K. (1971), "Etude optique in vivo des éléments photorecepteurs dans l!oeil composé de Drosophila", Kybernetik $81,1$.

Friedman, B. (1956), Principles of Applied Mathematics (Wiley, New York).

Fuortes, M.G.F. ed. (1972), Handbook of Sensory Physiology VII/2: Physiology of Photoreceptor Organs (Springer-Verlag, Berlin).

Gloge, D. (1971), "Weakly guiding fibers", Appl. Opt. 10, 2252.

Gloge, D. (1972), "Bending loss in multimode fibers with graded and ungraded core index", Appl. Opt. 11, 2506. 
Gloge, D. (1974), "Optical fibers for communication", Appl. Optics 13,249 .

Gloge, D. and Marcatili, E.A.J. (1973), "Multimode theory of gradedcore fibers", B.S.T.J. 52, 1563.

Goldsmith, T.N. and Bernard, G.D. (1974), "The visual system of insects", in Physiology of Insecta, vol. II, M. Rockstein ed. (Academic Press, New York).

Goodman, J.W. (1968), Introduction to Fourier Optics (McGraw-Hill, San Francisco).

Gribakin, F.G. (1974), "Functional morphology of the compound eye of the bee", in Compound Eye and Vision of Insects, G.A. Horridge ed. (Clarendon Press, Oxford).

Gribakin, F.G. and Govardovskii, V.I. (1975), "The role of the photoreceptor membrane in photoreceptor optics", in Photoreceptor Optics, A.W. Snyder and R. Menzel eds. (Springer-Verlag, Heidelberg).

Grundler, O.J. (1974), "Elektronenmikroskopische Untersuchungen am Auge der Honigbiene (Apis mellifera)", Cytobiologie 9, 203.

Hamdorf, K. and Schwemer, J. (1975), "Photoregeneration and the adaptation process in insect photoreceptors", in Photoreceptor Optics, A.W. Snyder and R. Menzel eds. (Springer-Verlag, Heidelberg).

Horridge, G.A. ed. (1974), The Compound Eye and Vision of Insects (Clarendon Press, Oxford).

Horridge, G.A. (1975), "Arthropod receptor optics", in Photoreceptor Optics, A.W. Snyder and R. Menzel eds. (Springer-Verlag, Heidelberg).

Hu, C. and Whinnery, J.R. (1974), "Losses of a nematic liquid-crystal optical waveguide", J. Opt. Soc. Am. 64, 1424.

Israelachvili, J., Sammut, R.A. and snyder, A.W. (1975), "Birefringence and dichroism in invertebrate photoreceptors", J. Opt. Soc. Am. 65, 221.

Jackson, J.D. (1962), Classical Electrodynomics (Wiley, New York).

Johnson, C.C. (1965), Field and Wave Electrodynamics (McGraw-Hill, New York).

Jones, A.L. (1965), "Coupling of optical fibers and scattering in. fibers", J. Opt. Soc. Am. 55, 261.

Kapany, N.S. (1959), "Fiber optics. V. Light leakage due to frustrated total reflection", J. Opt. Soc. Am. $\underline{49}, 770$. 
Kapany, N.S. (1959a), "Fiber optics. VI. Image quality and optical insulation", J. Opt. Soc. Am. 49, 779.

Kapany, N.S. (1967), Fiber Optics Principles and Applications (Academic Press, New York).

Kapany, N.S., Burgwald, G.M. and Burke, J.J. (1965), "Light amplification and switching using fiber optics and laser", in optical and Electro-optical Information Processing, J.T. Tippett et al (eds.) (M.I.T. Press, Cambridge), Ch.17.

Kapany, N.S. and Burke, J.J. (1972), Optical Waveguides (Academic Press, New York).

Kirschfeld, K. (1969), "Absorption properties of photopigments in single rods, cones and rhabdomeres", in Processing of optical Data by organisms and by Machines, w. Reichardt ed. (Academic Press, New York).

Kirschfeld, K. (1969a), "Optics of the compound eye", in Processing of Optical Data by Organisms and by Machines, w. Reichardt ed. (Academic Press, New York).

Kirschfeld, K. (1972), "The visual system of Musca: Studies on optics, structure and function", in Information Processing in the Visual System of Arthropods, R. Wehner ed. (Springer-Verlag, Berlin).

Kirschfeld, K. (1975); "The compound eye of the fly: Differences and analogies to vision in vertebrates", submitted to Science.

Kirschfeld, K. and Franceschini, N. (1969), "Ein Mechanismus zur Steurung des Lichtflusses in den Rhabdomeren des Komplexauges von Musca", Kybernetik $6,13$.

Kirschfeld, K. and Snyder, A.W. (1975), "Waveguide mode effects, birefringence and dichroism in fly photoreceptors", in Photoreceptor Optics, A.W. Snyder and R. Menzel eds. (SpringerVerlag, Heidelberg).

Kuiper, J.W. (1966), "On the image formation in a single ommatidium of the compound eye in Diptera", in The Functional Organisation of the Compound Eye, C.G. Bernhard ed. (Pergamon Press, Oxford).

Kuwahara, H., Hamasaki, J. and Saito, S. (1975), "Power transfer of a parallel optical fiber directional coupler", I.E.E.E. Trans. Microwave Theory Tech. MTT-23, 178.

Laughlin, S.B., Menzel, R. and Snyder, A.w. (1975), "Membranes, dichroism and receptor sensitivity", in Photoreceptor Optics, A.W. Snyder and R. Menzel eds. (Springer-Verlag, Heidelberg).

Liebman, P.A. and Entine, G. (1968), "Visual pigments of frog and tadpole", Vision Res. $\underline{8}, 761$.

Louisell, W.H. (1955), "Analysis of the single tapered mode coupler", B.S.T.J. 34,853 . 
Louisell, W.H. (1960), Coupled Mode and Parcmetric Electronics (Wiley, New York).

Marathay, A.S. (1971), "Matrix-operator description of the propagation of polarised light through cholesteric liquid crystals", J. Opt. Soc. Am. 61, 1363.

Marcatili, E.A.J. (1969), "Dielectric rectangular waveguide and directional coupler for integrated optics", B.S.T.J. 48,2071 .

Marcuse, D. (1971), "The coupling of degenerate modes in two parallel dielectric waveguides", B.S.T.J. 50, 1791.

Marcuse, D. (197la), "Bending losses of the asymmetric slab waveguide", B.S.T.J. 50, 2551.

Marcuse, D. (1972), Light Transmission Optics (Van Nostrand Press, New York).

Marcuse, D. (1973), "Optical fibers for communications", Radio Elec. Engineer 43, 655.

Marcuse, D. (1973a), "Coupled mode theory of round optical fibers", B.S.T.J. 52,817 .

Marcuse, D. (1974), Theory of Dielectric Optical Waveguides (Academic Press, New York).

Marcuse, D. (1975), "Coupled-mode theory for anisotropic optical waveguides", B.S.T.J. 54, 985.

Matsuhara, M. and Kumagai, N. (1974), "Theory of coupled open transmission lines and its applications", I.E.E.E. Microwave Theory Tech. MTT-22, 378.

Matsuhara, M. and Watanabe,"A. (1975), "Coupling of curved transmission lines and application to optical directional couplers", J. Opt. Soc. Am. 65, 163.

Mauguin, C. (1911), Buzl. Soc. franc. Minér 34, 71, quoted in De Vries (1951).

Maurer, R.D. (1973), "Glass fibers for optical communications", Proc. I.E.E.E. 61, 452 .

Melamed, J. and Trujillo-Cenóz, O. (1968), "The fine structure of the central cells in the ommatidia of Dipterans",

J. UZtrastruct. Res. 21, 313 .

Menzel, R. (1972), "The fine structure of the compound eye of Formica polyctena - functional morphology of a Hymenopterean eye", in Information Processing in the Visual Systems of Arthropods, R. Wehner ed. (Springer-Verlag, Berlin). 
Menzel, R. (1975), "Polarisation sensitivity in insect eyes with fused rhabdoms", in Photoreceptor Optics, A.W. Snyder and R. Menzel eds. (Springer-Verlag, Heidelberg).

Menzel, R. and Blakers, M. (1975), "Functional organisation of an insect ommatidium with fused rhabdom", submitted to Cytobiologie.

Menzel, R. and Snyder, A.W. (1974), "Polarised light detection in the bee, Apis mellifera", J. Comp. Physiol. 88, 247.

Midwinter, J. (1975), "The prism-taper coupler for the excitation of single modes in optical transmission fibres", Optical and Quantum Elect. 7. 297.

Miller, S.E. (1954), "Coupled wave theory and waveguide applications", B.S.T.J. 33,661 .

Miller, S.E., Marcatili, E.A.J. and Tingye Li (1973), "Research toward optical-fiber transmission systems", Proc. I.E.E.E. 61, 1703.

Miller, W.H. and Snyder, A.W. (1973), "Optical function of human peripheral cones", Vision Res. 13, 2185.

Miller, W.H. and Snyder, A.W. (1976), "Resolving power of eagles and hawks", in preparation.

Moon, P.H. and Spencer, D.E. (1971), Field Theory Handbook (Springer-Verlag, Berlin).

Pask, C. and Snyder, A.W. (1975), "Theory of the Stiles-Crawford effect of the second kind", in Photoreceptor. Optics, A.W. Snyder and

R. Menzel eds. (Springer-Verlag, Heidelberg).

Pask, C. and Snyder, A.W. (1975a), "Angular sensitivity of lensphotoreceptor systems", in Photoreceptor Optics, A.W. Snyder and R. Menzel eds. (Springer-Verlag, Heidelberg).

Pask, C., Snyder, A.W. and Mitchell, D.J. (1975), "Number of modes on optical waveguides", J. Opt. Soc. Am. $\underline{65}, 356$.

Pierce, J.L. (1954)., "Coupling of modes of propagation", J. Appl. Phys. 25, 179.

Reichardt, w. ed. (1969), Processing of Optical Data by Organisms and by Machines (Academic Press, New York).

Reisinger, A. (1973), "Characteristics of optical guided modes in lossy waveguides", Appl. Opt. 12, 1015.

Rowe, H.E. (1962), "Approximate solution for the coupled line equations", B.S.T.J. 41 , 1011 .

Sammut, R.A. (1975), "Theory of unbound modes on circular dielectric waveguides", Ph.D. Thesis, Australian National University, Canberra. 
Sammut, R.A., Pask, C. and Snyder, A.W. (1975), "Excitation and power of the unbound modes within a circular dielectric waveguide", Proc. I.E.E.E. 122, 25.

Sammut, R.A. and Snyder, A.W. (1974), "Contribution of unbound modes to light absorption in visual photoreceptors", J. Opt. Soc. Am. 64, 1171.

Sandbank, C.P. (1975), "Fibre optic communications: a survey", Elec. Commun. 50, 20.

Schelkunoff, S.A. (1955), "Conversion of Maxwell's equations into generalized telegraphist's equations", B.S.T.J. 34, 995.

Schlecht, P. and Täuber, U. (1975), "The photochemical ëquilibrium in rhabdomeres of Eledone and its effect on dichroic absorption" in Photoreceptor Optics, A.W. Snyder and R. Menzel eds. (Springer-Verlag, Heidelberg).

Seitz, G. (1968), "Der Strahlengang im Appositionsauge von Calliphora erythrocephala (Meig.)", Z. Vergl. Physiol. 59, 205.

Shaw, S.R. (1969), "Interreceptor coupling in ommatidia of drone honey bee and locust compound eyes", Vision Res. 9, 999.

Shaw, S.R. (1969a), "Sense-cell structure and interspecies comparisons of polarized-light absorption in arthropod compound eyes", Vision Res. 9, 1031.

Smith, R.B. (1975), "Coupling efficiency of the tapered coupler", Electron. Lett. 11, 204.

Snitzer, E. (1961), in Advances in Quantum Electronics, J.R. Singer, ed. (Columbia University Press, New York).

Snitzer, E. (1963), in Optical Processing of Information, D.K. Pollock et al, eds. (Spartan Books, Baltimore).

Snyder, A.W. (1969), "Wave propagation along dielectric structures with application to retinal receptors", Ph.D. Thesis, University of London.

Snyder, A.W. (1969a), "Asymptotic expressions for eigenfunctions and eigenvalues of a dielectric or optical waveguide", I.E.E.E. Trans. Microwave Theory Tech. MTT-17, 1130.

Snyder, A.W. (1969b), "Excitation and scattering of modes on a dielectric or optical fiber", I.E.E.E. Trans. Microwave Theory Tech: MTT-17, 1138.

Snyder, A.W. (1970), "Coupling of modes on a tapered dielectric cylinder", I.E.E.E.Trans. Microwave Theory Tech. MTT-18, 383.

Snyder, A.W. (1971), "Continuous mode spectrum of a circular dielectric rod", I.E.E.E. Microwave Theory Tech. MTT-19, 720. 
Snyder, A.w. (1971a), "Approximate eigenvalues for a circular rod of arbitrary relative permittivity", Electron. Lèts. 7. 105 .

Snyder, A.w. (1971b), "Mode propagation in a nonuniform cylindrical medium", I.E.E.E. Trans. Microwave Theory Tech. MTT-19, 402.

Snyder, A.W. (19.72), "Coupled-mode theory for optical fibers", J. Opt. Soc. Am. 62, 1267.

Snyder, A.W. (1972a), "Power loss on optical fibers", PrOC. I.E.E.E. 60, 757.

Snyder, A.w. (1973), "Polarisation sensitivity of individual retinula cells", J. Comp. Physiol. 83, 331.

Snyder, A.W. (1974), "Leaky-ray theory of optical waveguides of circular cross section", Appl. Phys. 4,273 .

Snyder, A.W. (1974a), "Light absorption in visual photoreceptors", J. Opt. Soc. Am. 64, 216.

Snyder, A.W. (1974b), "Optical properties of invertebrate photoreceptors", in The Compound Eye and Vision of Insects, G.A. Horridge ed. (Clarendon Press, Oxford).

Snyder, A.W. (1975), "Photoreceptor optics - theoretical principles", in Photoreceptor Optics, A.W. Snyder and R. Menzel eds. (Springer-Verlag, Heidelberg).

Snyder, A.W: (1976), "Acuity of compound eyes", submitted to J. Comp. Physiol.

Snyder, A.W. and Horridge, G.A. (1972); "The optical function of changes in the medium surrounding the cockroach rhabdom",

J. Comp. Physiol. 81,1 .

Snyder, A.W. and Love, J.D. (1975), "Reflection at a curved dielectric interface - electromagnetic tunnelling", I.E.E.E. Trans. Microwave Theory Tech. MTT-23, 134.

Snyder; A.W. and Menzel, R. eds. (1975), Photoreceptor Optics (Springer-Verlag, Heidelberg).

Snyder, A.W., Menzel, R. and Laughlin, S.B. (1973), "Structure and function of the fused rhabdom", J. Comp. Physiol. 87, 99.

Snyder, A.W. and Miller, W.H. (1972), "Fly colour vision", Vision Res. 12, 1389.

Snyder, A.W. and Mitchell, D.J. (1974), "Leaky rays on circular optical fibers", J. Opt. Soc. Am. 64, 599.

Snyder, A.W. and Pask, C. (1972), "Light absorption in the bee photoreceptor", J. Opt. Soc. Am. 62, 998.

Snyder, A.W. and Pask, C. (19.72a), "Angular sensitivity of the bee ommatidium", J. Comp. Physiol. 76, 438 . 
Snyder, A.W. and Pask, C. (1972b), "A theory for changes in spectral sensitivity induced by off-axis light", J. Comp. Physiol. $\underline{79}, 423$.

Snyder, A.W. and Pask, C. (1973), "Incoherent illumination of an optical fiber", J. Opt. Soc. Am. 63, 806.

Snyder, A.W. and Pask, C. (1973a), "Absorption in conical optical fibers", J. Opt. Soc. Am. 6.3, 761.

Snyder, A.W. and Pask, C. (1973b), "Spectral sensitivity of Dipteran retinula cells", J. Comp. Physiol. 84, 59.

Snyder, A.W. and Pask, C. (1973c), "The Stiles-Crawford effect explanation and consequences", Vision Res. 13, 1115.

Snyder, A.W. and Pask, C. (1975), "Optical fibre: Spatial transient and steady state", Opt. Comm. 15, 314.

Snyder, A.W., Pask, C. and Mitchell, D.J. (1973), "Light-acceptance property of an optical fiber", J. Opt. Soc. Am. 63, 59.

Snyder, A.W. and Sammut, R.A. (1973), "Direction of E for maximum response of a retinula cell", J. Comp. Physiol. 85, 37.

Stavenga, D.G. (1974), "Visual receptor optics, rhodopsin and pupil in fly retinula cells", Thesis, Groningen.

Stavenga, D.G. (1975), "Optical qualities of the fly eye - an approach from the side of geometrical, physical and waveguide optics", in Photoreceptor Optics, A.W. Snyder and R. Menzel eds. (Springer-Verlag, Heidelberg).

Stavenga, D.G., Zantema, A. and Kuiper, J.W. (1973), "Rhodopsin processes and the function of the pupil mechanism in flies", in Biochemistry and Physiology of Visual Pigments, H. Langer ed. (Springer-Verlag, Berlin).

Stokes, A.R. (1963), The Theory of the Optical Properties of Inhomogeneous Materials (Spon, London).

Stratton, J.A. (1941), Electromagnetic Theory (McGraw-Hill, New York).

Täuber, U. (1974), "Analyse des Polarisationszustandes des aus dem. Rhabdomer austretenden Lichtes", J. Comp. Physiol. 95, 169.

Taylor, H.F. and Yariv, A. (1974), "Guided wave optics", Proc. I.E.E.E. 62, 1044.

Toraldo di Francia, G. (1948), "Per una teoria dell' effeto StilesCrawford", Il Nuovo Cimento $\underline{5}, 589$.

Trujillo-Cenóz, 0. (1972), "The structural organisation of the compound eye in insects", in Handbook of Sensory Physiology VII/2,

M.G.F. Fuortes ed. (Springer-Verlag, Berlin). 
Trujillo-Cenóz, O. and Bernard, G.D. (1972), "Some aspects of the retinal organization of Sympycnus Zineatus Loew (Diptera, Dolichopodidae)", J. Ultrastruct. Res. $38,149$.

Trujillo-Cenóz, O. and Melamed, J. (1966), "Electron microscope observations on the peripheral and intermediate retinas of Dipterans", in The Functional Organisation of the Compound Eye, C.G. Bernhard ed. (Pergamon Press, Oxford).

Vanclooster, R. and Phariseau, P. (1970), "The coupling of two parallel dielectric fibers I. Basic equations", Physica 47, 485.

Vanclooster, R. and Phariseau, P. (1970a), "The coupling of two parallel dielectric fibers II. Characteristics of the coupling in two fibers", Physica 47, 501.

Vanclooster, R. and Phariseau, P: (1970b), "Diffraction of an electromagnetic wave by a fiber bundle", Physica $\underline{50}, 308$.

Varela, F.G. and Wiitanen, W. (1970), "The optics of the compound eye of the honeybee (Apis mellifera)", J. Gen. Physiol. 55,336 .

Walls, G. (1972), The Vertebrate Eye (Cranbrook Press, Michigan).

Wehner, R. ed. (197.2), Information Processing in the Visual System of Arthropods (Springer-Verlag, Berlin).

Wijngaard, W. (1971), "Depolarization of plane-polarized light by light-guiding frog rods", J. Opt. Soc. Am. 61, 1187.

Wijngaard, W. (1973), "Guided normal modes of two parallel circular dielectric rods", J. Opt. Soc. Am. 63, 944.

Wijngaard, W. (1974), "Some normal modes of an infinite hexagonal array of identical circular dielectric rods", J. Opt. Soc. Am. 64, 1136.

Wijngaard, W. and Heyker, H. (1975), "Optical interaction between retinal receptors", in Photoreceptor Optics, A.W. Snyder and R. Menzel eds. (Springer-Verlag, Heidelberg).

Wijngaard, W. and Stavenga, D.G. (1975), "On optical crosstalk between fly rhabdomeres", Biol. Cybernetics 18, 61 .

Wilson, M.G.F. and Teh, G.A. (1973), "Improved tolerance in optical directional couplers", Electron. Lett. $\underline{9}, 453$.

Wilson, M.G.F. and Teh, G.A. (1975), "Tapered optical directional coupler"., I.E.E.E. Microwave Theory Tech. MTT-23, 85.

Yeh, C. (1972), "Noncircular dielectric waveguides", in Optical Waveguides, N.S. Kapany and J.J. Burke (Academic Press, New York), Appendix A. 